\title{
A systematic study of variability among OB-stars based on HIPPARCOS photometry
}

\author{
L. Lefèvre ${ }^{1,2}$, S. V. Marchenko ${ }^{3}$, A. F. J. Moffat ${ }^{2}$, and A. Acker ${ }^{4}$ \\ 1 LESIA, CNRS UMR 8109, Observatoire de Paris, Université Paris 6, Université Paris 7, 92195 Meudon Cedex, France \\ e-mail: laure.lefevre@obspm.fr \\ 2 Département de Physique, Université de Montréal, Observatoire du Mont Mégantic, Centre de Recherche en Astrophysique \\ du Québec, Montréal, QC H3C 3J7, Canada \\ e-mail: [lefevre,moffat] aastro.umontreal.ca \\ 3 Department of Physics and Astronomy, WKU, Bowling Green, KY 42101-3576, USA \\ e-mail: sergey@astro.wku.edu \\ ${ }^{4}$ Observatoire de Strasbourg, 11 rue de l'Université, 67000 Strasbourg, France \\ e-mail: acker@astro.u-strasbg.fr
}

Received 8 April 2009 / Accepted 6 August 2009

\begin{abstract}
Context. Variability is a key factor for understanding the nature of the most massive stars, the OB stars. Such stars lie closest to the unstable upper limit of star formation.

Aims. In terms of statistics, the data from the HIPPARCOS satellite are unique because of time coverage and uniformity. They are ideal to study variability in this large, uniform sample of OB stars.

Methods. We used statistical techniques to determine an independant threshold of variability corresponding to our sample of OB stars, and then applied an automatic algorithm to search for periods in the data of stars that are located above this threshold. We separated the sample stars into 4 main categories of variability: 3 intrinsic and 1 extrinsic. The intrinsic categories are: OB main sequence stars ( $\sim 2 / 3$ of the sample), OBe stars $(\sim 10 \%)$ and OB Supergiant stars $(\sim 1 / 4)$. The extrinsic category refers to eclipsing binaries.

Results. We classified about $30 \%$ of the whole sample as variable, although the fraction depends on magnitude level due to instrumental limitations. OBe stars tend to be much more variable $(\approx 80 \%)$ than the average sample star, while OBMS stars are below average and OBSG stars are average. Types of variables include $\alpha \mathrm{Cyg}, \beta \mathrm{Cep}$, slowly pulsating stars and other types from the general catalog of variable stars. As for eclipsing binaries, there are relatively more contact than detached systems among the OBMS and OBe stars, and about equal numbers among OBSG stars.
\end{abstract}

Key words. methods: statistical - methods: data analysis - techniques: photometric - catalogs - stars: variables: general stars: fundamental parameters

\section{Introduction}

OB stars are taken to comprise O to B3 stars of all luminosity classes and B4 to B8 supergiants as explained in the atlas of Walborn \& Fitzpatrick (1990). In fact, OB does not refer to the whole range of $\mathrm{O}$ and $\mathrm{B}$ stars but only to the most massive $\mathrm{O}$ and B stars that usually core-collapse as type II, Ib or Ic supernovae, depending on their initial mass. They also define the arms in spiral galaxies. O stars in particular are precursors of Wolf-Rayet stars, which considerably stir up and enrich their immediate environments in heavy elements in the process.

The most massive stars tend to be the most unstable (close to the Eddington limit) and, like WR stars, sometimes have strong, optically-thick stellar winds that prevent us from seeing their hydrostatic surface and thus deducing their characteristics directly. Thus, studies of their photometric variations can be more revealing in an indirect way. First, the presence of a periodic phenomenon in a given star enables one to constrain its parameters. If the variability is intrinsic, i.e. from within the star itself, it allows one to probe the otherwise mostly inaccessible internal parameters of a massive star. Second, when studying a whole sample of stars, classification of different types of variability helps to deduce statistical properties of a subclass of massive stars. Depending on the period and/or amplitude of the phenomenon, one can deduce a most likely responsible mechanism and judge if such a mechanism dominates in a given subcategory of stars.

The HIPPARCOS (ESA 1997, The Hipparcos and Tycho catalogs, ESA-SP1200) satellite offers a unique opportunity to study an unbiased sample of OB stars in exactly this manner. It provides a homogeneous set of photometric data for a large sample of OB stars, over a relatively long period of time ( $\sim 3$ years). Moreover, a period search of the variable candidates has already been undertaken by the HIPPARCOS consortium, thus providing us with an initial basis of our study. We exploit here the HIPPARCOS photometric results concerning OB stars by reanalyzing them in a more accurate and systematic way.

In Sect. 2 we present the selection of targets. Section 3 details the method used for the detection of variable stars in this sample. In Sect. 4, we briefly explain the analysis of the periodicities in the sample stars and in Sect. 5 we present and discuss the different results beginning with the extrinsic variability, i.e. due to effects outside the star, then proceeding to the different classes of intrinsic variability. Section 6 concludes our analysis. 


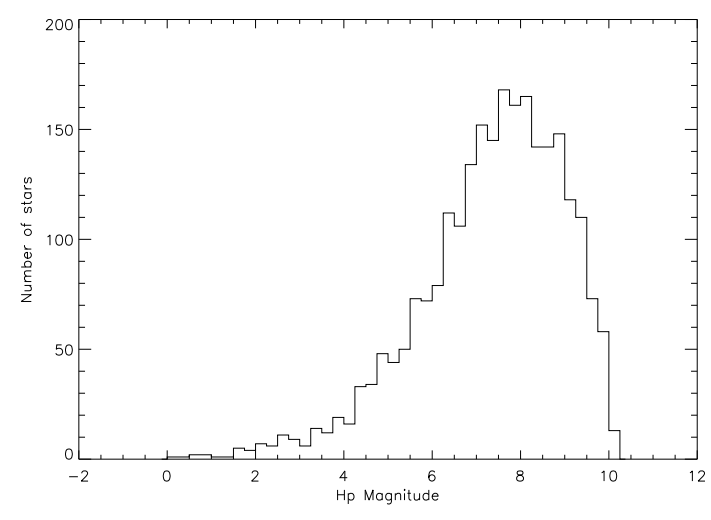

Fig. 1. Histogram of the magnitude of the stars in our sample. The cutoff near $H p=10$ mag is completely artificial as it corresponds to the limit we chose for our sample. Note the steep decrease of the histogram above $H p=8 \mathrm{mag}$, where the sample is not complete.

\section{Selection of OB stars}

We selected OB stars from the VIZIER database and created a photometric catalog of the selected targets from the HIPPARCOS CD-rom. We selected only stars with magnitude down to $H p=10$ mag because of the rapid growth of the instrumental error below that limit.

Although it is now evident from Fig. 1 that the sample is only complete down to $H p \sim 8$, we examine the whole sample of 2497 stars (including 13 stars for which new calculations are slightly fainter than $\mathrm{Hp}=10$ and have thus been omitted from further study). Excluded from this study are Wolf-Rayet stars (studied by Marchenko et al. 1998) and most Luminous Blue Variables (LBVs). Note that 3 confirmed LBVs (namely HR Car, AG Car and P Cyg) are included in the OBe category as their extracted Vizier spectral types do not reflect their supergiant status (B2evar, B2:pe and B2pe, respectively). The small influence they could have on the variability levels of this class will be discussed in Sect. 5.3.

Each data point for each star is flagged with a 9-bit number, indicating if any problem was encountered during the exposure. Each bit corresponds to a precise problem, and the resulting flag is a combination of bits. Rather than boldly rejecting all flagged points, we have chosen the most "damaging" flags by carefully assessing the influence of the flagged points on the light curves of non variable stars, in an attempt to minimize both the number of rejected points and the number of rejected stars. The most damaging bits were selected (mainly sun pointing, high background and object in either field of view) and we removed the points corresponding to the flags. These flags are represented by these bits exactly or any combination of them.

Each point also is assigned an instrumental error based primarily on Poisson statistics. Some of the points with a larger error do not have significantly "bad" flags, while some points with "bad" flags do not present a larger error. Thus we decided to adopt a method which takes into account flags and measurement errors. First, we eliminated the problematic data-points for each star according to their flags. Typically 3 points per star on average were flagged and thus deleted. A total of 77 stars were excluded from the analysis (including 7 previously known periodically varying stars) because $>10 \%$ of their points were flagged as problematic. The inclusion of measurement errors is explained in Sect. 3. Note that the variability characteristics of these 7 previously known periodically varying stars will be taken into account in the final statistics.

\section{Detection of variables}

Each HIPPARCOS star was assigned by the HIPPARCOS consortium a "variability flag", which indicates whether it is variable (periodic "P", unsolved variable "U", or microvariable "M"), constant ("C"), or presented problems during the reduction (mainly Duplicity-induced variable, i.e. instrumental, "D", or Revised-color-index "R"). Some of the stars could not be entered into any of the above categories (not-classified " ", i.e. no flag). We computed a weighted mean and amplitude for each of these stars to correctly allow for variable quality. In a last step (a posteriori) we also deleted a few points from a limited number of targets, after checking their lightcurves by eye, i.e. points more than approximately $3 \sigma$ from the mean. The following formulae were used to calculate the weighted mean and amplitude for each star, where "peak-to-peak amplitude" $=3.289 * S$ (ESA 1997, The Hipparcos and Tycho catalogs, Vol. 1, Eq. (1.3.14)).

$$
\begin{aligned}
\mu_{w} & =\frac{\sum_{i=1}^{N}\left(\frac{X_{i}}{\sigma_{i}^{2}}\right)}{\sum_{i=1}^{N} \frac{1}{\sigma_{i}^{2}}} \approx \mu=\frac{\sum_{i=1}^{N} X_{i}}{N}, \text { for } \sigma_{i} \approx \mathrm{cst} . \\
\sigma_{w}^{2} & =\frac{\sum_{i=1}^{N} \frac{\left(X_{i}-\mu\right)^{2}}{\sigma_{i}^{2}}}{\sum_{i=1}^{N} \frac{1}{\sigma_{i}^{2}}} \approx \sigma^{2}=\frac{\sum_{i=1}^{N}\left(X_{i}-\mu\right)^{2}}{N}, \text { for } \sigma_{i} \approx \mathrm{cst} . \\
S^{2} & =\frac{\sum_{i=1}^{N}\left(X_{i}-\mu\right)^{2}}{N-1} \approx \sigma^{2} \times \frac{N}{N-1}, \text { for } \mathrm{N} \gg 1 .
\end{aligned}
$$

$X_{i}$ is the individual observation for a star, and $\sigma_{i}$ is the error on this individual observation. As can be seen, $\mu_{w}$ and $\sigma_{w}^{2}$ are the weighted mean and variance determined for a population. For identical errors $\sigma_{i}$ for all the observations of a star, $\mu_{w}=\mu$ and $\sigma_{w}^{2}=\sigma^{2}$; this will be the case for most of the stars in our sample except for a few cases where outlying points influence the values. Here we used $\sigma_{w}^{2} \times \frac{N}{N-1}$ to approximate $S^{2}$, because $N \approx 122$ on average (i.e. large) in our sample of retained stars. The factor 3.289 enables one to estimate the amplitude (max min mag.) of the variations of a star considering that individual transits are distributed according to a Gaussian in the case of a constant star $(99.9 \%$ probability for a transit being comprised between $\min$ and $\max$ ). This will create a simple quantity that allows us to distinguish between variable and non-variable stars.

The estimation of the amplitude calculated here $(3.289 \times S)$ depends on the internal measurement errors for each transit. In fact, given that these errors are similar for a given star, and that our weighted $S$ was corrected for any outliers, $S$ depends on the median internal error for a given star. To use $S$ in our threshold calculations, we must first make sure that the internal error (I) depends only on one parameter: the magnitude of the star. Indeed, if it depends on anything else (spectral type, extension of the source etc...) calculating a threshold in the MagnitudeAmplitude domain makes no sense.

Figure 2 shows the distribution of the internal error (i.e. measurement error) of our sample with respect to $H p$ magnitude. One can easily see that Poisson errors dominate this plot. Moreover, the 3 intrinsic categories established in Sect. 5 are equally distributed. To make certain that the error depends only on the magnitude, we can make use of the variable called "external error / internal error" (Duquennoy et al. 1991) or $S / I$ in our case and check if it has been correctly estimated. The closer to 1 the $S$ / I variable is, the better the estimation of $I$. Of course, the 
Table 1. Selected stars.

\begin{tabular}{lcccccccc}
\hline \hline & $P$ & $U$ & $C$ & $D$ & $M$ & $R$ & “” & Total \\
\hline ampl. $\geq T_{\mathrm{h}}$ & 209 & 493 & 0 & 17 & 15 & 2 & 15 & 751 \\
$T_{1} \leq$ ampl. $<T_{\mathrm{h}}$ & 17 & 105 & 50 & 52 & 78 & 2 & 347 & 651 \\
ampl. $<T_{1}$ & 1 & 3 & 435 & 99 & 20 & 0 & 447 & 1005 \\
\hline Total & 227 & 601 & 485 & 168 & 113 & 4 & 809 & 2407 \\
\hline
\end{tabular}

Number of " $P$ ", " $U$ ", " $C$ ", " $D$ ", " $M$ ", " $R$ " and not-classified (" ") stars, above the positive threshold $\left(T_{\mathrm{h}}\right)$, between the positive and negative thresholds $\left(T_{\mathrm{h}}=T_{\mathrm{m}}+\operatorname{err}_{+}, T_{1}=T_{\mathrm{m}}-\right.$ err -$)$, and below both of them. $T_{\mathrm{m}}$ denotes the threshold calculated according to the method presented in Fig. 3.

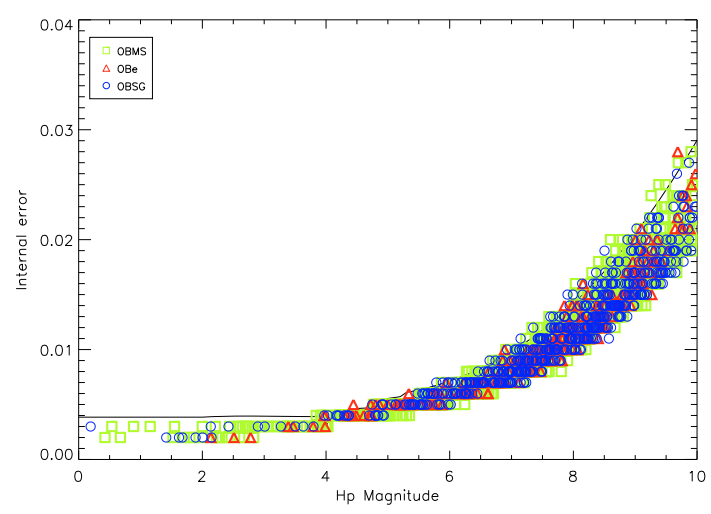

Fig. 2. Distribution of the internal error in our sample in the 3 intrinsic categories of stars from Sect. 5. Squares represent OBMS stars, triangles OBe stars and circles OBSG stars. Overplotted in black is the calculated threshold $T_{\mathrm{m}}$. Note that the shape of the $T_{\mathrm{m}}$-curve matches that of the internal error distribution, except for the brighter stars, which are less populated.

more a star is variable, the more $S / I$ exceeds 1 . When plotting the $S$ / I variable distribution in different bins of magnitude, we indeed see that the peaks stay relatively close to $S / I=1$ (error on $I$ is $\$ 10 \%$ in all cases), as confirmed by the overplotted threshold in Fig. 2.

Thus we can say that, although some scatter appears progressively at higher magnitudes (cf. Fig. 2; the scatter is $\sim$ identical in the 3 intrinsic categories), $I$ was correctly estimated for each star and depends only on the magnitude. The variations of $S$ should then follow those of $I$ relatively closely and it is now possible to calculate a threshold depending on the magnitude based on $S$, without doubt about the variability being falsely induced by different internal errors.

The indicative variability threshold from the HIPPARCOS consortium (ESA 1997, The Hipparcos and Tycho catalogs, Vol. 1, p. 52) would indicate that all the stars in our sample are variable, although some of them are classified as "constant" (cf. Table 1). This is probably due to the different estimator $(3.289 \times S)$ used for the computation of the amplitude. We calculated a threshold for our given sample by assessing the amplitude distribution in different magnitude bins. Despite the near-Gaussian distribution of the original magnitude measurements (for some test stars, the Kolmogorov-Smirnov test gives $\sim 80 \%$ probability that the measurements are drawn from a normally -distributed sample), the amplitudes of variability, being directly related to $S$, cannot be distributed according to a wellknown distribution. However, the squared amplitude, related to $S^{2}$, should be distributed according to a $\chi^{2}$ distribution with $N-1$ degrees of freedom, which asymptotically approaches a normal distribution for large $N$ ( $N$ being typically 122 in our sample).
To be more exact, we simulated bins with 10000 stars (the actual number of stars in each magnitude bin is actually closer to 100 , but we chose 10000 for greater reliability), with 100 transits each and compared the outcome to the actual distribution of the squared amplitude $\left(S^{2} \times\right.$ cst $)$ in each magnitude bin. Magnitudes for a constant star should be distributed according to a Gaussian centered on the mean magnitude, so the simulation represents what the distribution inside a given bin of magnitude should look like, if all the stars were constant. This is exactly what we are looking for, since variable stars should be located outside this simulated squared-amplitude distribution. We then adjusted the simulated squared-amplitude distribution to the real distribution and placed the variability limit $\left(t_{\mathrm{m}}, \mathrm{m}\right.$ for middle) at the point where the simulated distribution reaches $0.1 \%$ of the maximum. The error on this limit is represented by the $F W H M=$ $2.35 \times \sigma_{\text {err }}\left(\approx\right.$ Gaussian because order $\left._{\chi^{2}}=121\right)$ of the adjusted simulated distribution. The errors on the calculated $t_{\mathrm{m}}$ are transformed to $t_{1}$ and $t_{\mathrm{h}}$ with $t_{1, \mathrm{~h}}=t_{\mathrm{m}} \pm 3 \times \sigma_{\mathrm{err}}=t_{\mathrm{m}} \pm$ err. Samplesimulated and real distributions of the squared-amplitude are represented in Fig. 3, where the distributions for the 3 different classes of intrinsic variability presented in Sect. 5 have been overplotted. The error err on $t_{\mathrm{m}}$ is then transformed into the error on $T_{\mathrm{m}}\left(T_{\mathrm{m}}^{2}=t_{\mathrm{m}}\right)$ to be representable in a more classical way in Fig. 4. Note that err becomes err - and err $_{+}$in the amplitude domain, i.e. the error is asymmetrical.

A fourth order polynomial is adjusted to the points in each bin of magnitude to obtain the resulting thresholds presented in Fig. 4, where the 3 lines represent the threshold $T_{\mathrm{m}}$ minus the error to the lower side ( $T_{1}$, lower dash-dotted line), the threshold $T_{\mathrm{m}}$ itself (solid line) and $T_{\mathrm{m}}$ plus the error to the higher side $\left(T_{\mathrm{h}}\right.$, upper dash-dotted line). We chose $T_{\mathrm{h}}$ as a conservative measure of the variability threshold, i.e. the 751 stars above $T_{\mathrm{h}}$ are assumed to be variable. Between $T_{1}$ and $T_{\mathrm{h}}$ lie "possibly variable stars", while supposedly "non variable" stars are located below $T_{1}$. Table 1 presents the results in a general manner. As one can see, there is a negligible fraction $(<0.5 \%)$ of known periodic or variable stars below the established lower limit $\left(T_{1}\right)$. Moreover, no stars deemed as constant by the HIPPARCOS consortium appear above the highest threshold $T_{\mathrm{h}}$, thus validating "a posteriori" our calculations. Most of the "M" stars are located in the "possibly variable" zone which is normal, considering the small amplitude of their variations. "D" stars are mostly classified as "non-variable", a result that is also encouraging, i.e. the number of stars wrongly classified as variable is minimal. In addition, less than $2 \%$ of the total number of stars which could not be classified lie above the threshold $T_{\mathrm{h}}$.

We assume all stars above $T_{\mathrm{h}}$ to be variable, hence (per our choice of $T_{\mathrm{h}}$ ) the probability of having a non-variable star among the candidates is less than $0.1 \%$ as confirmed by the statistics (cf. Table 1). The probability of finding a variable star (with amplitude Amp. $\gtrsim 0.03 \mathrm{mag}$ ) below $T_{1}$ is $\sim 1 \%$, as indicated by the quasi absence of "U" or "P" stars, while it is around $15 \%$ for 

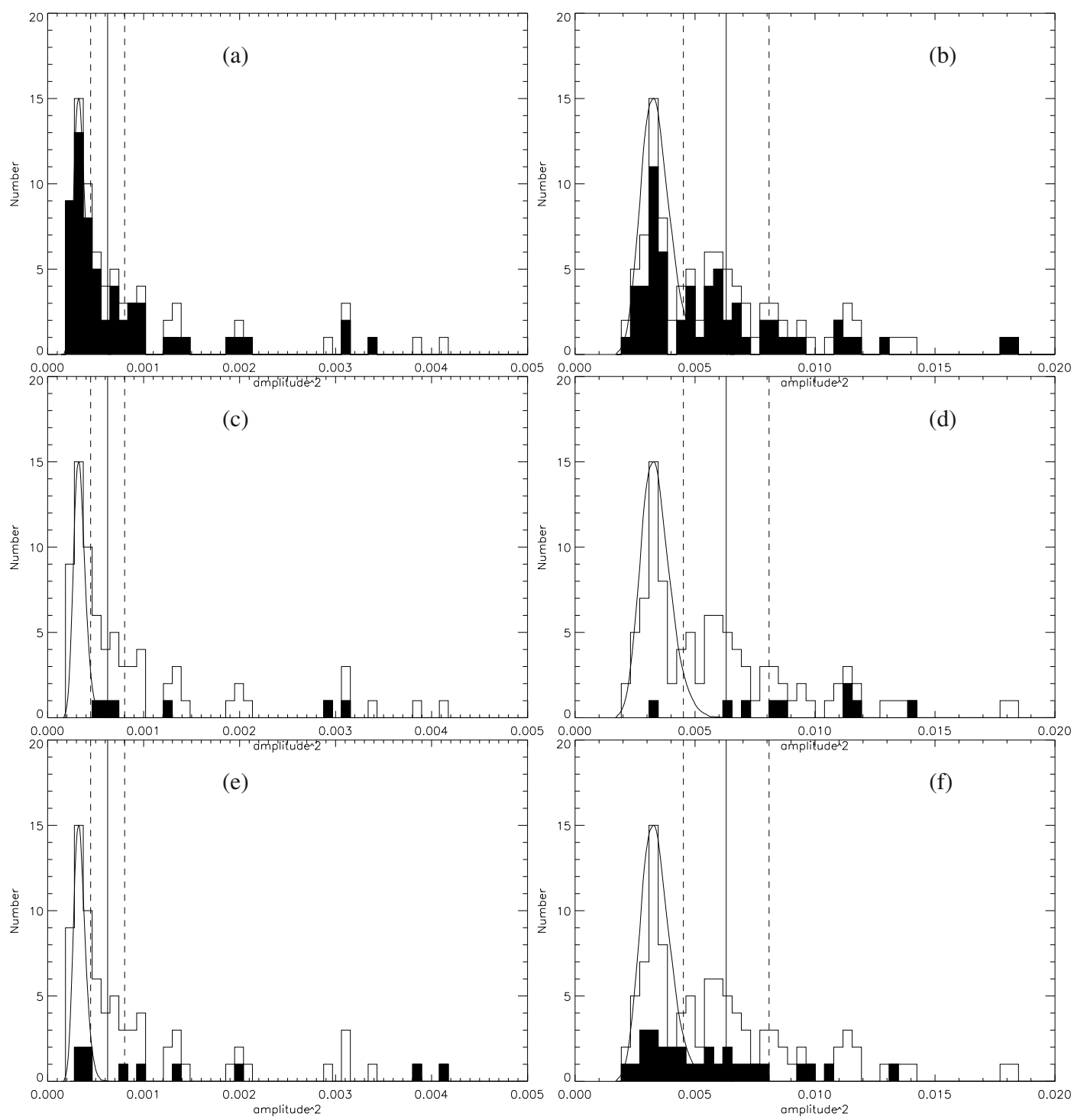

Fig. 3. Comparison between the distribution in bins of magnitude and the simulated results for the whole sample of OB stars superimposed in black with the 3 intrinsic categories from Sect. 5. Bins are for $H p=[5,5.5](l e f t)$, and $H p$ [9, 9.25] (right). From top to bottom, the filled histograms represent the number of OBMS, OBe and OBSG stars in the above-mentioned magnitude bins. Note that there are a few points with a squaredamplitude that is off-scale in these diagrams; these have not been shown for reasons of clarity. The solid line represents $t_{\mathrm{m}}$ while the dotted lines represent $t_{1}$ and $t_{\mathrm{h}}$. The thresholds calculated in each category separately correspond (within the errors) to the combined threshold in both the $S$ and $S / I$ cases. As can be seen from panels $\mathbf{c}$ ) and $\mathbf{d})$, OBe stars are less numerous.

stars with a relatively low amplitude (Amp. $\lesssim 0.03 \mathrm{mag}$ ). On the other hand, when considering stars below $T_{\mathrm{h}}$, the probability of finding a variable star rises to $\sim 10 \%$ for Amp. $\gtrsim 0.03$ mag and $\sim 70 \%$ for Amp. $\$ 0.03$ mag. That is the reason why stars located in this particular region have been classified as "possibly variable stars". It comprises more than $80 \%$ of the microvariable stars and about $15 \%$ of the "U" and "P" stars (cf. Table 1). The error on the magnitude is $\sim 0.002 \mathrm{mag}$ on average and depends on the number of points for each star and on the variability level. Some shifts in magnitude in Fig. 4 could modify the distribution around the threshold by a few percent $(\$ 5 \%)$, below the limiting magnitude $\left(H_{\mathrm{p}}=8 \mathrm{mag}\right)$ and more above it, but this is within the error margins in the statistics of Table 2 , which are quite large.

It is important to stress that the "S/I" method from Duquennoy et al. (1991) also provides us with the means to verify a posteriori the accuracy of the calculated threshold, i.e. the distribution of stars above and below the threshold. Indeed, the introduction of a variable $\chi^{2}=(N-1) \times\left(S^{2} / I^{2}\right)$ (Duquennoy et al. 1991) allows us to calculate a threshold $\left(T_{S / I}\right)$ which does not depend on the magnitude. Applying a false probability detection of $0.1 \%$ for this threshold, we find that the distribution of stars above and below $T_{S / I}$ is similar to the distribution above and below $T_{\mathrm{h}}$ within less than $5 \%$. Of course, this is not surprising considering the fact that we established that the internal error $I$ was correctly estimated and thus the shape of the internal error seen in Fig. 2 is the same $(\times 3.289)$ as that of $T_{\mathrm{m}}$ (cf. Figs. 2 and 4).

\section{Analysis of the variability}

The 751 stars selected as "variable" thanks to our determined threshold were then analyzed with the CLEAN algorithm (Scargle 1982; Roberts 1987) followed by a Phase Dispersion Minimization technique (Stellingwerf 1978, PDM). For the 


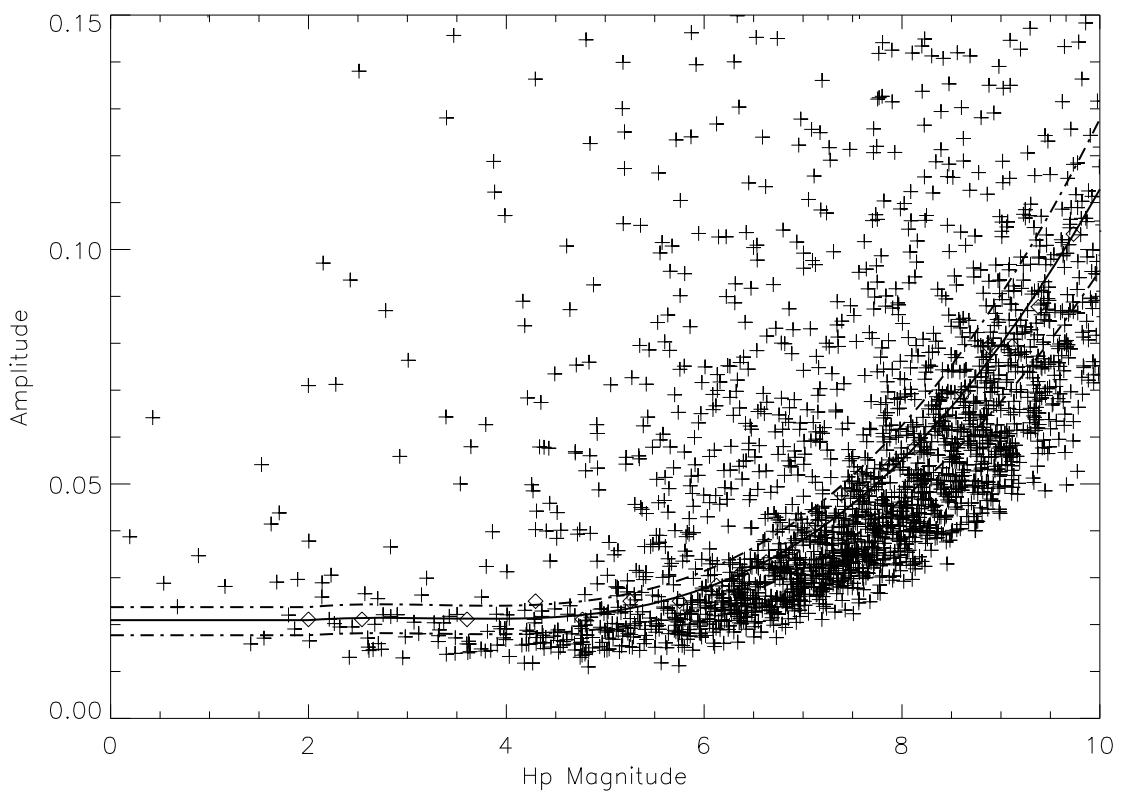

Fig. 4. Variability threshold calculated for our sample. $T_{\mathrm{m}}$ (solid line) corresponds to the point where the distribution reaches $0.1 \%$ of its maximum, $T_{\mathrm{h}}$ (upper dash-dotted curve) corresponds to the higher error limit and $T_{1}$ (lower dash-dotted curve) corresponds to the lower error limit.
CLEAN program we used a gain of 0.2 and a maximum number of 250 iterations. We calculated a false alarm probability (fap) threshold for each star and kept the three biggest power peaks of the CLEAN spectrum, only if they were above a 99\% fap threshold. We then used the PDM around each of those peaks to locate the position more precisely.

\section{Results and discussion}

Of the 227 previously known periodically varying stars (flagged "P"), about $90 \%$ were found to be periodic in this study, some with revised periods. The $10 \%$ discrepancy between HIPPARCOS and this study most probably comes from the difference in the number of points in the analysis. The HIPPARCOS consortium excluded all the flagged points while we kept some of them, hence the difference.

We chose to classify the supposedly variable stars in 4 broad categories. These include, first under intrinsic variables: OB stars of luminosity classes I and II (OBSG), Main sequence (III to V) stars with no emission (OBMS) and OB stars in luminosity classes III to $\mathrm{V}$ with emission (OBe stars) and then under extrinsic variables: eclipsing binaries (E). Some eclipsers may reveal other types of variability but the eclipse phenomenon usually dominates, so we will retain them only in the E category. As for types of variability, we follow the definition in the General catalog of Variable Stars (GCVS, Samus et al. 2004) and of Gautschy \& Saio (1996). The principal variability types used are listed in Appendix A. Note that stars for which the lightcurve is not precise enough to decide between an ellipsoidal variable and an eclipsing binary, were given preference as eclipsing binaries.

We found 169 stars with new periods, of which $\approx 50 \%$ were also found by Koen \& Eyer (2002). Included in these new periodically varying stars, are 5 new eclipsing binaries of which 1 was also in Koen \& Eyer (2002) and 2 were found by Otero (2003). The phase plots of stars with new periodicities are given in Appendix B (see Fig. 5). When classifying the variability types, all of the probable periods have been taken into account; we made no assumptions on the veracity of those periods. However, periods of $P \approx 0.089 \mathrm{~d}$ are probably related to the orbital period of the HIPPARCOS satellite as discussed in Koen \& Eyer (2002), so they have not been taken into account when classifying the types of variability. To indicate any "unsure" status of the new periods, we added question marks to the variability types (Appendix A tables).

Our complete sample of OB stars consists of 127 eclipsing (or supposed as eclipsing) binaries, 229 OBe stars, 1482 OBMS stars and 577 OBSG stars. The statistics for all the categories are presented in Tables 2 (intrinsic categories) and 3 (eclipsing binaries), while the details are presented in a separate table in Appendix A. The errors on the proportions in Tables 2 and 3 are based on the assumption that the numbers of stars in a given category $\left(N_{i}\right.$ compared to $\left.N_{\text {tot }}\right)$ arise from a Binomial distribution (which tends towards a Poisson distribution for $\left.N_{\text {tot }} \gg 10\right)$, hence $\sigma_{\text {binomial }}=\sqrt{N_{\text {tot }} p_{i}\left(1-p_{i}\right)}$, which gives an estimated error margin with a confidence interval of $99 \%$ of $E_{99 \%}=2.58 \times \sqrt{N_{\text {tot }} p_{i}\left(1-p_{i}\right)}$, and an error in percent of $E_{99 \%}(\%)=\frac{E_{99 \%}}{N_{\text {tot }}}=2.58 \times \sqrt{\frac{p_{i}\left(1-p_{i}\right)}{N_{\text {tot }}}}$, where $p=\frac{N_{i}}{N_{\text {tot }}}$ is the proportion and $N_{\text {tot }}$ the number of stars in the chosen sub-sample.

Moreover, we recalculated the proportions taking the 7 previously known periodically varying stars that were deleted because of too many flagged points and concluded that their influence is negligible, as can be seen from the row labeled "Not included" in Table 2. Note that the HIPPARCOS satellite scans along an axis with a "scan-data angle" depending on the time of observation. Thus, the more a source is extended, the more the variation of this angle can induce false variability. This angle is mainly sensitive to extended sources and nebulosities. This will be discussed in Sect. 5.2.

One can see from Table 2 that OBe stars are much more variable intrinsically than OBSG or OBMS stars, and also more variable than the complete sample (line labeled " $\mathrm{OB}_{i, v}$ vs. tot. $\left.\mathrm{OB}_{i}{ }^{\prime \prime}\right)$. On the other hand, OBMS stars are below $T_{\mathrm{h}}$ in $83 \%$ of cases, which means they are mostly non variable (53\% are classified as non-variable, against $11 \%$ for the OBe stars and $34 \%$ for the OBSG stars). Figure 6 also shows these aspects in a different way. One can see in that figure that OBe stars are overrepresented above the threshold $T_{\mathrm{h}}$. This will be developed in Sects. 5.1 and 5.2. 

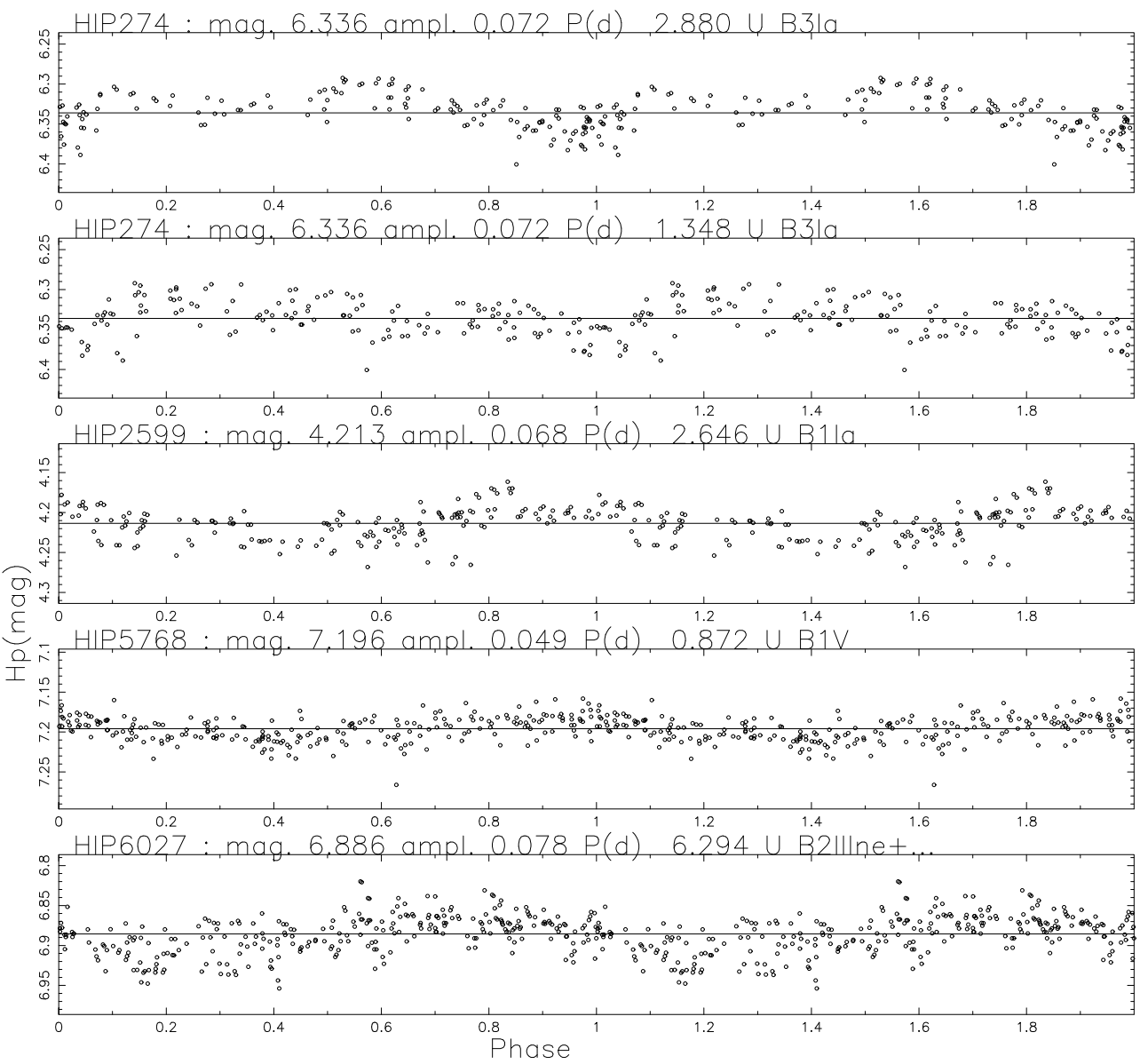

Fig. 5. This figure is a representative extraction of our "catalog of New Periodic stars" given in Appendix B2. It plots $H p$ mag. vs. Phase. The epoch is arbitrarily taken as JD-2 $440000=7800$, which is the beginning of the HIPPARCOS observations. On top of each lightcurve are the HIPPARCOS number, the $H p$ magnitude and mean amplitude calculated in this study, the period, variability flag and spectral type from the HIPPARCOS data (periods can also be new ones). The $H p$ magnitude, mean amplitude and periods are accurate to $\sim 0.1 \%$.

Table 2. OB Stars in the 3 OB categories and corresponding statistics.

\begin{tabular}{lccccc}
\hline \hline$N_{i}:$ & OBMS (\%) & OBe (\%) & OBSG (\%) & Ecl. (\%) & $N_{\text {tot }}(\%)$ \\
\hline ampl. $\geq T_{\mathrm{h}}$ & $256(34 \pm 4.5)$ & $172(23 \pm 4)$ & $201(27 \pm 4)$ & $122(17 \pm 3.5)$ & $751(31 \pm 2.5)$ \\
$T_{1} \leq$ ampl. $<T_{\mathrm{h}}$ & $442(68 \pm 5)$ & $32(5 \pm 2)$ & $177(27 \pm 4.5)$ & & $651(27 \pm 2)$ \\
ampl. $<T_{1}$ & $782(78 \pm 3)$ & $25(2 \pm 1)$ & $198(20 \pm 3)$ & $(1)$ & $1005(42 \pm 2.5)$ \\
\hline Total category & $\mathbf{1 4 8 0}(61 \pm 2.5)$ & $\mathbf{2 2 9}(10 \pm 1.5)$ & $\mathbf{5 7 6}(24 \pm 2)$ & & 2407 \\
\hline Not included & 2 & 0 & 1 & $(4)$ & 7 \\
Total category & $\mathbf{1 4 8 2}(61 \pm 2.5)$ & $\mathbf{2 2 9}(10 \pm 1.5)$ & $\mathbf{5 7 7}(25 \pm 2)$ & & \\
\hline Statistics $(\%)$ & & & & & \\
OB $_{i, v}$ vs. tot. $\mathrm{OB}_{i}$ & $17 \pm 2.5$ & $75 \pm 7$ & $35 \pm 5$ & \\
OB $_{i, b}$ vs. tot. $\mathrm{OB}_{i}$ & $83 \pm 2.5$ & $25 \pm 7$ & $65 \pm 5$ & \\
OB $_{i, p v}$ vs. tot. $\mathrm{OB}_{i}$ & $30 \pm 3$ & $14 \pm 6$ & $31 \pm 5$ & \\
OB $_{i, n v}$ vs. tot. $\mathrm{OB}_{i}$ & $53 \pm 3$ & $11 \pm 5$ & $33 \pm 5$ & & \\
$\mathrm{OB}_{i, v}$ vs. $\mathrm{OB}_{v}$ & $46 \pm 4.5$ & $24 \pm 4$ & $30 \pm 4$ & & \\
$\mathrm{OB}_{i, b}$ vs. $\mathrm{OB}_{b}$ & $74 \pm 3$ & $3 \pm 1$ & $23 \pm 2.5$ & & 1656 \\
\hline
\end{tabular}

$\mathrm{OB}_{i}$ corresponds to the 3 intrinsic categories, $i$ being OBMS, OBe or OBSG. The index $v$ stands for "variable" (above $T_{\mathrm{h}}$ ), $b$ means below $T_{\mathrm{h}}$, $n v$ stands for "non-variable" and $p v$ stands for "possibly variable". In the cases "A vs. B", $B=\mathrm{OB}_{i}$ corresponds to $N_{\text {tot }}=1480$, 229 or 576 ; while A corresponds to $N_{i}$.

\subsection{Extrinsic variability: eclipsing binaries}

All the eclipsing binary lightcurves are presented in Appendix B, an extraction of which is presented here in Fig. 7. They are also summarized, along with their variability types, in Appendix A, of which Table 4 is the beginning. When taking into account only the stars down to $H p=8$, the statistics from Table 3 do not vary significantly (i.e., the proportions are identical).

Figure 8 presents the periods and amplitudes of variation for the eclipsing binaries in the 3 classes of intrinsic variability. The amplitude of Fig. 8 is representative of the depths of the eclipses, 

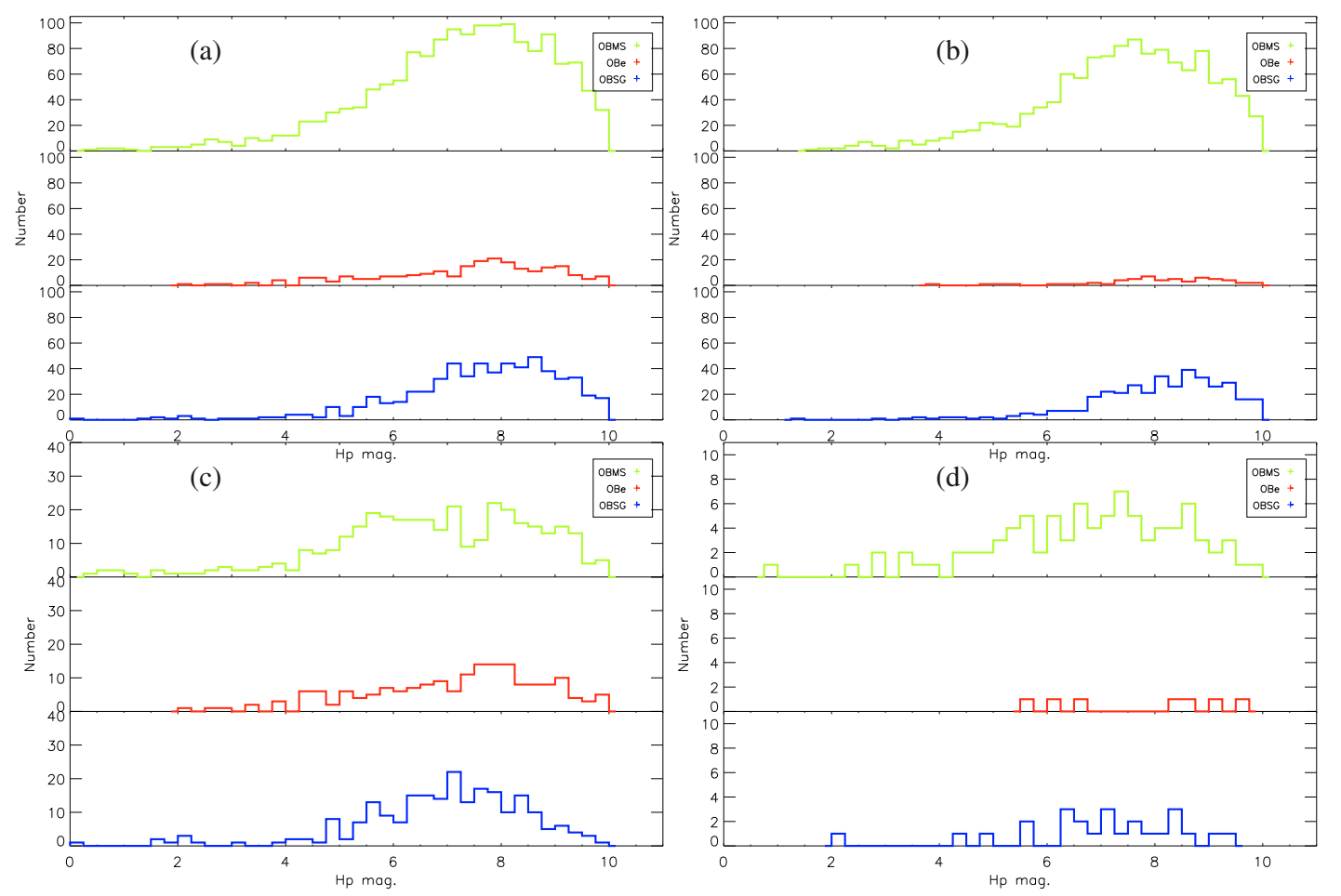

Fig. 6. The four panels of this figure represent the distribution in magnitude for the 3 intrinsic variability classes in 4 different situations. From top to bottom, OBMS stars, OBe stars and OBSG stars. a) The whole sample of 2407 stars, b) the 1656 stars below $\left.T_{\mathrm{h}}, \mathbf{c}\right)$ the 751 stars above $T_{\mathrm{h}}$ and d) the 127 eclipsing binaries.

Table 3. OB eclipsing Stars in the 3 OB categories and corresponding statistics.

\begin{tabular}{|c|c|c|c|c|}
\hline$\overline{N_{i}:}$ & OBMS (\%) & OBe (\%) & OBSG (\%) & $N_{\text {tot }}$ \\
\hline$\overline{\overline{\text { Ecl. binaries }}}$ & $89(73 \pm 10)$ & $\overline{7(6 \pm 5)}$ & $26(21 \pm 9)$ & $\overline{122}$ \\
\hline $\mathrm{OB}_{i, e}$ vs. tot. $\mathrm{OB}$ & $4 \pm 1$ & $0.3 \pm 0.3$ & $1 \pm 0.5$ & $5.3 \pm 1.2$ \\
\hline $\mathrm{OB}_{i, e}$ vs. tot. $\mathrm{OB}_{i}$ & $6 \pm 2$ & $3 \pm 3$ & $4 \pm 2$ & \\
\hline $\mathrm{OB}_{i, e}$ vs. $\mathrm{OB}_{i, v}$ & $28 \pm 6$ & $3.9 \pm 3.8$ & $10 \pm 5$ & \\
\hline Not included & 5 & 0 & 0 & 5 \\
\hline Total category & $94(74 \pm 10)$ & $7(6 \pm 5)$ & $26(20 \pm 9)$ & 127 \\
\hline
\end{tabular}

$\mathrm{OB}_{i}$ corresponds to the 3 intrinsic categories, $i$ being OBMS, OBe or OBSG. The index $v$ stands for "variable" (above $T_{\mathrm{h}}$ ). The line "Not included" refers to the stars that were not included in the analysis because of too many flagged points, or below $T_{\mathrm{h}}$.

but not as precisely as for other types of variability. Indeed, the estimation of the peak-to-peak amplitude works better the more the lightcurve approaches a sinusoid. In the case of a detached system, where the primary and the secondary minima are very different, the accuracy of the estimation of the amplitude strongly depends on the number of points during the eclipses versus those between.

Table 4 also shows that there are twice as many contact (EB) systems as detached (EA) among the OBMS stars, while there are about as many contact as detached systems among OBSG stars. This will be discussed in Sect. 5.2 in their respective categories although this is not an intrinsic variability. Note the difference in the peaks of the period histograms between the OBSG and the OBMS stars. The orbital periods seem to be larger for OBSG stars on average. This difference is highly significant ( $p=$ $99 \%$ ) according to the Wilcoxson criterion (Wilcoxon 1945).
It is also seen when considering the sample to the limit of completeness (i.e. below $H p=8 \mathrm{mag}$ ).

\subsection{Intrinsic variability}

Figure 9 presents the periods and amplitudes of variation in the 3 classes of intrinsic variability. Periods in the 3 classes appear equally distributed in this figure, although if we consider only the periods already found by HIPPARCOS (allowing for the fact that our periods might not be reliable) the peak from the OBSG class seems to occur at a slightly longer period than the OBMSclass peak ( $8 \mathrm{~d}$ compared to $3 \mathrm{~d}$ ). This could be easily explained by the well-known period-mass relation: the greater the mass of the star, the larger its radius, the lower its mean density, and therefore the longer its pulsation period. This relation should basically apply to all the OB stars studied here. Moreover, there seems to be a gap in Fig. $9 \mathrm{a}$ and b between $P=0.3 \mathrm{~d}$ and $P=1 \mathrm{~d}$, whose origin remains unknown.

Note that the first and second panels of Fig. 9 take the new periods into account, while the third and fourth do not. This is because some of the stars have several periods which are associated with identical amplitudes and magnitudes. The total numbers of stars are 56, 7 and 29 for the OBMS, OBe and OBSG categories, respectively, in the amplitude histogram (lower left panel), while they reach 108, 39 and 121 in the period histogram (upper right panel), because they include all the possible new periods. It is interesting in this case to note that the total number of OBe periods is more than five times higher if we add the new periods (number of new/old $=5.5$ ). On the other hand, the total number of OBSG periods (including new periods) is about 4 times the initial number of OBSG periods, while the number of new periods found in the OBMS stars class is modest $($ new/old $=1.9)$. 

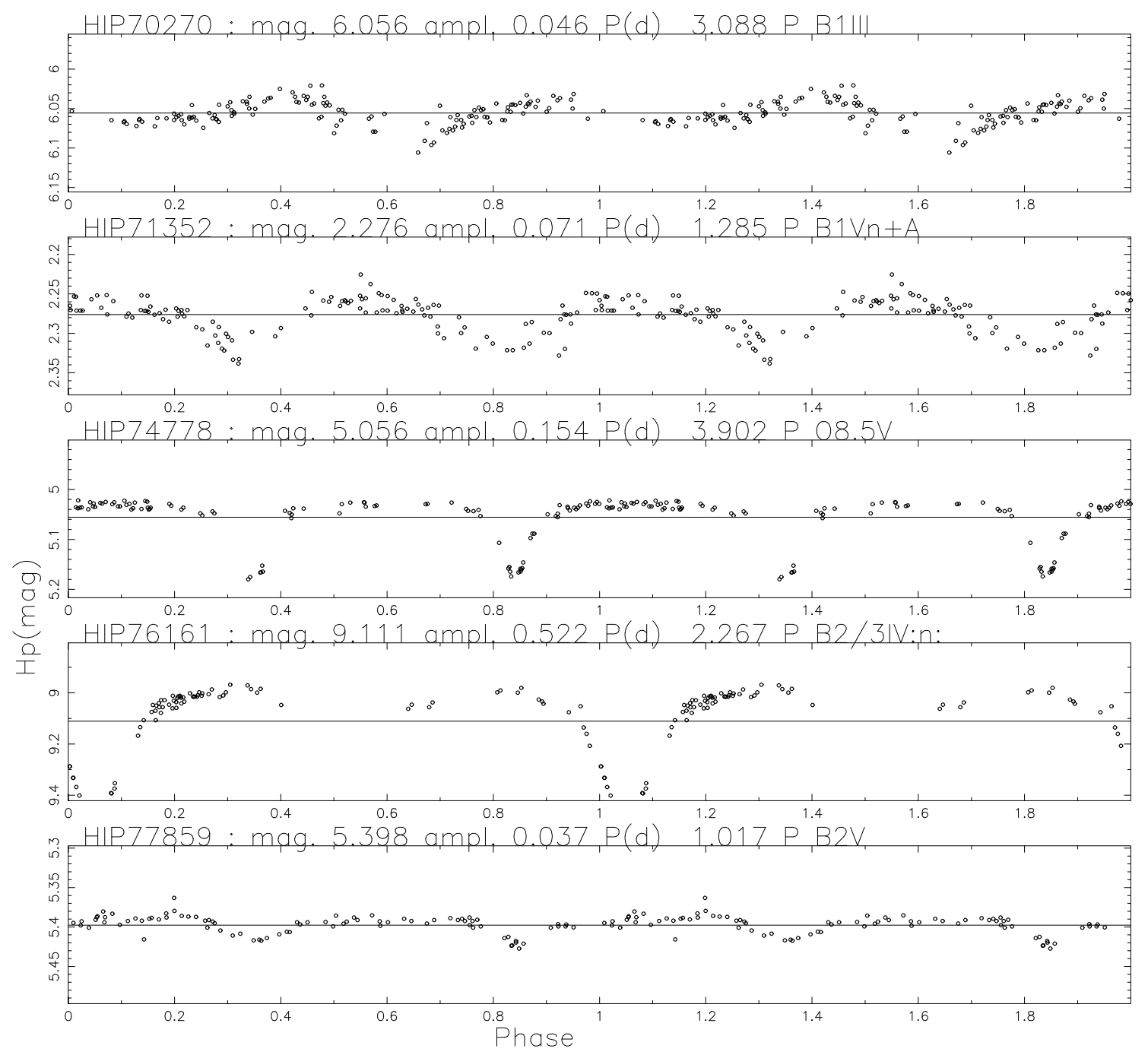

Fig. 7. This figure is the beginning of our "catalog of Eclipsing Binaries" given in Appendix B1. It contains 122 candidates; the remaining 5 are in Appendix B3 and B4. It plots $H p$ mag. vs. Phase. The epoch is arbitrarily taken as JD-2440 $000=7800$, which is the beginning of the HIPPARCOS observations. On top of each lightcurve are the HIPPARCOS number, the $H p$ magnitude and mean amplitude calculated in this study, the period, variability flag and spectral type from the HIPPARCOS data (periods can also be new ones). The $H p$ magnitude, mean amplitude and periods are accurate to $\sim 0.1 \%$.

Table 4. OB eclipsing binaries of the HIPPARCOS catalog.

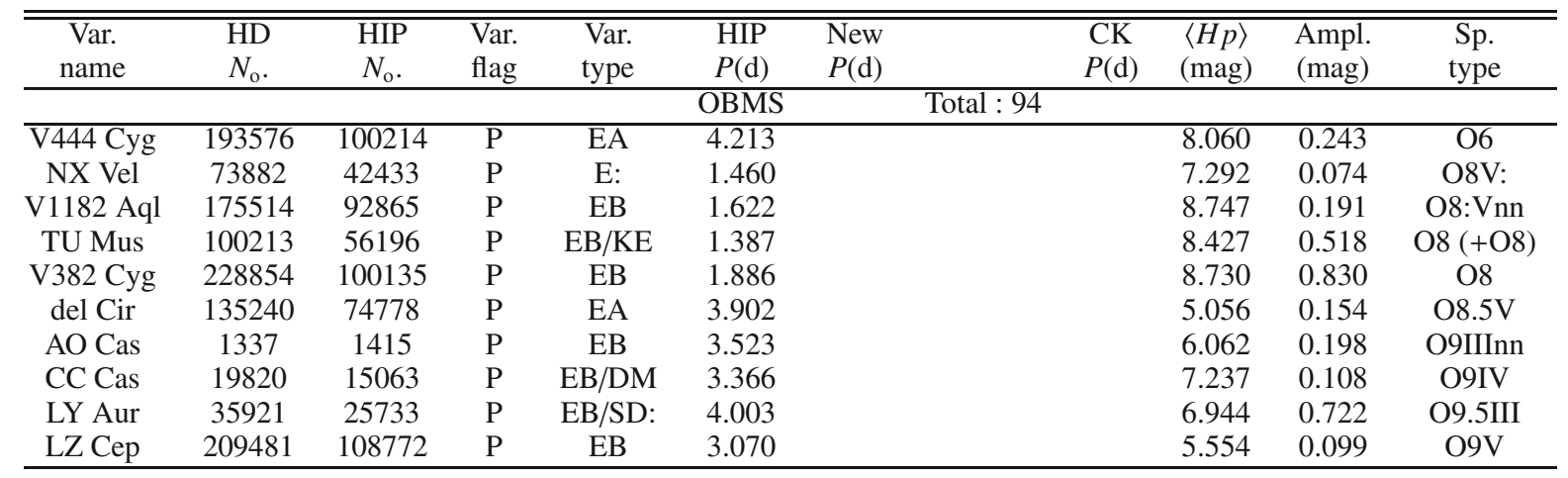

Table 4 is presented in its entirety in Appendix A. The columns are: the variable name, the HD number, the HIPPARCOS number, the HIPPARCOS variability flag, the variability type we assigned in this study, the HIPPARCOS period (if any), the new periods found in this study ( 2 columns, for a possible alternative period), the periods found by Koen \& Eyer (2002) (if any), the $H p$ magnitude, the mean amplitude calculated here according to Eq. (1) and the spectral type according to the Vizier database. 

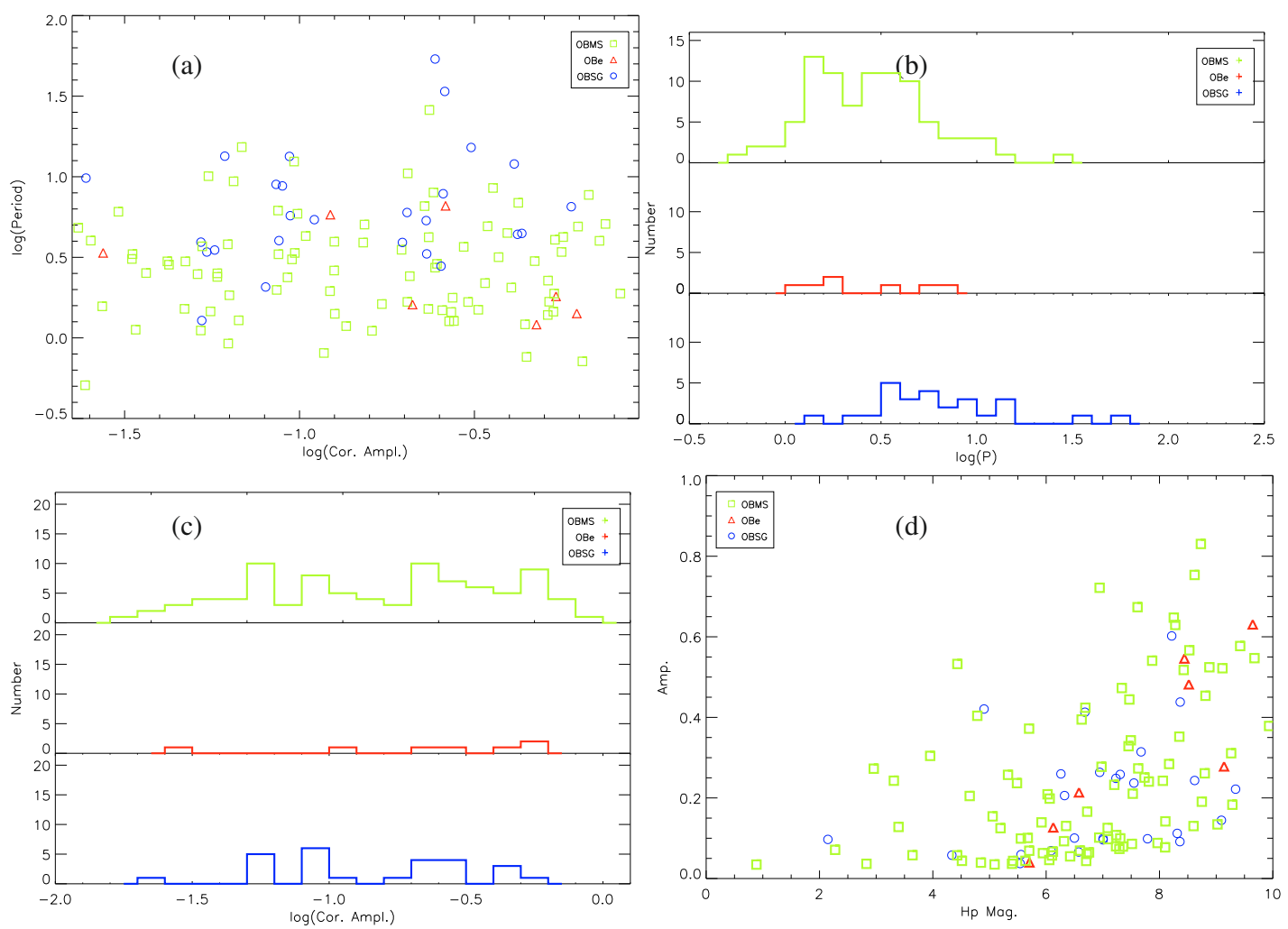

Fig. 8. Eclipsing binaries: the upper left panel a) presents the log of the period (days) versus the log of the amplitude of the variations, while the upper right panel b) presents the histograms of the periods in the 3 intrinsic variability classes OBMS, OBe and OBSG. Squares represent OBMS stars, triangles OBe stars and circles OBSG stars. The lower left panel c) presents the histograms of the amplitude in the 3 intrinsic variability classes. The lower right panel $\mathbf{d})$ presents the amplitude vs. Hp magnitude.

\subsubsection{OB Main Sequence stars}

Not surprisingly, OBMS stars represent the greater part of our OB sample of stars $(65 \%)$. Their variability classification is presented in Table 5. As can be seen in Table 2, less than $20 \%$ of them are classified as variable in this study, which is rather low compared to the percentage of variable OBe and OBSG stars (OBe: $75 \%$, OBSG: $35 \%$ ). In fact, about $30 \%$ of all the stars in the sample are supposedly variable, and the proportion of variable OBMS stars is below that average. This is easily explained when one considers the fact that main sequence stars are much more stable than the two other categories involved here. There also seem to be more eclipsing systems among this class (cf. Table 3). It is not impossible that this is due to the fact that the stability of these stars prevents them from merging as often as the stars in the other categories, hence the higher number of non-merged eclipsing binaries (Podsiadlowski 2006). Moreover, despite the limited sample, there is a trend towards more EB (contact) systems than EA (detached). Indeed there are $\approx 60 \%$ EB eclipsing binaries compared to $\approx 30 \%$ EA eclipsing binaries (the rest are classified as E or EW). The statistics stay the same if we consider only the stars with $H p \leq 8$ mag. This might be related to the fact that OBMS stars in binary systems are closer together than the stars in our other categories, and thus easier to detect (i.e., the inclination angle can deviate more from $90^{\circ}$, because the components are closer).

\subsubsection{OBe stars}

The variability classification of OBe stars is presented in Table 6 . We mention that the $\mathrm{OBe}$ stars we defined here are in fact $\mathrm{Oe}$ and
Be stars. Oe stars were first introduced by Conti \& Leep (1974), and usually fall inside the range O9-B0. However, Negueruela et al. (2004) mention that some published spectral types are too early most likely due to filling in of HeI lines. That is why we also included spectral types earlier than O9. Although Oe stars do not show the presence of a disk as in Be stars they are considered to be the extension of the Be stars to the $\mathrm{O}$ type stars.

As noted earlier, OBe stars seem to be by far the most variable class in our 3 intrinsic categories. Indeed the incidence of rapid rotation and pulsations and their possible interactions could explain this high level of variability. These hypotheses are strengthened by the apparent correlation found between pulsations and an outburst in the COROT observations of the B0e star HD 49330 (Huat 2009). Moreover, OBe stars are nearly absent below the threshold $T_{\mathrm{h}}$ and the cause may be that even if the variability is not periodic, a few outbursts might have been picked up by HIPPARCOS observations and lead to high amplitude variability. As for the variability of the "scan-data angle" noted earlier, Quirrenbach et al. (1997) note that the disks, if present, have negligible (for the HIPPARCOS satellite optics) extensions in the optical of a few milli-arcseconds. So this cannot account for variations seen in OBe stars. It does not apply, though, to any flux variations due to the instabilities in the disks which can augment the variability of the star.

In addition, a good fraction (20-60\%) of OBe stars are supposed to be in a post mass-transfer phase of a close binary system (Pols et al. 1991). The upper right panel of Figure 8 seems to indicate that $\mathrm{OBe}$ star periods do not reach as high as OBMS star periods. However, when taking into account the actual numbers with a Wilcoxon test (7-OBe against 89-OBMS EB Periods), the 

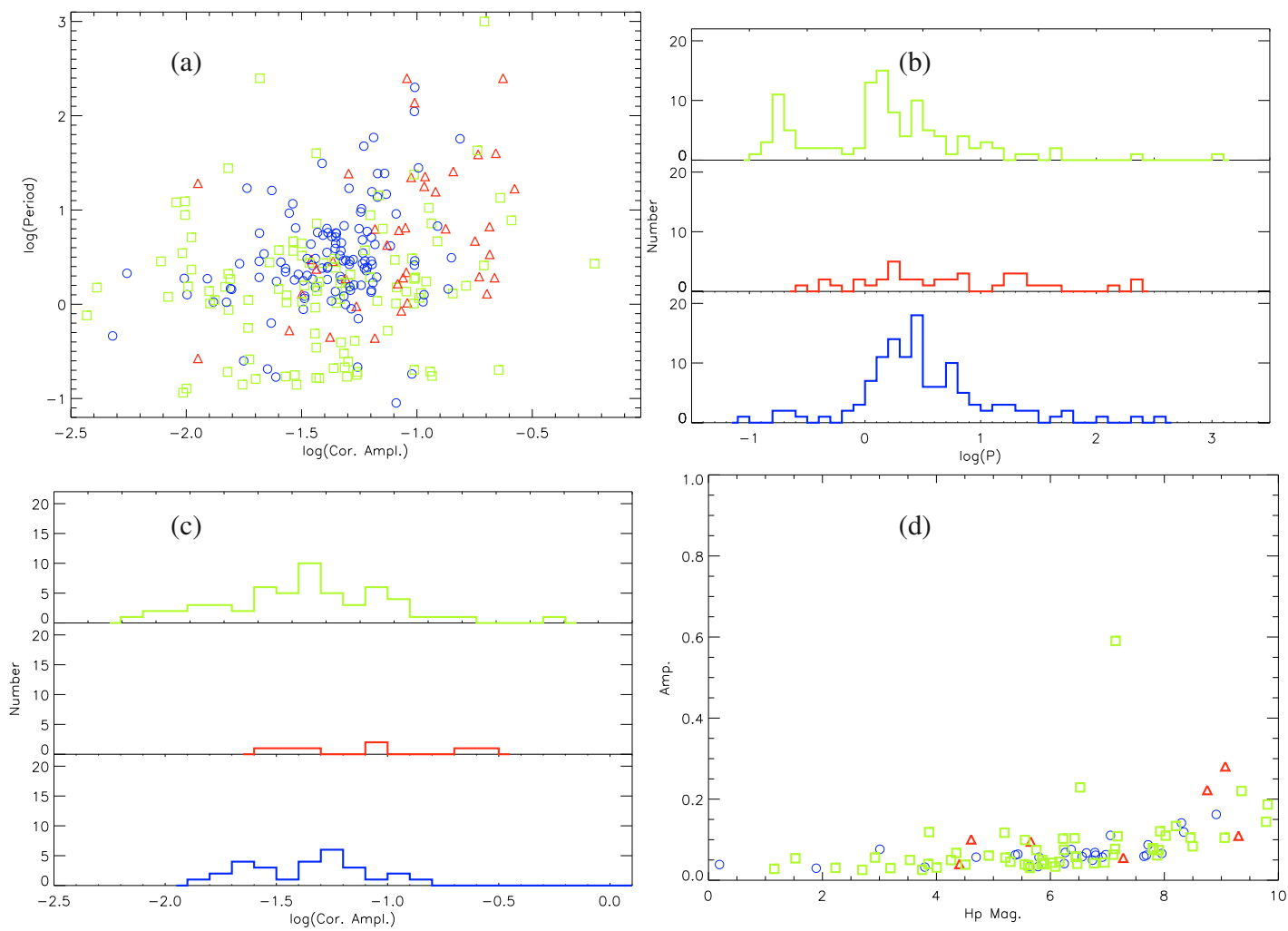

Fig. 9. Variable stars: from top to bottom, left to right. Colors and symbols are the same as in Fig. 8. The first panel a) presents the log of the period (days) versus the amplitude of the variations, while the second panel b) presents the histograms of the periods in the 3 intrinsic variability classes of OBMS, OBe and OBSG stars (from bottom to top). The third panel c) presents the amplitude histograms of the same 3 classes of variability, and the fourth $\mathbf{d}$ ) is the same as Fig. 4 for the periodic stars above $T_{\mathrm{h}}$.

Table 5. OBMS stars above the threshold $T_{\mathrm{h}}$.

\begin{tabular}{|c|c|c|c|c|c|c|c|c|c|c|}
\hline $\begin{array}{c}\text { Var. } \\
\text { name }\end{array}$ & $\begin{array}{l}\mathrm{HD} \\
N_{\mathrm{o}} .\end{array}$ & $\begin{array}{c}\mathrm{HIP} \\
N_{\mathrm{o}} .\end{array}$ & $\begin{array}{l}\text { Var. } \\
\text { flag }\end{array}$ & $\begin{array}{l}\text { Var. } \\
\text { type }\end{array}$ & $\begin{array}{l}\text { HIP } \\
P(\mathrm{~d})\end{array}$ & $\begin{array}{l}\text { New } \\
P(\mathrm{~d})\end{array}$ & $\begin{array}{l}\text { CK } \\
P(\mathrm{~d})\end{array}$ & $\begin{array}{c}\langle H p\rangle \\
(\mathrm{mag})\end{array}$ & $\begin{array}{l}\text { Ampl. } \\
\text { (mag) }\end{array}$ & $\begin{array}{l}\text { Sp. } \\
\text { type }\end{array}$ \\
\hline & 97950 & 54948 & $\mathrm{D}$ & IA & & & & 9.019 & 0.114 & O5 \\
\hline \multirow[t]{3}{*}{ S Mon } & 47839 & 31978 & U & SR/L? & & 3.271 & & 4.551 & 0.040 & $\mathrm{O} 7$ \\
\hline & 91824 & 51773 & & IA & & & & 8.129 & 0.068 & $\mathrm{O} 7$ \\
\hline & 152623 & 82876 & D & IA & & & & 6.726 & 0.043 & $\mathrm{O} 7$ \\
\hline NSV 08060 & 152723 & 82936 & U & BCEP? & & 0.395 & & 7.150 & 0.065 & O7 \\
\hline \multirow[t]{2}{*}{ gam02 Vel } & 0 & 39953 & $\mathrm{U}$ & BCEP? & & 1.384 & & 1.705 & 0.044 & O8III \\
\hline & 96670 & 54358 & U & IA & & & & 7.467 & 0.055 & O8 \\
\hline \multirow[t]{2}{*}{ V871 Cen } & 101205 & 56769 & U & IA & & & & 6.474 & 0.092 & O8var \\
\hline & 124314 & 69628 & D & IA & & & & 6.696 & 0.040 & O8 \\
\hline V1809 Cyg & 203064 & 105186 & U & $\mathrm{L}$ ? & & & & 5.022 & 0.031 & O8 \\
\hline
\end{tabular}

Table 5 is presented in its entirety in Appendix A.

difference between both distributions is significant only to a level of $60 \%$ (Wilcoxon 1945; Mann \& Whitney 1947). It would be of considerable interest to see if this tendency is confirmed in a larger sample of OBe binary systems.

\subsubsection{OB supergiants stars}

OB supergiants are usually thought to be more prone to variability, mainly because of their large radii, thus weaker surface gravity, and subsequent tendency to be unstable. When we take the total sample of stars, we find that about $30 \%$ of the variable stars (above $T_{\mathrm{h}}$ ) are OBSGs, while only about $20 \%$ of the non-variables (below $T_{\mathrm{h}}$ ) are OBSGs. A finer analysis with different bins in magnitude confirms this tendency. Below $H p=8 \mathrm{mag}$, OBSG stars are about twice as often above $T_{\mathrm{h}}$ than below $\left(H p<6: 11 \%\right.$ below $T_{\mathrm{h}}, \approx 23 \%$ above $T_{\mathrm{h}} ; 6<H p<8$, $<18 \%$ below $T_{\mathrm{h}}, \approx 32 \%$ above $T_{\mathrm{h}}$ ). This would tend to indicate that OBSG stars are globally more variable than OB stars in general. Fainter than $H p=8 \mathrm{mag}$, on the other hand, there is no real difference between the percentage of OBSGs above and below $T_{\mathrm{h}}$ (OBSGs $\approx 25 \%$ of OB above and below $T_{\mathrm{h}}$, cf. Table 8 ) but this is probably due to the incompleteness of the sample, thus the increased scatter of the instrumental error fainter than $H p=8$ mag.

We also studied the distribution of periods in OBSGs to see if the trends seen in de Jager (1980, p.343) appear with our much larger sample of OBSG stars. As can be seen in Fig. 10, there is a tendency for longer periods $(20 \mathrm{~d}<P<70 \mathrm{~d})$ to be located near the upper right corner of the diagram, while shorter periods (5d $<P<10 \mathrm{~d})$ are located nearer to the lower left corner of 
Table 6. OBe stars above the threshold $T_{\mathrm{h}}$.

\begin{tabular}{|c|c|c|c|c|c|c|c|c|c|c|}
\hline $\begin{array}{c}\text { Var. } \\
\text { name }\end{array}$ & $\begin{array}{l}\mathrm{HD} \\
N_{\mathrm{o}} .\end{array}$ & $\begin{array}{c}\mathrm{HIP} \\
N_{\mathrm{o}} .\end{array}$ & $\begin{array}{l}\text { Var. } \\
\text { flag }\end{array}$ & $\begin{array}{l}\text { Var. } \\
\text { type }\end{array}$ & $\begin{array}{l}\text { HIP } \\
P(\mathrm{~d})\end{array}$ & $\begin{array}{l}\text { New } \\
P(\mathrm{~d})\end{array}$ & $\begin{array}{l}\text { CK } \\
P(\mathrm{~d})\end{array}$ & $\begin{array}{c}\langle H p\rangle \\
(\mathrm{mag})\end{array}$ & $\begin{array}{l}\text { Ampl. } \\
\text { (mag) }\end{array}$ & $\begin{array}{l}\text { Sp. } \\
\text { type }\end{array}$ \\
\hline \multirow[t]{2}{*}{ V1382 Ori } & 39680 & 27941 & $\bar{U}$ & $\mathrm{~L}$ & & & & 7.901 & 0.131 & O6:pe SB \\
\hline & 0 & 101425 & D & IA & & & & 8.851 & 0.088 & O6e \\
\hline NSV 14069 & 210839 & 109556 & U & IA & & & & 5.128 & 0.036 & O6e \\
\hline BN Gem & 60848 & 37074 & $\mathrm{U}$ & GCAS? & & 2.983 & & 6.868 & 0.054 & O8V:pevar \\
\hline \multirow[t]{2}{*}{ QZ Sge } & 188001 & 97796 & $\mathrm{U}$ & IA & & & & 6.232 & 0.047 & O8e \\
\hline & 192639 & 99768 & M & IA & & & & 7.197 & 0.048 & O8e \\
\hline \multirow[t]{2}{*}{ PZ Gem } & 45314 & 30722 & $\mathrm{U}$ & IA & & & & 6.617 & 0.043 & O9:pe \\
\hline & 24534 & 18350 & & IA & & & & 6.825 & 0.044 & O9.5pe \\
\hline V783 Cas & 0 & 10147 & U & IA? & & 6.330 & 36.900 & 9.910 & 0.182 & Bpe \\
\hline V725 Tau & 245770 & 26566 & $\mathrm{U}$ & $\mathrm{XNG/L}$ & & & & 9.267 & 0.256 & Bpe \\
\hline
\end{tabular}

Table 6 is presented in its entirety in Appendix A.

Table 7. OBSG stars above the threshold $T_{\mathrm{h}}$.

\begin{tabular}{|c|c|c|c|c|c|c|c|c|c|c|c|}
\hline $\begin{array}{c}\text { Var. } \\
\text { name }\end{array}$ & $\begin{array}{l}\mathrm{HD} \\
N_{\mathrm{o}} .\end{array}$ & $\begin{array}{l}\mathrm{HIP} \\
N_{\mathrm{o}} .\end{array}$ & $\begin{array}{l}\text { Var. } \\
\text { flag }\end{array}$ & $\begin{array}{l}\text { Var. } \\
\text { type }\end{array}$ & $\begin{array}{l}\text { HIP } \\
P(\mathrm{~d})\end{array}$ & $\begin{array}{l}\text { New } \\
P(\mathrm{~d}) \\
\end{array}$ & & $\begin{array}{c}\mathrm{CK} \\
P(\mathrm{~d}) \\
\end{array}$ & $\begin{array}{c}\langle H p\rangle \\
(\mathrm{mag})\end{array}$ & $\begin{array}{l}\text { Ampl. } \\
\text { (mag) }\end{array}$ & $\begin{array}{l}\text { Sp. } \\
\text { type }\end{array}$ \\
\hline & 66811 & 39429 & $\mathrm{M}$ & IA? & & 1.880 & & & 2.136 & 0.026 & O5Iaf \\
\hline & 206267 & 106886 & $\mathrm{U}$ & GCAS & & & & & 5.644 & 0.049 & O6 (f) \\
\hline \multirow[t]{3}{*}{ NSV 08031} & 152408 & 82775 & $\mathrm{U}$ & IA & & & & & 5.829 & 0.040 & $\mathrm{O} 8 \mathrm{Iab}+\ldots$ \\
\hline & 61827 & 37334 & $\mathrm{U}$ & IA & & & & & 7.749 & 0.093 & O8/O9Ib: \\
\hline & 152424 & 82783 & $\mathrm{U}$ & IA & & & & & 6.384 & 0.051 & O9Ia \\
\hline NSV 06024 & 112244 & 63117 & $\mathrm{U}$ & ACYG? & & 1.845 & & 2.003 & 5.372 & 0.045 & O9Ib \\
\hline \multirow[t]{4}{*}{ UV Aur } & 34842 & 25050 & $\mathrm{P}$ & M? & 394.420 & & & & 9.608 & 2.248 & O9II \\
\hline & 30614 & 22783 & $\mathrm{U}$ & L/IA? & & 1.776 & 0.885 & 0.885 & 4.295 & 0.040 & O9.5Ia SB: \\
\hline & 188209 & 97757 & $\mathrm{U}$ & ACYG? & & 5.234 & & 5.233 & 5.605 & 0.051 & O9.5Ia \\
\hline & 195592 & 101186 & $\mathrm{U}$ & ACYG? & & 1.832 & 2.414 & 0.085 & 7.215 & 0.062 & O9.5Ia \\
\hline
\end{tabular}

Table 7 is presented in its entirety in Appendix A.

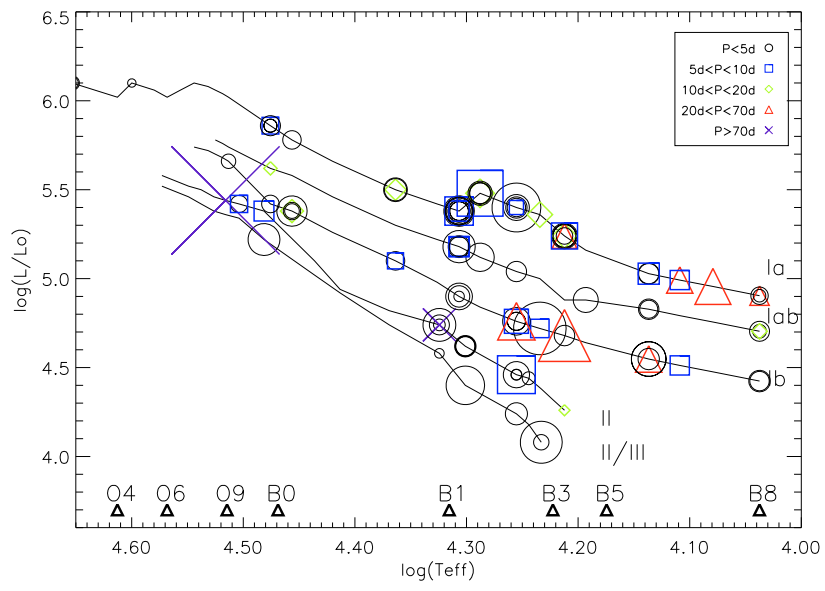

Fig. 10. Periodicities as a function of spectral type and luminosity class (cf. de Jager 1980). The tracks have been plotted according to the values of Vanbeveren et al. (1998). Symbol size is proportional to the amplitude of the variation.

the diagram. Intermediate periods $(10 \mathrm{~d}<P<20 \mathrm{~d})$ are mostly located between the above two groups. Short periods $(P<5 \mathrm{~d})$ are more scattered across the diagram (although still more to the lower left), but this tendency also appears when the new periods (some of them are not reliable) are not taken into account. Longer periods $(P>70 \mathrm{~d})$ are too few to conclude anything. Nevertheless, we confirm the effect shown in de Jager (1980) for a bigger sample with greater spread in spectral types. All the "variable" OBSG stars and their parameters are presented in Table 7.
Table 8. OBSG star proportions in 3 mag bins.

\begin{tabular}{cccccc}
\hline \hline & & $H_{\mathrm{p}} \leq 6$ & $6<H_{\mathrm{p}} \leq 8$ & $H_{\mathrm{p}}>8$ & Total \\
\hline OB stars & ampl. $\geq T_{\mathrm{h}}$ & 214 & 322 & 215 & 751 \\
& $T_{1} \leq$ ampl. $<T_{\mathrm{h}}$ & 90 & 270 & 291 & 651 \\
& ampl. $<T_{1}$ & 149 & 429 & 427 & 1005 \\
\hline Total & 453 & 1021 & 933 & 2407 \\
\hline OBSG stars & ampl. $\geq T_{\mathrm{h}}$ & 49 & 105 & 47 & 201 \\
& $T_{1} \leq$ ampl. $<T_{\mathrm{h}}$ & 14 & 65 & 98 & 177 \\
& ampl. $<T_{1}$ & 12 & 65 & 121 & 198 \\
\hline Total & 75 & 235 & 266 & 576 \\
\hline
\end{tabular}

Figure 8 also shows that there is a tendency for OBSG systems to have longer periods. This might be attributed in part to the larger size of the components (Söderhjelm \& Dischler 2005).

Finally, we note that the number of detached (EA) versus contact (EB) binary systems in this category is about equal, compared to a higher fraction of contact binaries among OBMS stars. Considering that surviving supergiant systems would have to have been farther apart than those that merged (Podsiadlowski 2006), this explains a greater relative number of detached systems among OBSGs.

\subsection{Special cases}

All the previously known periodically varying stars that were deleted because of a significant number of flagged points or were located below $T_{\mathrm{h}}$, are presented in Table 9 and their lightcurves are presented in Appendix B3 and B4. As indicated by their number $(\approx 1 \%)$, their effect on the threshold calculations is negligible. Moreover, the distribution of their periodicities and amplitudes does not visibly differ from the above results, and the 
Table 9. Periodic stars not included because of too many flagged points or below $T_{\mathrm{h}}$.

\begin{tabular}{|c|c|c|c|c|c|c|c|c|}
\hline $\begin{array}{r}\text { Var. } \\
\text { name }\end{array}$ & $\begin{array}{l}\mathrm{HD} \\
N_{\mathrm{o}} .\end{array}$ & $\begin{array}{l}\mathrm{HIP} \\
N_{\mathrm{o}} . \\
\end{array}$ & $\begin{array}{l}\text { Var. } \\
\text { flag }\end{array}$ & $\begin{array}{l}\text { Var. } \\
\text { type }\end{array}$ & $\begin{array}{l}\text { HIP } \\
P(\mathrm{~d}) \\
\end{array}$ & $\begin{array}{c}\left\langle H_{\mathrm{p}}\right\rangle \\
\text { (mag) } \\
\end{array}$ & $\begin{array}{l}\text { Ampl. } \\
\text { (mag) }\end{array}$ & $\begin{array}{l}\text { Sp. } \\
\text { type }\end{array}$ \\
\hline & & & & Not incl. & $\overline{\text { Total : } 7}$ & & & \\
\hline & & & & OBMS stars & Total : 6 & & & \\
\hline DH Cep & 215835 & 112470 & $\bar{P}$ & $?$ & 2.111 & 8.667 & 0.090 & O5.5 \\
\hline V1331 Aql & 173198 & 91910 & $\mathrm{P}$ & EA & 1.364 & 7.870 & 0.259 & B1Vvar \\
\hline V427 Cep & 239676 & 105960 & $\mathrm{P}$ & EB & 1.911 & 9.223 & 0.117 & B2 \\
\hline AN Dor & 31407 & 22663 & $\mathrm{P}$ & EA & 2.033 & 7.621 & 0.136 & B2/B3V \\
\hline HY Vel & 74560 & 42726 & $\mathrm{P}$ & $\mathrm{EB}$ ? & 3.102 & 4.773 & 0.055 & B3IV \\
\hline \multirow{2}{*}{ V686 CrA } & 175362 & 92989 & $\mathrm{P}$ & SPB? & 3.674 & 5.315 & 0.078 & B3V \\
\hline & & & & OBSG stars & Total : 1 & & & \\
\hline \multirow[t]{3}{*}{ V731 Mon } & 47240 & 31697 & $\bar{P}$ & $\overline{A C Y G}$ & 2.742 & 6.204 & 0.044 & B1Ib \\
\hline & & & & $\overline{\bar{B}}$ Below $T h$ & $\overline{\text { Total : } 18}$ & & & \\
\hline & & & & OBMS stars & Total : 14 & & & \\
\hline V486 Cas & 3950 & 3346 & $\overline{\mathrm{P}}$ & $?$ & 5.543 & 6.967 & 0.034 & $\overline{\text { B1III }}$ \\
\hline kap Sco & 160578 & 86670 & $\mathrm{P}$ & BCEP? & 0.202 & 2.317 & 0.019 & B1.5III \\
\hline V1046 Ori & 37017 & 26233 & $\mathrm{P}$ & SPB? & 0.901 & 6.518 & 0.029 & $\mathrm{~B} 1.5 \mathrm{~V}$ \\
\hline V470 Cyg & 228911 & 100193 & $\mathrm{P}$ & EB & 1.873 & 8.550 & 0.055 & $\mathrm{~B} 2+\ldots$ \\
\hline gam Peg & 886 & 1067 & $\mathrm{P}$ & ВСЕР & 0.152 & 2.755 & 0.022 & B2IV \\
\hline nu. Cen & 120307 & 67464 & $\mathrm{P}$ & SPB & 2.625 & 3.323 & 0.019 & B2IV \\
\hline V387 Cep & 217943 & 113853 & $\mathrm{P}$ & SPB & 4.176 & 6.738 & 0.034 & B2V \\
\hline V1377 Ori & 37055 & 26263 & $\mathrm{P}$ & SPB & 1.014 & 6.362 & 0.033 & B3IV \\
\hline KT Lup & 138769 & 76371 & $\mathrm{P}$ & SPB & 2.089 & 4.490 & 0.023 & B3IVp \\
\hline V490 Per & 25799 & 19178 & $\mathrm{P}$ & SPB & 0.912 & 7.065 & 0.038 & B3V... \\
\hline V1148 Tau & 29376 & 21575 & $\mathrm{P}$ & SPB & 1.061 & 6.993 & 0.041 & B3V \\
\hline XZ Lep & 41814 & 28973 & $\mathrm{P}$ & SPB & 0.935 & 6.608 & 0.029 & B3V \\
\hline IS Lup & 128585 & 71666 & $\mathrm{P}$ & SPB? & 0.855 & 9.312 & 0.091 & B3V \\
\hline \multirow[t]{2}{*}{ V2111 Cyg } & 191811 & 99415 & $\mathrm{P}$ & SPB & 1.442 & 7.637 & 0.052 & B3V \\
\hline & & & & OBe stars & Total : 1 & & & \\
\hline \multirow[t]{2}{*}{ V1012 Cen } & 128588 & 71709 & $\mathrm{P}$ & SPB? & 5.516 & 9.126 & 0.092 & B3Vne \\
\hline & & & & OBSG stars & Total : 3 & & & \\
\hline$\overline{\text { V2371 Oph }}$ & 157485 & 85189 & $\bar{P}$ & BCEP & 0.213 & 9.149 & 0.087 & $\overline{\mathrm{B} 1 / \mathrm{B} 2 \mathrm{Ib}}$ \\
\hline V348 Nor & 147985 & 80563 & $\mathrm{P}$ & ВСЕР & 0.132 & 7.964 & 0.060 & B1/B2II/III \\
\hline V856 Cen & 112481 & 63250 & $\mathrm{P}$ & BCEP & 0.255 & 8.373 & 0.070 & $\mathrm{~B} 2 \mathrm{Ib}$ \\
\hline
\end{tabular}

4 periodic OBSG stars that were deleted or located below the threshold $T_{\mathrm{h}}$ have periods in the range $P<5$ days and would not influence the interpretation of Fig. 10.

As mentioned earlier, LBV stars HR Car, AG Car and P Cyg (B2evar, B2:pe and B2pe, respectively) have been included in the OBe category. They represent $1 \%$ of the OBe stars and considering the error of $4 \%$ (cf. Table 2) on the level of variability of this class, it is not significant. Moreover, their HIPPARCOS data do not show any periodic variations and thus do not influence the results of the analysis of the periodic components. There are also a few unconfirmed LBV candidates according to Clark et al. (2005) within our sample but we cannot exclude them from this analysis without further evidence of their true status.

Star HIP34646 (HD55173, B3/5V(p)) was flagged under category 2 (corresponding to FAST data only) part of the time, hence the origin of the scatter. The unflagged points correspond to the "seemingly perfect" eclipsing binary lightcurve. However the value of the peak-to-peak amplitude indicated in the table in Appendix A represents the real amplitude of the variations due to binarity because the errors (significantly larger on the flagged points in this case) were taken into account.

Stars HIP65474 and HIP81305 have been classified as binaries (with "?") because of the shape of the dips seen in the lightcurves and the position of the lack of points about 0.5 phase later than the "first" dip. Of course, our choice can be discussed as stressed by "?" added to their variability type, but in this case, those two stars do not affect the statistics.
Star HIP10486 is definitely variable, almost certainly periodic but we do not have the definitive period. It might be an eclipsing binary but changing the period might affect the shape of our light curve to a large extent. Stars HIP31593 and HIP78526 belong to the eclipsing binary group as shown in Table 4. They have also been represented in the "New periodic variables" group because they were not detected as variable before this study (HIP31593) or before the article by Otero et al. (2003, HIP78526).

Some Be stars show typical outbursts (e.g. HIP32947, HIP35933, HIP74147, HIP88149). There are 3 studied Oe stars in the sample (HIP27941, HIP37074 and HIP30722) and their spectral types have been estimated to be too early (Negueruela et al. 2004), they are rather O8.5Ve, B0IVe and O9.5IVe, respectively. Rauw et al. (2007) find long term spectroscopic variations for HIP30722 (HD45314) and HIP37074 (HD60848), and our study points toward a new shorter period of 2.983 days for HIP37074, that remains to be studied in more detail.

\section{Conclusions}

First of all, we note that although this analysis is presented for $H p \leq 10 \mathrm{mag}$, the sample is complete only down to $H p=$ $8 \mathrm{mag}$. All the figures and statistics have also been calculated when limiting the sample to $H p=8 \mathrm{mag}$, and no major difference was noted. Thus the conclusions are not affected by our choice of limiting magnitude. 
In our sample, there are about $26 \pm 2 \%$ intrinsically variable stars, which compares to the statistics given in Marchenko et al. (1998) for the WR-stars of $\approx 33 \pm 19 \%$. These results are similar, with a possibly higher variability among WR stars probably induced by the presence of the dense stellar winds (e.g., rotational modulation: WR6, dust formation episodes or blobs: WR137).

This analysis has enabled us to constrain the differences of variability among three classes of intrinsic variability (OBMS, $\mathrm{OBe}$ and OBSG stars). Indeed, OBe stars are much more variable than the two other intrinsic classes (75\% compared to 17 and $35 \%$ for OBMS ans OBSG stars, respectively). The large physical sizes of the OBSG stars may explain the longer periods, compared to the OBMS sample, of the eclipsing binaries with an OBSG component. On the other hand, we note that there are more eclipsing binaries among OBMS stars, probably because those stars are more stable. Moreover, the proportion of contact systems is twice that of detached systems among OBMS stars. This might be an observational bias related to the closeness of the components in OBMS systems (i.e., contact systems are more variable and thus easier to observe).

The unique photometric data base of the HIPPARCOS program archive has enabled us to find a number of useful trends in variability among OB stars. It is expected that large, uniform photometric programs, space or ground-based, will provide even more impressive quantities of data of even higher quality. It is already the case for space missions such as the Canadian satellite MOST, the European COROT and the newly launched KEPLER mission. Large quantities of extremely precise data will soon enable one to enlarge this kind of statistical analysis of the variability of OB stars on other timescales.
Acknowledgements. We would like to thank the referee for very useful comments on this work. LL's work is supported by the CNES. A.F.J.M. is grateful for financial support from NSERC (Canada) and FQRNT (Québec).

\section{References}

Clark, J. S., Larionov, V. M., \& Arkharov, A. 2005, A\&A, 435, 239

Conti, P. S., \& Leep, E. M. 1974, ApJ, 193, 113

de Jager, C. 1980, Geophys. Astrophys. Monographs, 19

Duquennoy, A., Mayor, M., \& Halbwachs, J.-L. 1991, A\&AS, 88, 281

ESA 1997, VizieR Online Data Catalog, 1

Gautschy, A., \& Saio, H. 1996, ARA\&A, 34, 551

Huat, A.-L. 2009, A\&A, 0, 0

Koen, C., \& Eyer, L. 2002, MNRAS, 331, 45

Mann, H., \& Whitney, D. 1947, Annals Math. Stat., 18, 50

Marchenko, S. V., Moffat, A. F. J., van der Hucht, K. A., et al. 1998, A\&A, 331, 1022

Negueruela, I., Steele, I. A., \& Bernabeu, G. 2004, Astron. Nachr., 325, 749

Otero, S. A. 2003, Informational Bulletin on Variable Stars, 5480, 1

Podsiadlowski, P. 2006, in Sacacomie Workshop: Massive Stars in Interacting Binaries

Pols, O. R., Cote, J., Waters, L. B. F. M., \& Heise, J. 1991, A\&A, 241, 419

Quirrenbach, A., Bjorkman, K. S., Bjorkman, J. E., et al. 1997, ApJ, 479, 477

Rauw, G., Naze, Y., Marique, P. X., et al. 2007, Information Bulletin on Variable Stars, 5773, 1

Samus, N. N., Durlevich, O. V., et al. 2004, VizieR Online Data Catalog, 2250, 0

Söderhjelm, S., \& Dischler, J. 2005, A\&A, 442, 1003

Vanbeveren, D., van Rensbergen, W., \& de Loore, C. 1998, The brightest binaries, ed. D. Vanbeveren, W. van Rensbergen, \& C. De Loore (Boston: Kluwer Academic), Ap\&SS library, 232

Walborn, N. R., \& Fitzpatrick, E. L. 1990, PASP, 102, 379

Wilcoxon, F. 1945, Biometrics Bull., 1, 80 


\section{Appendix A: Variable OB stars in the HIPPARCOS catalog according to this study}

This appendix presents tables of the stars classified as "variable" in this study. The variability types used here are described in the "general catalog of variable stars" (Samus et al. 2004) and in Gautschy \& Saio (1996). The main types are I, IA, L, SDOR et GCAS for irregular variations; ACYG, BCEP, SPB, ACV, PVTEL, SR, SXARI for pulsating stars; and E, EA, EB for eclipsing binaries.

Table A.1. OB eclipsing binaries of the HIPPARCOS catalog.

\begin{tabular}{|c|c|c|c|c|c|c|c|c|c|c|c|}
\hline $\begin{array}{r}\text { Var. } \\
\text { name }\end{array}$ & $\begin{array}{l}\mathrm{HD} \\
N_{\mathrm{o}} .\end{array}$ & $\begin{array}{l}\mathrm{HIP} \\
N_{\mathrm{o}} .\end{array}$ & $\begin{array}{l}\text { Var. } \\
\text { flag }\end{array}$ & $\begin{array}{l}\text { Var. } \\
\text { type }\end{array}$ & $\begin{array}{l}\text { HIP } \\
P(\mathrm{~d}) \\
\end{array}$ & $\begin{array}{l}\text { New } \\
P(\mathrm{~d})\end{array}$ & & $\begin{array}{c}\text { CK } \\
P(\mathrm{~d})\end{array}$ & $\begin{array}{c}\left\langle H_{\mathrm{p}}\right\rangle \\
(\mathrm{mag})\end{array}$ & $\begin{array}{l}\text { Ampl. } \\
\text { (mag) }\end{array}$ & $\begin{array}{l}\text { Sp. } \\
\text { type }\end{array}$ \\
\hline & & & & & OBMS & & Total : 94 & & & & \\
\hline V444 Cyg & 193576 & 100214 & $P$ & EA & 4.213 & & & & 8.060 & 0.243 & O6 \\
\hline NX Vel & 73882 & 42433 & $\mathrm{P}$ & E: & 1.460 & & & & 7.292 & 0.074 & O8V: \\
\hline V1182 Aql & 175514 & 92865 & $\mathrm{P}$ & EB & 1.622 & & & & 8.747 & 0.191 & O8:Vnn \\
\hline TU Mus & 100213 & 56196 & $\mathrm{P}$ & $\mathrm{EB} / \mathrm{KE}$ & 1.387 & & & & 8.427 & 0.518 & $\mathrm{O} 8(+\mathrm{O} 8)$ \\
\hline V382 Cyg & 228854 & 100135 & $P$ & EB & 1.886 & & & & 8.730 & 0.830 & O8 \\
\hline del Cir & 135240 & 74778 & $\mathrm{P}$ & EA & 3.902 & & & & 5.056 & 0.154 & $08.5 \mathrm{~V}$ \\
\hline $\mathrm{AO}$ Cas & 1337 & 1415 & $\mathrm{P}$ & EB & 3.523 & & & & 6.062 & 0.198 & O9IIInn \\
\hline CC Cas & 19820 & 15063 & $\mathrm{P}$ & EB/DM & 3.366 & & & & 7.237 & 0.108 & O9IV \\
\hline LZ Cep & 209481 & 108772 & $\mathrm{P}$ & EB & 3.070 & & & & 5.554 & 0.099 & $\mathrm{O} 9 \mathrm{~V}$ \\
\hline LY Aur & 35921 & 25733 & $P$ & $\mathrm{~EB} / \mathrm{SD}$ & 4.003 & & & & 6.944 & 0.722 & O9.5III \\
\hline XZ Cep & & 111257 & $\mathrm{P}$ & EB/DM: & 5.098 & & & & 8.619 & 0.753 & $09.5 \mathrm{~V}$ \\
\hline V745 Cas & 1810 & 1805 & $\mathrm{P}$ & EW & 1.411 & & & & 8.104 & 0.142 & B0IV \\
\hline AB Cru & 106871 & 59935 & $\mathrm{P}$ & EB & 3.413 & & & & 8.527 & 0.567 & B0IVvar \\
\hline V2107 Cyg & 191473 & 99246 & $\mathrm{P}$ & EA & 4.285 & & & & 8.601 & 0.130 & B0IV \\
\hline Y Cyg & 198846 & 102999 & $P$ & EA/DM & 2.996 & & & & 7.335 & 0.473 & B0IVv SB \\
\hline NY Cep & 217312 & 113461 & $\mathrm{P}$ & EA & 15.276 & & & & 7.514 & 0.086 & B0IV \\
\hline V478 Cyg & 193611 & 100227 & $P$ & EB & 2.881 & & & & 8.799 & 0.261 & B $0 V p$ \\
\hline V649 Cas & 219634 & 114904 & $P$ & EA & 2.391 & & & & 6.598 & 0.069 & BoVn \\
\hline UU Cas & & 117576 & $\mathrm{P}$ & EB/DM & 8.520 & & & & 9.934 & 0.379 & B0.5III \\
\hline del Pic & 42933 & 29276 & $\mathrm{P}$ & EB/D: & 1.673 & & & & 4.651 & 0.205 & B0.5IV \\
\hline \multirow[t]{2}{*}{ AI Cep } & 239767 & 107500 & $P$ & EB/DM & 4.225 & & & & 9.424 & 0.577 & B0.5V:pvar \\
\hline & 213405 & $110998^{a}$ & $\mathrm{U}$ & EA? & & 2.847 & & 2.847 & 8.099 & 0.077 & B0.5V \\
\hline AH Cep & 216014 & 112562 & $\mathrm{P}$ & EW & 1.775 & & & & 6.977 & 0.277 & B $0.5 \mathrm{~V}: \mathrm{nn}$ \\
\hline V431 Pup & 69882 & 40596 & $\mathrm{P}$ & $\mathrm{E}$ & 9.363 & & & & 7.234 & 0.080 & B1III: \\
\hline VZ Cen & 103146 & 57895 & $\mathrm{P}$ & EB & 4.929 & & & & 8.350 & 0.352 & B1III:var \\
\hline HX Lup & 125721 & 70270 & $P$ & EB? & 3.088 & & & & 6.056 & 0.046 & B1III \\
\hline V380 Cyg & 187879 & 97634 & $\mathrm{P}$ & EA & 12.426 & & & & 5.676 & 0.101 & B1III \\
\hline V1898 Cyg & 200776 & 103968 & $\mathrm{P}$ & EB & 1.513 & & & & 7.812 & 0.241 & B1IV:p \\
\hline EM Cep & 208392 & 108073 & $\mathrm{P}$ & EW & 0.806 & & & & 7.085 & 0.126 & B1IV: \\
\hline eta Ori & 35411 & 25281 & $\mathrm{P}$ & EA/BCE & 7.990 & & & & 3.312 & 0.243 & $\mathrm{~B} 1 \mathrm{~V}+\mathrm{B} 2$ \\
\hline VV Ori & 36695 & 26063 & $\mathrm{P}$ & EB & 1.485 & & & & 5.327 & 0.257 & B1V \\
\hline V Pup & 65818 & 38957 & $\mathrm{P}$ & $\mathrm{EB} / \mathrm{SD}$ & 1.455 & & & & 4.433 & 0.533 & $\mathrm{~B} 1 \mathrm{Vp}+\mathrm{B} 2$ \\
\hline LN Mus & 110946 & 62339 & $P$ & EB & 3.672 & & & & 9.265 & 0.311 & B1V: \\
\hline alf Vir & 116658 & 65474 & $\mathrm{P}$ & EB? & 4.014 & & & & 0.888 & 0.035 & B1V \\
\hline eta Cen & 127972 & 71352 & $\mathrm{P}$ & EB & 1.285 & & & & 2.276 & 0.071 & $\mathrm{~B} 1 \mathrm{Vn}+\mathrm{A}$ \\
\hline pi. Sco & 143018 & 78265 & $\mathrm{P}$ & EB & 1.570 & & & & 2.829 & 0.037 & $\mathrm{~B} 1 \mathrm{~V}+\mathrm{B} 2 \mathrm{~V}$ \\
\hline V1012 Sco & 155775 & 84409 & $\mathrm{P}$ & EB & 1.515 & & & & 6.717 & 0.061 & B1V \\
\hline V701 Sco & 317844 & 85985 & $\mathrm{P}$ & EW/KE & 0.762 & & & & 8.811 & 0.454 & B1:V:nn \\
\hline V1331 Aql & 173198 & $91910^{d}$ & $\mathrm{P}$ & EA & 1.364 & & & & 7.870 & 0.259 & B1Vvar \\
\hline V446 Cep & 210478 & 109311 & $\mathrm{P}$ & EA & 3.808 & & & & 7.355 & 0.079 & B1V \\
\hline CW Cep & 218066 & 113907 & $\mathrm{P}$ & EA/DM & 2.729 & & & & 7.736 & 0.251 & B1:V:var \\
\hline mu.01 Sco & 151890 & 82514 & $\mathrm{P}$ & $\mathrm{EB} / \mathrm{SD}$ & 1.446 & & & & 2.957 & 0.273 & $\mathrm{~B} 1.5 \mathrm{IV}+\mathrm{B}$ \\
\hline V436 Per & 11241 & 8704 & $\mathrm{P}$ & EA/D & 25.936 & & & & 5.485 & 0.237 & B $1.5 \mathrm{~V}$ \\
\hline HX Vel & 74455 & 42712 & $\mathrm{P}$ & EB? & 1.124 & & & & 5.417 & 0.044 & $\mathrm{~B} 1.5 \mathrm{Vn}$ \\
\hline u Her & 156633 & 84573 & $P$ & EB & 2.051 & & & & 4.785 & 0.404 & $\mathrm{~B} 1.5 \mathrm{Vp}$ \\
\hline pi.05 Ori & 31237 & 22797 & $\mathrm{P}$ & EB? & 3.700 & & & & 3.641 & 0.058 & B2III SB \\
\hline MS CMa & 56554 & 35168 & $\mathrm{P}$ & EA & 6.171 & & & & 7.095 & 0.098 & B2III/IV \\
\hline psi Ori & 35715 & 25473 & $\mathrm{P}$ & EB & 2.526 & & & & 4.514 & 0.044 & B2IV \\
\hline V954 Sco & 149779 & 81508 & $\mathrm{P}$ & EB & 1.269 & & & & 7.625 & 0.273 & B2IV \\
\hline V461 Car & 66546 & 39225 & $\mathrm{P}$ & EA & 2.515 & & & & 6.119 & 0.067 & B2IV-V \\
\hline V1384 Ori & 40005 & 28142 & $P$ & EA & 6.572 & & & & 7.203 & 0.233 & $\mathrm{~B} 2 \mathrm{~V}$ \\
\hline V1388 Ori & 42401 & 29321 & $\mathrm{P}$ & EB & 2.187 & & & & 7.494 & 0.343 & $\mathrm{~B} 2 \mathrm{~V}$ \\
\hline LZ CMa & 54912 & 34579 & $\mathrm{P}$ & EB & 3.309 & & & & 5.635 & 0.044 & B2V \\
\hline MX CMa & 57192 & 35461 & $\mathrm{P}$ & EB & 2.486 & & & & 6.757 & 0.065 & B2V \\
\hline QZ Pup & 64503 & 38455 & $\mathrm{P}$ & EB & 1.112 & & & & 4.430 & 0.058 & B2V \\
\hline TU Mon & & 38523 & $\mathrm{P}$ & EB & 5.049 & & & & 9.286 & 0.183 & $\mathrm{~B} 2 \mathrm{Vn}$ \\
\hline XY Pyx & 71801 & 41515 & $\mathrm{P}$ & EB & 0.923 & & & & 5.702 & 0.069 & $\mathrm{~B} 2 \mathrm{~V}$ \\
\hline CV Vel & 77464 & 44245 & $\mathrm{P}$ & EA/DM & 6.889 & & & & 6.692 & 0.424 & $\mathrm{~B} 2 \mathrm{~V}+\mathrm{B} 2 \mathrm{~V}$ \\
\hline V1040 Sco & 142184 & $77859^{b}$ & $P$ & $\mathrm{~EB} / \mathrm{SD}$ & 0.508 & 0.508 & 1.017 & & 5.398 & 0.037 & B2V \\
\hline V539 Ara & 161783 & 87314 & $\mathrm{P}$ & EA/DM & 3.169 & & & & 5.699 & 0.372 & $\mathrm{~B} 2 \mathrm{~V}+\mathrm{B} 3 \mathrm{~V}$ \\
\hline V599 Aql & 176853 & 93502 & $\mathrm{P}$ & EB & 1.846 & & & & 6.707 & 0.044 & $\mathrm{~B} 2 \mathrm{~V}$ \\
\hline V1773 Cyg & 193536 & 100142 & $\mathrm{P}$ & $\mathrm{EB}$ ? & 2.985 & & & & 6.423 & 0.055 & $\mathrm{~B} 2 \mathrm{~V}$ \\
\hline V1792 Cyg & 198784 & 102953 & $\mathrm{P}$ & EB & 3.303 & & & & 7.308 & 0.100 & $\mathrm{~B} 2 \mathrm{~V}$ \\
\hline V383 Cep & 208106 & 107913 & $\mathrm{P}$ & EB & 1.496 & & & & 7.454 & 0.328 & B2Vnp \\
\hline V470 Cyg & 228911 & $100193^{d}$ & $\mathrm{P}$ & EB & 1.873 & & & & 8.550 & 0.055 & $\mathrm{~B} 2+\ldots$ \\
\hline V427 Cep & 239676 & $105960^{d}$ & $\mathrm{P}$ & EB & 1.911 & & & & 9.223 & 0.117 & B2 \\
\hline NO Vel & 69144 & 40285 & $\mathrm{P}$ & EB & 4.823 & & & & 5.090 & 0.035 & B2.5IV \\
\hline IT Lib & 138503 & 76161 & $\mathrm{P}$ & EB & 2.267 & & & & 9.111 & 0.522 & B2/3IV:n: \\
\hline AN Dor & 31407 & $22663^{d}$ & $\mathrm{P}$ & EA & 2.033 & & & & 7.621 & 0.136 & B2/B3V \\
\hline V4197 Sgr & 177559 & 93785 & $\mathrm{P}$ & EW & 0.715 & & & & 8.253 & 0.648 & $\mathrm{~B} 2 / 3 \mathrm{~V}(\mathrm{n})$ \\
\hline
\end{tabular}


Table A.1. continued.

\begin{tabular}{|c|c|c|c|c|c|c|c|c|c|c|c|}
\hline $\begin{array}{l}\text { Var. } \\
\text { name }\end{array}$ & $\begin{array}{l}\mathrm{HD} \\
N_{\mathrm{o}} .\end{array}$ & $\begin{array}{l}\mathrm{HIP} \\
N_{\mathrm{o}} .\end{array}$ & $\begin{array}{l}\text { Var. } \\
\text { flag }\end{array}$ & $\begin{array}{l}\text { Var. } \\
\text { type }\end{array}$ & $\begin{array}{l}\text { HIP } \\
P(\mathrm{~d})\end{array}$ & $\begin{array}{l}\text { New } \\
P(\mathrm{~d})\end{array}$ & & $\begin{array}{l}\text { CK } \\
P(\mathrm{~d})\end{array}$ & $\begin{array}{c}\left\langle H_{\mathrm{p}}\right\rangle \\
(\mathrm{mag})\end{array}$ & $\begin{array}{l}\text { Ampl. } \\
\text { (mag) }\end{array}$ & $\begin{array}{l}\text { Sp. } \\
\text { type }\end{array}$ \\
\hline V393 Sco & 161741 & 87191 & $\bar{P}$ & EB & 7.713 & & & & 7.613 & 0.674 & B3III \\
\hline HY Vel & 74560 & $42726^{d}$ & $\mathrm{P}$ & & 3.102 & & & & 4.773 & 0.055 & B3IV \\
\hline V360 Lac & 216200 & 112778 & $\mathrm{P}$ & & 10.082 & & & & 5.943 & 0.063 & B3IV:var \\
\hline AR Cas & 221253 & 115990 & $\mathrm{P}$ & & 6.066 & & & & 4.849 & 0.039 & B3IV \\
\hline lam Tau & 25204 & 18724 & $\mathrm{P}$ & & 3.953 & & & & 3.395 & 0.128 & $\mathrm{~B} 3 \mathrm{~V}+\mathrm{A}$ \\
\hline EO Aur & 34333 & 24744 & $P$ & EB/SD & 4.066 & & & & 7.871 & 0.541 & $\mathrm{~B} 3 \mathrm{~V}+\mathrm{B} 3 \mathrm{~V}$ \\
\hline WX Lep & 37622 & 26612 & $\mathrm{P}$ & EB & 1.840 & & & & 7.966 & 0.088 & B3Vn \\
\hline \multirow{2}{*}{ AE Pic } & 46792 & 31068 & $\mathrm{P}$ & EB & 2.982 & & & & 6.103 & 0.057 & B3V \\
\hline & 47247 & $31593^{b}$ & $\mathrm{U}$ & EA & & 0.993 & 1.986 & 2.095 & 6.315 & 0.093 & B3V \\
\hline V462 Car & 66768 & 39310 & $\mathrm{P}$ & EB/IA & 1.106 & & & & 6.724 & 0.166 & B3V(n) \\
\hline V438 Pup & 71302 & $41250^{c}$ & $\mathrm{U}$ & EA? & & 1.183 & & & 5.918 & 0.139 & B3V \\
\hline QX Car & 86118 & 48589 & $\mathrm{P}$ & EA/DM & 4.478 & & & & 6.626 & 0.395 & $\mathrm{~B} 3 \mathrm{~V}+\mathrm{B} 3 \mathrm{~V}$ \\
\hline V345 Vel & 90000 & 50780 & $\mathrm{P}$ & EA & 10.495 & & & & 7.528 & 0.211 & B3V \\
\hline V3894 Sgr & 161756 & 87163 & $\mathrm{P}$ & EB & 2.619 & & & & 6.352 & 0.130 & B3Vn \\
\hline V1441 Aql & 177624 & 93732 & $\mathrm{P}$ & EB & 2.374 & & & & 6.937 & 0.102 & B3V \\
\hline sig Aql & 185507 & 96665 & $\mathrm{P}$ & EB & 1.950 & & & & 5.195 & 0.125 & $\mathrm{~B} 3 \mathrm{~V}+\mathrm{B} 3 \mathrm{~V}$ \\
\hline MR Cyg & & 108508 & $\mathrm{P}$ & EB & 1.677 & & & & 8.881 & 0.524 & B3V \\
\hline GT Cep & 217224 & 113385 & $P$ & EA/SD & 4.909 & & & & 8.279 & 0.629 & B3V \\
\hline FZ CMa & 52942 & 33953 & $\mathrm{P}$ & EA & 1.273 & & & & 8.170 & 0.284 & B3n \\
\hline AO Mon & 53883 & 34299 & $\mathrm{P}$ & EA & 1.885 & & & & 9.676 & 0.547 & B3+B5 \\
\hline V2126 Cyg & 235271 & 101439 & $\mathrm{P}$ & EB & 5.891 & & & & 9.023 & 0.134 & B3 \\
\hline RS Sgr & 167647 & 89637 & $\mathrm{P}$ & EB & 2.416 & & & & 6.025 & 0.209 & B3/B4IV/V \\
\hline FF CMa & 55173 & 34646 & $\mathrm{P}$ & $\mathrm{EB} / \mathrm{KE}$ & 1.213 & & & & 7.468 & 0.444 & B3/5V(p) \\
\hline \multirow[t]{2}{*}{ zet Phe } & 6882 & 5348 & $\mathrm{P}$ & EA/DM & 1.670 & & & & 3.953 & 0.304 & $\mathrm{~B} 6 \mathrm{~V}+\mathrm{B} 0 \mathrm{~V}$ \\
\hline & & & & & $\mathrm{OBe}$ & & Total : 7 & & & & \\
\hline V1036 Sco & 159176 & 86011 & $\bar{P}$ & EB? & 3.367 & & & & 5.705 & 0.040 & O5/6(e) \\
\hline V1007 Sco & 152248 & 82691 & $\mathrm{P}$ & EA & 5.817 & & & & 6.125 & 0.127 & O7e \\
\hline V729 Cyg & & 101341 & $\mathrm{P}$ & $\mathrm{EB} / \mathrm{D} / \mathrm{G}$ & 6.597 & & & & 9.139 & 0.278 & O7e \\
\hline SX Aur & 33357 & 24201 & $\mathrm{P}$ & $\mathrm{EB} / \mathrm{KE}$ & 1.210 & & & & 8.518 & 0.482 & B1:V:ne: \\
\hline AI Cru & & 59026 & $\mathrm{P}$ & EB & 1.418 & & & & 9.645 & 0.631 & B2IVe \\
\hline GU CMa & 52721 & 33868 & $P$ & EB & 1.610 & & & & 6.579 & 0.214 & B2Vne \\
\hline \multirow[t]{2}{*}{ IU Aur } & 35652 & 25565 & $\mathrm{P}$ & $\mathrm{EB} / \mathrm{SD}$ & 1.811 & & & & 8.439 & 0.546 & B3Vnne \\
\hline & & & & & OBSG & & Total : 26 & & & & \\
\hline V884 Sco & 153919 & 83499 & $\mathrm{P}$ & EB & 3.411 & & & & 6.573 & 0.066 & $\mathrm{O} 5 \mathrm{f}$ \\
\hline UW CMa & 57060 & 35412 & $\mathrm{P}$ & EB/KE: & 4.393 & & & & 4.904 & 0.421 & O7f \\
\hline MY Ser & 167971 & 89681 & $\mathrm{P}$ & EB & 3.322 & & & & 7.549 & 0.237 & O8/9f \\
\hline V918 Sco & 149404 & 81305 & $\mathrm{P}$ & EA? & 9.813 & & & & 5.540 & 0.037 & O9Ia \\
\hline tau CMa & 57061 & 35415 & $P$ & EW/GS & 1.282 & & & & 4.336 & 0.058 & O9Ib \\
\hline V453 Sco & 163181 & 87810 & $\mathrm{P}$ & EB/GS & 12.006 & & & & 6.684 & 0.413 & O9.5Ia/ab \\
\hline del Ori & 36486 & 25930 & $\mathrm{P}$ & EA & 5.732 & & & & 2.148 & 0.097 & O9.5II \\
\hline QZ Car & 93206 & 52526 & $\mathrm{P}$ & EB/GS & 5.999 & & & & 6.325 & 0.206 & B0Ib: \\
\hline V861 Sco & 152667 & 82911 & $\mathrm{P}$ & EB/GS & 7.848 & & & & 6.260 & 0.260 & B0.5Ia \\
\hline GP Vel & 77581 & 44368 & $P$ & $\mathrm{E}$ & 8.965 & & & & 7.005 & 0.096 & B0.5Ib \\
\hline V1765 Cyg & 187459 & 97485 & $\mathrm{P}$ & EA/GS & 13.374 & & & & 6.499 & 0.100 & B0.5Ibvar \\
\hline V373 Cas & 224151 & 117957 & $\mathrm{P}$ & E & 13.419 & & & & 6.087 & 0.069 & B0.5IIv SB \\
\hline V421 Pup & 68026 & 39968 & $P$ & EB & 5.417 & & & & 9.093 & 0.145 & B1II \\
\hline QR Ser & 168183 & 89753 & $\mathrm{P}$ & EB: & 4.016 & & & & 8.313 & 0.112 & B1Ib/II \\
\hline V448 Cyg & 190967 & 99021 & $\mathrm{P}$ & EB & 6.520 & & & & 8.216 & 0.602 & B1Ib-II \\
\hline FY Vel & 72754 & 41882 & $\mathrm{P}$ & EB:/GS & 33.840 & & & & 6.944 & 0.264 & B2Iape \\
\hline V390 Pup & 62747 & 37751 & $\mathrm{P}$ & EA & 3.928 & & & & 5.556 & 0.059 & B2II \\
\hline V399 Pup & 64014 & 38186 & $\mathrm{P}$ & EA & 3.910 & & & & 9.343 & 0.222 & B2II \\
\hline FM CMa & 53756 & 34221 & $\mathrm{P}$ & EA & 2.789 & & & & 7.306 & 0.258 & B2/B3II \\
\hline V1069 Sco & 152504 & 82819 & $\mathrm{P}$ & EA & 5.347 & & & & 8.620 & 0.243 & B2/B3II \\
\hline V1081 Sco & 158186 & 85569 & $\mathrm{P}$ & EA & 8.770 & 8.770 & 1.957 & & 7.004 & 0.099 & B2/B3II \\
\hline RZ Sct & 169753 & 90382 & $P$ & EA/GS & 15.190 & & & & 7.675 & 0.314 & B3Ib \\
\hline V368 Cas & 19644 & 14936 & $\mathrm{P}$ & EA & 4.452 & & & & 8.365 & 0.438 & B3II-III \\
\hline V438 Per & 13970 & 10704 & $\mathrm{P}$ & EB/GS? & 3.509 & & & & 8.360 & 0.092 & B5Ib \\
\hline V505 Mon & 48914 & 32397 & $\mathrm{P}$ & EB/GS & 53.781 & 53.780 & 30.303 & & 7.231 & 0.249 & B5Ib \\
\hline MP TrA & 143028 & $78526^{b, c}$ & $\mathrm{U}$ & EA & & 2.070 & & $2.070^{c}$ & 7.789 & 0.099 & B7Ib/II \\
\hline
\end{tabular}

Table A.1 is the complete version of Table 4. The columns are: the variable name, the HD number, the HIPPARCOS number, the HIPPARCOS variability flag, the variability type we assigned them in this study, the HIPPARCOS period (if any), the new periods found in this study ( 2 columns, for a possible second period), the periods found by Koen \& Eyer (2002) (if any), the $H p$ magnitude, the mean amplitude calculated here and the spectral type according to the Vizier database.

${ }^{a}$ Koen et al. (2002); ${ }^{b}$ This study; ${ }^{c}$ Otero et al. (2003); ${ }^{d}$ refers to the stars that were not included in the study or below $T_{\mathrm{h}}$. 
Table A.2. OBMS stars above the threshold $T_{\mathrm{h}}$.

\begin{tabular}{|c|c|c|c|c|c|c|c|c|c|c|}
\hline $\begin{array}{r}\text { Var. } \\
\text { name }\end{array}$ & $\begin{array}{l}\mathrm{HD} \\
N_{\mathrm{o}} .\end{array}$ & $\begin{array}{l}\mathrm{HIP} \\
N_{\mathrm{o}} .\end{array}$ & $\begin{array}{l}\text { Var. } \\
\text { flag }\end{array}$ & $\begin{array}{l}\text { Var. } \\
\text { type }\end{array}$ & $\begin{array}{l}\text { HIP } \\
P(\mathrm{~d})\end{array}$ & $\begin{array}{l}\text { New } \\
P(\text { d) }\end{array}$ & $\begin{array}{l}\text { CK } \\
P(\mathrm{~d}) \\
\end{array}$ & $\begin{array}{c}\left\langle H_{\mathrm{p}}\right\rangle \\
(\mathrm{mag})\end{array}$ & $\begin{array}{l}\begin{array}{l}\text { Ampl. } \\
\text { (mag) }\end{array} \\
\end{array}$ & $\begin{array}{l}\begin{array}{l}\text { Sp. } \\
\text { type }\end{array} \\
\end{array}$ \\
\hline & 97950 & 54948 & $\mathrm{D}$ & IA & & & & 9.019 & 0.114 & O5 \\
\hline \multirow[t]{3}{*}{ S Mon } & 47839 & 31978 & U & SR/L? & & 3.271 & & 4.551 & 0.040 & O7 \\
\hline & 91824 & 51773 & & IA & & & & 8.129 & 0.068 & $\mathrm{O} 7$ \\
\hline & 152623 & 82876 & D & IA & & & & 6.726 & 0.043 & $\mathrm{O} 7$ \\
\hline \multirow{3}{*}{$\begin{array}{l}\text { NSV } 08060 \\
\text { gam02 Vel }\end{array}$} & 152723 & 82936 & $\mathrm{U}$ & BCEP? & & 0.395 & & 7.150 & 0.065 & $\mathrm{O} 7$ \\
\hline & & 39953 & $\mathrm{U}$ & BCEP? & & 1.384 & & 1.705 & 0.044 & O8III \\
\hline & 96670 & 54358 & $\mathrm{U}$ & IA & & & & 7.467 & 0.055 & $\mathrm{O} 8$ \\
\hline V871 Cen & 101205 & 56769 & $\mathrm{U}$ & IA & & & & 6.474 & 0.092 & O8var \\
\hline & 124314 & 69628 & D & IA & & & & 6.696 & 0.040 & 08 \\
\hline V1809 Cyg & 203064 & 105186 & $\mathrm{U}$ & L? & & & & 5.022 & 0.031 & O8 \\
\hline & 57236 & 35493 & U & IA & & & & 8.799 & 0.128 & $\mathrm{O} 9 \mathrm{~V}$ \\
\hline V1075 Sco & 155806 & 84401 & $\mathrm{U}$ & IA & & & & 5.601 & 0.080 & O9 \\
\hline & 16429 & 12495 & D & IA? & & 12.053 & & 7.817 & 0.059 & O9.5III \\
\hline & 151564 & 82378 & $\mathrm{U}$ & IA & & & & 8.026 & 0.072 & O9.5IV \\
\hline AE Aur & 34078 & 24575 & $\mathrm{U}$ & IA? & & & & 6.053 & 0.056 & O9.5Vvar \\
\hline V961 Cen & 115071 & 64737 & $\mathrm{P}$ & SPB & 1.366 & & & 8.022 & 0.110 & $09.5 \mathrm{~V}$ \\
\hline & 125206 & 70052 & $\mathrm{U}$ & IA & & & & 7.981 & 0.072 & $09.5 \mathrm{~V}$ \\
\hline & 149757 & 81377 & M & IA & & & & 2.570 & 0.027 & $09.5 \mathrm{~V}$ \\
\hline V744 Her & & 87280 & $\mathrm{U}$ & ? & & 27.778 & & 6.746 & 0.042 & Bpsh \\
\hline V473 Per & 13831 & 10615 & $\mathrm{U}$ & IA & & & & 8.308 & 0.078 & B0IIIp \\
\hline NSV 01458 & 25638 & 19272 & U & IA & & & 2.871 & 7.042 & 0.630 & B0III \\
\hline DL Cam & 28446 & 21148 & U & IA & & & & 5.826 & 0.035 & B0III SB \\
\hline & 164340 & 88352 & U & IA & & & & 9.246 & 0.109 & B0III \\
\hline V808 Cas & & 114815 & $\mathrm{P}$ & SPB & 1.300 & & & 9.812 & 0.187 & B0III:p: \\
\hline RX Cyg & 192035 & 99424 & $\mathrm{U}$ & L? & & & & 8.242 & 0.075 & B0III-IV(n) \\
\hline & 81370 & 46032 & $\mathrm{U}$ & IA & & & & 8.796 & 0.096 & B0IV: \\
\hline V413 Lac & & 111071 & $\mathrm{P}$ & SPB & 1.509 & & & 9.786 & 0.144 & B0IVn \\
\hline SZ Cam & 25639 & 19270 & $\mathrm{P}$ & ? & 2.699 & & & 7.144 & 0.590 & B0V \\
\hline & 42259 & 29201 & $\mathrm{U}$ & IA & & & & 8.618 & 0.085 & BOV \\
\hline & 71609 & 41388 & $\mathrm{U}$ & IA & & & & 7.833 & 0.088 & BOV \\
\hline & & 106884 & D & $?$ & & & & 6.673 & 2.340 & BOV \\
\hline & & 106890 & $\mathrm{U}$ & $?$ & & & & 5.696 & 0.221 & B0V \\
\hline & 235549 & 106529 & & IA & & & & 9.086 & 0.097 & B0 \\
\hline & 22253 & 16917 & M & ? & & 0.762 & 0.762 & 6.596 & 0.038 & B0.5III \\
\hline $\mathrm{MN} \mathrm{CMa}$ & 55885 & 34986 & $\mathrm{U}$ & IA & & & & 9.631 & 0.170 & B $0.5 \mathrm{III}$ \\
\hline DF Cru & 104705 & 58783 & $\mathrm{P}$ & SPB & 1.135 & & & 7.813 & 0.074 & B0.5III \\
\hline bet Cru & 111123 & 62434 & $\mathrm{P}$ & BCEP & 0.191 & & & 1.156 & 0.028 & B0.5III \\
\hline & 119159 & 66925 & M & SPB? & & 1.103 & 0.901 & 5.976 & 0.034 & B0.5III \\
\hline & 192445 & 99667 & U & $\mathrm{L}$ & & & & 7.074 & 0.083 & B0.5III \\
\hline SY Equ & 203664 & 105614 & $\mathrm{P}$ & BCEP & 0.166 & & & 8.497 & 0.084 & B0.5IIIn \\
\hline & 191531 & 99327 & $\mathrm{U}$ & BCEP? & & 0.164 & 0.164 & 8.341 & 0.080 & B0.5III-IV \\
\hline V803 Cas & 237090 & 15139 & $\mathrm{U}$ & IA? & & 4.652 & 4.257 & 9.198 & 0.156 & B0.5IV:nn \\
\hline & 53755 & 34234 & M & BCEP? & & 0.561 & & 6.480 & 0.041 & B0.5IVn \\
\hline FN CMa & 53974 & 34301 & U & $\mathrm{BCEP}^{a}$ & & 1.529 & & 5.412 & 0.031 & B0.5IV \\
\hline & 126827 & 60718 & D & $\mathrm{L}$ & & & & 0.675 & 0.024 & B0.5IV \\
\hline CY Cru & & 62937 & $\mathrm{U}$ & ? & & & & 8.981 & 1.646 & B0.5IVn \\
\hline ES Vul & 180968 & 94827 & $\mathrm{U}$ & $\mathrm{BCEP}^{b}$ & & & & 5.458 & 0.037 & B0.5IV \\
\hline V1294 Aql & 184279 & 96196 & $\mathrm{U}$ & SPB? & & 7.752 & & 7.010 & 0.260 & B0.5IV \\
\hline V732 Mon & 47360 & 31739 & $\mathrm{P}$ & $?$ & 7.207 & & & 8.204 & 0.134 & B $0.5 \mathrm{~V}$ \\
\hline omi Per & 23180 & 17448 & $P$ & ELL? & 4.419 & & & 3.860 & 0.040 & B1III \\
\hline CY Cam & 24094 & 18151 & $\mathrm{P}$ & BCEP & 0.526 & & & 8.459 & 0.105 & B1III \\
\hline ksi01 CMa & 46328 & 31125 & $\mathrm{P}$ & BCEP & 0.210 & & & 4.256 & 0.050 & B1III \\
\hline & 90313 & 50919 & $\mathrm{U}$ & IA & & & & 8.443 & 0.089 & B1III \\
\hline bet Cen & 122451 & 68702 & $\mathrm{U}$ & $\mathrm{BCEP}^{c}$ & & & & 0.536 & 0.029 & B1III \\
\hline & 144969 & 79279 & $\mathrm{U}$ & SPB? & & 2.101 & & 8.408 & 0.074 & B1III \\
\hline sig Sco & 147165 & 80112 & $\mathrm{P}$ & BCEP & 0.247 & & & 2.923 & 0.056 & B1III \\
\hline & 3191 & 2816 & U & IA & & & & 8.687 & 0.082 & B1IV:nn \\
\hline V351 Per & 13051 & 10055 & $\mathrm{U}$ & $\mathrm{BCEP}^{d}$ & & & & 8.688 & 0.141 & B1IV: \\
\hline V455 Sct & 173637 & 92128 & $\mathrm{U}$ & $\mathrm{L}$ & & & & 9.225 & 0.252 & B1IV \\
\hline & 7252 & 5768 & $\mathrm{U}$ & BCEP? & & 0.872 & 0.872 & 7.196 & 0.049 & B1V SB \\
\hline V792 Cas & 17114 & 13016 & $\mathrm{P}$ & ELL? & 2.581 & & & 9.354 & 0.220 & B1V \\
\hline & & 29127 & D & IA? & & & & 8.528 & 0.265 & B1V \\
\hline & 43907 & 30075 & U & IA & & & & 8.684 & 0.113 & B1V:p: \\
\hline V746 Mon & 51193 & 33361 & $\mathrm{U}$ & IA? & & & & 8.081 & 0.099 & B1V:nn \\
\hline V637 Mon & 52918 & 33971 & $\mathrm{P}$ & BCEP & 0.191 & & & 4.919 & 0.061 & B1V \\
\hline QZ Vel & 85871 & 48469 & $\mathrm{P}$ & SPB & 1.031 & & & 6.449 & 0.057 & B1V \\
\hline DQ Cru & 110863 & 62291 & U & $\mathrm{L}$ & & & & 9.122 & 0.175 & B1Vp \\
\hline & 115034 & 64716 & & IA & & & & 8.821 & 0.091 & B1V \\
\hline & 154445 & 83635 & M & IA & & & & 5.671 & 0.033 & B1V \\
\hline BE Cap & 191639 & 99457 & $\mathrm{U}$ & IA & & & & 6.398 & 0.077 & B1V \\
\hline V417 Cep & 198895 & 102926 & $\mathrm{U}$ & $\mathrm{L}$ & & & & 8.416 & 0.173 & B1V \\
\hline V1931 Cyg & 200310 & 103732 & $\mathrm{U}$ & BCEP? & & 0.300 & 0.300 & 5.345 & 0.055 & B1V \\
\hline & & 107456 & U & $\mathrm{L}$ & & & & 9.094 & 0.135 & B1V: \\
\hline V439 Cep & 209145 & 108546 & $\mathrm{U}$ & $\mathrm{L}$ & & & & 7.734 & 0.107 & B1V \\
\hline V422 Lac & 216092 & 112698 & $\mathrm{U}$ & $\mathrm{L}$ & & & & 7.813 & 0.064 & B1V \\
\hline V454 Cep & 216711 & 113065 & $\mathrm{U}$ & IA? & & & & 9.187 & 0.154 & B1V \\
\hline V503 Car & 90578 & 51063 & $\mathrm{U}$ & $\mathrm{L}$ & & & & 9.342 & 0.116 & B1.5III \\
\hline V906 Cen & 306387 & 55499 & $\mathrm{U}$ & IA & & & 0.208 & 9.642 & 0.143 & B1.5III \\
\hline alf Lup & 129056 & 71860 & $\mathrm{P}$ & BCEP & 0.260 & & & 2.228 & 0.031 & B1.5III \\
\hline & 73 & 470 & $\mathrm{U}$ & IA & & & & 8.394 & 0.121 & B $1.5 \mathrm{IV}$ \\
\hline MZ Aur & 34626 & 24938 & $\mathrm{U}$ & IA & & & & 8.200 & 0.143 & B1.5IVnp \\
\hline V372 Car & 64722 & 38438 & $\mathrm{P}$ & BCEP & 0.115 & & & 5.642 & 0.030 & B1.5IV \\
\hline & 136298 & 75141 & M & IA & & & & 3.143 & 0.026 & B1.5IV \\
\hline
\end{tabular}


Table A.2. continued.

\begin{tabular}{|c|c|c|c|c|c|c|c|c|c|c|c|}
\hline $\begin{array}{l}\text { Var. } \\
\text { name }\end{array}$ & $\begin{array}{l}\mathrm{HD} \\
N_{\mathrm{o}} .\end{array}$ & $\begin{array}{l}\mathrm{HIP} \\
N_{\mathrm{o}} .\end{array}$ & $\begin{array}{l}\text { Var. } \\
\text { flag }\end{array}$ & $\begin{array}{l}\text { Var. } \\
\text { type }\end{array}$ & $\begin{array}{l}\text { HIP } \\
P(\mathrm{~d})\end{array}$ & $\begin{array}{l}\text { New } \\
P(\mathrm{~d})\end{array}$ & & $\begin{array}{c}\text { CK } \\
P(\mathrm{~d})\end{array}$ & $\begin{array}{c}\left\langle H_{\mathrm{p}}\right\rangle \\
(\mathrm{mag})\end{array}$ & $\begin{array}{l}\text { Ampl. } \\
\text { (mag) }\end{array}$ & $\begin{array}{l}\text { Sp. } \\
\text { type }\end{array}$ \\
\hline lam Sco & 158926 & 85927 & $\mathrm{P}$ & BCEP & 0.214 & & & & 1.525 & 0.054 & $\mathrm{~B} 1.5 \mathrm{IV}+\ldots$ \\
\hline V1378 Ori & 37334 & 26442 & $\mathrm{P}$ & SPB & 2.437 & & & & 7.103 & 0.063 & $\mathrm{~B} 1.5 \mathrm{~V}$ \\
\hline V916 Cen & 101794 & 57106 & $\mathrm{U}$ & $\mathrm{L}$ & & & & & 8.704 & 0.203 & $\mathrm{~B} 1.5 \mathrm{~V}$ \\
\hline V2123 Cyg & 195907 & 101411 & $\mathrm{U}$ & $\mathrm{L}$ & & & & & 7.799 & 0.133 & $\mathrm{~B} 1.5 \mathrm{~V}$ \\
\hline V787 Cas & 13590 & 10486 & $\mathrm{U}$ & L/IA? & & 13.433 & & & 8.065 & 0.238 & B2III \\
\hline \multirow[t]{2}{*}{ nu. Eri } & 29248 & 21444 & $\mathrm{P}$ & BCEP & 0.174 & & & & 3.871 & 0.119 & B2III SB \\
\hline & 66396 & 39331 & & IA & & & & & 8.929 & 0.088 & B2III \\
\hline V426 Pup & 68570 & 40148 & $\mathrm{U}$ & $\mathrm{L}$ & & & & & 7.754 & 0.132 & B2III \\
\hline NSV 04232 & 74824 & 42923 & $\mathrm{U}$ & IA & & & & 2.737 & 5.691 & 0.053 & B2III \\
\hline Bw Cru & & 62949 & & IA & & & & & 9.081 & 0.096 & B2III \\
\hline & 120928 & 67912 & $\mathrm{U}$ & $\mathrm{L}$ & & & & 12.092 & 9.818 & 0.136 & B2III: \\
\hline BU Cir & 129557 & 72121 & $\mathrm{P}$ & BCEP & 0.128 & & & & 6.083 & 0.034 & B2III \\
\hline & 159792 & 86363 & & IA & & & & & 9.497 & 0.116 & B2III \\
\hline mu. Sgr & 166937 & 89341 & $\mathrm{U}$ & SPB? & & 1.746 & & & 3.881 & 0.112 & B2III: \\
\hline BW Vul & 199140 & 103191 & $\mathrm{P}$ & BCEP & 0.201 & & & & 6.522 & 0.229 & B2IIIvar \\
\hline V421 Cер & 203025 & 105091 & $\mathrm{U}$ & IA & & & & & 6.477 & 0.062 & B2III \\
\hline DD Lac & 214993 & 112031 & $\mathrm{P}$ & BCEP & 0.193 & & & & 5.195 & 0.117 & B2IIIv SB \\
\hline KV CMa & 50118 & 32856 & $\mathrm{U}$ & IA? & & & & & 7.112 & 0.116 & B2III/IV \\
\hline & 67924 & 39894 & $\mathrm{U}$ & IA & & & & & 7.723 & 0.090 & B2III/IV \\
\hline del Cet & 16582 & 12387 & $\mathrm{P}$ & BCEP & 0.161 & & & & 4.006 & 0.031 & B2IV \\
\hline KP Per & 21803 & 16516 & $\mathrm{P}$ & BCEP & 0.202 & & & & 6.426 & 0.104 & B2IV \\
\hline lam Eri & 33328 & 23972 & U & SPB? & & 2.959 & & & 4.186 & 0.084 & B2IVn \\
\hline V901 Ori & 37776 & 26742 & $\mathrm{P}$ & SPB & 1.539 & & & & 6.950 & 0.044 & B2IV \\
\hline & 55856 & 34940 & $\mathrm{U}$ & IA? & & 0.088 & & 0.088 & 6.273 & 0.049 & B2IV \\
\hline TY Crv & 104337 & 58587 & $P$ & SPB & 1.481 & & & & 5.211 & 0.056 & B2IV \\
\hline tau01 Lup & 126341 & 70574 & $\mathrm{P}$ & BCEP & 0.177 & & & & 4.509 & 0.038 & B2IV \\
\hline gam Lup & 138690 & 76297 & $\mathrm{P}$ & SPB & 2.851 & & & & 2.701 & 0.026 & B2IV \\
\hline V848 Ara & 152979 & 83105 & U & $\mathrm{L}$ & & & & & 8.144 & 0.191 & B2IV \\
\hline the Oph & 157056 & 84970 & $\mathrm{P}$ & BCEP & 0.141 & & & & 3.194 & 0.030 & B2IV \\
\hline V4372 Sgr & 183133 & 95755 & $\mathrm{P}$ & SPB & 1.018 & & & & 6.768 & 0.042 & B2IV \\
\hline V2139 Cyg & 199356 & 103277 & $\mathrm{U}$ & $\mathrm{L}$ & & & & & 7.192 & 0.136 & B2IVp: \\
\hline EN Lac & 216916 & 113281 & $\mathrm{P}$ & BCEP & 0.171 & & & & 5.550 & 0.039 & B2IV \\
\hline & 6226 & 4983 & U & IA & & & & & 6.798 & 0.104 & B2IV-V \\
\hline V433 Aur & 37367 & 26606 & $\mathrm{P}$ & SPB? & 4.638 & 4.639 & 3.678 & & 6.032 & 0.043 & B2IV-V \\
\hline MM CMa & 55522 & 34798 & $\mathrm{P}$ & SPB? & 2.729 & & & & 5.859 & 0.050 & B2IV/V \\
\hline & 56995 & 35370 & & IA & & & & & 8.878 & 0.093 & B2IV/V \\
\hline & 68217 & 39961 & $\mathrm{U}$ & IA & & & & & 5.133 & 0.028 & B2IV-V \\
\hline V2052 Oph & 163472 & 87812 & $\mathrm{P}$ & BCEP & 0.140 & & & & 5.849 & 0.043 & B2IV-V \\
\hline IN Peg & 212076 & 110386 & $\mathrm{U}$ & $\mathrm{L}$ & & & & & 4.775 & 0.278 & B2IV-V \\
\hline & 232819 & 17200 & $\mathrm{U}$ & IA & & & & & 9.446 & 0.124 & $\mathrm{~B} 2 \mathrm{~V}$ \\
\hline & 39478 & 27881 & $\mathrm{U}$ & BCEP? & & 0.489 & & & 8.270 & 0.078 & B2V \\
\hline & 41534 & 28756 & M & ? & & 12.348 & 8.850 & 8.837 & 5.582 & 0.030 & B2V \\
\hline & 46547 & 31190 & U & SPB? & & 1.681 & & & 5.657 & 0.058 & B2V \\
\hline FT CMa & 48917 & 32292 & U & SPB? & & 1.168 & & & 5.182 & 0.106 & $\mathrm{~B} 2 \mathrm{~V}$ \\
\hline & 51285 & 33309 & $\mathrm{U}$ & IA? & & 1.732 & & & 8.086 & 0.075 & B2V(n) \\
\hline & 52812 & 33846 & U & IA & & & & & 6.891 & 0.046 & B2V \\
\hline FV CMa & 54309 & 34360 & U & $\mathrm{L}$ & & & & & 5.725 & 0.286 & B2V:nn \\
\hline NSV 04058 & 71130 & 41037 & $\mathrm{U}$ & $\mathrm{L}$ & & & & & 1.999 & 0.071 & $\mathrm{~B} 2 \mathrm{~V}$ \\
\hline V335 Vel & 85953 & 48527 & $\mathrm{P}$ & SPB & 3.756 & & & & 5.893 & 0.040 & $\mathrm{~B} 2 \mathrm{~V}$ \\
\hline & 93684 & 52766 & U & IA & & & & & 7.687 & 0.068 & B2V \\
\hline & 103574 & 58128 & $\mathrm{U}$ & IA & & & & & 7.983 & 0.076 & B2V \\
\hline & 124298 & 69617 & $\mathrm{U}$ & IA & & & & & 9.621 & 0.132 & $\mathrm{~B} 2 \mathrm{~V}$ \\
\hline V761 Cen & 125823 & 70300 & $\mathrm{P}$ & SPB? & 8.812 & & & & 4.350 & 0.067 & $\mathrm{~B} 2 \mathrm{~V}$ \\
\hline & 170740 & 90804 & $\mathrm{U}$ & IA & & & & & 5.806 & 0.036 & B2V \\
\hline & 172910 & 91918 & $\mathrm{U}$ & SPB? & & 1.199 & & 1.198 & 4.795 & 0.026 & B2V \\
\hline V4024 Sgr & 178175 & 93996 & U & IA & & & & & 5.538 & 0.046 & B2V \\
\hline & 182180 & 95408 & $\mathrm{U}$ & IA & & & & 0.261 & 5.995 & 0.048 & B2Vnn \\
\hline V396 Vul & 187851 & 97681 & $\mathrm{P}$ & BCEP & 0.410 & & & & 7.797 & 0.079 & B2V:nn \\
\hline & 209454 & 108720 & $\mathrm{U}$ & $?$ & & 25 & & 254.453 & 7.839 & 0.062 & $\mathrm{~B} 2 \mathrm{~V}$ \\
\hline V365 Lac & 209961 & 109082 & $\mathrm{P}$ & SPB & 1.086 & & & & 6.238 & 0.075 & B2V SB \\
\hline & 214652 & 111828 & M & $\mathrm{L}$ & & & & & 6.791 & 0.040 & B2:V SB \\
\hline V399 Cep & & 386 & $\mathrm{U}$ & L/IA? & & & & & 9.245 & 0.212 & B2 \\
\hline V771 Cas & & 7936 & $\mathrm{U}$ & IA & & & & & 9.382 & 0.164 & B2 \\
\hline & & 17146 & $\mathrm{U}$ & IA & & & & & 9.288 & 0.107 & B2 \\
\hline V836 Cen & 129929 & 72241 & $\mathrm{U}$ & IA & & & & & 8.030 & 0.069 & B2 \\
\hline V1018 Cen & 130903 & 72710 & $\mathrm{P}$ & SPB & 1.313 & & & & 7.927 & 0.121 & B2:p \\
\hline V2172 Cyg & 235668 & 108326 & $\mathrm{U}$ & $\mathrm{L}$ & & & & & 8.225 & 0.127 & B2 \\
\hline LS CMa & 52670 & 33804 & U & IA & & & & & 5.584 & 0.061 & B2/B3III/IV \\
\hline V408 Pup & 65719 & 39020 & U & IA & & & & & 9.288 & 0.153 & B2/B3III \\
\hline & 101795 & 57108 & $\mathrm{U}$ & IA & & & & 5.507 & 7.330 & 0.065 & B2/B3III \\
\hline & 121531 & 68222 & D & IA? & & & & & 9.903 & 0.124 & B2/B3III \\
\hline V692 CrA & 166596 & 89290 & $\mathrm{U}$ & IA & & & & 0.689 & 5.415 & 0.071 & B2.5III \\
\hline & 40494 & 28199 & $\mathrm{U}$ & $\mathrm{L}$ & & & & & 4.305 & 0.044 & B2.5IV \\
\hline EO Leo & 87015 & 49220 & $\mathrm{P}$ & SPB? & 2.779 & & & & 5.609 & 0.037 & B2.5IV \\
\hline V1003 Sco & 149711 & 81472 & $\mathrm{U}$ & IA & & & & & 5.817 & 0.039 & B2.5IV \\
\hline V2014 Cyg & 195556 & 101138 & $\mathrm{U}$ & $\mathrm{L}$ & & & & 1.137 & 4.918 & 0.034 & B2.5IV \\
\hline NSV 02891 & 43544 & 29771 & $\mathrm{U}$ & IA & & & & & 5.908 & 0.054 & B2/B3V \\
\hline & 46994 & 31436 & $\mathrm{U}$ & IA & & & & & 7.806 & 0.061 & B2/B3V \\
\hline & 52597 & 33769 & U & IA & & & & & 7.785 & 0.067 & B2/B3V \\
\hline V378 Pup & 60855 & 36981 & $\mathrm{U}$ & SPB? & & 1.020 & & & 5.632 & 0.086 & $\mathrm{~B} 2 / 3 \mathrm{~V}(\mathrm{n})$ \\
\hline V403 Pup & 64298 & 38439 & U & IA & & & & & 9.068 & 0.106 & B2/B3V:nn \\
\hline V375 Car & 67536 & 39530 & $\mathrm{P}$ & SPB & 1.016 & & & & 6.223 & 0.103 & $\mathrm{~B} 2.5 \mathrm{Vn}$ \\
\hline V1008 Cen & 127449 & 71194 & $\mathrm{U}$ & IA & & & & & 7.741 & 0.097 & B2/B3Vn \\
\hline CO Cir & 129954 & 72438 & $\mathrm{U}$ & $\mathrm{L}$ & & & & & 5.867 & 0.124 & $\mathrm{~B} 2.5 \mathrm{~V}$ \\
\hline
\end{tabular}


Table A.2. continued.

\begin{tabular}{|c|c|c|c|c|c|c|c|c|c|c|c|}
\hline $\begin{array}{r}\text { Var. } \\
\text { name }\end{array}$ & $\begin{array}{l}\mathrm{HD} \\
N_{\mathrm{o}} .\end{array}$ & $\begin{array}{l}\mathrm{HIP} \\
N_{\mathrm{o}} .\end{array}$ & $\begin{array}{l}\text { Var. } \\
\text { flag }\end{array}$ & $\begin{array}{l}\text { Var. } \\
\text { type }\end{array}$ & $\begin{array}{l}\text { HIP } \\
P(\mathrm{~d})\end{array}$ & $\begin{array}{l}\text { New } \\
P(\mathrm{~d})\end{array}$ & & $\begin{array}{l}\text { CK } \\
P(\mathrm{~d})\end{array}$ & $\begin{array}{c}\left.H_{\mathrm{p}}\right\rangle \\
(\mathrm{mag})\end{array}$ & $\begin{array}{c}\text { Ampl. } \\
\text { (mag) }\end{array}$ & $\begin{array}{l}\text { Sp. } \\
\text { type }\end{array}$ \\
\hline alf Sco & 148478 & 80763 & $\mathrm{U}$ & $\mathrm{L}$ & & & & & 0.985 & 0.151 & B2.5V \\
\hline CX Dra & 174237 & 92133 & $\mathrm{U}$ & $\mathrm{L}$ & & & & 1.273 & 5.866 & 0.178 & B2.5V \\
\hline V341 Sge & 186272 & 96984 & $\mathrm{U}$ & $\mathrm{L}$ & & & & & 7.803 & 0.144 & B $2.5 \mathrm{~V}$ \\
\hline V395 Vul & 187811 & 97679 & $\mathrm{U}$ & $\mathrm{L}$ & & & & & 4.846 & 0.123 & B $2.5 \mathrm{~V}$ \\
\hline \multirow[t]{2}{*}{ V1624 Cyg } & 191610 & 99303 & $\mathrm{U}$ & $\mathrm{L}$ & & & & & 4.881 & 0.092 & B2.5V \\
\hline & 124327 & 69633 & $\mathrm{U}$ & IA & & & & 55.991 & 8.593 & 0.104 & B2/B3 \\
\hline V760 Cas & 6417 & 5161 & $\mathrm{P}$ & SPB? & 1.932 & & & & 7.135 & 0.077 & B3III \\
\hline V737 Mon & 48282 & 32088 & $\mathrm{U}$ & L? & & & & & 8.862 & 0.112 & B3III \\
\hline EW CMa & 56014 & 34981 & $\mathrm{U}$ & $\mathrm{L}$ & & & & & 4.382 & 0.058 & B3III \\
\hline & 61207 & 37113 & $\mathrm{U}$ & IA & & & & & 8.086 & 0.070 & B3III \\
\hline & 72771 & 41980 & $\mathrm{U}$ & IA & & & & & 7.829 & 0.065 & B3III \\
\hline V485 Car & 84375 & 47549 & $\mathrm{U}$ & IA & & & & & 7.093 & 0.052 & B3III:psh \\
\hline LX Vel & 91188 & 51444 & $\mathrm{U}$ & $\mathrm{L}$ & & & & 1030.928 & 6.590 & 0.124 & B3III \\
\hline & 116053 & 65223 & $\mathrm{U}$ & IA & & & & & 7.750 & 0.074 & B3III \\
\hline & 157455 & 85308 & $\mathrm{U}$ & SPB? & & 2.750 & & 2.748 & 6.881 & 0.054 & B3III \\
\hline & 167233 & 89502 & $\mathrm{U}$ & IA & & & & & 6.899 & 0.083 & B3III \\
\hline & 67059 & 39508 & $\mathrm{U}$ & $\mathrm{L}$ ? & & 10.529 & & & 7.713 & 0.126 & B3III/IV \\
\hline V420 Pup & 67698 & 39834 & $\mathrm{U}$ & $\mathrm{L}$ & & & & & 6.481 & 0.303 & B3III/IV \\
\hline & 124834 & 70042 & M & IA & & & & & 6.613 & 0.038 & B3III/IV \\
\hline omi Vel & 74195 & 42536 & $\mathrm{P}$ & SPB/IA & 2.798 & & & & 3.535 & 0.050 & B3IV \\
\hline IY Vel & 76566 & 43807 & $\mathrm{U}$ & SPB? & & 0.921 & & & 6.210 & 0.049 & B3IV \\
\hline V514 Car & 92287 & 52043 & $\mathrm{P}$ & SPB & 1.453 & & & & 5.848 & 0.039 & B3IV \\
\hline V817 Cen & 105521 & 59232 & $\mathrm{U}$ & L? & & 42.840 & & 42.409 & 5.468 & 0.185 & B3IV \\
\hline & 116862 & 65630 & $\mathrm{U}$ & SPB? & & 1.582 & & & 6.231 & 0.047 & B3IV \\
\hline & 129123 & 71953 & $\mathrm{U}$ & IA & & & & & 8.879 & 0.135 & B3IV \\
\hline MQ TrA & 143448 & 78682 & $\mathrm{U}$ & L & & & & & 7.104 & 0.387 & B3IV \\
\hline & 164852 & 88331 & M & IA? & & 1.381 & & 1.261 & 5.225 & 0.029 & B3IV \\
\hline & 189687 & 98425 & M & SPB? & & 2.331 & 5.128 & & 5.106 & 0.028 & B3IV \\
\hline & 239683 & 106134 & $\mathrm{U}$ & IA & & & & & 9.407 & 0.107 & B3IV \\
\hline KY And & 218674 & 114329 & $\mathrm{U}$ & IA & & & & & 6.748 & 0.056 & B3IV SB: \\
\hline & 13669 & 10475 & $\mathrm{U}$ & IA & & & & & 8.403 & 0.090 & B3IV-V \\
\hline V994 Sco & 160124 & 86432 & $\mathrm{P}$ & SPB & 1.917 & & & & 7.178 & 0.108 & $\mathrm{~B} 3 \mathrm{IV} / \mathrm{V}$ \\
\hline Alf Eri & 10144 & 7588 & $\mathrm{U}$ & IA? & & 3.731 & & 3.731 & 0.426 & 0.064 & B3Vp \\
\hline NSV 01089 & 20340 & 15188 & $\mathrm{U}$ & IA & & & & & 7.938 & 0.092 & B3V \\
\hline & 21428 & 16244 & D & IA? & & & & & 4.643 & 0.087 & B3V \\
\hline & 23466 & 17563 & $\mathrm{U}$ & IA & & & & & 5.313 & 0.032 & B3V \\
\hline V1133 Tau & 25558 & 18957 & $\mathrm{P}$ & ELL/SP & 1.532 & & & & 5.299 & 0.046 & B3V \\
\hline & 36013 & 25648 & $\mathrm{U}$ & BCEP? & & 40.003 & 0.347 & & 6.851 & 0.055 & B3V:n \\
\hline V593 Tau & 39340 & 27808 & $\mathrm{U}$ & IA & & & & & 8.131 & 0.094 & B3V \\
\hline NSV 02931 & 44506 & 30143 & $\mathrm{U}$ & IA & & & & & 5.477 & 0.058 & B3V \\
\hline & 49260 & 32354 & U & IA & & & & & 7.171 & 0.061 & B3V \\
\hline $\mathrm{HZ} \mathrm{CMa}$ & 50123 & 32810 & $\mathrm{P}$ & $?$ & 14.302 & & & & 5.755 & 0.074 & B3V \\
\hline LQ CMa & 52356 & 33673 & U & IA & & & & & 7.171 & 0.125 & B3V(n) \\
\hline FU CMa & 52437 & 33721 & $\mathrm{P}$ & SPB & 1.109 & & & & 6.471 & 0.041 & B3Vnn \\
\hline IL CMa & 54031 & 34248 & $\mathrm{U}$ & IA? & & 0.178 & & 0.089 & 6.295 & 0.065 & B3V \\
\hline & 53857 & 34262 & U & IA & & & & & 8.274 & 0.183 & B3V \\
\hline & 57593 & 35611 & $\mathrm{U}$ & IA & & & & & 5.938 & 0.052 & B3V V \\
\hline NO CMa & 58155 & 35795 & $\mathrm{U}$ & IA & & & & & 5.359 & 0.045 & B3V \\
\hline NSV 03629 & 60098 & 36582 & $\mathrm{U}$ & IA? & & 7.195 & & & 6.626 & 0.053 & B3V \\
\hline & 62663 & 37533 & U & $\mathrm{L}$ & & & & 934.579 & 6.912 & 0.068 & B3V \\
\hline MX Vel & 67341 & 39584 & U & $\mathrm{I} / \mathrm{L}$ ? & & & & & 6.133 & 0.070 & B3Vnp \\
\hline PQ Pup & 67888 & 39866 & $\mathrm{U}$ & $\mathrm{L}$ & & & & & 6.334 & 0.150 & B3V \\
\hline AI Pyx & 75112 & 43114 & U & IA & & & & & 6.303 & 0.140 & B3V \\
\hline V492 Car & 86659 & 48782 & $\mathrm{P}$ & SPB? & 1.062 & & & & 6.157 & 0.046 & B3V \\
\hline V518 Car & 92938 & 52370 & $\mathrm{U}$ & $\mathrm{L}$ & & 100 & & 970.874 & 4.693 & 0.198 & B3V \\
\hline & 99872 & 55979 & U & SPB? & & 1.504 & & & 6.133 & 0.033 & B3V \\
\hline NSV 05357 & 102776 & 57669 & $\mathrm{U}$ & $\mathrm{L}$ & & & & & 4.270 & 0.049 & B3V \\
\hline KV Mus & 106881 & 59959 & $\mathrm{U}$ & $\mathrm{L}$ & & & & & 8.559 & 0.142 & B3Vnn \\
\hline KZ Mus & 109885 & 61751 & $\mathrm{P}$ & BCEP & 0.171 & & & & 9.058 & 0.105 & B3V \\
\hline & 129092 & 72000 & U & L/IA? & & & & & 6.358 & 0.066 & B3V: \\
\hline & 132955 & 73624 & & IA & & & & & 5.409 & 0.030 & B3V \\
\hline CU Cir & 133495 & 74011 & $\mathrm{U}$ & SPB? & & 2.066 & & & 8.550 & 0.092 & B3 $3 n \ldots$ \\
\hline & 133699 & 74110 & U & IA & & & & & 6.800 & 0.058 & B3V \\
\hline & 135737 & 75091 & $\mathrm{D}$ & SPB? & & 1.497 & & & 6.249 & 0.034 & B3V \\
\hline & 142883 & 78168 & U & IA & & & & 0.095 & 5.857 & 0.084 & B3V \\
\hline iot Her & 160762 & 86414 & $\mathrm{P}$ & SPB & 3.487 & & & & 3.750 & 0.026 & B3V SB \\
\hline V543 Lyr & 176502 & 93177 & $\mathrm{U}$ & SPB? & & 23.809 & 1.859 & 0.093 & 6.145 & 0.103 & $\mathrm{~B} 3 \mathrm{~V}$ \\
\hline V550 Lyr & 178329 & 93808 & $\mathrm{P}$ & SPB & 1.687 & & & & 6.452 & 0.059 & B3V \\
\hline NSV 11808 & 179406 & 94385 & U & IA & & & & & 5.385 & 0.030 & B3V \\
\hline QR Vul & 192685 & 99824 & $\mathrm{U}$ & SPB? & & 6.321 & & & 4.707 & 0.075 & B3V \\
\hline V381 Cep & 203338 & 105259 & $\mathrm{U}$ & IA & & & & 51.020 & 5.651 & 0.078 & B $3 V_{v}$ comp \\
\hline V433 Cep & 206135 & 106712 & $\mathrm{U}$ & $\mathrm{L}$ & & & & & 8.307 & 0.156 & B3V \\
\hline eps Cap & 205637 & 106723 & $\mathrm{U}$ & SPB? & & 1.570 & & & 4.465 & 0.165 & B3V:p \\
\hline OQ Peg & 208057 & 108022 & $\mathrm{U}$ & SPB? & & 1.247 & 1.048 & 1.247 & 5.038 & 0.038 & B3V \\
\hline UU PsA & 209522 & 108975 & $\mathrm{U}$ & IA & & & & & 5.912 & 0.032 & B3V (+B) \\
\hline V423 Lac & 216851 & 113226 & U & $\mathrm{L}$ & & & & & 8.004 & 0.084 & B3V:n \\
\hline V378 And & 217543 & 113640 & $\mathrm{U}$ & $\mathrm{L}$ & & & & & 6.533 & 0.101 & B3Vp \\
\hline & 223145 & 117315 & & $\mathrm{~L}$ & & & & 2.966 & 5.119 & 0.026 & B3V \\
\hline & 232161 & 1621 & D & IA? & & & & & 8.948 & 0.097 & B3 \\
\hline & 236737 & 6775 & $\mathrm{U}$ & IA & & & & & 8.585 & 0.085 & B3 \\
\hline & 236875 & 8313 & U & IA & & & & 56.689 & 9.500 & 0.172 & B3 \\
\hline V788 Cas & 15472 & 11894 & $\mathrm{P}$ & ELL/SP & 3.038 & & & & 7.901 & 0.074 & B3 \\
\hline V1156 Ori & 35298 & 25235 & $\mathrm{P}$ & SXARI & 1.854 & & & & 7.874 & 0.062 & B3vw He wk \\
\hline & 46339 & 31196 & $\mathrm{U}$ & IA & & & & & 9.008 & 0.104 & B3 \\
\hline
\end{tabular}


Table A.2. continued.

\begin{tabular}{|c|c|c|c|c|c|c|c|c|c|c|}
\hline $\begin{array}{r}\text { Var. } \\
\text { name }\end{array}$ & $\begin{array}{l}\mathrm{HD} \\
N_{\mathrm{o}} .\end{array}$ & $\begin{array}{l}\mathrm{HIP} \\
N_{\mathrm{o}} .\end{array}$ & $\begin{array}{l}\text { Var. } \\
\text { flag }\end{array}$ & $\begin{array}{l}\text { Var. } \\
\text { type }\end{array}$ & $\begin{array}{l}\text { HIP } \\
P(\mathrm{~d})\end{array}$ & $\begin{array}{l}\text { New } \\
P(\mathrm{~d})\end{array}$ & $\begin{array}{l}\text { CK } \\
P(\mathrm{~d})\end{array}$ & $\begin{array}{c}\left\langle H_{\mathrm{p}}\right\rangle \\
(\mathrm{mag})\end{array}$ & $\begin{array}{c}\text { Ampl. } \\
\text { (mag) }\end{array}$ & $\begin{array}{l}\text { Sp. } \\
\text { type }\end{array}$ \\
\hline V1448 Aql & 180126 & 94596 & $\mathrm{U}$ & $\mathrm{L}$ & & & & 8.036 & 0.191 & B3p \\
\hline V2149 Cyg & & 105010 & $\mathrm{U}$ & $\mathrm{L}$ & & & & 8.872 & 0.210 & B3:nnpsh \\
\hline V379 And & 218325 & 114100 & $\mathrm{U}$ & $\mathrm{L}$ & & & & 7.768 & 0.132 & B3 \\
\hline V380 Pup & 61328 & 37099 & $\mathrm{U}$ & IA & & & 0.811 & 9.489 & 0.430 & $\mathrm{~B} 3 / 4 \mathrm{~V}+\mathrm{B} 8 / 9$ \\
\hline & 85860 & 48547 & D & IA & & & & 7.018 & 0.056 & $\mathrm{~B} 3 / 5 \mathrm{~V}+\mathrm{B} / \mathrm{A}$ \\
\hline
\end{tabular}

This table is the appendixed version of Table 5.

The columns are: the variable name, the HD number, the HIPPARCOS number, the HIPPARCOS variability flag, the variability type we assigned them in this study, the HIPPARCOS period (if any), the new periods found in this study ( 2 columns, for a possible second period), the periods found by Koen \& Eyer (2002) (if any), the $H p$ magnitude, the mean amplitude calculated here and the spectral type according to the Vizier database.

The classification is based on the variable types from the GCVS (Samus et al. 2004). The question mark means that the classification is either based on a new period (thus unsure) or that the type cannot be clearly identified from the HIPPARCOS lightcurve.

${ }^{a}$ HIP34301 is reported to have a period $P=0.123 \mathrm{~d}$.

${ }^{b}$ HIP94827 is reported to have a period $P=0.609 \mathrm{~d}$.

${ }^{c}$ HIP68702 is reported to have a period $P=0.157 \mathrm{~d}$.

${ }^{d}$ HIP10055 is reported to have a period $P=0.374$ d. 
Table A.3. OBe stars above the threshold $T_{\mathrm{h}}$.

\begin{tabular}{|c|c|c|c|c|c|c|c|c|c|c|c|}
\hline $\begin{array}{l}\text { Var. } \\
\text { name }\end{array}$ & $\begin{array}{l}\mathrm{HD} \\
N_{\mathrm{o}} .\end{array}$ & $\begin{array}{l}\mathrm{HIP} \\
N_{\mathrm{o}} .\end{array}$ & $\begin{array}{l}\text { Var. } \\
\text { flag }\end{array}$ & $\begin{array}{l}\text { Var. } \\
\text { type }\end{array}$ & $\begin{array}{l}\text { HIP } \\
P(\text { d) }\end{array}$ & $\begin{array}{l}\text { New } \\
P(\mathrm{~d})\end{array}$ & & $\begin{array}{l}\text { CK } \\
P(\mathrm{~d})\end{array}$ & $\begin{array}{c}\left\langle H_{\mathrm{p}}\right\rangle \\
(\mathrm{mag})\end{array}$ & $\begin{array}{l}\text { Ampl. } \\
\text { (mag) }\end{array}$ & $\begin{array}{l}\text { Sp. } \\
\text { type }\end{array}$ \\
\hline \multirow[t]{2}{*}{ V1382 Ori } & 39680 & 27941 & $\mathrm{U}$ & $\mathrm{L}$ & & & & & 7.901 & 0.131 & O6:pe SB \\
\hline & & 101425 & D & IA & & & & & 8.851 & 0.088 & O6e \\
\hline NSV 14069 & 210839 & 109556 & U & IA & & & & & 5.128 & 0.036 & O6e \\
\hline BN Gem & 60848 & 37074 & $\mathrm{U}$ & GCAS? & & 2.983 & & & 6.868 & 0.054 & O8V:pevar \\
\hline QZ Sge & 188001 & 97796 & $\mathrm{U}$ & IA & & & & & 6.232 & 0.047 & O8e \\
\hline & 192639 & 99768 & M & IA & & & & & 7.197 & 0.048 & O8e \\
\hline PZ Gem & 45314 & 30722 & $\mathrm{U}$ & IA & & & & & 6.617 & 0.043 & O9:pe \\
\hline & 24534 & 18350 & & IA & & & & & 6.825 & 0.044 & 09.5pe \\
\hline V783 Cas & & 10147 & $\mathrm{U}$ & IA? & & 6.330 & & 36.900 & 9.910 & 0.182 & Bpe \\
\hline V725 Tau & 245770 & 26566 & $\mathrm{U}$ & XNG/L & & & & & 9.267 & 0.256 & Bpe \\
\hline FS CMa & 45677 & 30800 & $\mathrm{U}$ & GCAS & & & & & 8.049 & 0.410 & Bpe (shell) \\
\hline QQ Gem & 46264 & 31236 & $\mathrm{U}$ & IA & & & & & 7.657 & 0.092 & $\mathrm{Be}$ \\
\hline $\mathrm{ZCMa}$ & 53179 & 34042 & $\mathrm{R}$ & IN/L & & & & & 9.765 & 0.346 & Bpe \\
\hline GG Car & 94878 & 53444 & $\mathrm{U}$ & $\mathrm{L} / \mathrm{IA} ?^{a}$ & & & & & 8.783 & 0.438 & Bep \\
\hline CQ Cir & 130437 & 72616 & $\mathrm{U}$ & $\mathrm{IA}^{b}$ & & & & & 9.976 & 0.132 & $\mathrm{Be}$ \\
\hline V4375 Sgr & 316285 & 87136 & U & IA & & & & & 9.016 & 0.119 & $\mathrm{Be}$ \\
\hline V1427 Cyg & & 106628 & $\mathrm{U}$ & $\mathrm{L}$ & & & & & 9.197 & 0.143 & Bpe \\
\hline KX And & 218393 & 114154 & U & GCAS? & & 38.856 & & 38.956 & 7.091 & 0.189 & Bpe \\
\hline gam Cas & 5394 & 4427 & U & GCAS & & & & & 2.138 & 0.029 & B0IV:evar \\
\hline V420 Aur & 34921 & 25114 & U & $\mathrm{I} / \mathrm{L}$ ? & & & & & 7.472 & 0.121 & B0IVpe \\
\hline V750 Mon & 53367 & 34116 & $\mathrm{U}$ & GCAS & & & & & 7.100 & 0.265 & B0IV:e \\
\hline & 166524 & 89158 & D & IA & & & & & 9.825 & 0.296 & B0Vpe \\
\hline & 206773 & 107164 & $\mathrm{U}$ & L? & & & & & 6.948 & 0.052 & B0V:pe \\
\hline V739 Mon & 49330 & 32586 & $\mathrm{U}$ & IA? & & 19.235 & 0.267 & 19.113 & 8.986 & 0.091 & B0:nnpe \\
\hline & 237056 & 14166 & $\mathrm{U}$ & IA & & & & & 8.971 & 0.093 & B0.5:V:pe \\
\hline & 122669 & 68817 & $\mathrm{U}$ & SPB? & & 0.951 & & 0.951 & 9.010 & 0.106 & B $0.5 \mathrm{Ve}$ \\
\hline CW Cir & 134958 & 74654 & $\mathrm{U}$ & IA & & & & & 8.233 & 0.106 & B0.5Vne \\
\hline V811 Cas & 220116 & 115244 & $\mathrm{P}$ & SPB? & 3.387 & & & & 8.753 & 0.223 & B $0.5 \mathrm{Vpe}$ \\
\hline V555 Per & 15450 & 11722 & $\mathrm{U}$ & IA & & & & & 9.062 & 0.183 & B1IIIe \\
\hline omi Pup & 63462 & 38070 & U & IA & & & & & 4.443 & 0.034 & B1IV:nne \\
\hline V415 Lac & 240010 & 111785 & U & $\mathrm{L}$ & & & & & 9.270 & 0.121 & B1:IV:nnpe \\
\hline V548 Per & & 9017 & $\mathrm{U}$ & L/IA? & & & & & 9.835 & 0.324 & B1V:pe \\
\hline V780 Cas & 12302 & 9538 & $\mathrm{U}$ & IA? & & 25.638 & & & 8.157 & 0.158 & B1:V:pe \\
\hline V801 Cas & 19243 & 14626 & $\mathrm{U}$ & L/IA? & & 25 & & 271.739 & 6.537 & 0.098 & B1V:e \\
\hline DE Cam & 25348 & 19008 & U & IA & & & & & 8.229 & 0.145 & B1Vnnpe \\
\hline V1153 Tau & 32190 & 23436 & U & $\mathrm{L} / \mathrm{IA}$ & & & & & 8.403 & 0.356 & B1Ve \\
\hline V413 Aur & 33152 & 24029 & U & $\mathrm{L}$ & & & & & 8.182 & 0.375 & B1Ve \\
\hline V1086 Ori & 35439 & 25302 & $\mathrm{U}$ & GCAS & & & & & 4.806 & 0.145 & B1V:pe \\
\hline V1163 Tau & 37318 & 26574 & $\mathrm{U}$ & IA & & & & & 8.474 & 0.118 & B1Vne \\
\hline V1165 Tau & 38010 & 26998 & $\mathrm{U}$ & IA & & & & & 6.815 & 0.080 & B1Vpe \\
\hline V1167 Tau & 248753 & 27850 & $\mathrm{U}$ & $\mathrm{I} / \mathrm{L}$ ? & & & & & 8.440 & 0.203 & B1Vnne \\
\hline FR CMa & 44458 & 30214 & $\mathrm{U}$ & GCAS & & & & & 5.539 & 0.116 & B1Vpe SB \\
\hline PY Gem & 44674 & 30452 & $\mathrm{U}$ & L? & & & & & 8.459 & 0.116 & B1Vne \\
\hline V801 Cen & 102567 & 57569 & $\mathrm{U}$ & GCAS & & & & & 8.936 & 0.256 & B1Vne \\
\hline LU Mus & 116849 & 65693 & $\mathrm{U}$ & IA & & & & & 9.227 & 0.108 & B1Vpe \\
\hline LV Mus & 117111 & 65848 & $\mathrm{U}$ & GCAS? & & & & 5.115 & 7.711 & 0.121 & B1Vpe \\
\hline V1059 Sco & 149313 & 81256 & U & GCAS & & & & & 9.528 & 0.319 & $\mathrm{~B} 1: \mathrm{Ve}$ \\
\hline V448 Sct & 170714 & 90768 & $\mathrm{U}$ & IA & & & & & 7.452 & 0.063 & B1Vne \\
\hline & 173219 & 91946 & $\mathrm{U}$ & IA & & & & & 7.891 & 0.063 & B1:V:npe \\
\hline V457 Sct & 174513 & 92510 & $\mathrm{U}$ & GCAS & & & & & 8.656 & 0.202 & B1V:npe \\
\hline V2113 Cyg & 193009 & 99953 & $\mathrm{U}$ & GCAS & & & & & 7.191 & 0.168 & B1V:nnpe \\
\hline V2135 Cyg & 198512 & 102700 & $\mathrm{U}$ & $\mathrm{L}$ & & & & & 8.227 & 0.115 & B1Vnnpe \\
\hline V2153 Cyg & 203731 & 105565 & $\mathrm{U}$ & IA & & & & & 7.565 & 0.095 & B1Vne \\
\hline V2155 Cyg & 204116 & 105699 & $\mathrm{U}$ & GCAS & & & & & 7.637 & 0.254 & $\mathrm{~B} 1 \mathrm{Ve}$ \\
\hline V357 Lac & 212044 & 110287 & $\mathrm{U}$ & GCAS & & & & & 7.263 & 0.122 & B1:V:nnpeva \\
\hline pi. Aqr & 212571 & 110672 & $\mathrm{U}$ & GCAS & & & & & 4.742 & 0.040 & B1Ve \\
\hline kap01 Aps & 137387 & 76013 & $\mathrm{U}$ & IA & & & & & 5.356 & 0.105 & B1npe \\
\hline V832 Cyg & 200120 & 103632 & $\mathrm{U}$ & BCEP? & & & & & 4.719 & 0.039 & Blne \\
\hline V810 Cas & 220058 & 115224 & $\mathrm{U}$ & $\mathrm{L}$ & & & & & 8.620 & 0.124 & B1npe \\
\hline LS Mus & 113120 & 63688 & $\mathrm{U}$ & GCAS & & & & & 5.944 & 0.103 & B1.5IIIne \\
\hline kap CMa & 50013 & 32759 & U & GCAS & & & & & 3.469 & 0.146 & B1.5IVne \\
\hline CV Cir & 133738 & 74147 & $\mathrm{U}$ & GCAS? & & 15.643 & & & 6.977 & 0.128 & B1/2IVne \\
\hline V581 Per & 24560 & 18424 & U & IA & & & & & 7.817 & 0.110 & B1.5Vne \\
\hline V907 Cen & 98927 & 55524 & $\mathrm{U}$ & ? & & 17.858 & & & 9.295 & 0.147 & $\mathrm{~B} 1.5 \mathrm{Ve}$ \\
\hline V397 Lac & & 109113 & $\mathrm{U}$ & IA & & & & & 9.732 & 0.118 & B1.5V:nne: \\
\hline LP CMa & 52244 & 33676 & $\mathrm{U}$ & IA & & & & & 9.246 & 0.107 & $\mathrm{~B} 1 / \mathrm{B} 2 \mathrm{e}$ \\
\hline V764 Cas & 7636 & 6027 & $\mathrm{U}$ & IA? & & 6.294 & & 6.294 & 6.886 & 0.078 & B2IIIne $+\ldots$ \\
\hline AX Mon & 45910 & 31019 & $\mathrm{U}$ & $\mathrm{I} / \mathrm{IA}$ & & & & & 6.757 & 0.152 & B2:IIIpshev \\
\hline V337 Vel & 88263 & 49743 & $\mathrm{U}$ & GCAS & & & & & 7.972 & 0.304 & B2IIIe \\
\hline V863 Cen & 105382 & 59173 & $\mathrm{P}$ & SPB: & 1.295 & & & & 4.405 & 0.040 & B2IIIne \\
\hline V767 Cen & 120991 & 67861 & $\mathrm{U}$ & GCAS? & & & & 4.260 & 5.851 & 0.270 & B2IIIe \\
\hline iot Ara & 157042 & 85079 & $\mathrm{U}$ & GCAS & & & & & 5.208 & 0.054 & B2IIIne \\
\hline V374 Car & 66194 & 38994 & U & GCAS & & & & & 5.760 & 0.110 & B2IVnpe \\
\hline V345 Car & 78764 & 44626 & $\mathrm{P}$ & $?$ & 137.700 & & & & 4.610 & 0.101 & B2IVe \\
\hline QY Car & 88661 & 49934 & $\mathrm{U}$ & GCAS & & & & & 5.716 & 0.123 & B2IVnpe \\
\hline del Cen & 105435 & 59196 & $\mathrm{U}$ & GCAS & & & & & 2.511 & 0.138 & B2IVne \\
\hline V952 Cen & 114441 & 64359 & $\mathrm{U}$ & IA & & & & 0.841 & 8.064 & 0.081 & B2IVpe \\
\hline V828 Ara & 153261 & 83323 & $\mathrm{U}$ & SPB? & & 6.098 & & 6.087 & 6.224 & 0.090 & B2IVne \\
\hline V549 Per & 13661 & 10463 & $\mathrm{U}$ & IA & & & & & 7.850 & 0.072 & B2IV-Ve \\
\hline V960 Tau & 36576 & 26064 & $\mathrm{P}$ & SPB & 1.037 & & & & 5.655 & 0.095 & B2IV-Ve \\
\hline ome $\mathrm{CMa}$ & 56139 & 35037 & $\mathrm{U}$ & GCAS & & & & & 3.968 & 0.213 & $\mathrm{~B} 2 \mathrm{IV} / \mathrm{Ve}$ \\
\hline mu. Cen & 120324 & 67472 & $\mathrm{U}$ & GCAS & & & & & 3.390 & 0.064 & B2IV-Ve \\
\hline V568 Cyg & 197419 & 102195 & $\mathrm{U}$ & GCAS & & & & & 6.633 & 0.082 & B2IV-Ve \\
\hline
\end{tabular}


Table A.3. continued.

\begin{tabular}{|c|c|c|c|c|c|c|c|c|c|c|}
\hline $\begin{array}{r}\text { Var. } \\
\text { name }\end{array}$ & $\begin{array}{l}\mathrm{HD} \\
N_{\mathrm{o}} .\end{array}$ & $\begin{array}{l}\mathrm{HIP} \\
N_{\mathrm{o}} .\end{array}$ & $\begin{array}{l}\text { Var. } \\
\text { flag }\end{array}$ & $\begin{array}{l}\text { Var. } \\
\text { type }\end{array}$ & $\begin{array}{l}\text { HIP } \\
P(\mathrm{~d})\end{array}$ & $\begin{array}{l}\text { New } \\
P(\mathrm{~d}) \\
\end{array}$ & $\begin{array}{c}\mathrm{CK} \\
P(\mathrm{~d})\end{array}$ & $\begin{array}{c}\left\langle H_{\mathrm{p}}\right\rangle \\
(\mathrm{mag})\end{array}$ & $\begin{array}{l}\text { Ampl. } \\
(\mathrm{mag})\end{array}$ & $\begin{array}{l}\text { Sp. } \\
\text { type }\end{array}$ \\
\hline phi Per & 10516 & 8068 & $\mathrm{U}$ & GCAS & & & & 3.986 & 0.107 & B2Vpe \\
\hline V777 Cas & 11606 & 8980 & $\mathrm{U}$ & GCAS & & & & 7.049 & 0.187 & B2Vne \\
\hline CR Cam & 21212 & 16195 & $\mathrm{U}$ & GCAS & & & & 8.397 & 0.346 & B2V:e \\
\hline CT Cam & 22298 & 16941 & U & L/IA & & & & 7.765 & 0.142 & B2Vne \\
\hline DU Eri & 28497 & 20922 & U & GCAS & & & & 5.530 & 0.084 & B2V:ne \\
\hline DX Eri & 30076 & 22024 & $\mathrm{U}$ & GCAS & & & 4.782 & 5.756 & 0.090 & $\mathrm{~B} 2 \mathrm{Ve}$ \\
\hline V1155 Tau & 32991 & 23883 & $\mathrm{U}$ & GCAS & & & & 5.871 & 0.146 & $\mathrm{~B} 2 \mathrm{Ve}$ \\
\hline V414 Aur & 33232 & 24118 & $\mathrm{U}$ & GCAS? & & 1.657 & & 8.248 & 0.107 & B2Vne \\
\hline V415 Aur & 33461 & 24238 & $\mathrm{U}$ & IA & & & & 7.836 & 0.067 & B2:V:nne \\
\hline V416 Aur & 33604 & 24326 & $\mathrm{U}$ & $\mathrm{L}$ & & & & 7.379 & 0.220 & B2V:pe \\
\hline \multirow[t]{2}{*}{ V431 Aur } & & 26354 & $\mathrm{P}$ & $?$ & 16.861 & & & 9.069 & 0.281 & $\mathrm{~B} 2 \mathrm{Ve}$ \\
\hline & 41335 & 28744 & & IA & & & & 5.220 & 0.027 & B2Vne+ \\
\hline V1390 Ori & 42908 & 29563 & $\mathrm{U}$ & IA & & & & 8.087 & 0.112 & $\mathrm{~B} 2 \mathrm{Ve}$ \\
\hline V725 Mon & 45901 & 30992 & $\mathrm{U}$ & GCAS? & & 22.222 & & 8.928 & 0.129 & $\mathrm{~B} 2 \mathrm{Ve}$ \\
\hline V728 Mon & 46380 & 31199 & U & L? & & & & 8.071 & 0.093 & B2Vne \\
\hline V733 Mon & 47761 & 31894 & U & $\mathrm{L}$ & & 249.990 & & 8.762 & 0.250 & B2V:pe \\
\hline V742 Mon & 50083 & 32947 & $\mathrm{U}$ & SPB? & & 2.369 & & 6.920 & 0.056 & $\mathrm{~B} 2 \mathrm{Ve}$ \\
\hline $\mathrm{KZ} \mathrm{CMa}$ & 50737 & 33119 & $\mathrm{U}$ & IA? & & 24.387 & & 8.721 & 0.096 & B2Vnne \\
\hline V744 Mon & 50868 & 33267 & $\mathrm{U}$ & GCAS & & & & 7.804 & 0.180 & B2Vne \\
\hline & 55439 & 34839 & $\mathrm{D}$ & IA & & & & 8.530 & 0.094 & $\mathrm{~B} 2 \mathrm{Ve}$ \\
\hline HI CMa & 55538 & 34852 & $\mathrm{U}$ & GCAS & & & & 7.826 & 0.194 & $\mathrm{~B} 2 \mathrm{Vn}(\mathrm{e})$ \\
\hline OT Gem & 58050 & 35933 & $\mathrm{U}$ & GCAS? & & 6.677 & & 6.381 & 0.209 & B2Ve \\
\hline FW CMa & 58343 & 35951 & $\mathrm{U}$ & GCAS & & & & 5.197 & 0.306 & B2Vne \\
\hline V370 Pup & 59094 & 36250 & $\mathrm{U}$ & SPB? & & 1.915 & & 8.514 & 0.115 & B2V:ne \\
\hline V373 Pup & 59497 & 36404 & U & GCAS & & & & 7.723 & 0.197 & B2V:ne \\
\hline OW Pup & 60606 & 36778 & U & GCAS & & & & 5.392 & 0.178 & B2Vne \\
\hline BT CMi & 65079 & 38855 & U & GCAS/I & & 2.183 & 2.182 & 7.750 & 0.106 & B2V(ne) \\
\hline NR Vel & 71934 & 41501 & $\mathrm{U}$ & GCAS & & & & 7.678 & 0.212 & B2V:e \\
\hline V353 Car & 97151 & 54572 & $\mathrm{U}$ & GCAS & & & & 7.701 & 0.106 & $\mathrm{~B} 2 \mathrm{Ve}$ \\
\hline DG Cru & 104722 & 58794 & $\mathrm{U}$ & GCAS & & & & 7.562 & 0.236 & B2Vne \\
\hline V955 Cen & 114800 & 64578 & $\mathrm{U}$ & SPB? & & 6.491 & & 7.984 & 0.109 & B2Vpe \\
\hline V1010 Cen & 127489 & 71264 & $\mathrm{U}$ & GCAS & & & & 9.098 & 0.308 & B2Vne \\
\hline V364 Nor & 144320 & 79038 & $\mathrm{P}$ & SPB & 2.863 & & & 9.301 & 0.110 & $\mathrm{~B} 2 \mathrm{Ve}$ \\
\hline chi Oph & 148184 & 80569 & $\mathrm{U}$ & GCAS & & & & 4.293 & 0.136 & B2Vne \\
\hline OZ Nor & 148259 & 80721 & $\mathrm{U}$ & GCAS & & & & 7.358 & 0.265 & $\mathrm{~B} 2 \mathrm{Ve}$ \\
\hline V1078 Sco & 156468 & 84745 & $\mathrm{U}$ & GCAS? & & 1.966 & & 7.902 & 0.196 & B2V:ne \\
\hline alf Ara & 158427 & 85792 & U & GCAS & & & & 2.780 & 0.087 & B2Vne \\
\hline V1083 Sco & 159684 & 86253 & U & GCAS/S & & 1.292 & & 8.256 & 0.212 & B2Vne \\
\hline V2048 Oph & 164284 & 88149 & $\mathrm{U}$ & GCAS? & & 4.695 & & 4.717 & 0.180 & $\mathrm{~B} 2 \mathrm{Ve}$ \\
\hline V4031 Sgr & 170235 & 90610 & $\mathrm{U}$ & GCAS & & & & 6.621 & 0.113 & B2Vnne \\
\hline V4400 Sgr & 171348 & 91130 & $\mathrm{U}$ & GCAS & & & & 8.091 & 0.106 & B2Vnne \\
\hline V2119 Cyg & 194335 & 100574 & $\mathrm{U}$ & GCAS & & & & 5.803 & 0.095 & B2Vne \\
\hline V2120 Cyg & 194883 & 100744 & $\mathrm{U}$ & GCAS & & & & 7.260 & 0.208 & $\mathrm{~B} 2 \mathrm{Ve}$ \\
\hline V420 Cep & 239618 & 104883 & $\mathrm{U}$ & GCAS? & & & 1.709 & 8.733 & 0.279 & $\mathrm{~B} 2 \mathrm{Ve}$ \\
\hline ups Cyg & 202904 & 105138 & $\mathrm{U}$ & GCAS & & & & 4.334 & 0.283 & B2Vne \\
\hline V2162 Cyg & 204722 & 106079 & $\mathrm{U}$ & GCAS & & & & 7.619 & 0.102 & B2V:nne: \\
\hline V2166 Cyg & 205618 & 106620 & $\mathrm{U}$ & GCAS & & & & 8.155 & 0.102 & B2Vne \\
\hline V818 Cas & 223501 & 117514 & $\mathrm{U}$ & GCAS & & & & 7.744 & 0.122 & B2Vn(e) \\
\hline MX Pup & 68980 & 40274 & $\mathrm{U}$ & $\mathrm{L}$ & & & & 4.721 & 0.230 & B2ne \\
\hline HR Car & 90177 & 50843 & $\mathrm{U}$ & SDOR/L & & & & 7.599 & 0.574 & B2evar \\
\hline AG Car & 94910 & 53461 & $\mathrm{U}$ & $\mathrm{L}$ & & & & 7.261 & 1.322 & B2:pe \\
\hline BZ Cru & 110432 & 62027 & $\mathrm{U}$ & SPB? & & 1.880 & & 5.339 & 0.056 & B2pe \\
\hline P Cyg & 193237 & 100044 & $\mathrm{U}$ & $\mathrm{L}$ & & & & 4.863 & 0.172 & B2pe \\
\hline V507 Car & 90966 & 51265 & $\mathrm{U}$ & GCAS & & & & 6.418 & 0.063 & B2/B3III:ne \\
\hline V1339 Aql & 187567 & 97607 & $\mathrm{U}$ & GCAS & & & & 6.465 & 0.048 & B2.5IVe \\
\hline NT Peg & 203699 & 105623 & $\mathrm{U}$ & GCAS & & & & 6.739 & 0.145 & B2.5IVne \\
\hline BK Cam & 20336 & 15520 & $\mathrm{U}$ & GCAS & & & 0.108 & 4.697 & 0.057 & B2.5Vne \\
\hline V1150 Tau & 29441 & 21626 & $\mathrm{U}$ & L/IA & & & & 7.639 & 0.096 & B2.5Vne \\
\hline BV Cam & 32343 & 23734 & U & GCAS & & & & 5.179 & 0.140 & B $2.5 \mathrm{Ve}$ \\
\hline V695 Mon & 65875 & 39172 & $\mathrm{U}$ & GCAS? & & 22.713 & & 6.450 & 0.114 & B $2.5 \mathrm{Ve}$ \\
\hline V480 Car & 81654 & 46147 & $\mathrm{U}$ & GCAS? & & 40.036 & 39.984 & 7.892 & 0.228 & $\mathrm{~B} 2 / 3 \mathrm{~V}(\mathrm{e})$ \\
\hline & 140926 & 77452 & $\mathrm{U}$ & GCAS & & & & 7.925 & 0.068 & B2/B3Vnne \\
\hline V3984 Sgr & 163868 & 88123 & $\mathrm{U}$ & SPB? & & 4.254 & & 7.345 & 0.089 & B2/B3V:ne \\
\hline NSV 13966 & 208682 & 108226 & $\mathrm{U}$ & GCAS & & & & 5.881 & 0.048 & $\mathrm{~B} 2.5 \mathrm{Ve}$ \\
\hline ome Ori & 37490 & 26594 & $\mathrm{U}$ & GCAS & & & & 4.490 & 0.073 & B3IIIe \\
\hline QV Tel & 167128 & 89605 & U & GCAS & & & & 5.349 & 0.080 & B3IIIpe \\
\hline V382 Cep & 203467 & 105268 & $\mathrm{U}$ & GCAS & & & & 5.172 & 0.130 & B3IVe \\
\hline V434 Aur & 37657 & 26872 & $\mathrm{U}$ & $\mathrm{I} ?$ & & & & 7.252 & 0.150 & B3Vne \\
\hline V447 Aur & 40978 & 28783 & $\mathrm{U}$ & $\mathrm{I}$ ? & & 0.090 & 0.091 & 7.274 & 0.119 & $\mathrm{~B} 3 \mathrm{Ve}$ \\
\hline NSV 02977 & 45725 & 30867 & $\mathrm{U}$ & IA & & & 11.204 & 3.792 & 0.063 & B3 Ve \\
\hline V339 Pup & 49336 & 32434 & U & GCAS & & & & 6.160 & 0.072 & B3Vne \\
\hline LL CMa & 50938 & 33200 & $\mathrm{U}$ & $\mathrm{I} ?$ & & 1.913 & 1.908 & 7.577 & 0.223 & B3Ve \\
\hline V387 Pup & 62753 & 37675 & $\mathrm{U}$ & GCAS & & & & 6.527 & 0.145 & B3Vne \\
\hline & 69404 & 40397 & $\mathrm{U}$ & BCEP? & & 0.449 & 0.449 & 6.397 & 0.055 & B3Vnne \\
\hline & 72014 & 41599 & U & IA & & & & 6.543 & 0.049 & B3Vnne \\
\hline V344 Car & 75311 & 43105 & $\mathrm{U}$ & GCAS & & & & 4.439 & 0.046 & B3Vne \\
\hline OR Vel & 75465 & 43229 & $\mathrm{U}$ & IA & & & & 9.076 & 0.162 & B3Vn:e \\
\hline OU Vel & 76534 & 43792 & $\mathrm{U}$ & GCAS & & & & 8.093 & 0.069 & B3Vne \\
\hline IU Vel & 77320 & 44213 & $\mathrm{U}$ & GCAS & & & & 6.009 & 0.075 & B3Vne \\
\hline KP Mus & 100324 & 56252 & $\mathrm{U}$ & GCAS? & & & 2.279 & 8.621 & 0.119 & $\mathrm{~B} 3 \mathrm{Ve}$ \\
\hline V774 Cen & 120958 & 67809 & U & GCAS & & & & 7.567 & 0.271 & B3Vnne \\
\hline CK Cir & 128293 & 71668 & $\mathrm{U}$ & IA & & & & 6.859 & 0.087 & B3Vne \\
\hline V846 Ara & 152478 & 82868 & $\mathrm{U}$ & I/IA & & & & 6.310 & 0.089 & B3Vnpe \\
\hline V2382 Oph & 160319 & 86487 & $\mathrm{P}$ & BCEP & 0.528 & & & 7.279 & 0.055 & B3Vne \\
\hline
\end{tabular}


Table A.3. continued.

\begin{tabular}{|c|c|c|c|c|c|c|c|c|c|c|}
\hline $\begin{array}{r}\text { Var. } \\
\text { name }\end{array}$ & $\begin{array}{l}\mathrm{HD} \\
N_{\mathrm{o}} .\end{array}$ & $\begin{array}{l}\mathrm{HIP} \\
N_{\mathrm{o}} .\end{array}$ & $\begin{array}{l}\text { Var. } \\
\text { flag }\end{array}$ & $\begin{array}{l}\text { Var. } \\
\text { type }\end{array}$ & $\begin{array}{l}\text { HIP } \\
P(\mathrm{~d})\end{array}$ & $\begin{array}{l}\text { New } \\
P(\mathrm{~d})\end{array}$ & $\begin{array}{c}\text { CK } \\
P(\mathrm{~d})\end{array}$ & $\begin{array}{c}\left\langle H_{\mathrm{p}}\right\rangle \\
(\mathrm{mag})\end{array}$ & $\begin{array}{l}\text { Ampl. } \\
\text { (mag) }\end{array}$ & $\begin{array}{l}\text { Sp. } \\
\text { type }\end{array}$ \\
\hline NW Ser & 168797 & 89977 & $\mathrm{U}$ & BCEP? & & 0.439 & 0.434 & 6.133 & 0.073 & B3 Ve \\
\hline V558 Lyr & 183362 & 95673 & $\mathrm{U}$ & GCAS & & & & 6.260 & 0.075 & B3Ve \\
\hline V404 Lac & 211835 & 110177 & U & GCAS & & & & 8.374 & 0.198 & $\mathrm{~B} 3: \mathrm{Ve}$ \\
\hline V438 Aur & 38708 & 27459 & $\mathrm{U}$ & IA & & & & 8.065 & 0.104 & B3:pe:shell \\
\hline QY Gem & 51354 & 33493 & $\mathrm{U}$ & BCEP? & & 0.852 & 0.852 & 7.125 & 0.097 & B3ne \\
\hline
\end{tabular}

This table is the longer version of Table 6.

The columns are: the variable name, the HD number, the HIPPARCOS number, the HIPPARCOS variability flag, the variability type we assigned them in this study, the HIPPARCOS period (if any), the new periods found in this study ( 2 columns, for a possible second period), the periods found by Koen \& Eyer (2002) (if any), the $H p$ magnitude, the mean amplitude calculated here and the spectral type according to the Vizier database.

${ }^{a}$ The star is refered to as a EB/GS in the HIPPARCOS catalog with a period of $P=62.086 \mathrm{~d}$, but we do not see any eclipse in the folded lightcurve in this study.

${ }^{b}$ We classified as GCAS, the irregularly variable stars (no periods) of type BIII-Ve. When a star has no spectral class but only the spectral type $\mathrm{O}$ or $\mathrm{B}$, we classified it as IA. 
Table A.4. OBSG stars above the threshold $T_{\mathrm{h}}$.

\begin{tabular}{|c|c|c|c|c|c|c|c|c|c|c|c|}
\hline $\begin{array}{l}\text { Var. } \\
\text { name }\end{array}$ & $\begin{array}{l}\mathrm{HD} \\
N_{\mathrm{o}} .\end{array}$ & $\begin{array}{l}\mathrm{HIP} \\
N_{\mathrm{o}} .\end{array}$ & $\begin{array}{l}\text { Var. } \\
\text { flag }\end{array}$ & $\begin{array}{l}\text { Var. } \\
\text { type }\end{array}$ & $\begin{array}{l}\text { HIP } \\
P(\mathrm{~d})\end{array}$ & $\begin{array}{l}\text { New } \\
P(\mathrm{~d})\end{array}$ & & $\begin{array}{l}\mathrm{CK} \\
P(\mathrm{~d})\end{array}$ & $\begin{array}{c}\left\langle H_{\mathrm{p}}\right\rangle \\
(\mathrm{mag})\end{array}$ & $\begin{array}{l}\text { Ampl. } \\
\text { (mag) }\end{array}$ & $\begin{array}{l}\text { Sp. } \\
\text { type }\end{array}$ \\
\hline & 66811 & 39429 & $\bar{M}$ & IA? & & 1.880 & & & 2.136 & 0.026 & O5Iaf \\
\hline \multirow{2}{*}{ V967 Cen } & 116781 & 65637 & U & GCAS & & & & & 7.631 & 0.168 & O9/B1ab:e: \\
\hline & 206267 & 106886 & $\mathrm{U}$ & IA & & & & & 5.644 & 0.049 & O6 (f) \\
\hline NSV 08031 & 152408 & 82775 & $\mathrm{U}$ & IA & & & & & 5.829 & 0.040 & $\mathrm{O} 8 \mathrm{Iab}+\ldots$ \\
\hline & 61827 & 37334 & $\mathrm{U}$ & IA & & & & & 7.749 & 0.093 & O8/O9Ib: \\
\hline & 152424 & 82783 & U & IA & & & & & 6.384 & 0.051 & O9Ia \\
\hline NSV 06024 & 112244 & 63117 & $\mathrm{U}$ & ACYG? & & 1.845 & & 2.003 & 5.372 & 0.045 & O9Ib \\
\hline UV Aur & 34842 & 25050 & $\mathrm{P}$ & $\mathrm{M} ?$ & 394.420 & & & & 9.608 & 2.248 & O9II \\
\hline & 30614 & 22783 & $\mathrm{U}$ & L/IA? & & 1.776 & 0.885 & 0.885 & 4.295 & 0.040 & O9.5Ia SB: \\
\hline & 188209 & 97757 & $\mathrm{U}$ & ACYG? & & 5.234 & & 5.233 & 5.605 & 0.051 & O9.5Ia \\
\hline & 195592 & 101186 & $\mathrm{U}$ & ACYG? & & 1.832 & 2.414 & 0.085 & 7.215 & 0.062 & O9.5Ia \\
\hline V1074 Sco & 154368 & 83706 & $\mathrm{P}$ & ACYG & 16.106 & & & & 6.243 & 0.041 & O9.5Iab \\
\hline & 37742 & 26727 & D & IA & & & & & 1.679 & 0.029 & O9.5Ib SB \\
\hline & 154811 & 83973 & $\mathrm{U}$ & ACYG? & & 1.220 & & & 7.008 & 0.054 & O9.5Ib \\
\hline & 202124 & 104695 & $\mathrm{U}$ & IA & & & & & 7.809 & 0.060 & $\mathrm{O} 9.5 \mathrm{Ib}$ \\
\hline & 209975 & 109017 & M & ACYG? & & 1.264 & & & 5.121 & 0.028 & O9.5Ib \\
\hline V689 Mon & 47432 & 31766 & U & ACYG? & & 3.548 & 6.359 & 3.549 & 6.263 & 0.053 & O9.5II \\
\hline the Mus & 113904 & 64094 & U & ACYG? & & 1.748 & & & 5.482 & 0.037 & O9.5II \\
\hline V2076 Oph & 160641 & 86605 & $\mathrm{U}$ & IA & & & & & 9.856 & 0.148 & O9.5-B1Ia(p) \\
\hline eps Ori & 37128 & 26311 & $\mathrm{U}$ & IA & & & & & 1.623 & 0.041 & B0Ia \\
\hline & 91969 & 51866 & $\mathrm{U}$ & ACYG? & & & & 3.930 & 6.513 & 0.056 & B0Ia \\
\hline NSV 06544 & 122879 & 68902 & U & IA & & & & & 6.457 & 0.047 & B0Ia \\
\hline mu. Nor & 149038 & 81122 & $\mathrm{U}$ & IA & & & & & 4.919 & 0.029 & B0Ia \\
\hline V4398 Sgr & 171012 & 90950 & $\mathrm{P}$ & $\mathrm{ACYG}$ & 2.420 & & & & 6.912 & 0.057 & B0Ia/ab \\
\hline GS Mus & 105056 & 58998 & $\mathrm{U}$ & ACYG? & & & & 3.853 & 7.392 & 0.085 & B0Iab:pe \\
\hline & 150898 & 82171 & $\mathrm{U}$ & IA & & & & & 5.548 & 0.038 & B0Iab \\
\hline & & 3197 & & IA & & & & & 9.334 & 0.105 & B0Ib \\
\hline V526 Car & 94909 & 53479 & $\mathrm{U}$ & ACYG? & & 16.972 & & & 7.413 & 0.071 & BOIb \\
\hline V1357 Cyg & 226868 & 98298 & $\mathrm{U}$ & ACYG? & & 2.797 & & 2.798 & 8.987 & 0.094 & B0Ib \\
\hline V2157 Cyg & 204172 & 105811 & $\mathrm{U}$ & ACYG? & & 2.020 & 3.049 & 3.048 & 5.890 & 0.045 & BOIb \\
\hline V429 Cep & 205196 & 106285 & $\mathrm{U}$ & ACYG? & & 1.056 & & & 7.513 & 0.054 & B0Ib \\
\hline NSV 04330 & 76968 & 43989 & $\mathrm{U}$ & $?$ & & 5.751 & & 5.750 & 7.127 & 0.061 & B0II \\
\hline & & 114990 & $\mathrm{U}$ & $\mathrm{L}$ & & & & & 8.589 & 0.083 & B0II \\
\hline & 18076 & 13736 & $\mathrm{U}$ & IA? & & 1.438 & & 0.736 & 9.198 & 0.098 & B0II-III \\
\hline NSV 02641 & 38771 & 27366 & U & IA & & & & & 2.004 & 0.038 & B0.5Iavar \\
\hline DW Cru & 112272 & 63170 & $\mathrm{U}$ & IA & & & & & 7.479 & 0.088 & B0.5Ia \\
\hline NSV 06193 & 115842 & 65129 & $\mathrm{U}$ & ACYG? & & 10.309 & & 13.382 & 6.089 & 0.066 & B0.5Ia \\
\hline NSV 07343 & 142468 & 78145 & $\mathrm{U}$ & ACYG? & & 2.209 & & & 8.032 & 0.069 & B0.5Ia \\
\hline & 152234 & 82676 & U & IA & & & & & 5.517 & 0.039 & B0.5Ia \\
\hline & 167264 & 89439 & $\mathrm{U}$ & IA & & & & 2.209 & 5.344 & 0.031 & B0/1Ia/ab \\
\hline & 194839 & 100804 & $\mathrm{U}$ & ACYG? & & 0.994 & & 3.007 & 7.624 & 0.073 & B0.5Ia \\
\hline NSV 04790 & 88879 & 50068 & $\mathrm{U}$ & IA & & & & & 8.536 & 0.077 & B0.5Iab \\
\hline & 125545 & 70228 & $\mathrm{U}$ & IA & & & & 0.087 & 7.458 & 0.057 & B0.5Iab \\
\hline V4383 Sgr & 166443 & 89129 & $\mathrm{U}$ & IA & & & & & 8.739 & 0.103 & B0.5Iab:ne \\
\hline V342 Vel & 89767 & 50598 & U & ACYG? & & 1.154 & & 0.090 & 7.244 & 0.057 & B0.5Ib \\
\hline V513 Car & 91943 & 51857 & $\mathrm{P}$ & ACYG & 6.445 & & & & 6.739 & 0.049 & B0.5Ib \\
\hline & 93619 & 52762 & $\mathrm{U}$ & IA & & & & 1.830 & 7.010 & 0.059 & B0.5Ib \\
\hline & 213087 & 110817 & $\mathrm{U}$ & IA & & & & & 5.597 & 0.046 & B0.5Ib \\
\hline & 190336 & 98740 & $\mathrm{U}$ & IA? & & & & 0.224 & 8.642 & 0.101 & B0.7II-III \\
\hline kap Cas & 2905 & 2599 & $\mathrm{U}$ & ACYG? & & 2.646 & & 2.647 & 4.213 & 0.068 & B1Ia \\
\hline V786 Cas & 13256 & 10243 & $\mathrm{U}$ & ACYG? & & 1.976 & & 2.954 & 8.725 & 0.094 & B1Ia \\
\hline & 58510 & 35997 & $\mathrm{U}$ & IA & & & & & 6.824 & 0.064 & B1Ia \\
\hline V499 Car & 89201 & 50272 & $\mathrm{U}$ & BCEP? & & 0.082 & 1.813 & & 7.952 & 0.068 & B1Ia \\
\hline KY Mus & 109867 & 61703 & $\mathrm{U}$ & ACYG? & & 4.482 & 1.825 & 4.483 & 6.270 & 0.058 & B1Ia \\
\hline & 113422 & 63802 & U & IA & & & & & 8.368 & 0.079 & B1Ia \\
\hline & 114340 & 64324 & $\mathrm{U}$ & ACYG? & & 4.425 & & 7.186 & 8.143 & 0.080 & B1Ia comp \\
\hline & 115363 & 64896 & $\mathrm{U}$ & ACYG? & & 5.119 & & 5.118 & 7.905 & 0.088 & B1Ia comp \\
\hline V1058 Sco & 148688 & 80945 & $\mathrm{P}$ & ACYG & 6.331 & & & & 5.385 & 0.062 & B1Ia \\
\hline V900 Sco & 152235 & 82669 & $\mathrm{U}$ & ACYG? & & 4.149 & & 4.150 & 6.415 & 0.084 & B1Ia \\
\hline zet01 Sco & 152236 & 82671 & U & SDOR/L & & & & & 4.836 & 0.076 & B1Iae \\
\hline V430 Sct & 169454 & 90281 & U & ACYG? & & & & 9.224 & 6.725 & 0.083 & B1Ia \\
\hline V449 Sct & 170938 & 90907 & $\mathrm{U}$ & ACYG? & & 2.273 & & & 7.964 & 0.086 & B1Ia \\
\hline NSV 14337 & 216411 & 112881 & $\mathrm{U}$ & IA & & & & & 7.343 & 0.070 & B1Ia \\
\hline V551 Per & 13854 & 10633 & $\mathrm{P}$ & ACYG? & 5.643 & & & & 6.557 & 0.058 & B1Iab \\
\hline V790 Cas & 15785 & 12009 & $\mathrm{U}$ & ACYG? & & 4.312 & & 4.310 & 8.478 & 0.100 & B1Iab \\
\hline V414 Car & 96248 & 54179 & $P$ & BCEP & 0.703 & & & & 6.633 & 0.067 & B1Iab \\
\hline & 108002 & 60570 & $\mathrm{U}$ & ACYG? & & 5.155 & 5.710 & & 6.965 & 0.062 & B1Iab \\
\hline & 154043 & 83603 & $\mathrm{U}$ & IA? & & 0.089 & & 0.090 & 7.196 & 0.061 & B1Iab: \\
\hline NSV 10662 & 168571 & 89946 & $\mathrm{U}$ & IA & & & & & 7.852 & 0.065 & B1Iab/b \\
\hline & 190066 & 98661 & $\mathrm{U}$ & IA & & & & & 6.556 & 0.053 & B1Iab \\
\hline & 194153 & 100484 & U & $\mathrm{L}$ & & & & & 8.633 & 0.080 & B1Iab \\
\hline V820 Cas & 224424 & 118139 & $\mathrm{U}$ & IA & & & & & 8.230 & 0.072 & B1Iab \\
\hline & 52382 & 33754 & $\mathrm{U}$ & IA & & & & & 6.543 & 0.065 & $\mathrm{~B} 1 \mathrm{Ib}$ \\
\hline NSV 04317 & 76510 & 43868 & $\mathrm{U}$ & BCEP? & & 0.632 & & & 7.975 & 0.066 & B1Ib \\
\hline rho Leo & 91316 & 51624 & $\mathrm{P}$ & ACYG & 3.427 & & & 1.492 & 3.797 & 0.032 & B1Ib SB \\
\hline NSV 05100 & 96880 & 54448 & $\mathrm{U}$ & ACYG? & & 2.473 & & 2.471 & 7.670 & 0.082 & B1Ib \\
\hline & 194057 & 100409 & $\mathrm{U}$ & IA & & & & & 7.608 & 0.060 & B1Ib \\
\hline & 54764 & 34561 & $\mathrm{U}$ & ACYG? & & 2.694 & & & 6.055 & 0.036 & B1Ib/II \\
\hline FY CMa & 58978 & 36168 & $\mathrm{U}$ & IA? & & 111.116 & 2.606 & 109.890 & 5.574 & 0.101 & B1II \\
\hline DE Cru & 104631 & 58748 & $\mathrm{P}$ & SPB & 3.688 & & & & 6.793 & 0.062 & B1II \\
\hline & 150168 & 81733 & & IA & & & & & 5.643 & 0.031 & B1II \\
\hline V4386 Sgr & 167003 & 89404 & $\mathrm{U}$ & $\mathrm{L}$ ? & & & & & 8.416 & 0.141 & $\mathrm{~B} 1 \mathrm{Ib} / \mathrm{II}$ \\
\hline bet $\mathrm{CMa}$ & 44743 & 30324 & $\mathrm{P}$ & ВСЕР & 0.251 & & & & 1.890 & 0.030 & B1II/III \\
\hline
\end{tabular}


Table A.4. continued.

\begin{tabular}{|c|c|c|c|c|c|c|c|c|c|c|c|}
\hline $\begin{array}{l}\text { Var. } \\
\text { name }\end{array}$ & $\begin{array}{l}\mathrm{HD} \\
N_{\mathrm{o}} .\end{array}$ & $\begin{array}{l}\mathrm{HIP} \\
N_{\mathrm{o}} .\end{array}$ & $\begin{array}{l}\text { Var. } \\
\text { flag }\end{array}$ & $\begin{array}{l}\text { Var. } \\
\text { type }\end{array}$ & $\begin{array}{l}\text { HIP } \\
P(\mathrm{~d})\end{array}$ & $\begin{array}{l}\text { New } \\
P(\mathrm{~d})\end{array}$ & & $\begin{array}{c}\mathrm{CK} \\
P(\mathrm{~d})\end{array}$ & $\begin{array}{c}\left\langle H_{\mathrm{p}}\right\rangle \\
(\mathrm{mag})\end{array}$ & $\begin{array}{l}\text { Ampl. } \\
\text { (mag) }\end{array}$ & $\begin{array}{l}\text { Sp. } \\
\text { type }\end{array}$ \\
\hline DL Cru & 106343 & 59678 & $\mathrm{P}$ & ACYG & 2.878 & & & & 6.261 & 0.068 & B1.5Ia \\
\hline CZ Cir & 136239 & 75224 & $\mathrm{U}$ & ACYG? & & 15.625 & & & 7.956 & 0.089 & B1.5Ia \\
\hline V361 Nor & 142758 & 78310 & $\mathrm{U}$ & IA & & & & & 7.124 & 0.050 & B1.5Ia \\
\hline QU Nor & 148379 & 80782 & $\mathrm{P}$ & $\mathrm{ACYG}$ & 4.818 & & & & 5.430 & 0.064 & B1.5Iap \\
\hline V433 Sct & & 90267 & $\mathrm{P}$ & ACYG & 6.750 & & & & 8.300 & 0.141 & B1.5Ia comp \\
\hline V1768 Cyg & 190603 & 98863 & $\mathrm{U}$ & ACYG? & & 3.497 & & 0.947 & 5.738 & 0.065 & B1.5Ia comp \\
\hline V2118 Cyg & 194279 & 100548 & $\mathrm{U}$ & ACYG? & & 2.416 & & & 7.157 & 0.075 & B1.5Ia \\
\hline \multirow[t]{2}{*}{ OS Vel } & 75860 & 43443 & $\mathrm{P}$ & ACYG & 1.938 & & & & 7.714 & 0.087 & B1.5Iab \\
\hline & 5551 & 4567 & $\mathrm{U}$ & IA & & & & & 7.863 & 0.081 & B1.5Ib \\
\hline LY CMa & 54786 & 34569 & $\mathrm{U}$ & $\mathrm{L}$ & & & & & 9.081 & 0.241 & B1/B2Ib: \\
\hline NN CMa & 58011 & 35769 & $\mathrm{U}$ & L/ACYG & & & & 21.505 & 7.146 & 0.286 & B1/B2Ib/IIn \\
\hline & 173502 & 92152 & $\mathrm{U}$ & IA & & & & & 9.706 & 0.126 & B1/B2Ib \\
\hline & 54551 & 34429 & $\mathrm{U}$ & IA & & & & & 8.608 & 0.081 & B1/B2II \\
\hline YZ Pyx & 71913 & 41586 & $\mathrm{P}$ & BCEP & 0.206 & & & & 7.643 & 0.059 & B1/B2II \\
\hline & 164002 & 88126 & $\mathrm{U}$ & SPB? & & 3.058 & 1.515 & & 7.397 & 0.062 & B1/B2II \\
\hline V4382 Sgr & 165812 & 88884 & $\mathrm{P}$ & BCEP & 0.169 & & & & 7.955 & 0.066 & B1/B2II \\
\hline V1449 Aql & 180642 & 94793 & $\mathrm{P}$ & BCEP & 0.182 & & & & 8.342 & 0.119 & B1.5II-III \\
\hline & 14143 & 10816 & $\mathrm{U}$ & L/IA? & & 0.089 & & 0.089 & 6.780 & 0.080 & B2Ia \\
\hline V554 Per & 14818 & 11279 & $\mathrm{U}$ & L/IA? & & & & & 6.324 & 0.070 & B2Ia \\
\hline V475 Per & 14956 & 11391 & $\mathrm{U}$ & ACYG? & & 1.367 & & 1.367 & 7.329 & 0.066 & B2Ia \\
\hline chi02 Ori & 41117 & 28716 & $\mathrm{P}$ & ACYG & 2.868 & & & & 4.697 & 0.057 & B2Iavar \\
\hline & 60308 & 36727 & $\mathrm{U}$ & IA? & & 0.089 & & 0.089 & 8.291 & 0.075 & B2Ia \\
\hline PV Vel & 80077 & 45467 & $\mathrm{U}$ & GCAS? & & 3.115 & & 3.116 & 7.570 & 0.151 & B2Iape \\
\hline V808 Cen & 99953 & 56050 & $\mathrm{U}$ & IA & & & & & 6.533 & 0.078 & B2Ia \\
\hline & 119646 & 67206 & $\mathrm{U}$ & ACYG? & & 5.682 & & 8.962 & 6.645 & 0.044 & B2Ia \\
\hline CX Cir & 134959 & 74660 & $\mathrm{U}$ & $\mathrm{L}$ & & & & & 8.108 & 0.170 & B2Ia \\
\hline V2174 Cyg & 235679 & 108476 & $\mathrm{U}$ & ACYG? & & 1.062 & & & 8.979 & 0.139 & B2Ia: \\
\hline V1073 Sco & 154090 & 83574 & $\mathrm{U}$ & ACYG? & & 1.593 & & 1.593 & 4.915 & 0.063 & B2Iab \\
\hline & 10898 & 8415 & $\mathrm{U}$ & ACYG? & & 1.927 & 2.857 & 1.927 & 7.502 & 0.056 & $\mathrm{~B} 2 \mathrm{Ib}$ \\
\hline V963 Ori & 43837 & 30041 & $\mathrm{U}$ & BCEP $^{a}$ & & 2.584 & & 5.926 & 8.574 & 0.085 & B2Ibp: \\
\hline & 172694 & 91725 & $\mathrm{U}$ & AYCG? & & 9.263 & & & 8.242 & 0.074 & B2Ibe \\
\hline NSV 13041 & 193946 & 100391 & $\mathrm{U}$ & ACYG? & & 24.391 & & & 9.088 & 0.115 & B2Ib \\
\hline V337 Cep & 206165 & 106801 & $\mathrm{U}$ & $\mathrm{L}$ & & & & & 4.844 & 0.056 & B2Ib \\
\hline & 33203 & 24072 & $\mathrm{R}$ & IA & & & & & 6.059 & 0.033 & B2II: comp \\
\hline PT Pup & 61068 & 37036 & $\mathrm{U}$ & $\mathrm{BCEP}^{b}$ & & 1.051 & & 0.181 & 5.651 & 0.032 & B2II \\
\hline & 60993 & 37040 & $\mathrm{U}$ & $\mathrm{L}$ ? & & 9.095 & 0.090 & & 8.775 & 0.116 & B2II \\
\hline & 62483 & 37471 & $\mathrm{U}$ & IA? & & 3.905 & & & 8.213 & 0.081 & B2II \\
\hline V360 Nor & 141318 & 77645 & $\mathrm{P}$ & SPB & 1.467 & & & & 5.783 & 0.034 & B2II \\
\hline & 151158 & 82199 & $\mathrm{U}$ & IA & & & & & 8.237 & 0.070 & B2Ib/II \\
\hline & 160430 & 86515 & D & IA & & & & & 7.971 & 0.453 & B2II \\
\hline KK Vel & 78616 & 44790 & $\mathrm{P}$ & BCEP & 0.216 & & & & 6.781 & 0.068 & B2II/III \\
\hline lam Pav & 173948 & 92609 & $\mathrm{U}$ & IA & & & & 6.181 & 4.168 & 0.089 & B2II-III \\
\hline V519 Car & 92964 & 52405 & $\mathrm{U}$ & ACYG? & & 14.711 & & 14.680 & 5.444 & 0.079 & B2.5Ia \\
\hline PU Gem & 42087 & 29225 & $\mathrm{P}$ & ACYG & 6.807 & & & & 5.800 & 0.057 & B2.5Ib \\
\hline V536 Car & 98410 & 55207 & $P$ & ACYG & 1.453 & & & & 8.910 & 0.162 & B2/B3Ib/II \\
\hline & 116084 & 65247 & $\mathrm{U}$ & IA & & & & & 5.870 & 0.052 & B2.5Ib \\
\hline & 46185 & 31088 & $\mathrm{U}$ & $?$ & & 2.131 & & & 6.740 & 0.040 & B2/B3II: \\
\hline V639 Cas & 225094 & 274 & $\mathrm{U}$ & ACYG? & & 2.880 & 1.348 & 2.881 & 6.336 & 0.072 & B3Ia \\
\hline V520 Per & 14134 & 10805 & $\mathrm{U}$ & L/IA? & & & & 1.625 & 6.699 & 0.093 & B3Ia \\
\hline PX Gem & 43384 & 29840 & $\mathrm{P}$ & ACYG & 13.700 & & & & 6.364 & 0.076 & B3Ia \\
\hline omi02 CMa & 53138 & 33977 & $P$ & ACYG & 24.440 & & & & 3.008 & 0.076 & B3Ia \\
\hline OP Vel & 75149 & 43082 & $\mathrm{U}$ & SPB? & & 1.086 & & 1.086 & 5.520 & 0.051 & B3Ia \\
\hline V504 Car & 90706 & 51150 & $\mathrm{U}$ & ACYG? & & 1.600 & & 1.601 & 7.171 & 0.070 & B3Ia \\
\hline V4384 Sgr & 166628 & 89203 & $\mathrm{U}$ & ACYG? & & 1.404 & & & 7.264 & 0.079 & B3Ia/Iab \\
\hline NSV 10498 & 167838 & 89641 & U & IA & & & & & 6.812 & 0.052 & B3Ia/Iab \\
\hline V1403 Aql & 178129 & 93904 & $\mathrm{U}$ & ACYG? & & 5.917 & & 5.912 & 7.539 & 0.080 & B3Ia \\
\hline V1661 Cyg & 198478 & 102724 & $\mathrm{U}$ & $\mathrm{L}$ & & & & 4.885 & 4.922 & 0.053 & B3Ia \\
\hline & 224055 & 117884 & $\mathrm{U}$ & $\mathrm{L}$ & & & & & 7.343 & 0.084 & B3Ia \\
\hline & 7103 & 5649 & $\mathrm{U}$ & IA & & & & & 8.472 & 0.086 & B3Ib \\
\hline & 250290 & 28513 & $\mathrm{U}$ & IA & & & & 109.529 & 7.493 & 0.055 & B3Ib \\
\hline BU Cru & 111934 & 62913 & $\mathrm{U}$ & ACYG? & & 56.956 & & 57.110 & 6.977 & 0.160 & B3Ib: \\
\hline & 111990 & 62953 & $\mathrm{U}$ & ACYG? & & 2.890 & & 2.891 & 6.853 & 0.063 & B3Ib \\
\hline V449 Cep & 239923 & 110200 & $\mathrm{U}$ & ACYG? & & 1.220 & & & 9.009 & 0.103 & B3Ib \\
\hline V425 Pup & 68535 & 40139 & $\mathrm{U}$ & $\mathrm{L}$ & & & & 4.211 & 7.898 & 0.143 & B3II \\
\hline & 194779 & 100771 & $\mathrm{U}$ & $\mathrm{L}$ ? & & & & & 7.837 & 0.090 & B3II \\
\hline V448 Cep & & 109606 & $\mathrm{U}$ & SPB? & & 20 & 2.890 & 211.862 & 9.658 & 0.150 & B3II: \\
\hline V715 Mon & 49567 & 32682 & $\mathrm{U}$ & IA? & & 4.348 & & 2.559 & 6.116 & 0.048 & B3II-III \\
\hline & 54104 & 34355 & $\mathrm{U}$ & SPB? & & 1.261 & & 1.671 & 8.394 & 0.129 & B3II-III \\
\hline V4030 Sgr & 168625 & 89963 & $\mathrm{U}$ & $\mathrm{L}$ & & & & & 8.475 & 0.135 & B2/5Ia(e) \\
\hline V975 Sco & 157038 & 85020 & $\mathrm{U}$ & IA & & & & & 6.446 & 0.074 & $\mathrm{~B} 4 \mathrm{Ia}+\ldots$ \\
\hline Fg Sge & & 99527 & $\mathrm{U}$ & $\mathrm{L}$ & & & & 0.089 & 9.351 & 0.452 & B4Ieq-K2Ib \\
\hline & 62150 & 37444 & $\mathrm{U}$ & ACYG? & & 1.534 & & 0.798 & 7.783 & 0.077 & B4Iab \\
\hline & 4841 & 4006 & $\mathrm{U}$ & IA & & & & 12.912 & 7.004 & 0.074 & B5Ia \\
\hline & 13267 & 10227 & $\mathrm{U}$ & ACYG? & & 2.653 & & 2.654 & 6.466 & 0.062 & B5Ia \\
\hline eta $\mathrm{CMa}$ & 58350 & 35904 & $\mathrm{U}$ & $\mathrm{L}$ & & & & 4.704 & 2.422 & 0.093 & B5Ia \\
\hline GX Vel & 79186 & 45085 & $\mathrm{U}$ & IA & & & & & 5.055 & 0.071 & B5Ia \\
\hline V922 Cen & 102997 & 57808 & U & ACYG? & & 2.977 & & 2.980 & 6.608 & 0.065 & B5Ia \\
\hline & 111973 & 62931 & $\mathrm{U}$ & ACYG? & & 9.536 & & 9.536 & 5.967 & 0.065 & B5Ia \\
\hline V440 Cep & 239828 & 108714 & $\mathrm{U}$ & $\mathrm{L}$ & & & & 2.318 & 9.475 & 0.123 & B5Ia \\
\hline & 9105 & 7088 & & ACYG? & & 1.866 & & & 7.627 & 0.056 & B5Iab \\
\hline & 36371 & 25984 & $\mathrm{U}$ & $\mathrm{L}$ ? & & & & & 4.801 & 0.053 & B5Iab \\
\hline NSV 03231 & 49888 & 32786 & $\mathrm{U}$ & IA & & & & & 7.151 & 0.069 & B5Iab/b \\
\hline LN Vel & 74371 & 42679 & $\mathrm{U}$ & IA & & & & 8.291 & 5.269 & 0.073 & B5Iab \\
\hline V523 Car & 94304 & 53109 & $\mathrm{P}$ & ACYG & 3.296 & & & & 6.972 & 0.063 & B5Iab \\
\hline
\end{tabular}


Table A.4. continued.

\begin{tabular}{|c|c|c|c|c|c|c|c|c|c|c|c|}
\hline $\begin{array}{r}\text { Var. } \\
\text { name }\end{array}$ & $\begin{array}{l}\mathrm{HD} \\
N_{\mathrm{o}} .\end{array}$ & $\begin{array}{l}\mathrm{HIP} \\
N_{\mathrm{o}} .\end{array}$ & $\begin{array}{l}\text { Var. } \\
\text { flag }\end{array}$ & $\begin{array}{l}\text { Var. } \\
\text { type }\end{array}$ & $\begin{array}{l}\text { HIP } \\
P(\mathrm{~d})\end{array}$ & $\begin{array}{l}\text { New } \\
P(\mathrm{~d}) \\
\end{array}$ & & $\begin{array}{c}\mathrm{CK} \\
P(\mathrm{~d}) \\
\end{array}$ & $\begin{array}{c}\left\langle H_{\mathrm{p}}\right\rangle \\
(\mathrm{mag})\end{array}$ & $\begin{array}{l}\text { Ampl. } \\
\text { (mag) }\end{array}$ & $\begin{array}{l}\text { Sp. } \\
\text { type }\end{array}$ \\
\hline \multirow[t]{2}{*}{ V406 Lac } & 212455 & 110500 & $\mathrm{U}$ & IA & & & & & 7.983 & 0.068 & B5Iab \\
\hline & 4768 & 3932 & & IA & & & & & 7.672 & 0.062 & B5Ib \\
\hline NSV 00542 & 9311 & 7232 & $\mathrm{U}$ & ACYG? & & 58.823 & & 58.651 & 7.351 & 0.081 & B5Ib \\
\hline MU CMa & 56847 & 35355 & $\mathrm{U}$ & ACYG? & & 0.094 & 2.698 & 0.094 & 8.987 & 0.107 & B5Ib \\
\hline NSV 05072 & 95880 & 53996 & U & ACYG? & & 2.933 & & & 7.041 & 0.066 & B5Ib \\
\hline NP CMa & 58200 & 35829 & $\mathrm{U}$ & IA & & & & & 9.056 & 0.160 & B5Ib/II \\
\hline V782 Cas & 12882 & 9997 & $\mathrm{U}$ & ACYG? & & 47.621 & & & 7.698 & 0.081 & B6Ia \\
\hline \multirow[t]{2}{*}{ V425 Per } & 15497 & 11769 & $\mathrm{U}$ & $\mathrm{ACYG}^{c}$ & & 5.780 & & 5.783 & 7.175 & 0.059 & B6Ia \\
\hline & 25914 & 19404 & $\mathrm{U}$ & IA & & & & & 8.161 & 0.077 & B6Ia \\
\hline NSV 00466 & 7902 & 6229 & $\mathrm{U}$ & ACYG? & & 5.405 & & & 7.079 & 0.059 & B6Ib \\
\hline V369 Car & 91619 & 51676 & U & IA? & & & & 1.115 & 6.201 & 0.053 & B7Ia \\
\hline HT Sge & 183143 & 95657 & $\mathrm{U}$ & $\mathrm{L}$ & & & & & 6.956 & 0.122 & B7Ia \\
\hline V1507 Cyg & 187399 & 97472 & $\mathrm{P}$ & ACYG & 27.971 & & & & 7.054 & 0.111 & B7Ia:e \\
\hline \multirow[t]{2}{*}{ LR Vel } & 80558 & 45675 & $\mathrm{U}$ & ACYG? & & 1.695 & & 1.695 & 5.977 & 0.072 & B7Iab \\
\hline & 60369 & 36706 & $\mathrm{U}$ & IA & & & & & 8.147 & 0.097 & B7Ib \\
\hline \multirow[t]{2}{*}{ NSV 00806} & 14542 & 11115 & U & ACYG? & & 31.250 & & & 7.128 & 0.060 & B8Ia \\
\hline & 17145 & 13022 & $\mathrm{U}$ & IA & & & & & 8.269 & 0.075 & B8Ia \\
\hline \multirow[t]{3}{*}{ bet Ori } & 34085 & 24436 & $\mathrm{P}$ & ACYG & 2.075 & & & & 0.193 & 0.039 & B8Ia \\
\hline & 105071 & 59003 & $\mathrm{U}$ & ACYG? & & 2.490 & & 1.454 & 6.354 & 0.054 & B8Ia-Iab \\
\hline & 111558 & 62722 & $\mathrm{U}$ & IA & & & & & 7.280 & 0.051 & B8Ia \\
\hline \multirow[t]{3}{*}{ FX Lib } & 142983 & 78207 & U & IA & & & & & 4.935 & 0.049 & B8Ia/Iab \\
\hline & 184943 & 96397 & $\mathrm{U}$ & $\mathrm{L}$ ? & & & & & 8.318 & 0.082 & B8Ia \\
\hline & 186745 & 97209 & $\mathrm{U}$ & IA & & & & & 7.166 & 0.059 & B8Ia \\
\hline \multirow{2}{*}{ V2140 Cyg } & 199478 & 103312 & U & L? & & & & & 5.799 & 0.075 & B8Ia \\
\hline & 15620 & 11841 & $\mathrm{U}$ & IA & & & & & 8.497 & 0.078 & B8Iab \\
\hline \multirow[t]{7}{*}{ V508 Car } & 91024 & 51310 & $\mathrm{P}$ & ACYG & 2.399 & & & & 7.684 & 0.062 & B8Iab \\
\hline & & 66904 & M & BCEP? & & 0.461 & & & 6.928 & 0.042 & B8Iab: \\
\hline & 155416 & 84238 & $\mathrm{U}$ & ACYG? & & 11.650 & & & 6.674 & 0.048 & B8Iab-Ib \\
\hline & 14322 & 10926 & $\mathrm{U}$ & ACYG? & & 0.895 & & 6.467 & 6.863 & 0.066 & B8Ib \\
\hline & 17857 & 13608 & $\mathrm{U}$ & IA & & & & 94.607 & 7.857 & 0.070 & B8Ib \\
\hline & 20567 & 15698 & $\mathrm{U}$ & IA & & & & & 8.676 & 0.085 & B8Ib \\
\hline & 204710 & 106071 & $\mathrm{U}$ & IA & & & & & 7.004 & 0.047 & B8Ib \\
\hline NSV 13963 & 208501 & 108165 & $\mathrm{U}$ & ACYG? & & 1.410 & & & 5.902 & 0.060 & B8Ibvar \\
\hline NN Vel & 68161 & 39919 & $\mathrm{P}$ & ACYG & 17.028 & & & & 5.635 & 0.034 & $\mathrm{~B} 8 \mathrm{Ib} / \mathrm{II}$ \\
\hline
\end{tabular}

This table is the appendixed version Table 7.

The columns are: the variable name, the HD number, the HIPPARCOS number, the HIPPARCOS variability flag, the variability type we assigned them in this study, the HIPPARCOS period (if any), the new periods found in this study ( 2 columns, for a possible second period), the periods found by Koen \& Eyer (2002) (if any), the $H p$ magnitude, the mean amplitude calculated here and the spectral type according to the Vizier database.

ACYG type was attributed to variable stars of spectral class Ia-Iab-Ib, and some I/II.

${ }^{a}$ HIP30041 is reported to have a period $P=0.2 \mathrm{~d}$.

${ }^{b}$ HIP37036 is reported to have a period $P=0.162$ d.

${ }^{c} \mathrm{HIP} 11769$ is reported to have a period $P=16.1 \mathrm{~d}$. 


\section{Appendix B: Lightcurves of variable OB stars from the Hipparcos catalog}

Above each lightcurve are found the HIPPARCOS number the $H_{\mathrm{p}}$ magnitude, the mean amplitude (derived from this study) the phased period, and the spectral type.

\section{B.1. Catalog of eclipsing binaries}
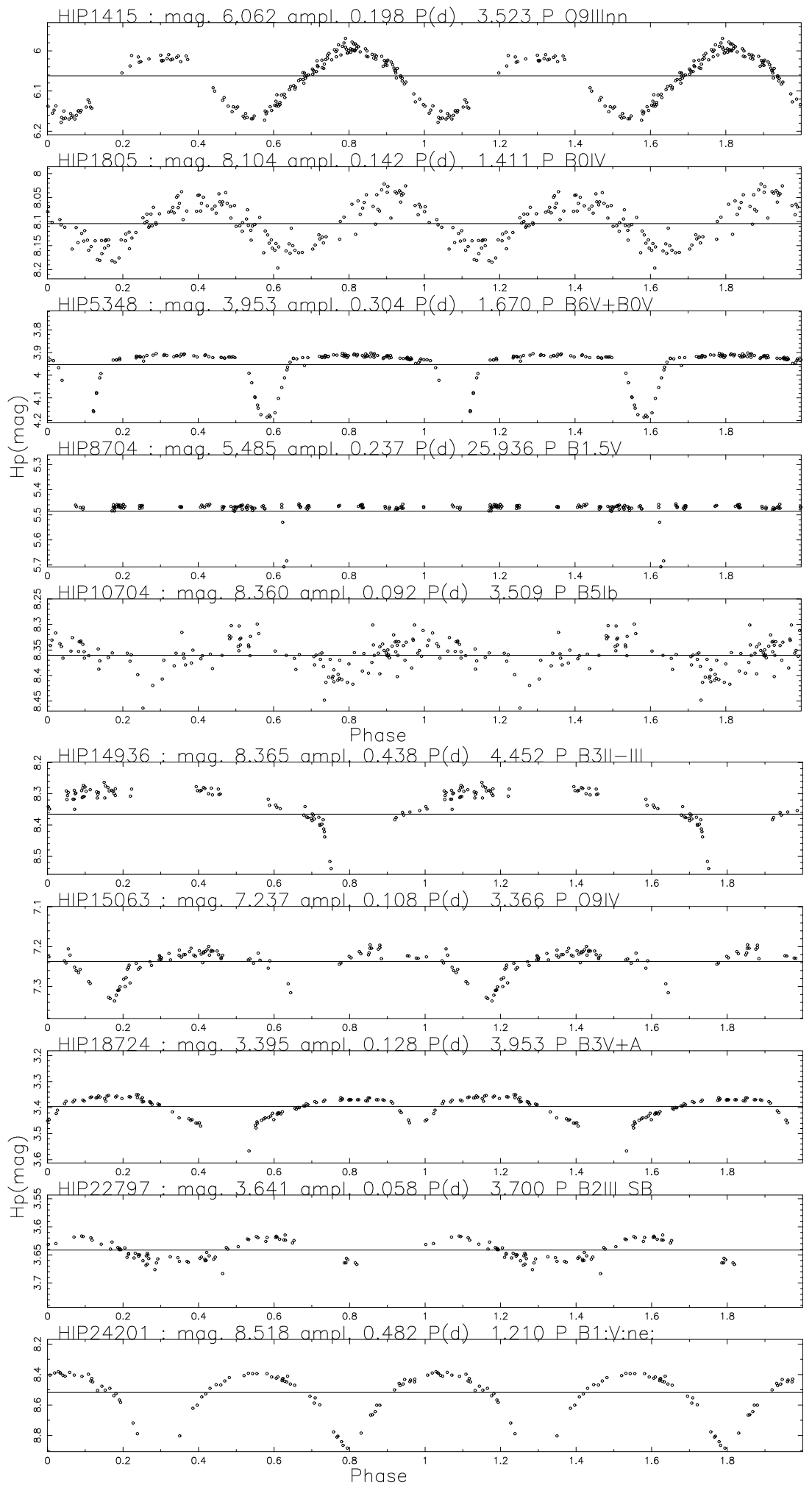

Fig. B.1. This figure plots $H p$ mag. vs. Phase. Epoch is arbitrarily JD-2 $440000=7800$, which is the beginning of the HIPPARCOS observations. The $H p$ magnitude, mean amplitude and periods are accurate to $\sim 0.1 \%$. 

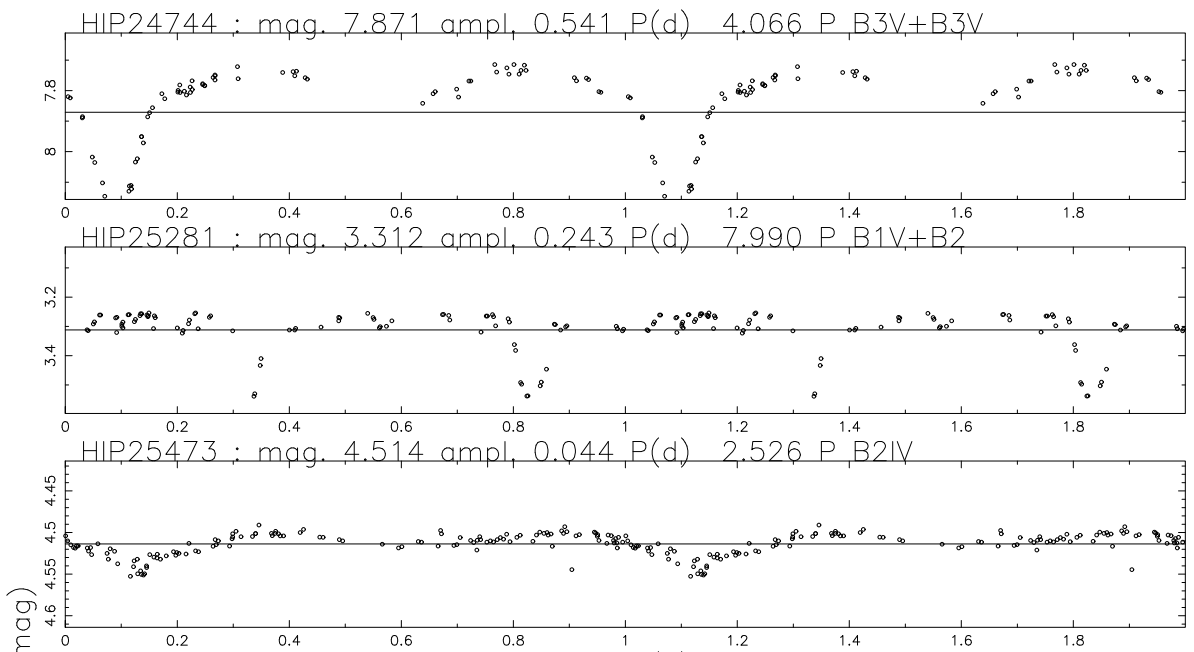

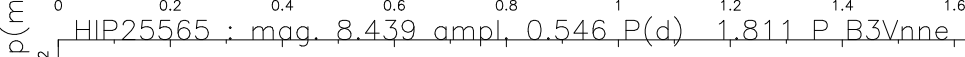
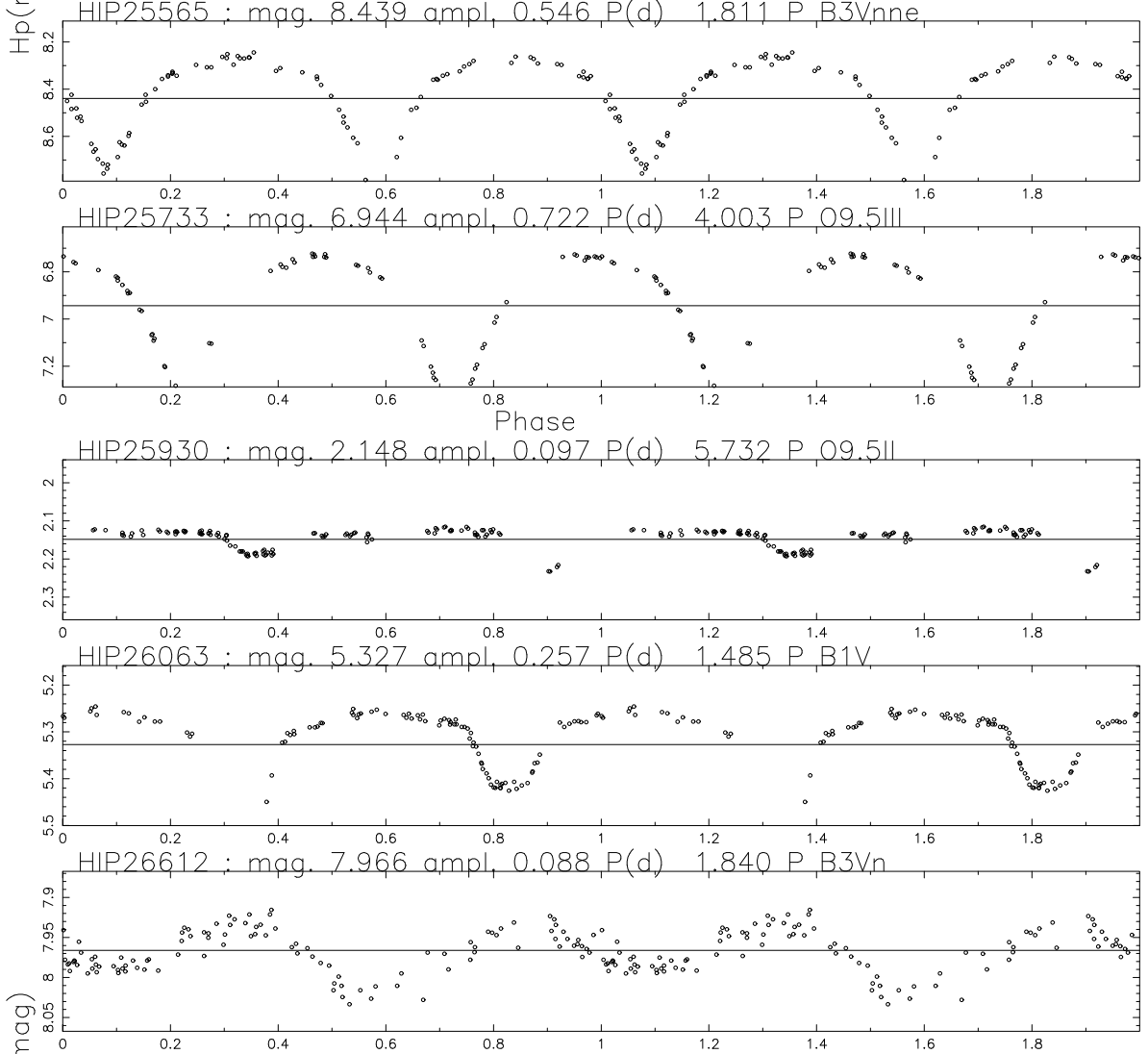

E HIP, 281,42: mag. $7.203 \mathrm{ampl}^{0.2} 0.233^{1} \mathrm{P}(\mathrm{d}) \quad 6.572^{0.4} \mathrm{P}_{1}^{1.4} \mathrm{~B} 2 \mathrm{~V}$
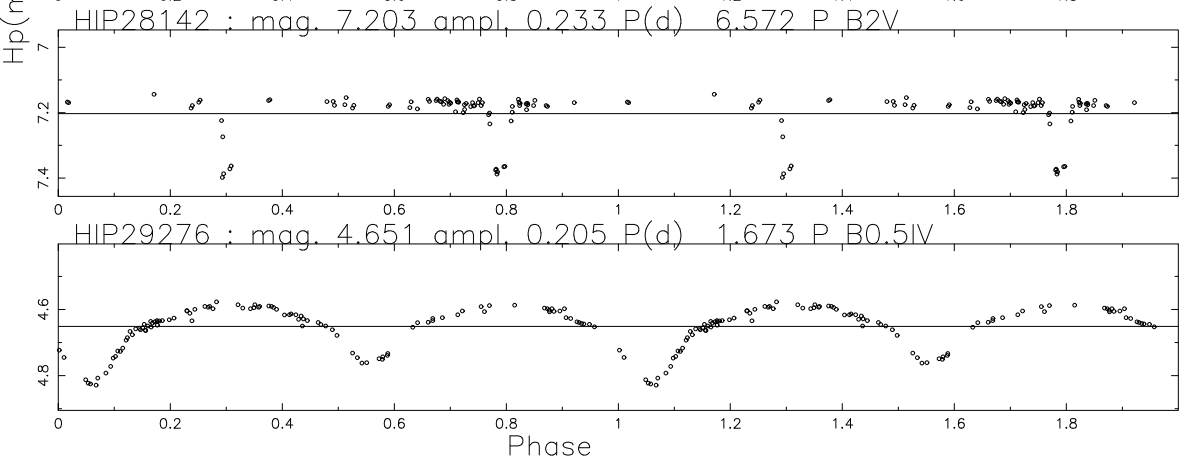

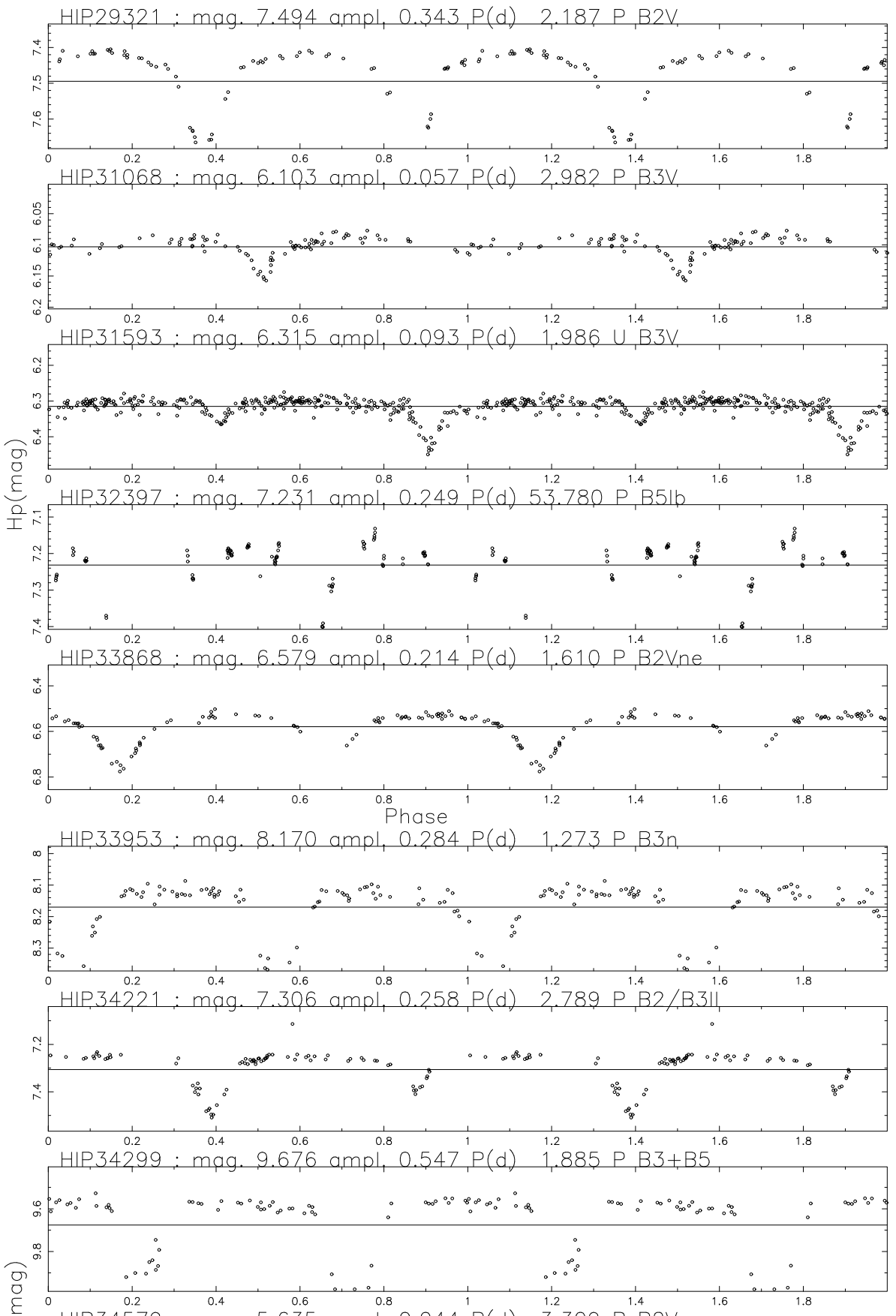

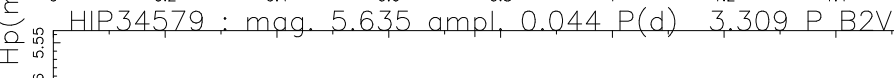

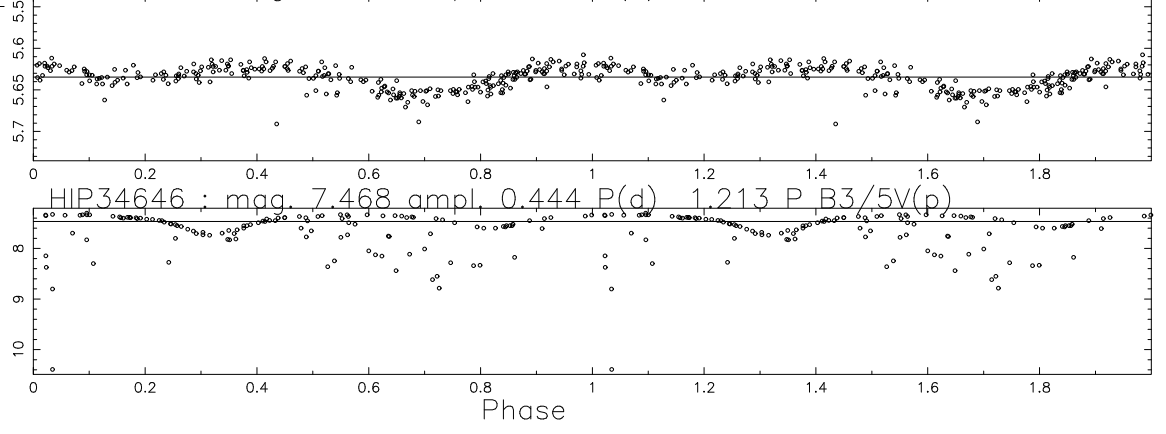



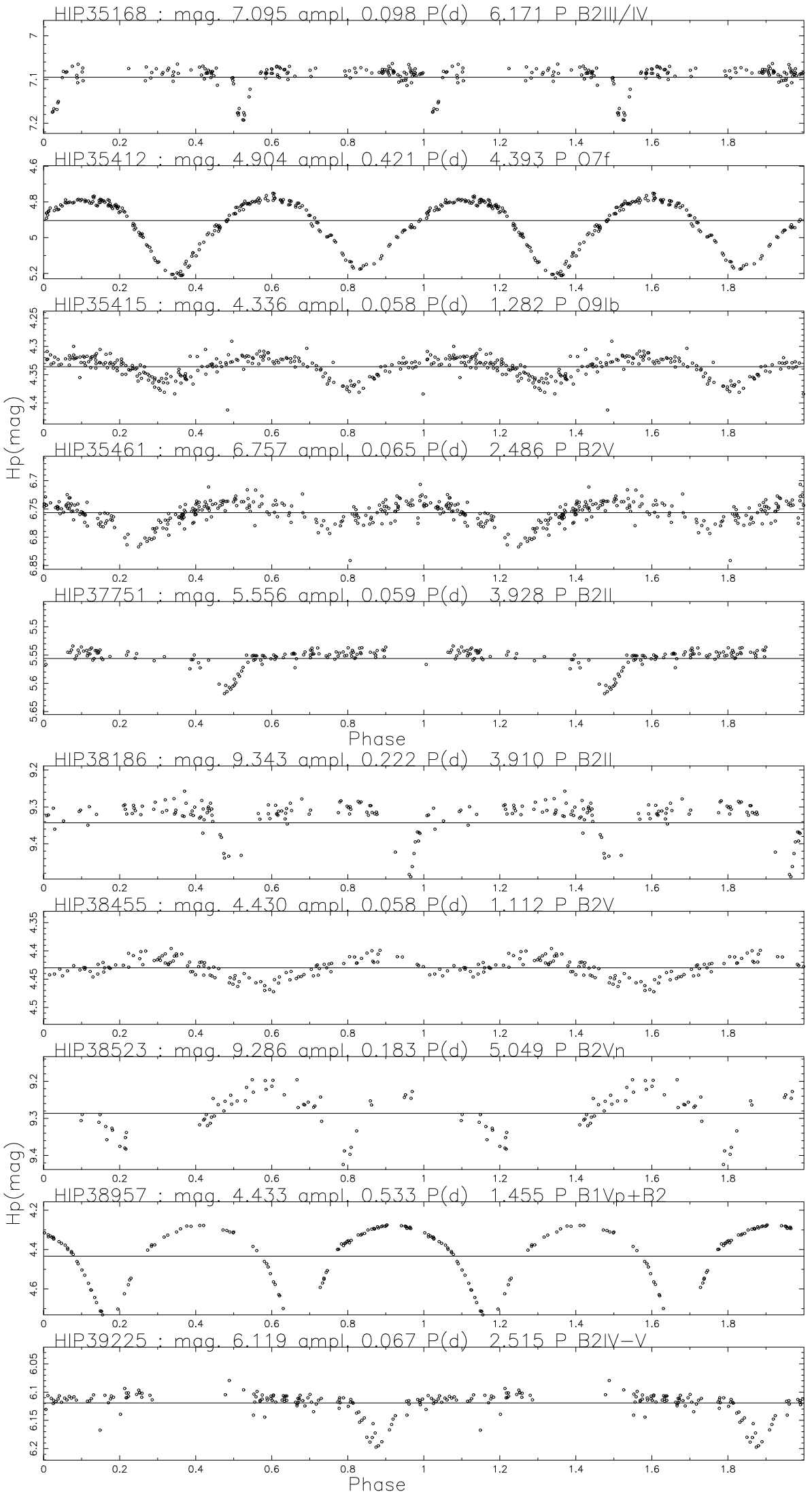

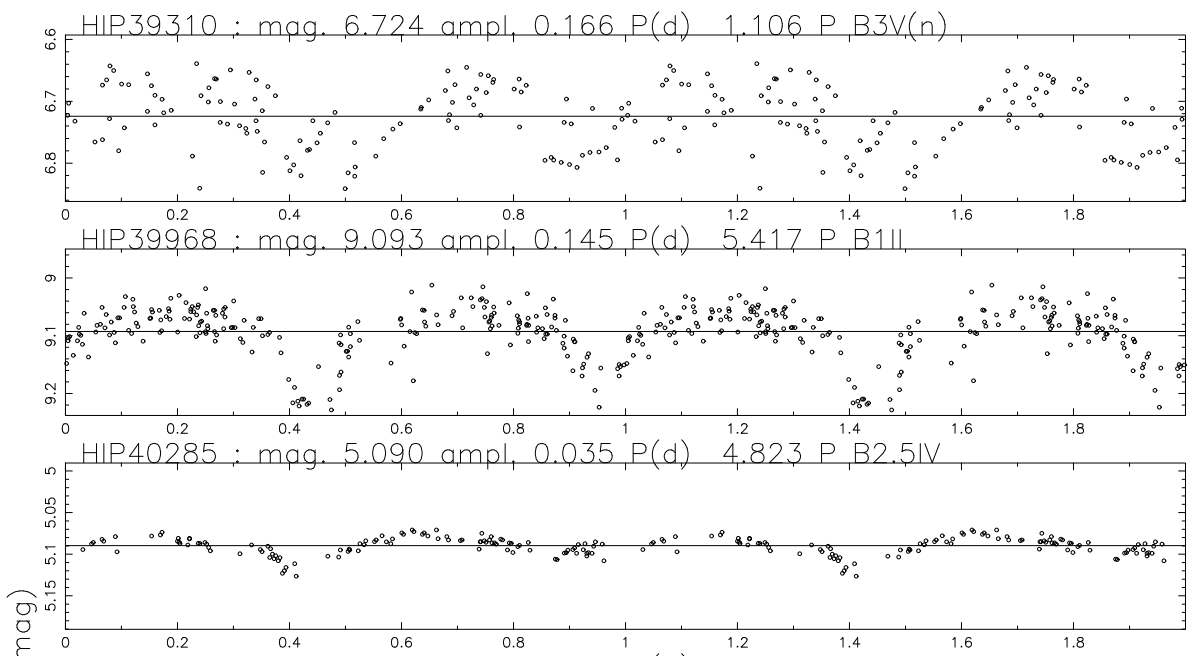

을
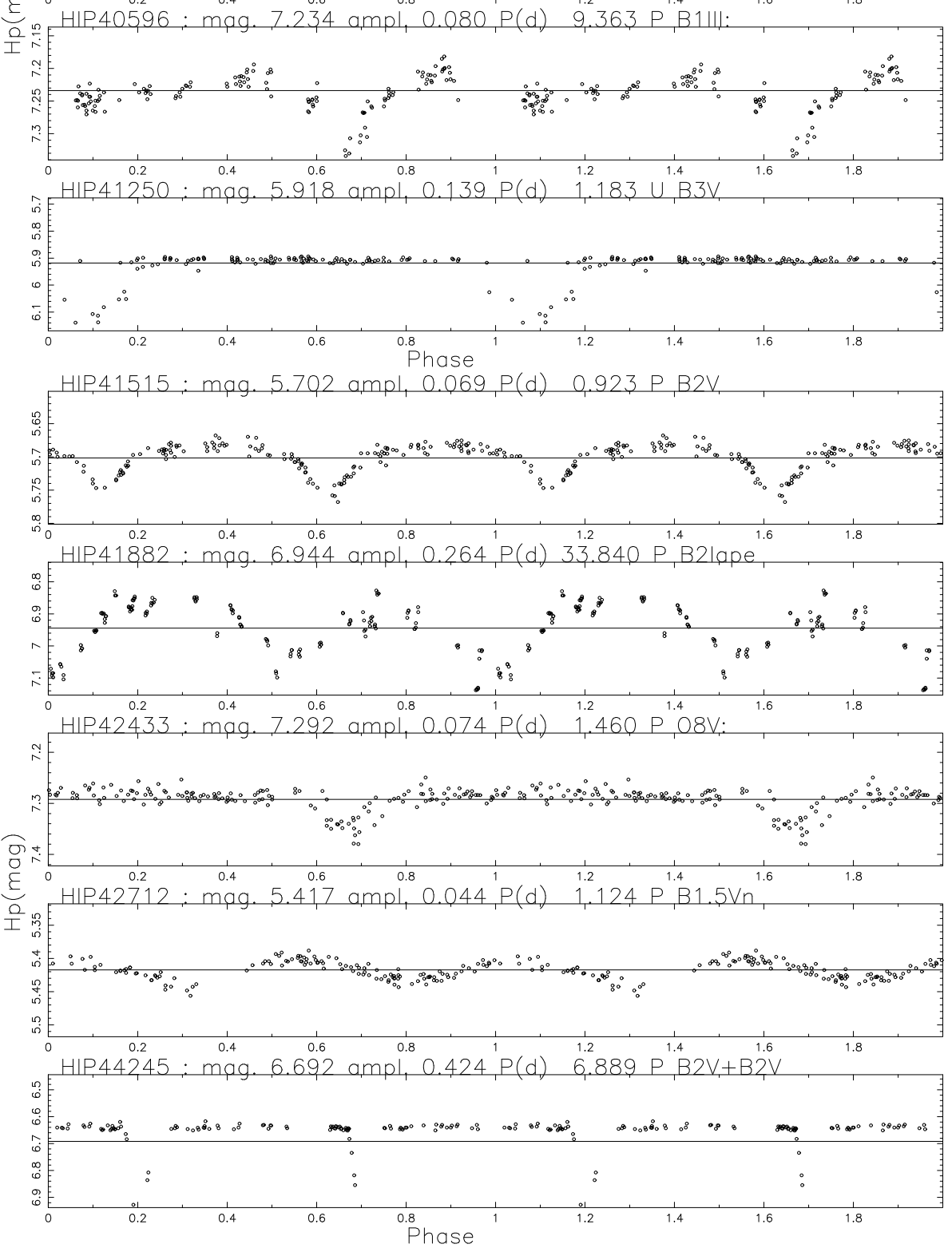

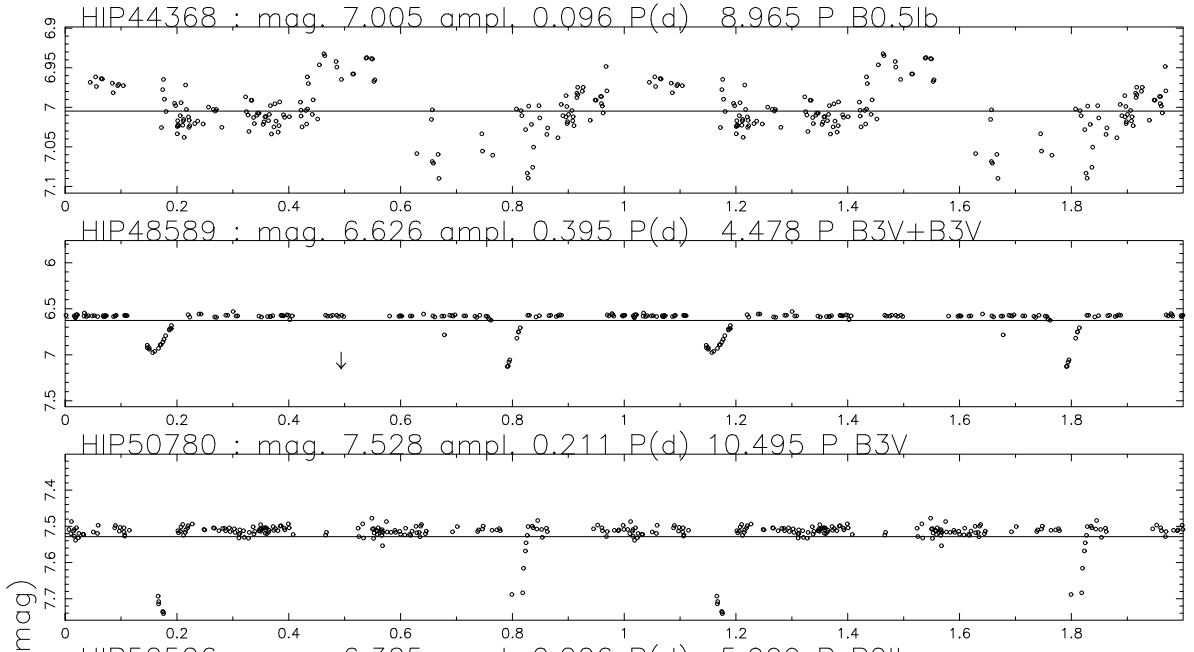

E HIP,52526: mag. 6.32,5 ampl, 0.206 P(d) 5,999 P, Bolb:
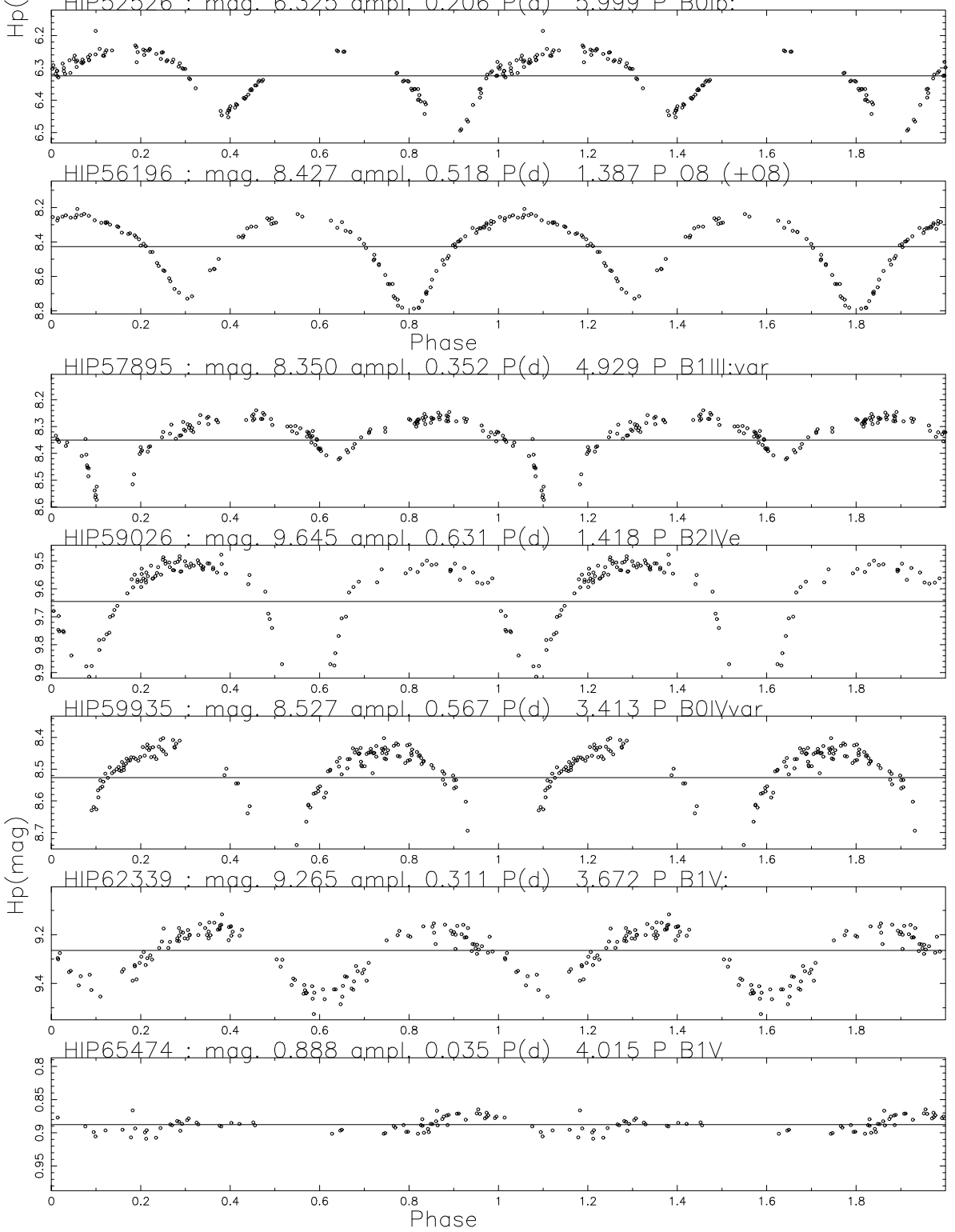

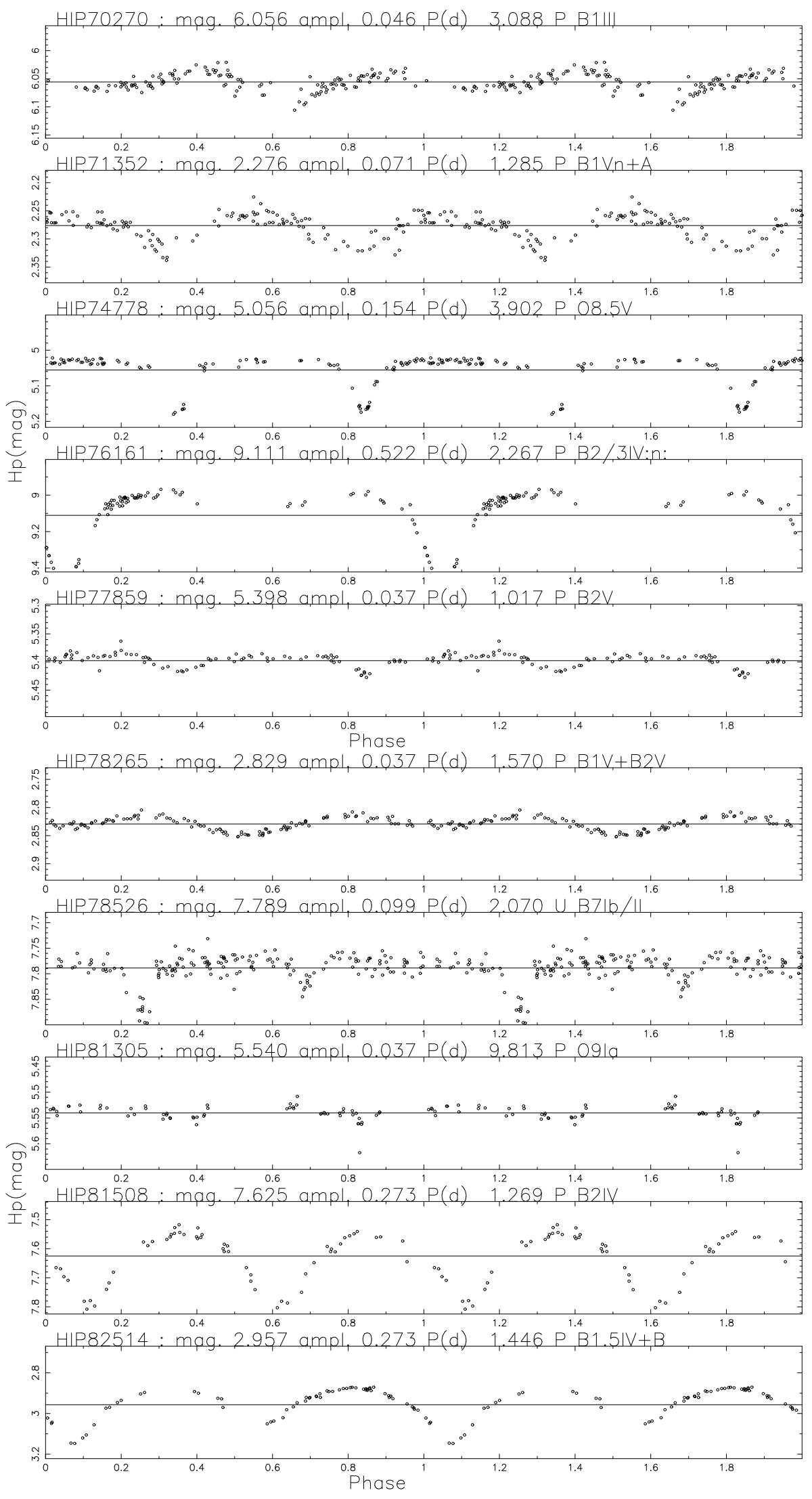

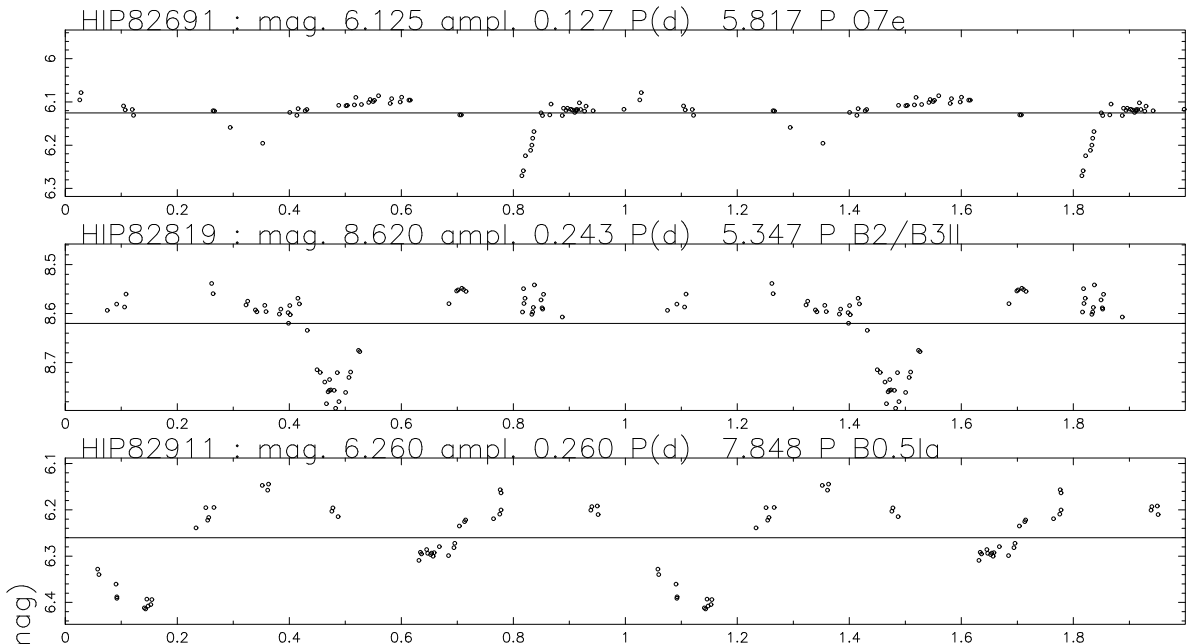

E HIP,83499: mag. $6.57,3 \mathrm{ampl}, 0.066, \mathrm{P}(\mathrm{d}) \quad 3,411 \mathrm{P}, 05 f$
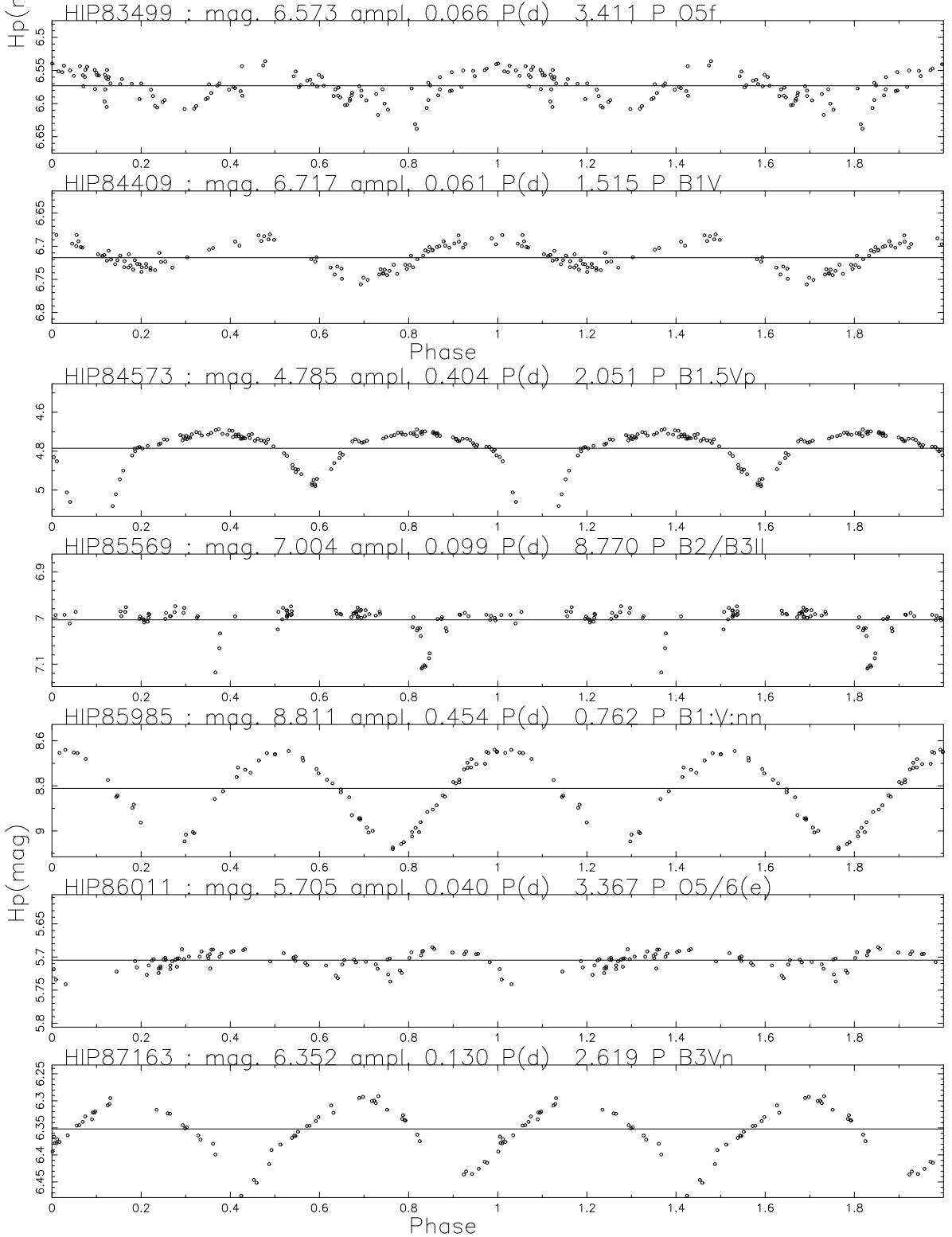

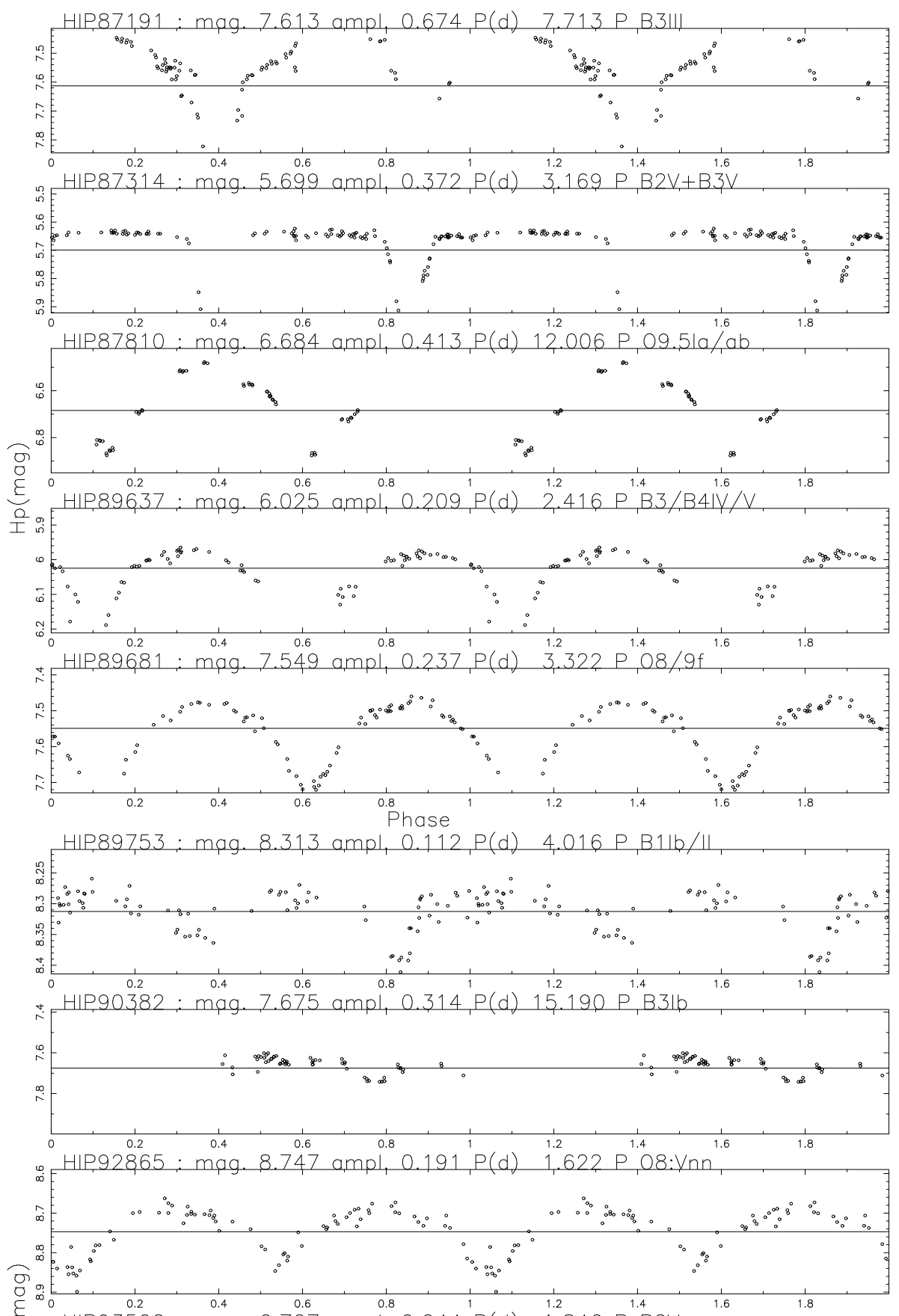

E HIP93502: mag. $6.7077^{0.2} \mathrm{ampl}_{1}^{0.8} 0.044^{1} \mathrm{P}(\mathrm{d}) \quad{ }_{1,846}^{1.2} \mathrm{P}_{1}^{1.4} \mathrm{~B} 2 \mathrm{~V}$
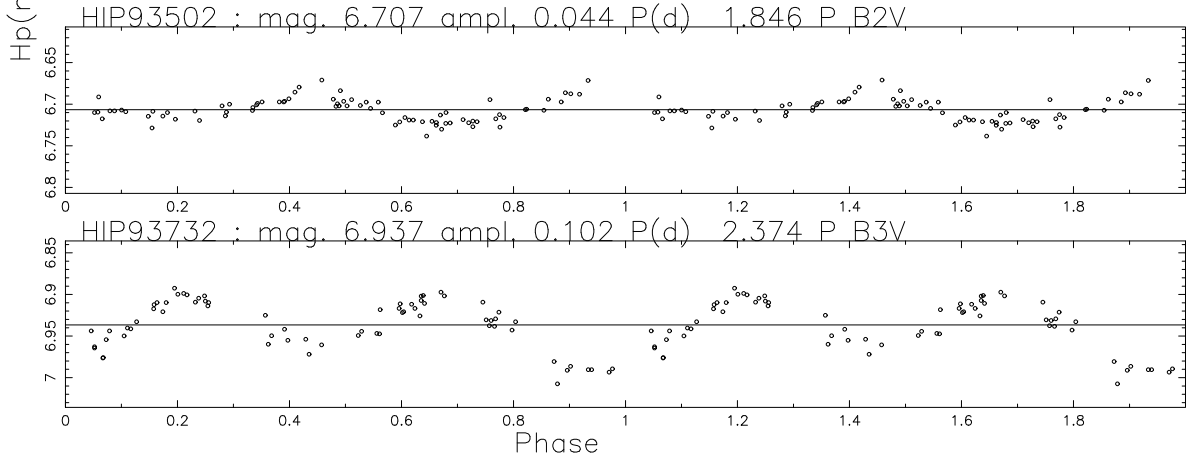

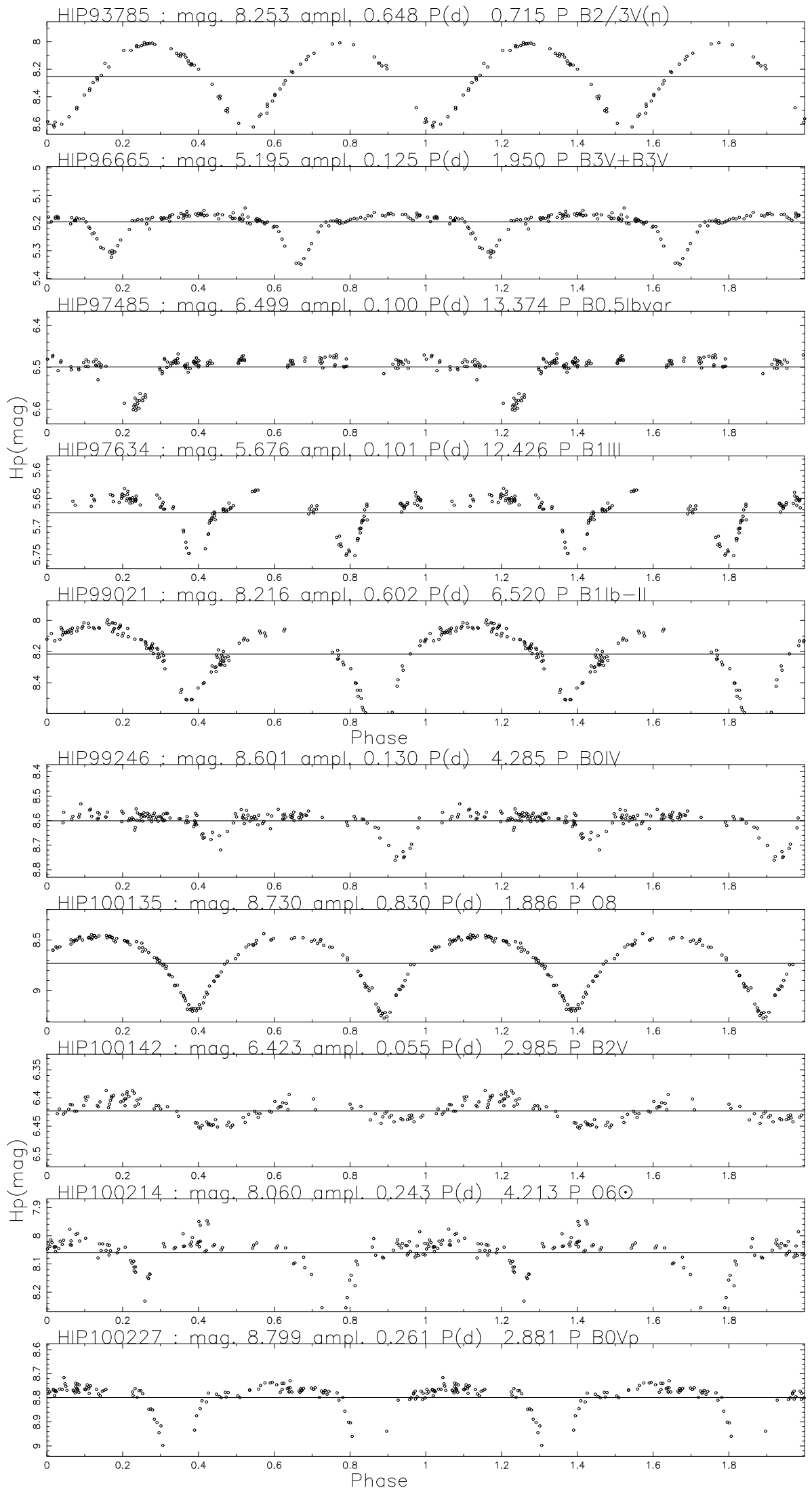


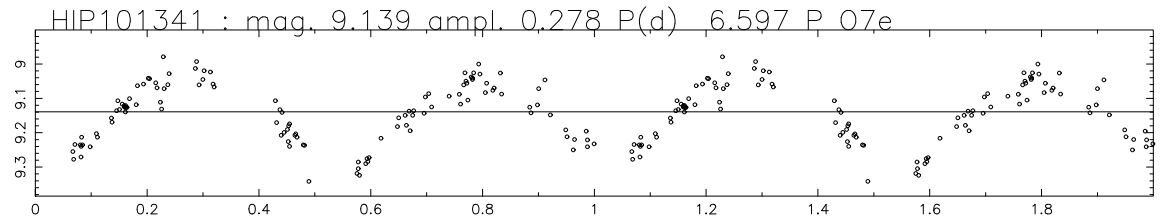

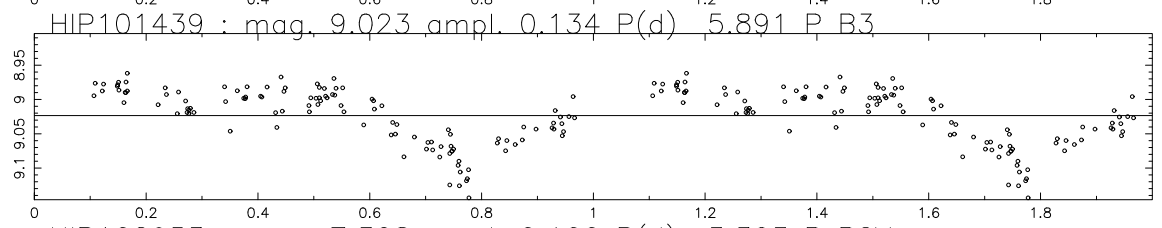
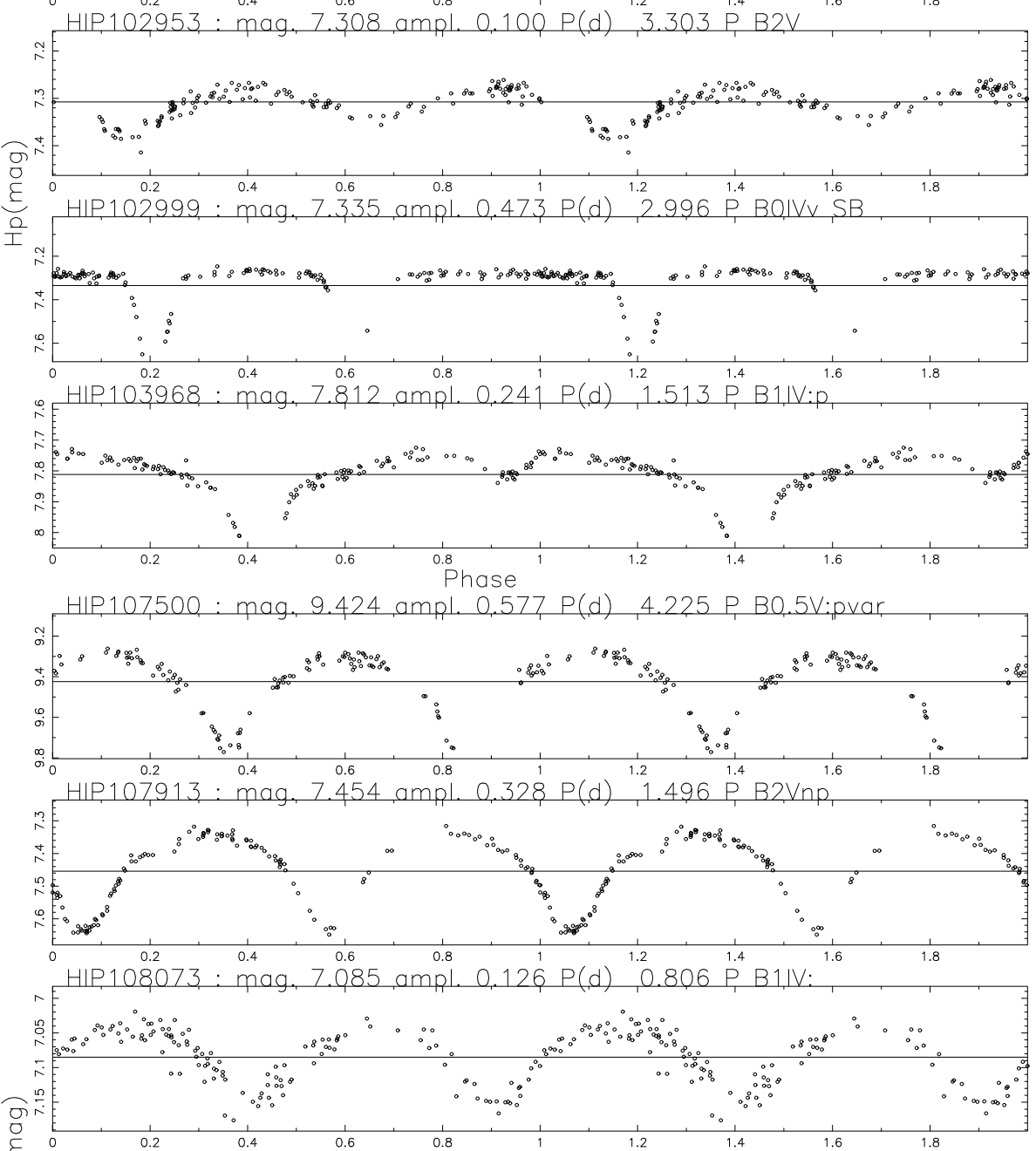

HIP, 108508: mag. $8.881 \mathrm{ampl} .0 .524 \mathrm{P}(\mathrm{d}), 1.677$ P B3V

으눙
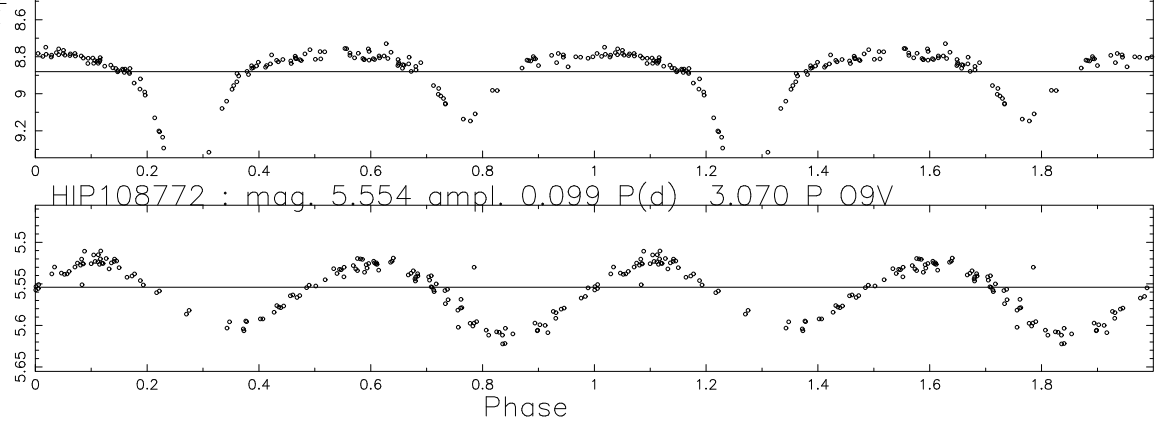

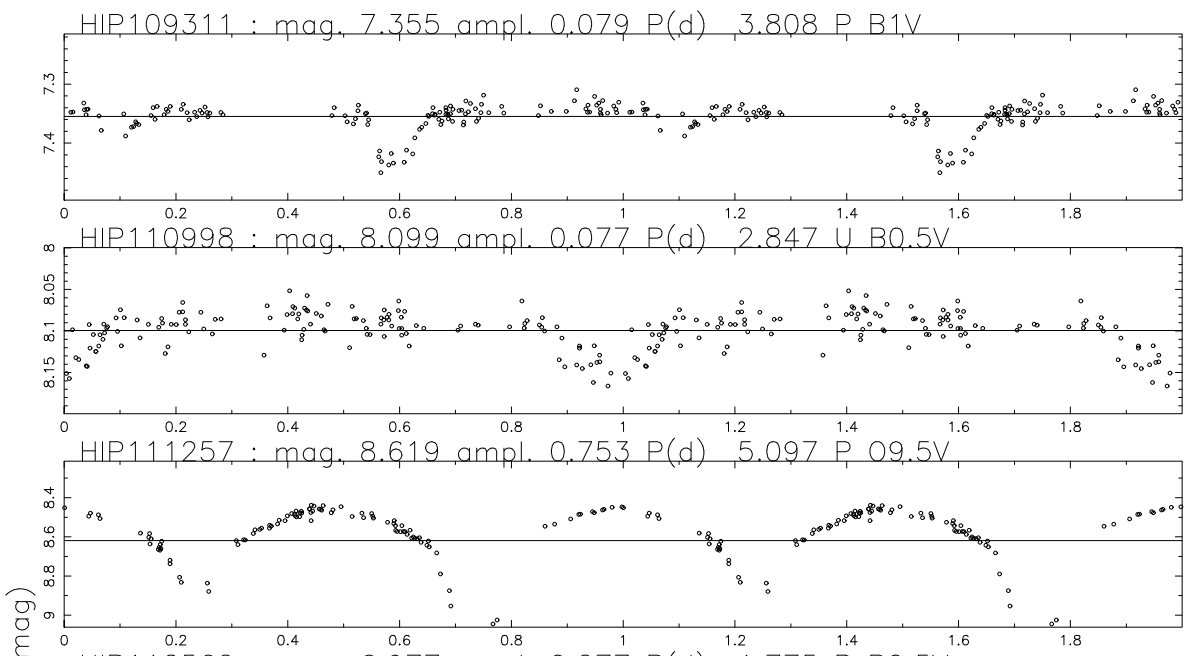

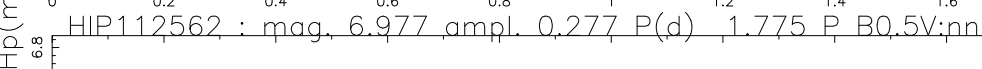
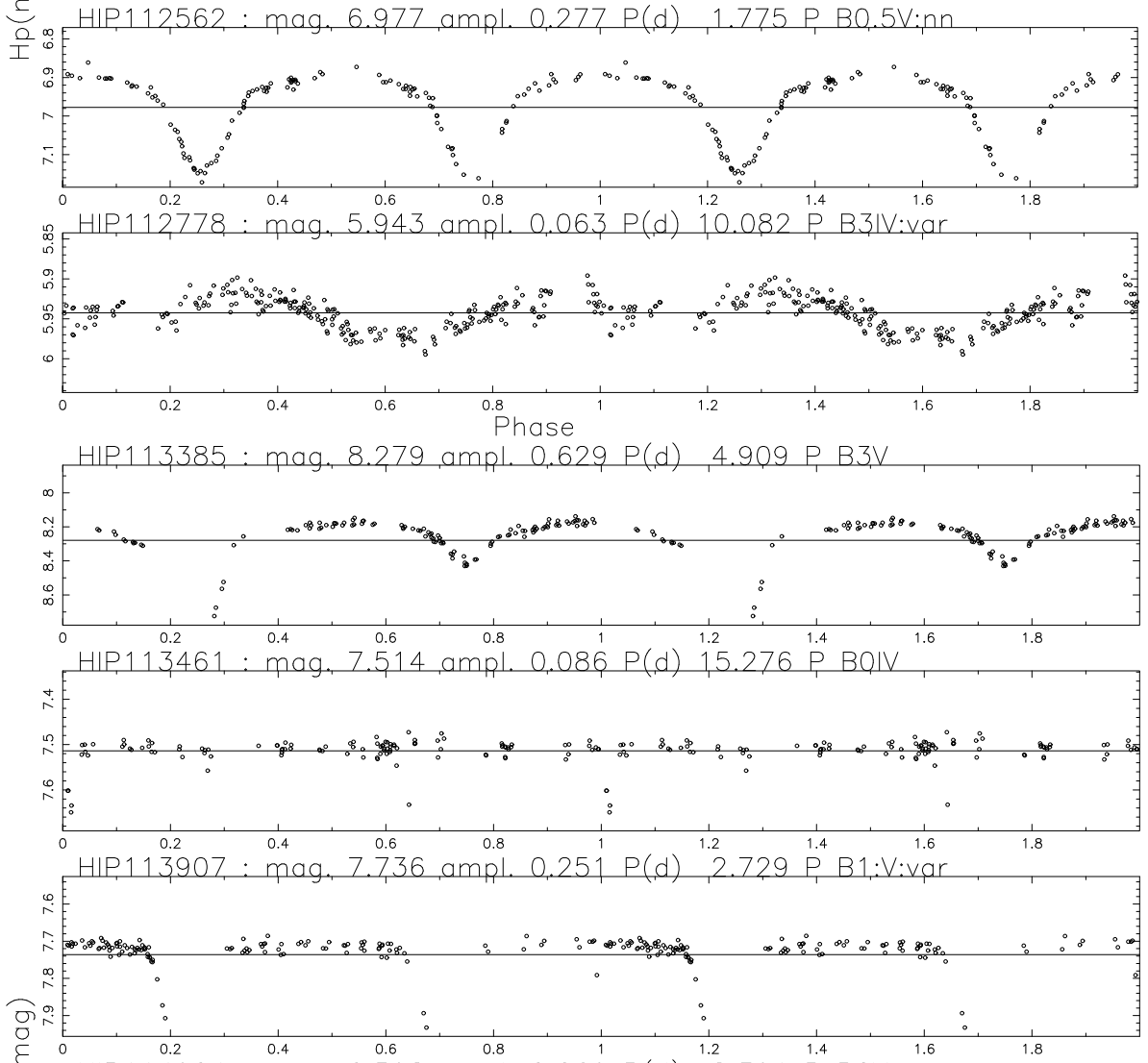

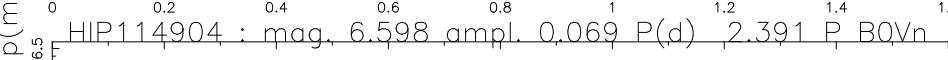
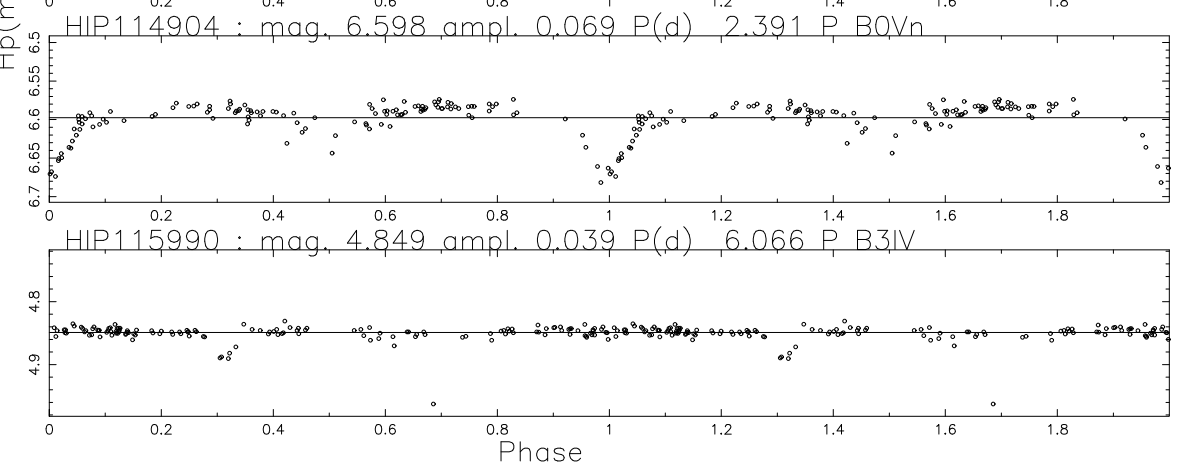
1178

L. Lefèvre et al.: Variability among OB-stars
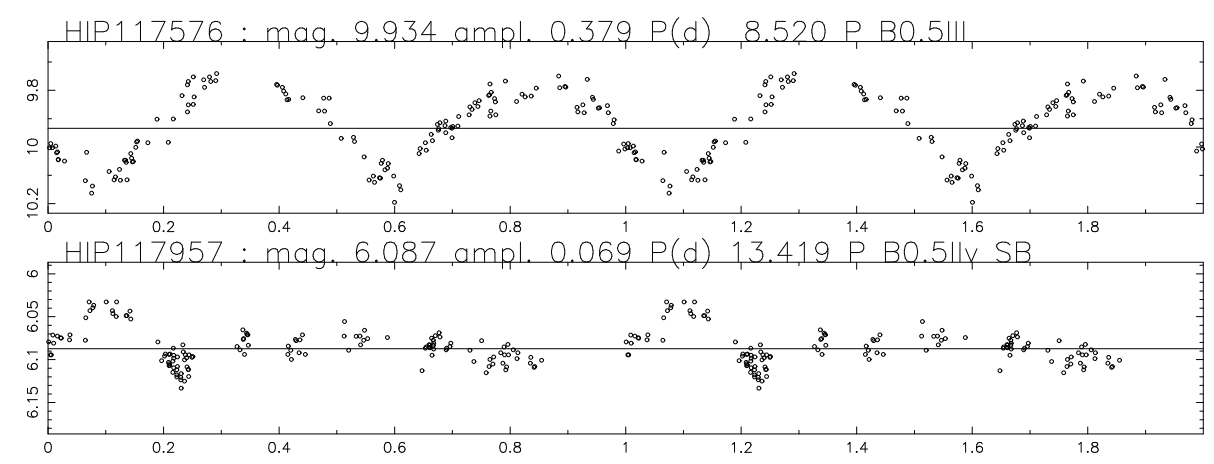


\section{B.2. Catalog of new periodic variable stars}
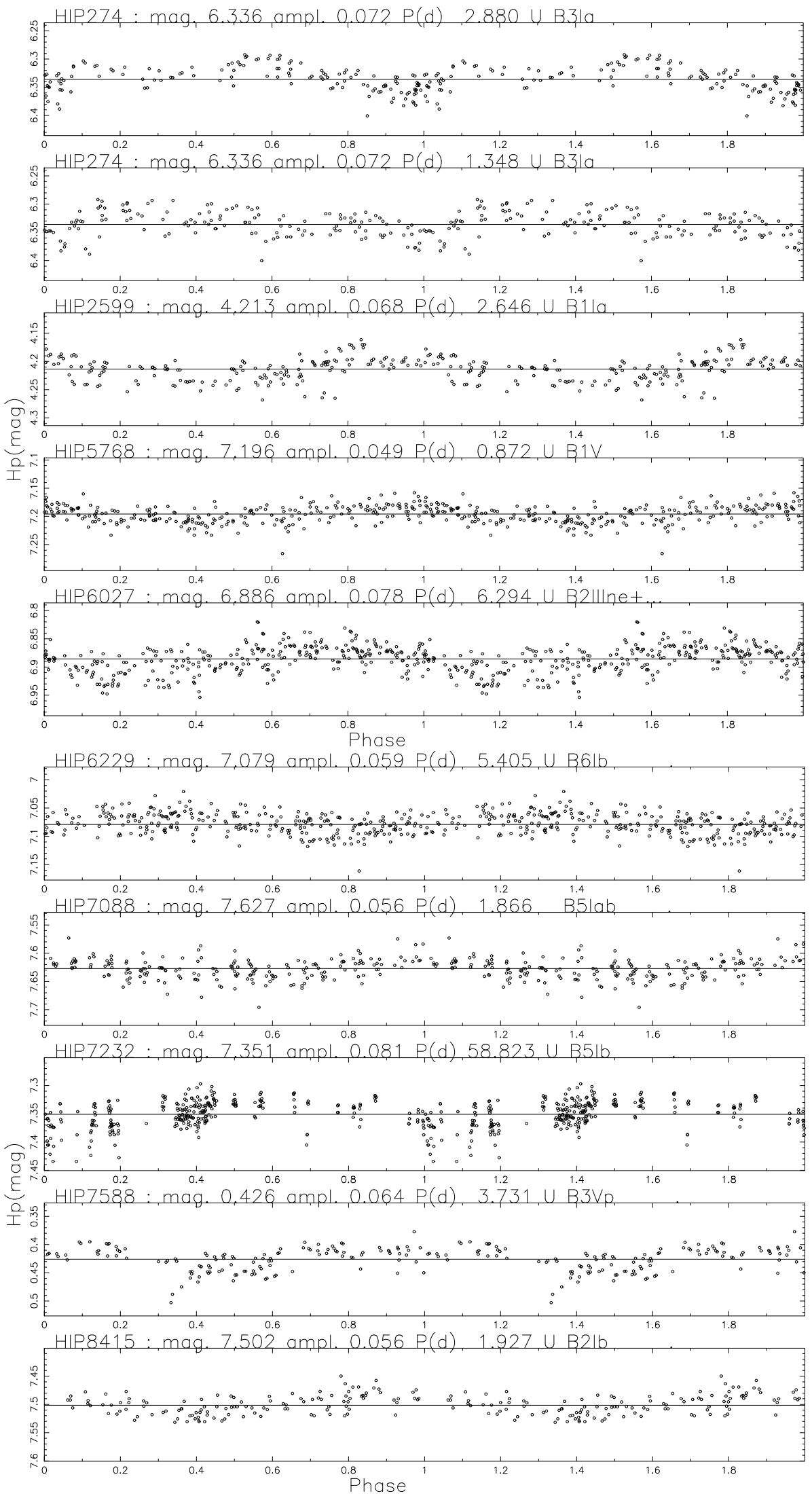

Fig. B.2. This figure plots $H p$ mag. vs. Phase. Epoch is arbitrarily JD-2 $440000=7800$, which is the beginning of the HIPPARCOS observations. The $H p$ magnitude, mean amplitude and periods are accurate to $\sim 0.1 \%$. 


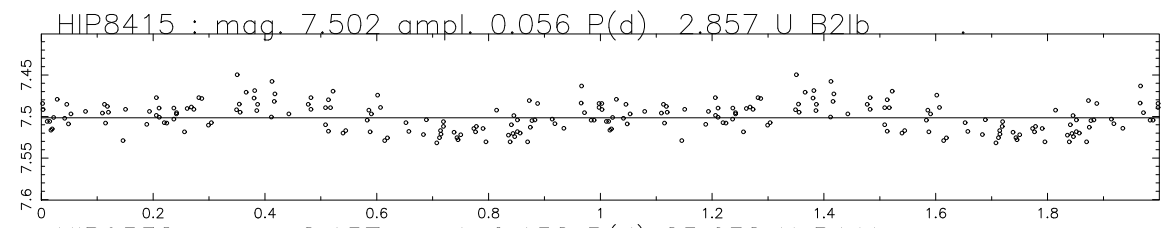

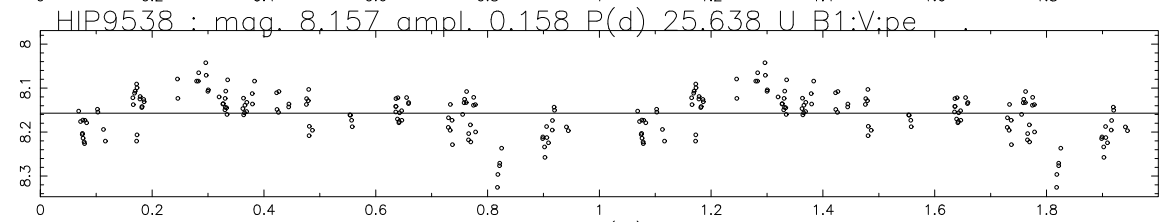

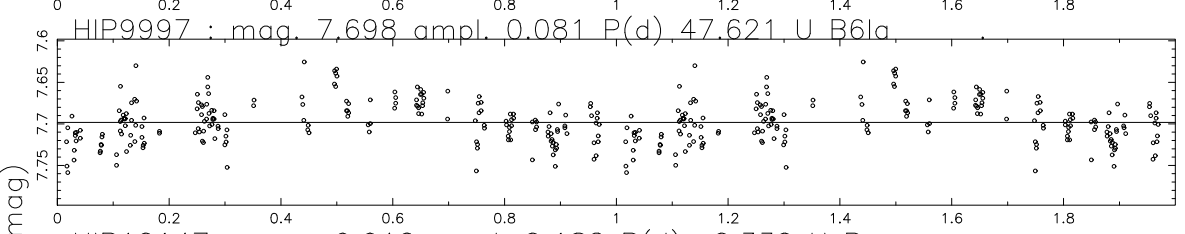

$\stackrel{2}{1}$

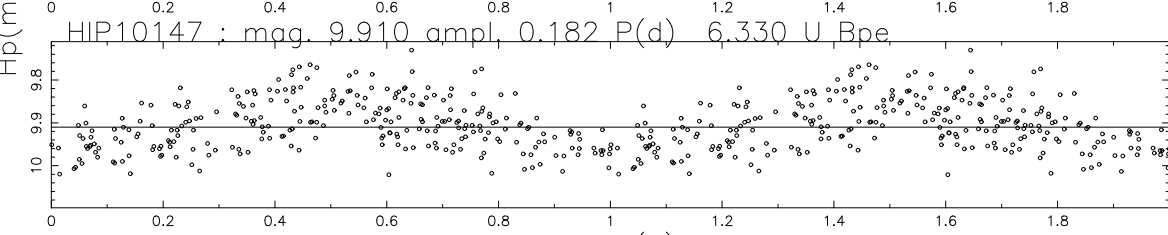

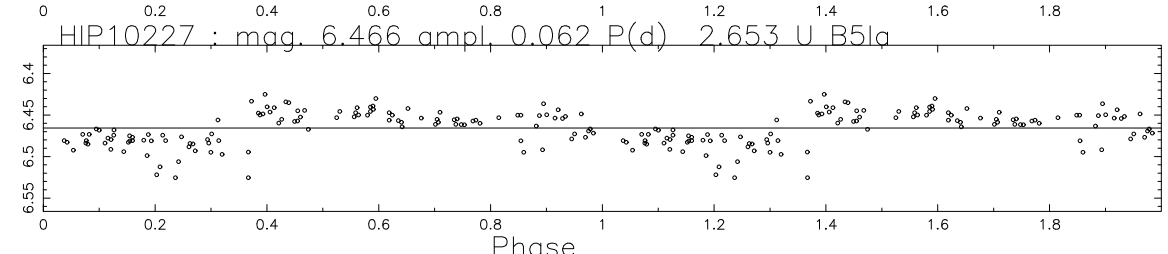

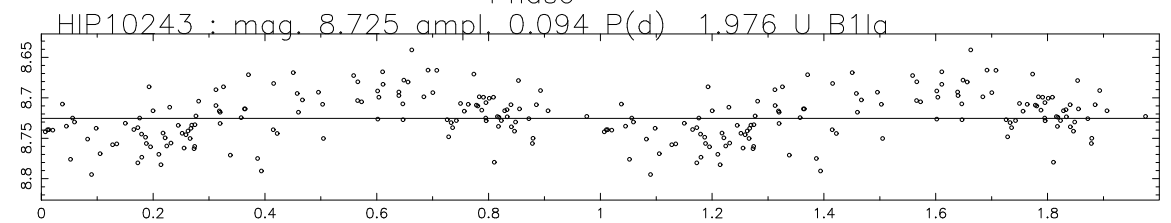

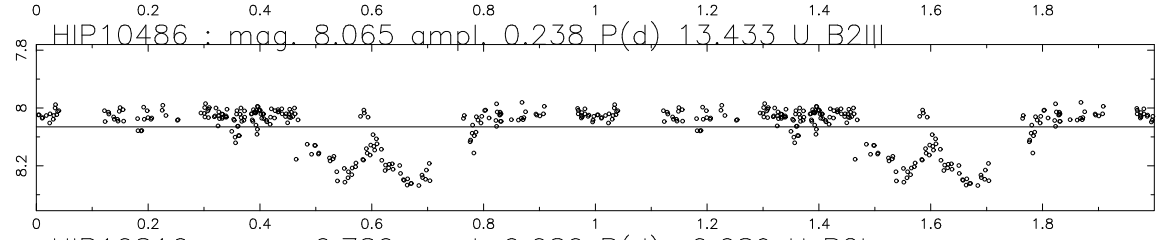

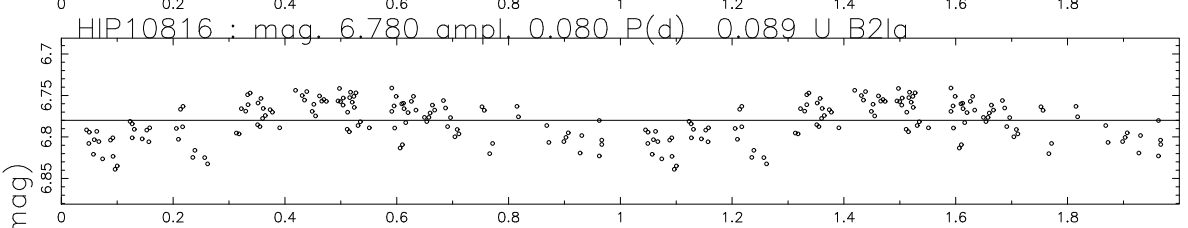

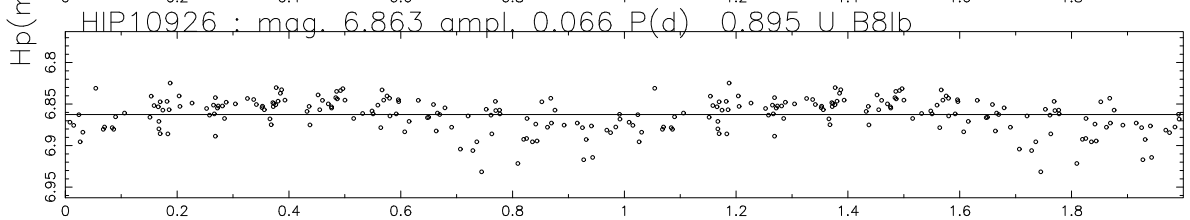

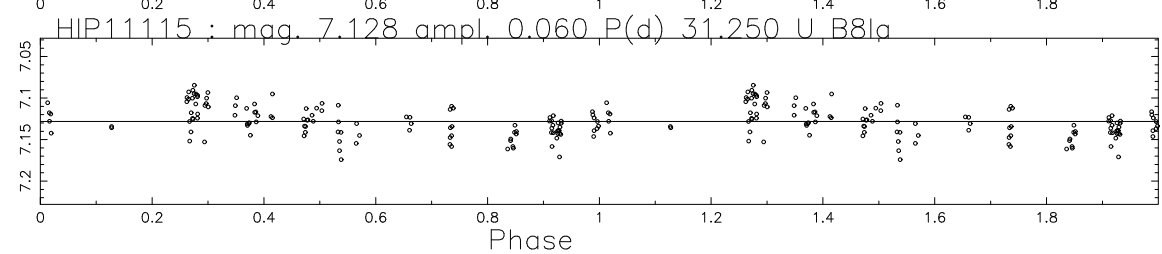




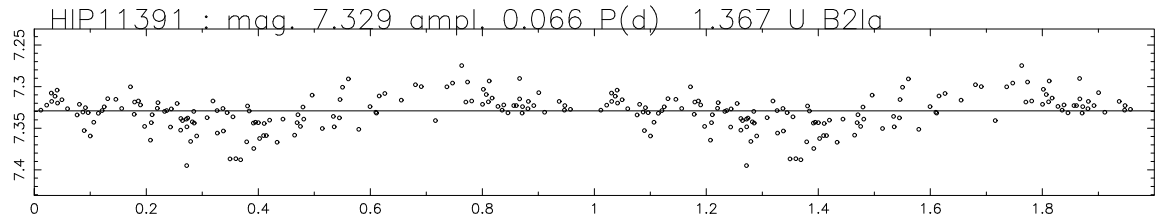

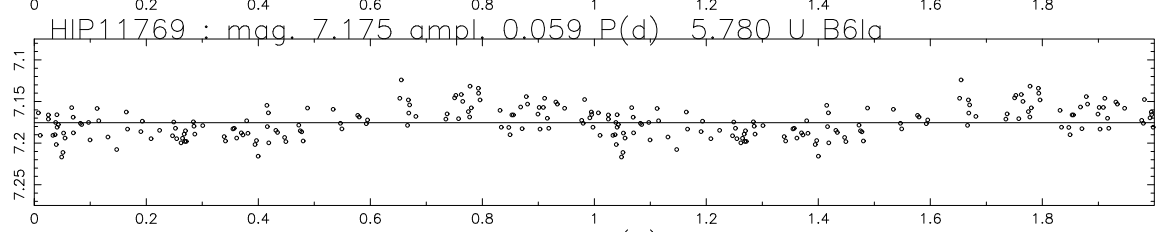

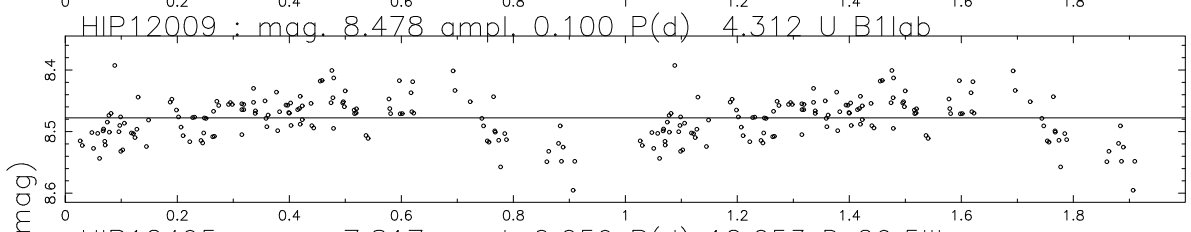

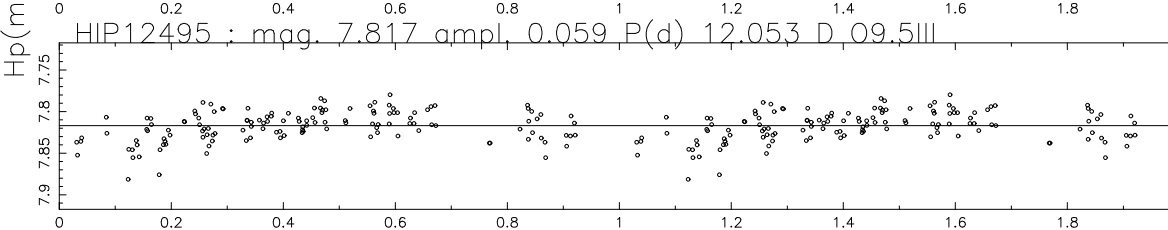
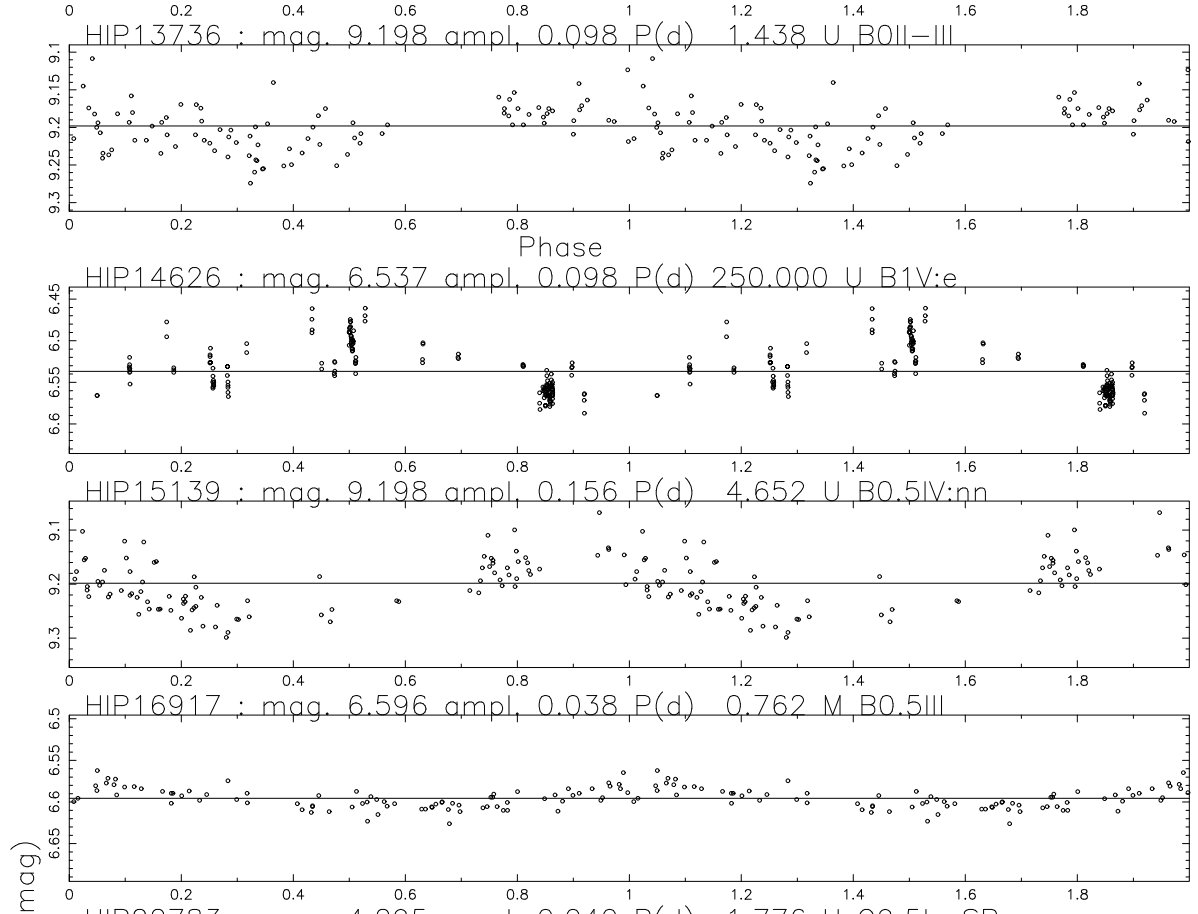

o f HIP,227,83: mag. $4.295 \mathrm{ampl}, 0.040, P(d) \quad 1,776$ U, 09.51a SB

오
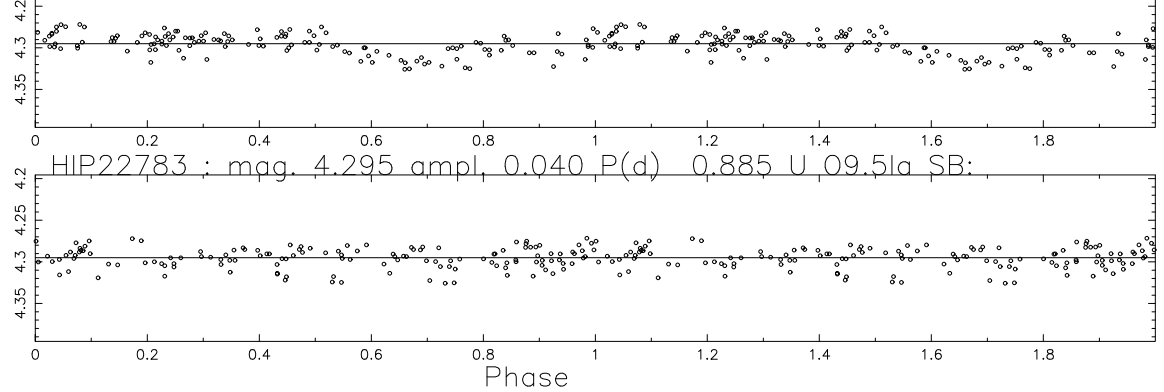

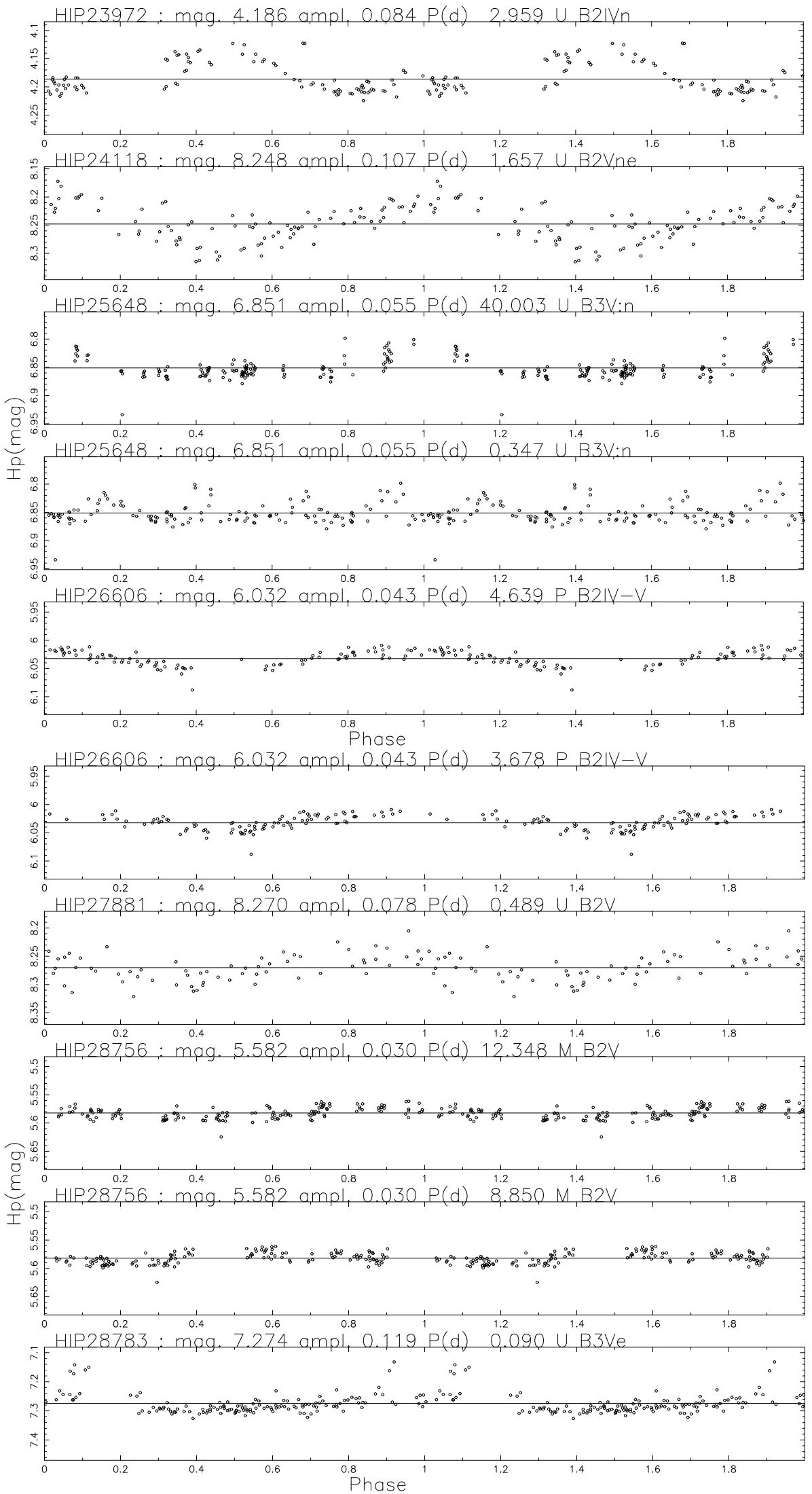

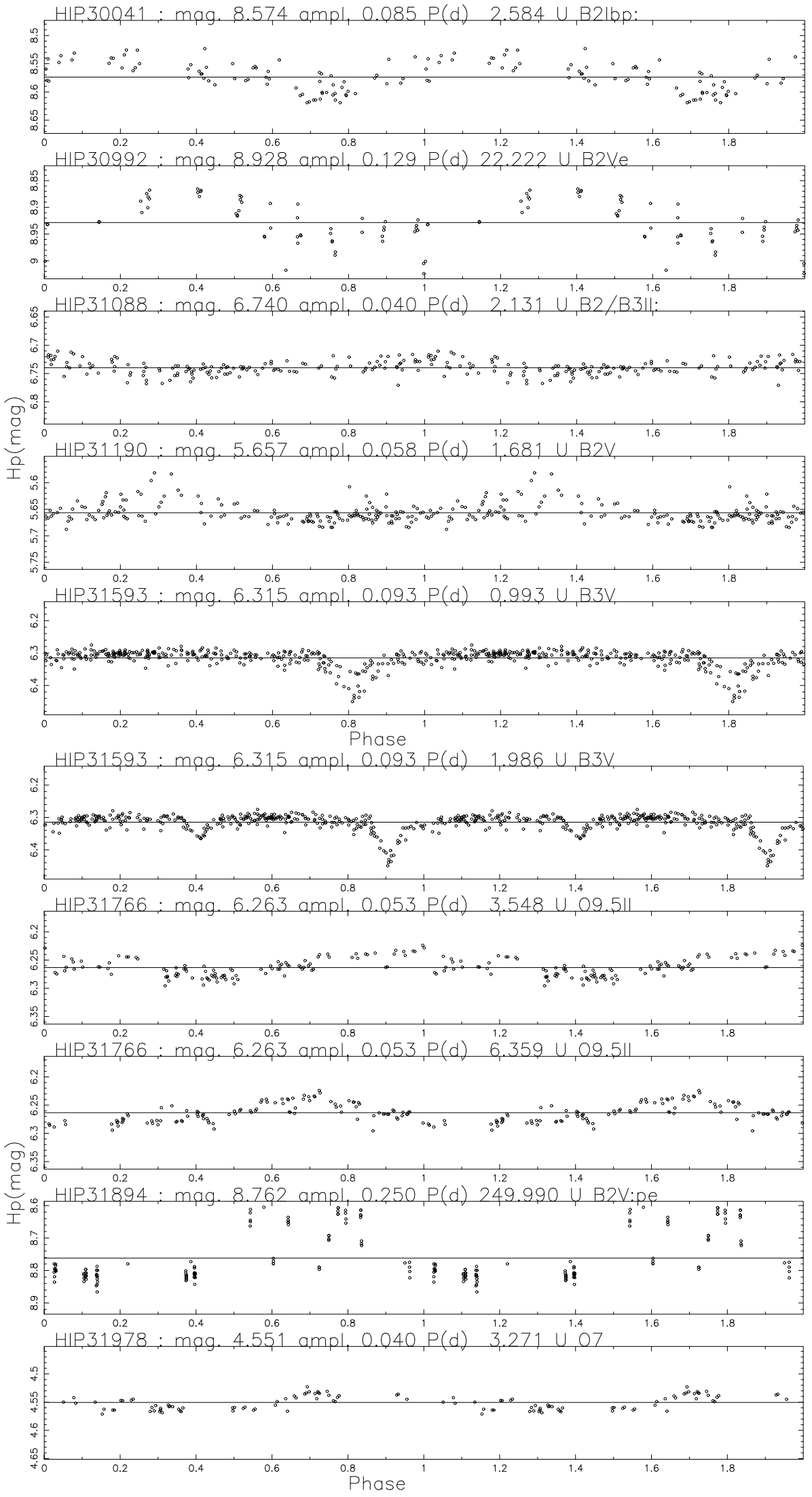

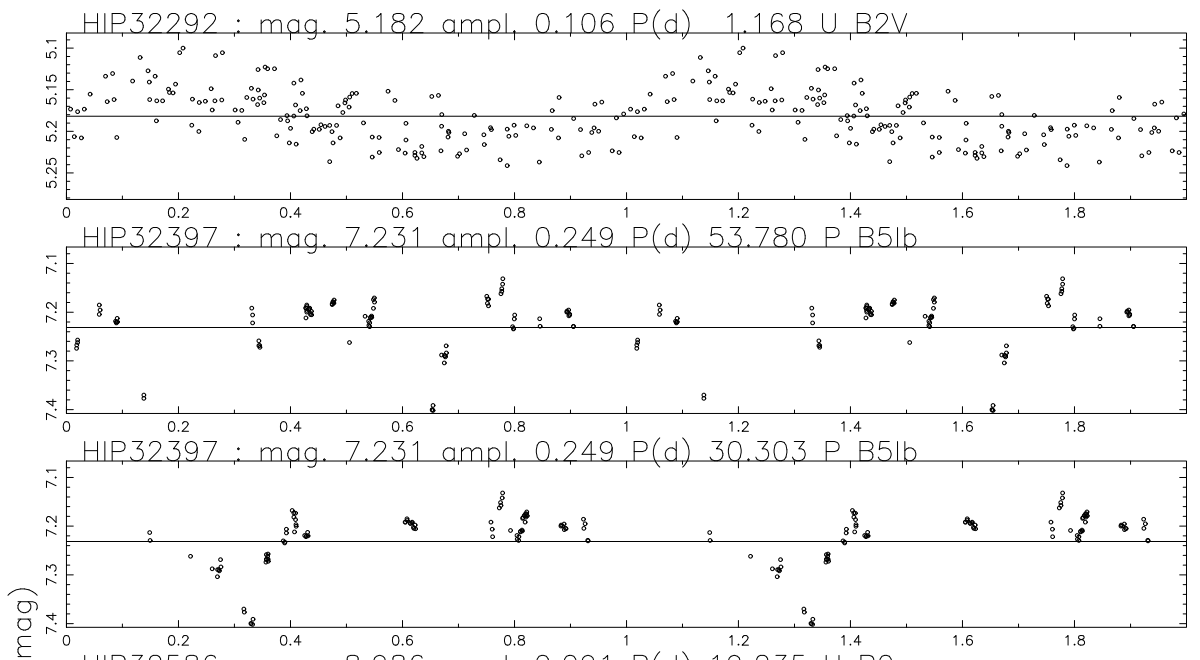

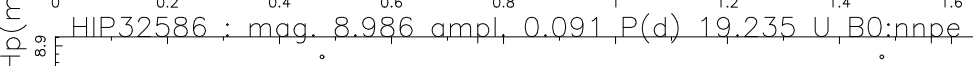
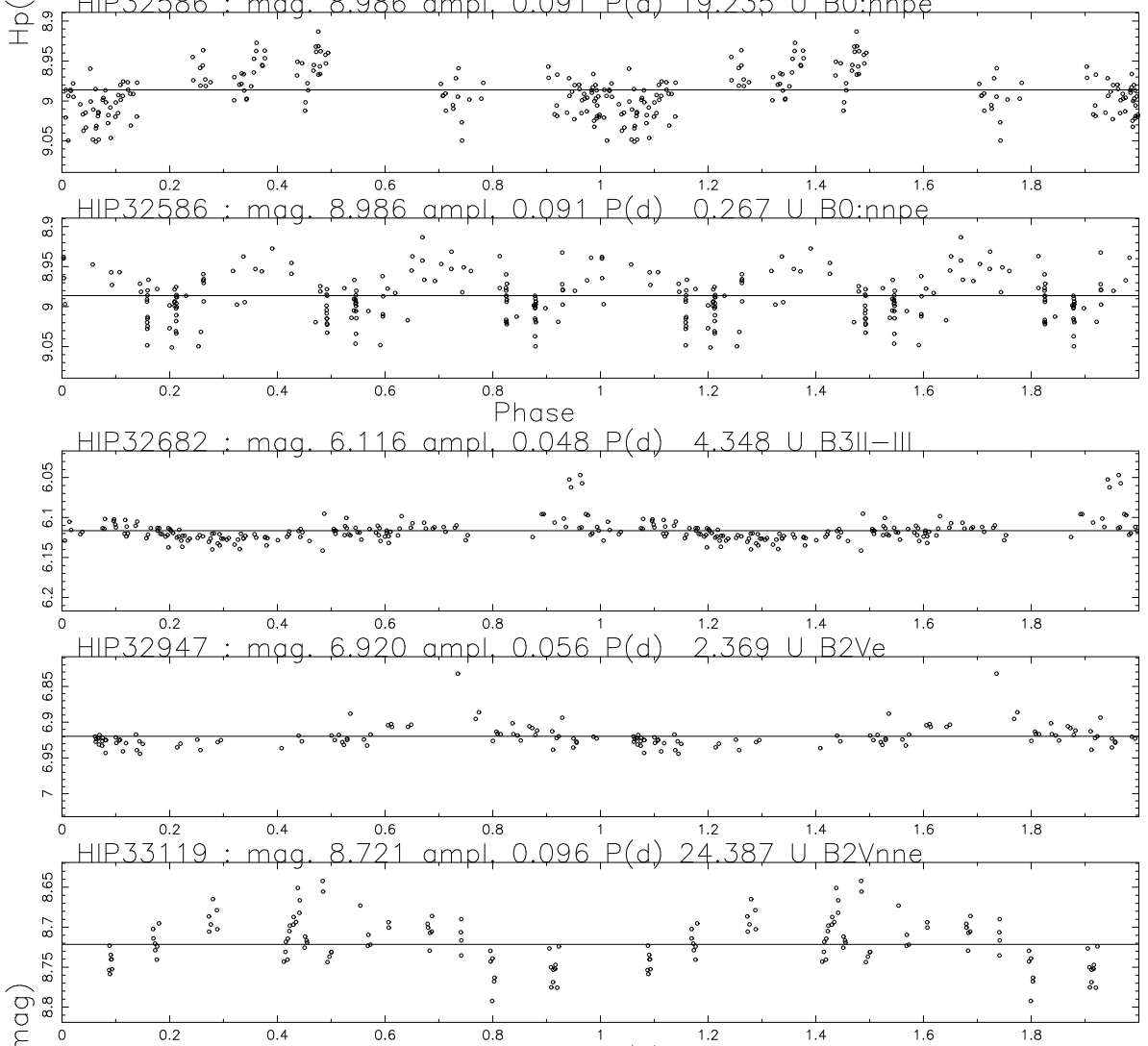

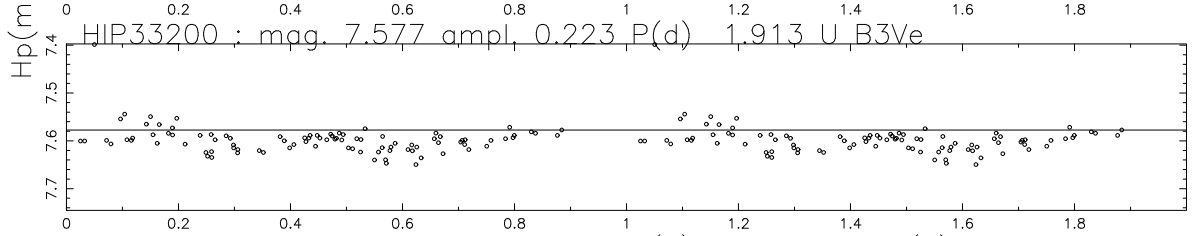

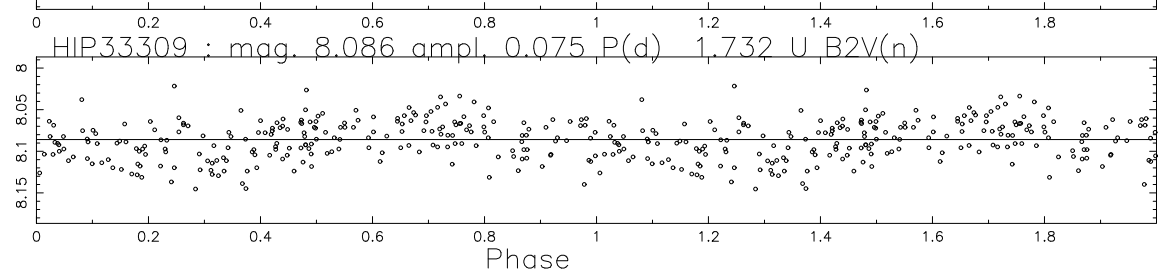



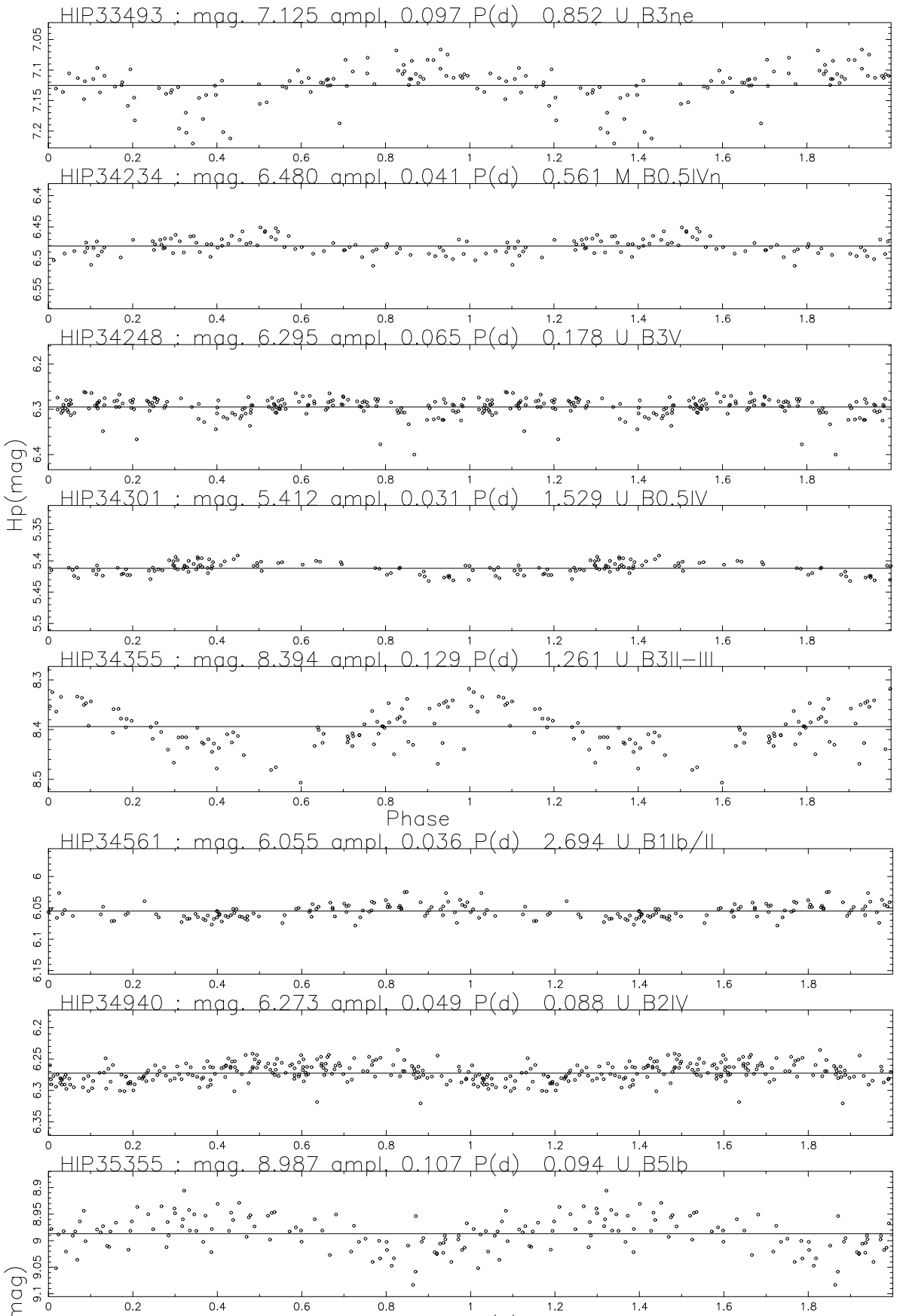

E HIP,35355: mag. $8.987 \mathrm{ampl}, 0.107, \mathrm{P}(\mathrm{d}) 2,698$ U $\mathrm{B} 5 \mathrm{lb}$
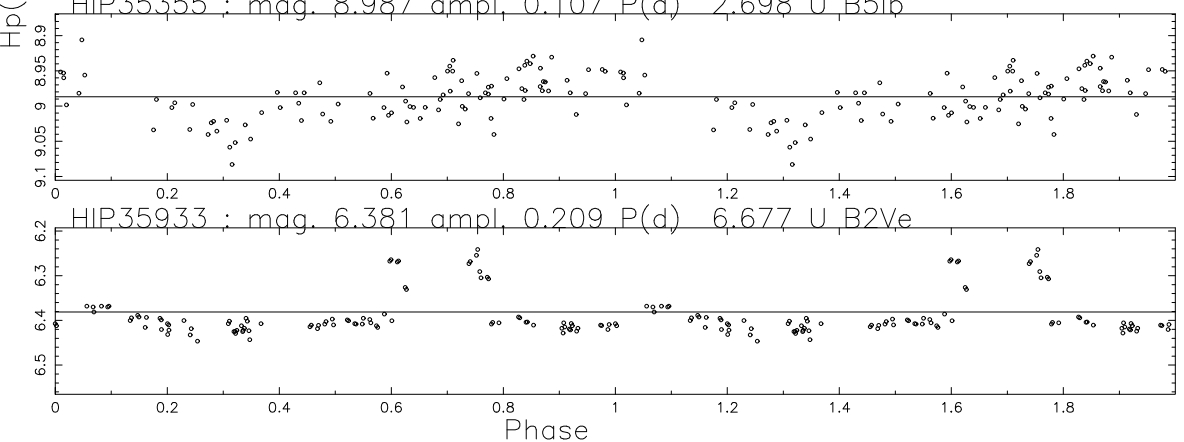

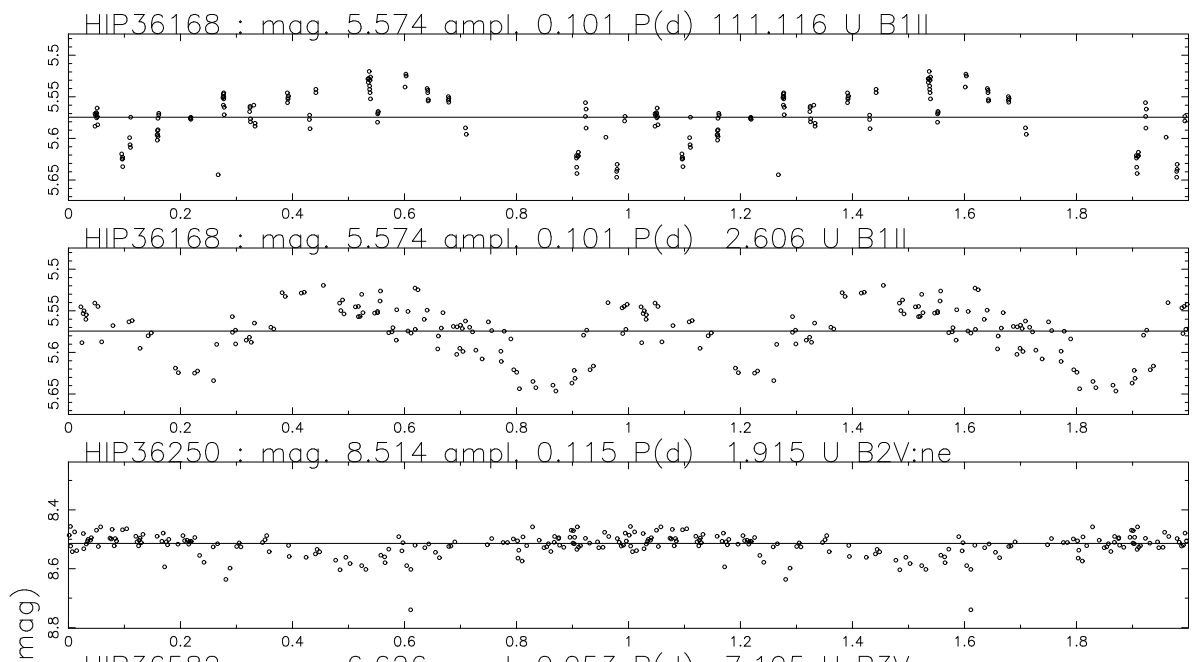

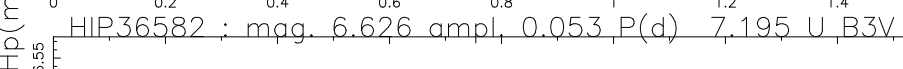

1
0
0

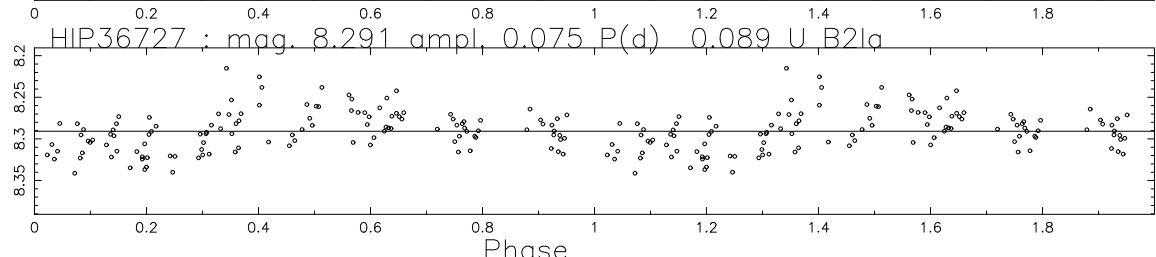

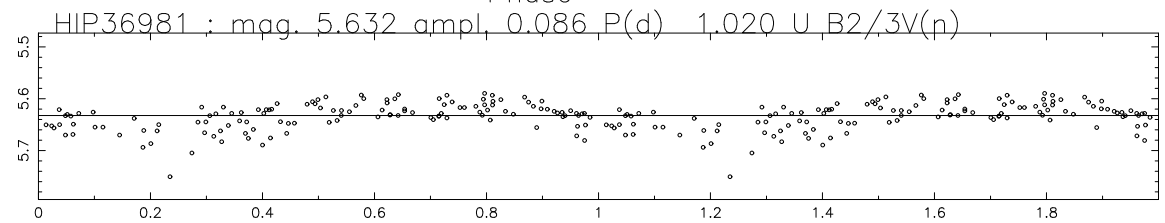

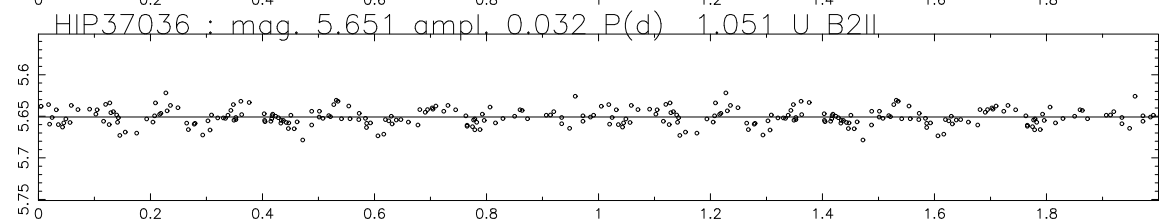

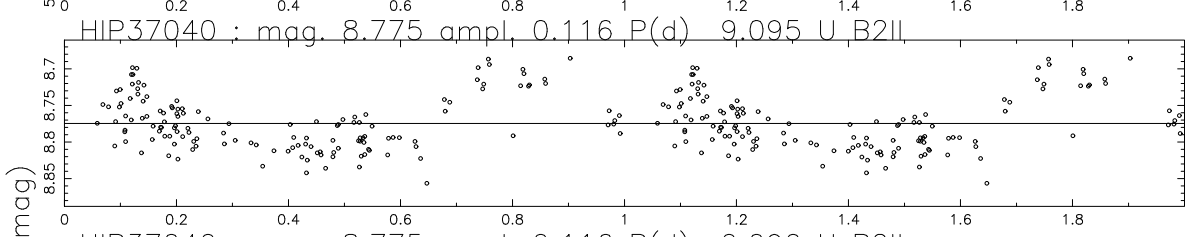
HIP,37040: mag. $8.77,5 \mathrm{ampl}, 0.116, \mathrm{P}(\mathrm{d}) \quad 0,090 \cup \mathrm{U}, \mathrm{B} 2$

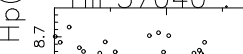

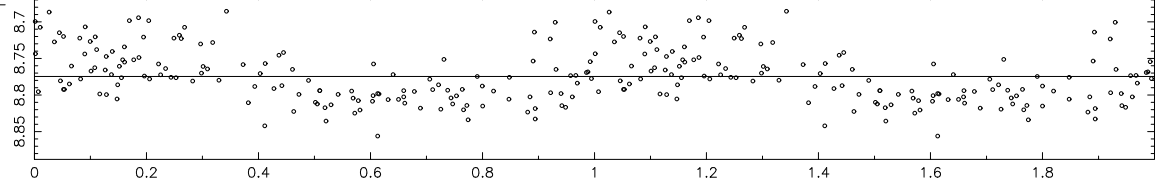

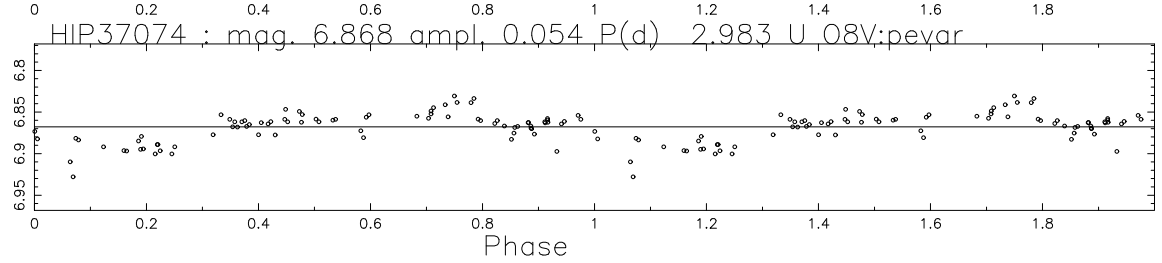



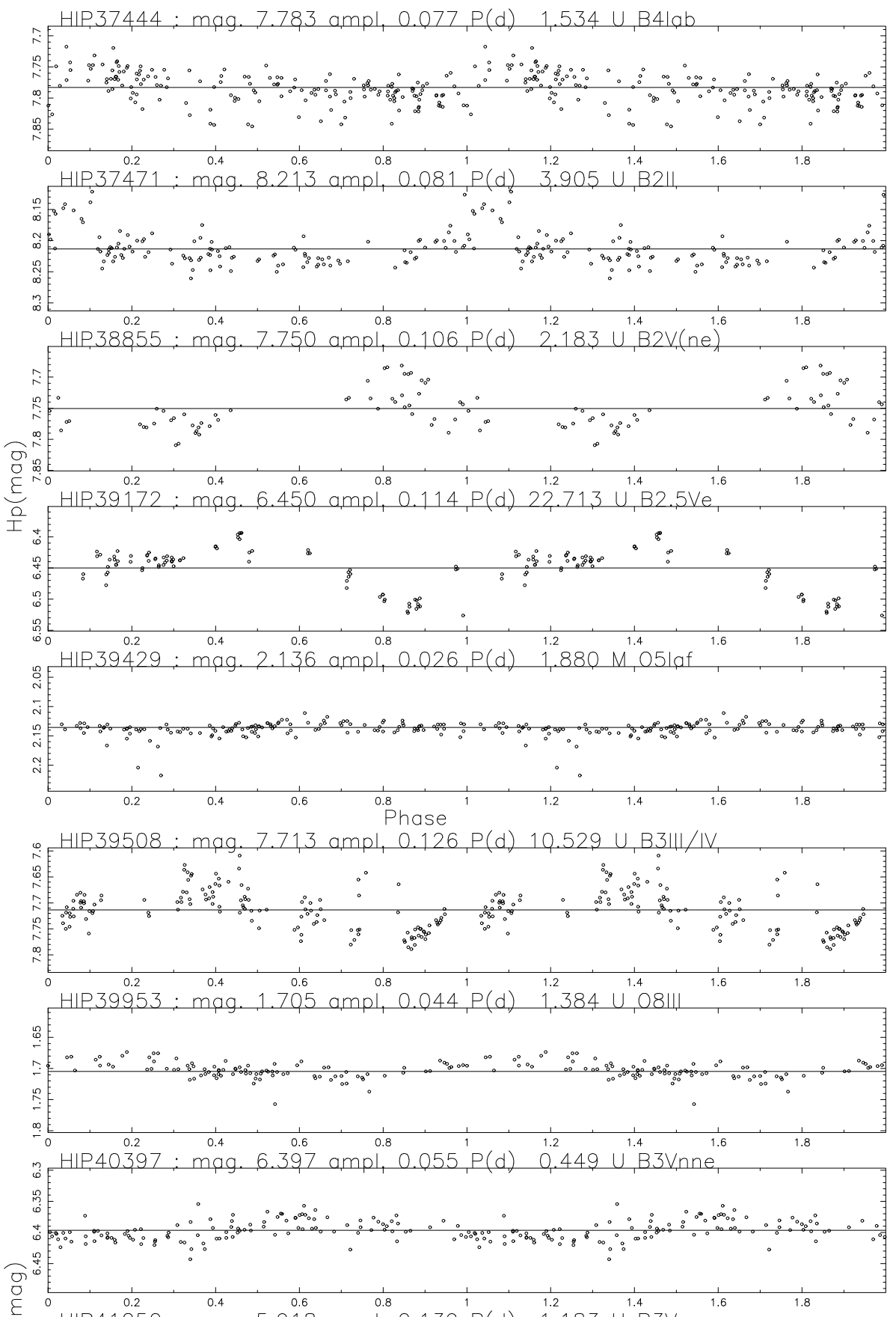

हf HIP,412,50: mpg. $5.91,8 \mathrm{ampl}, 0.139, \mathrm{P}(\mathrm{d}) \quad 1,183 \mathrm{U}_{1}^{1.4} \mathrm{~B} 3 \mathrm{~V}$,
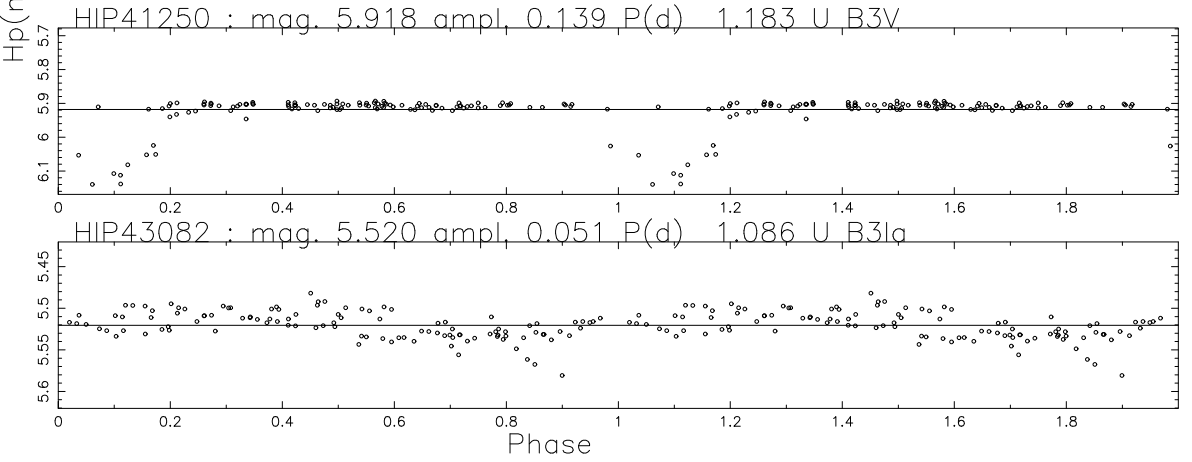

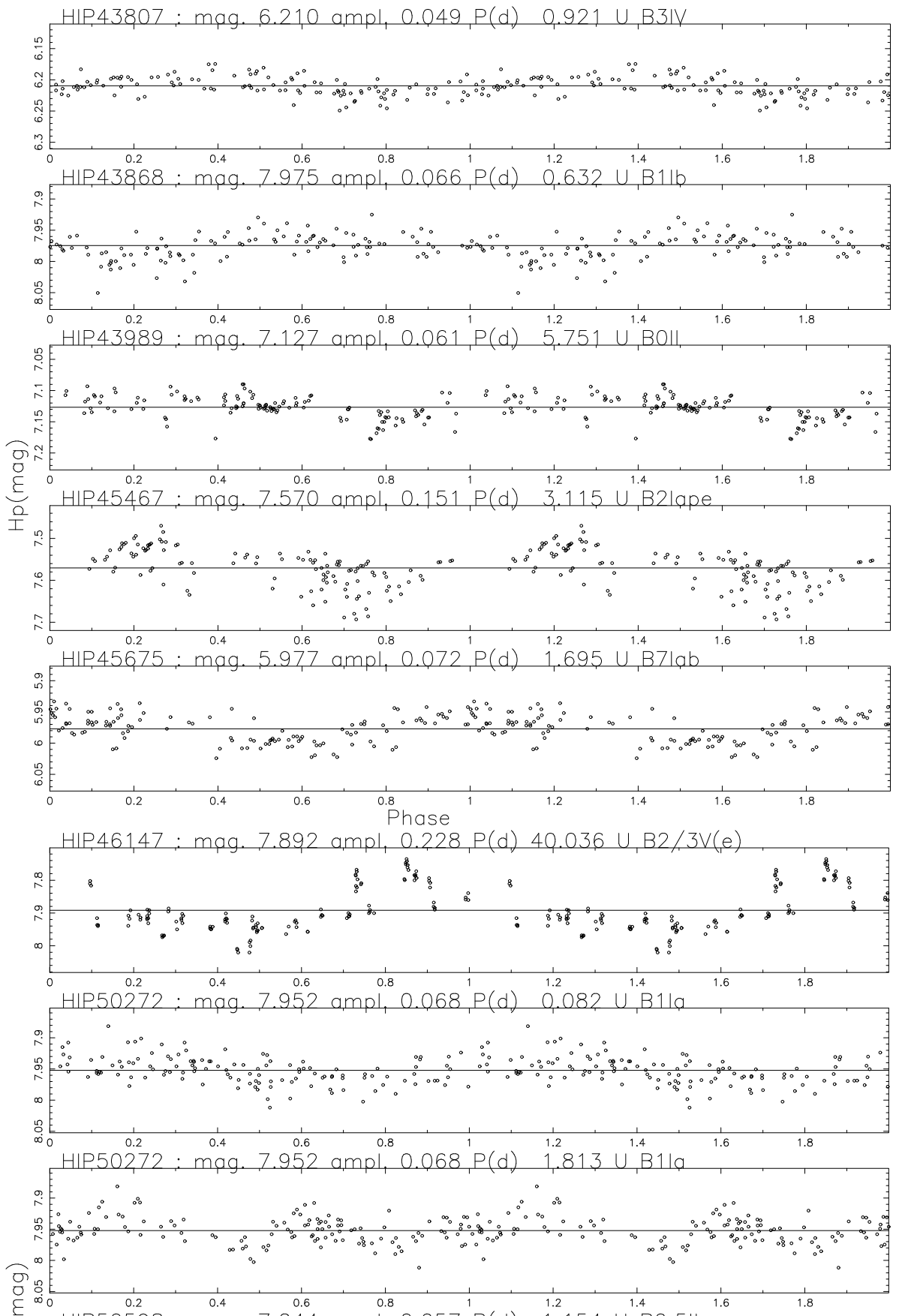

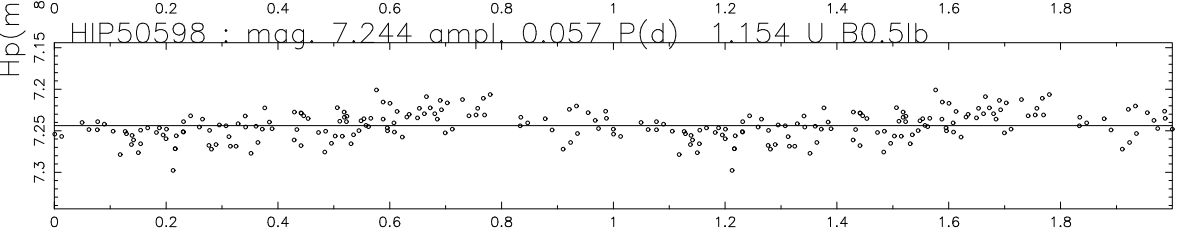

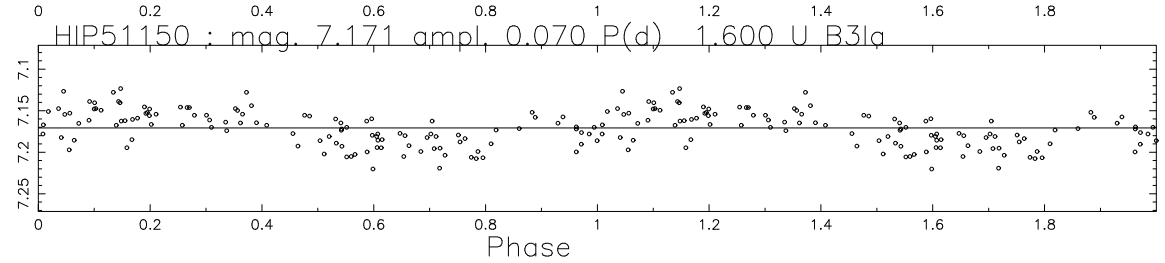



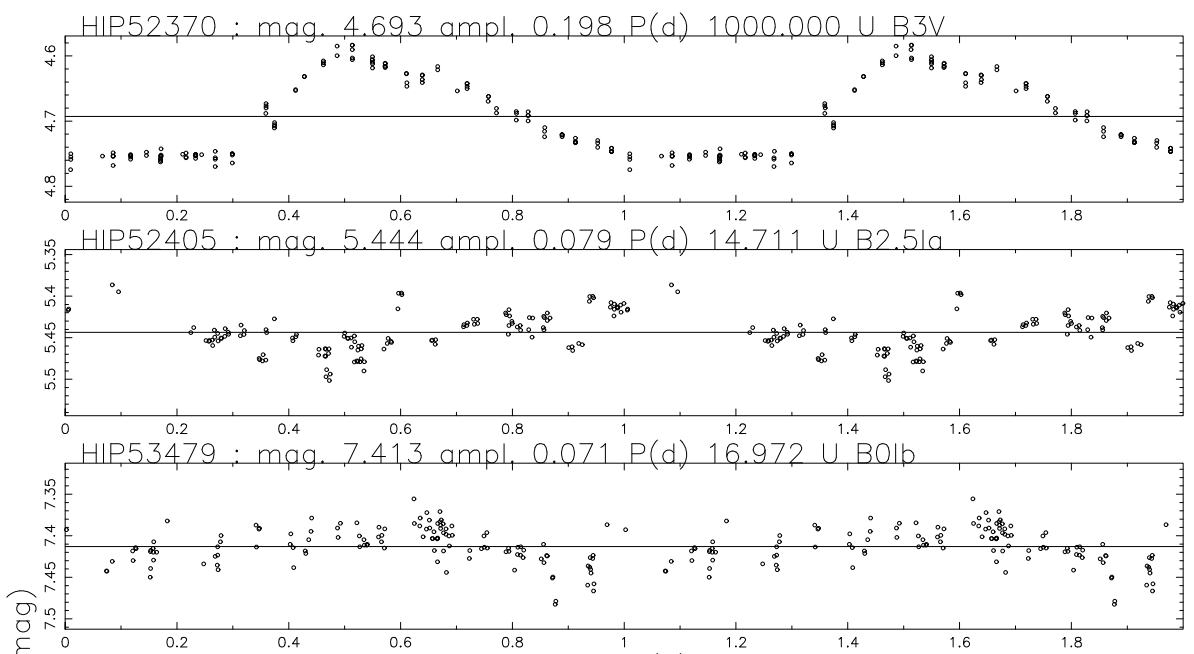

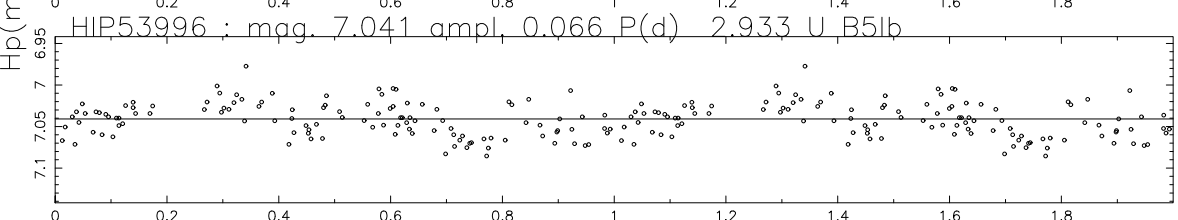
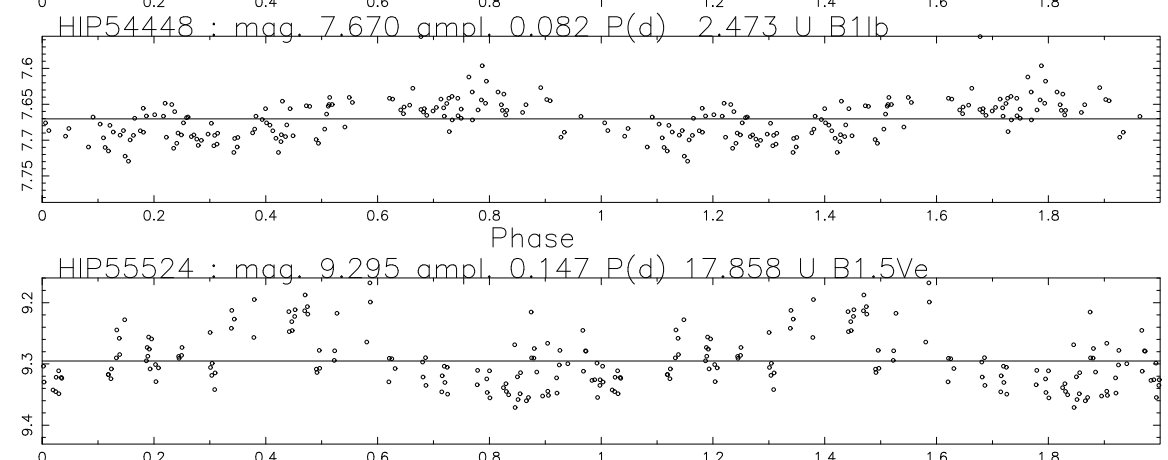

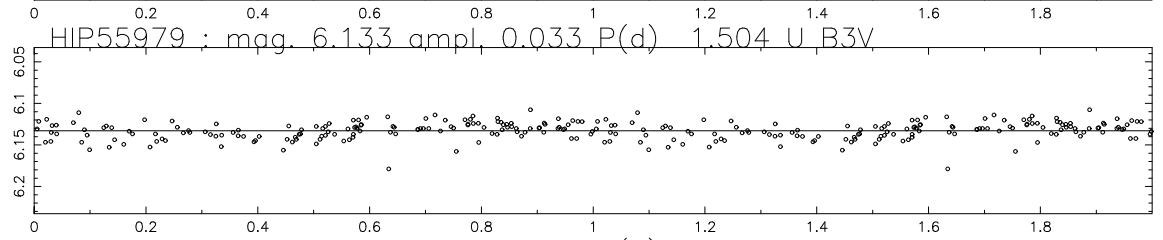
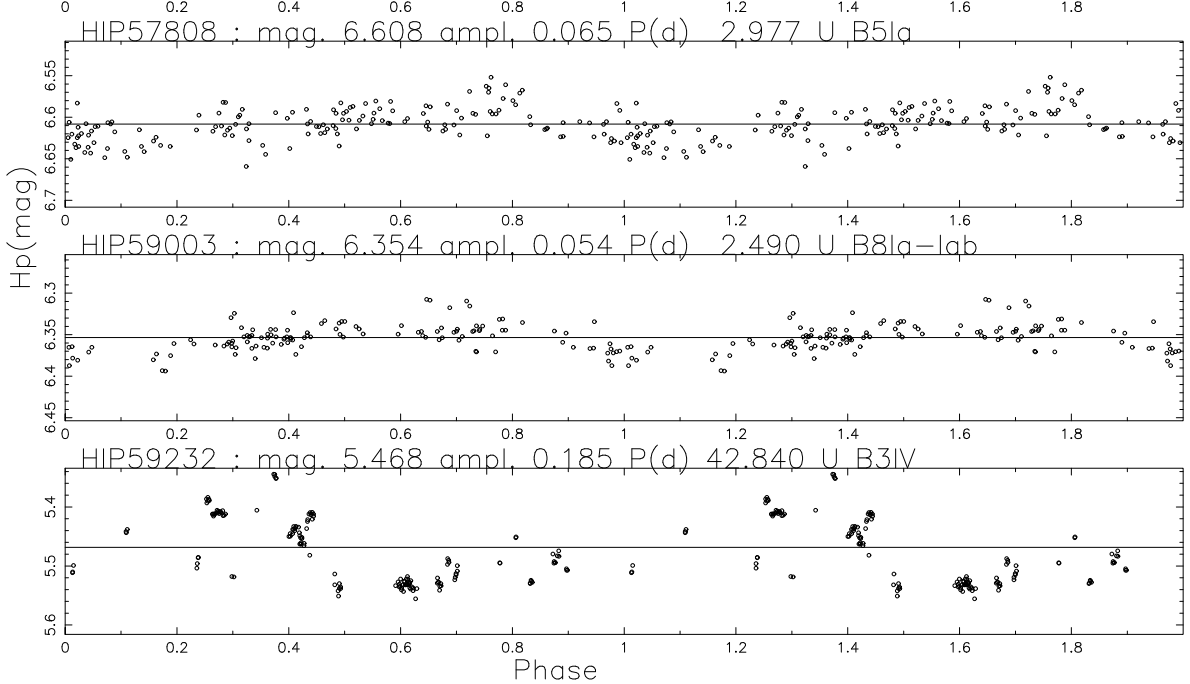


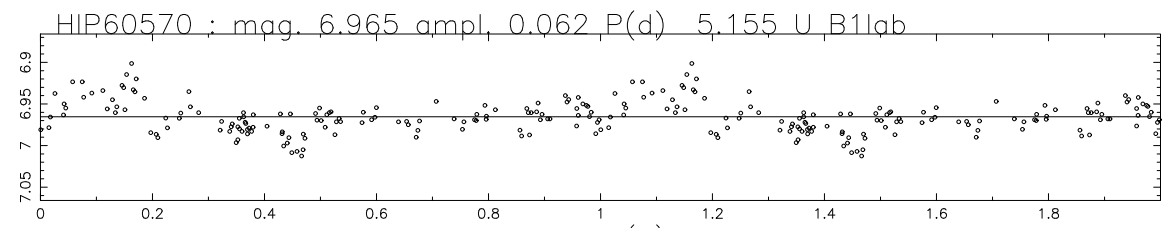

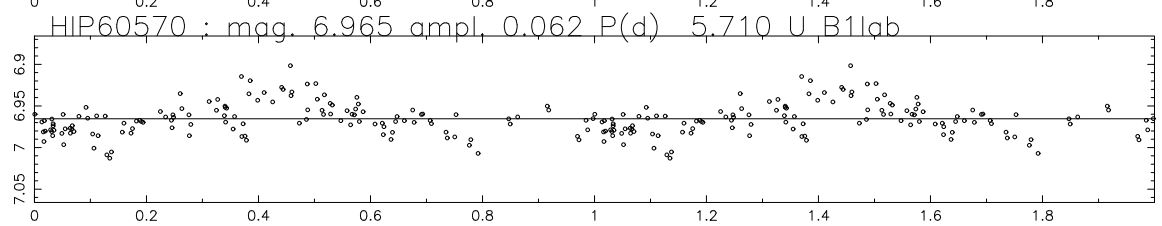

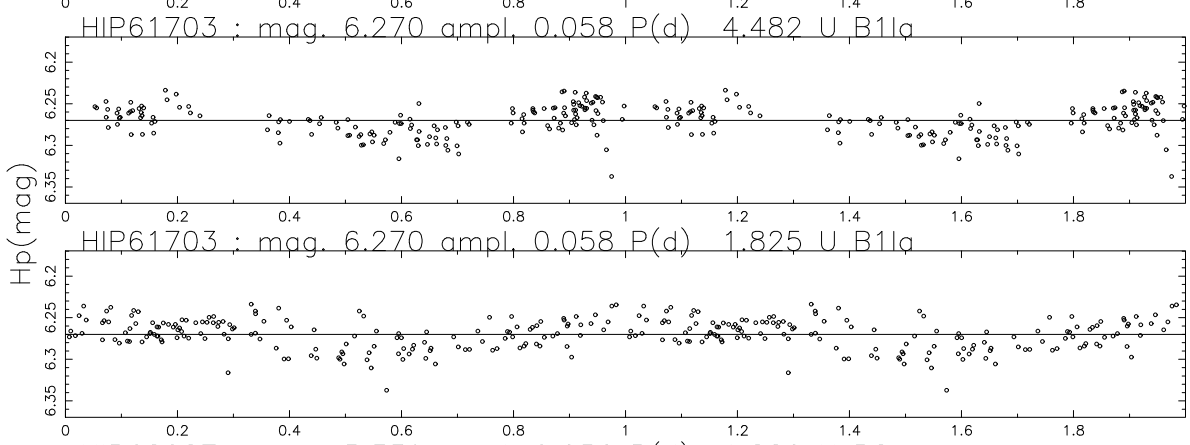

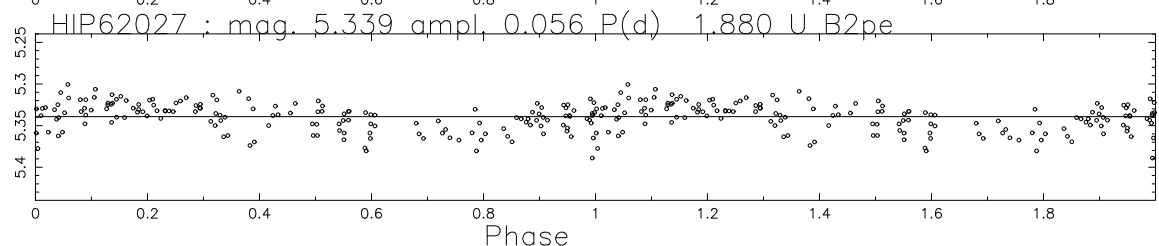

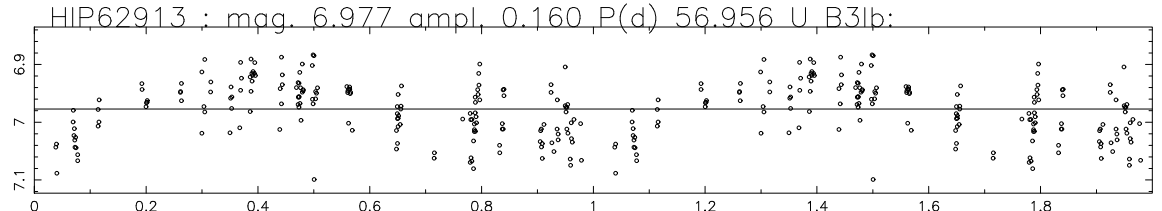

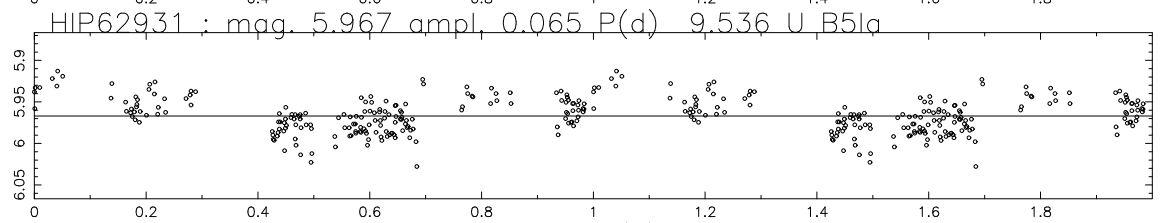

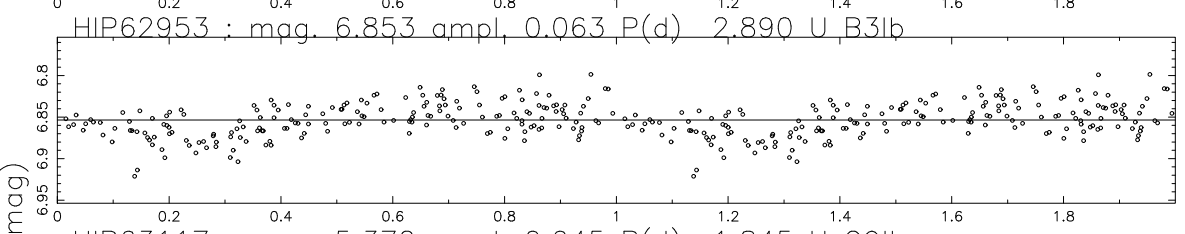

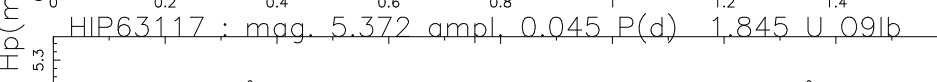

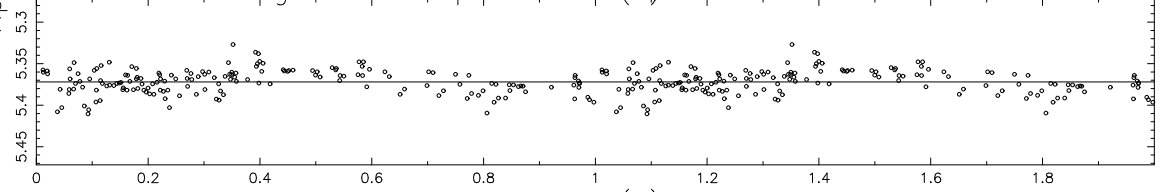

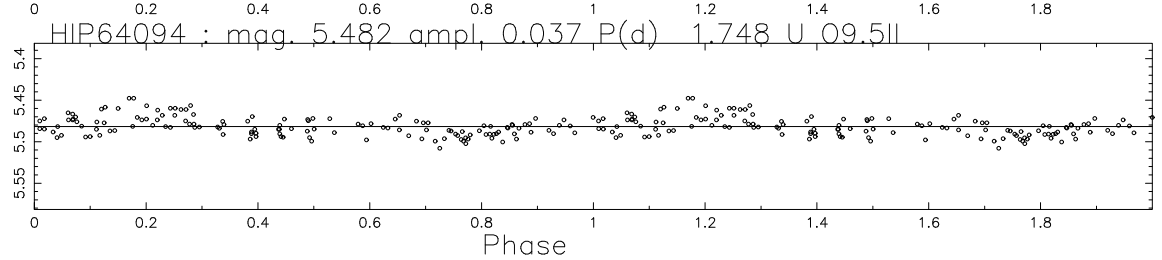



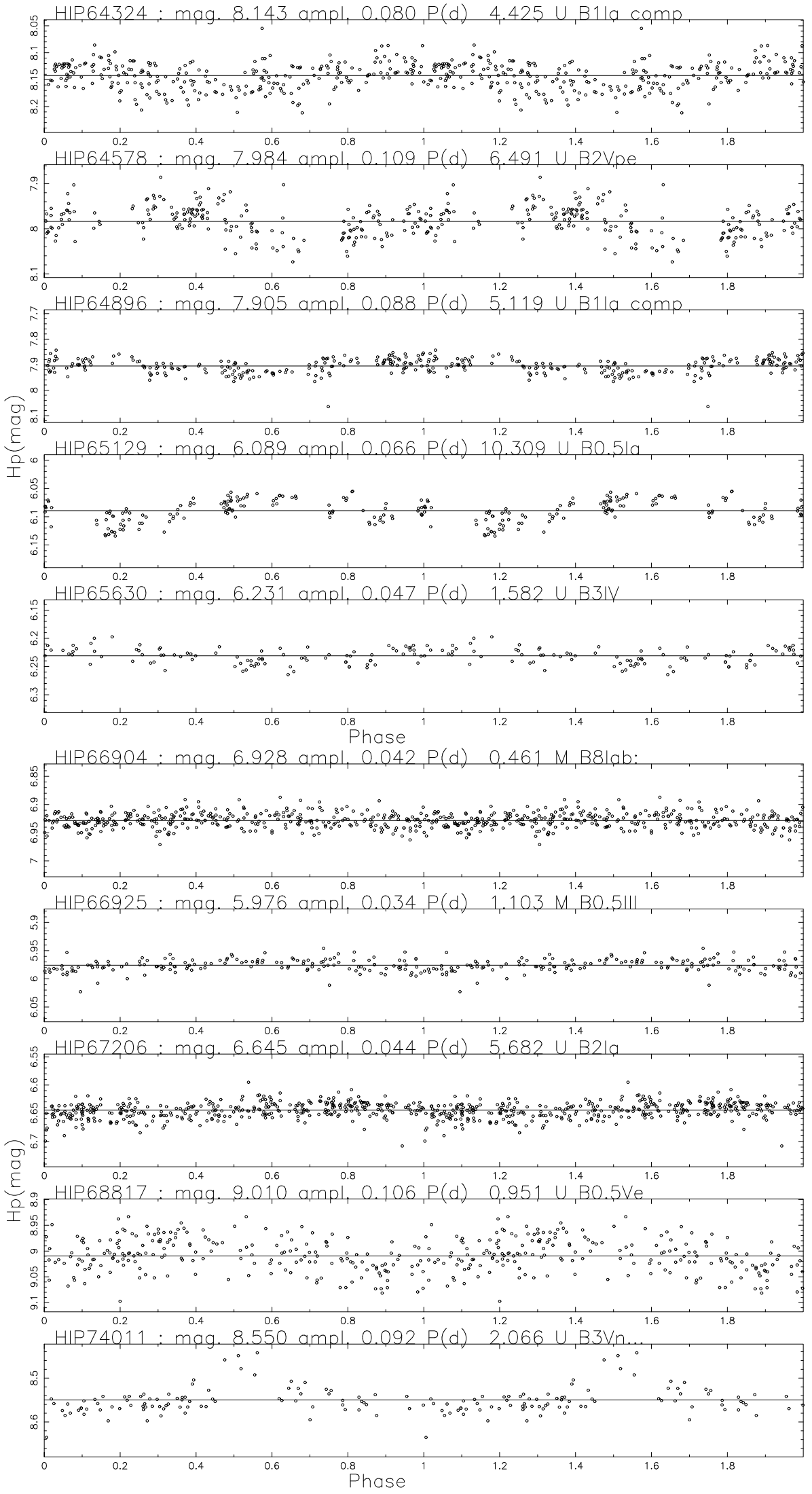

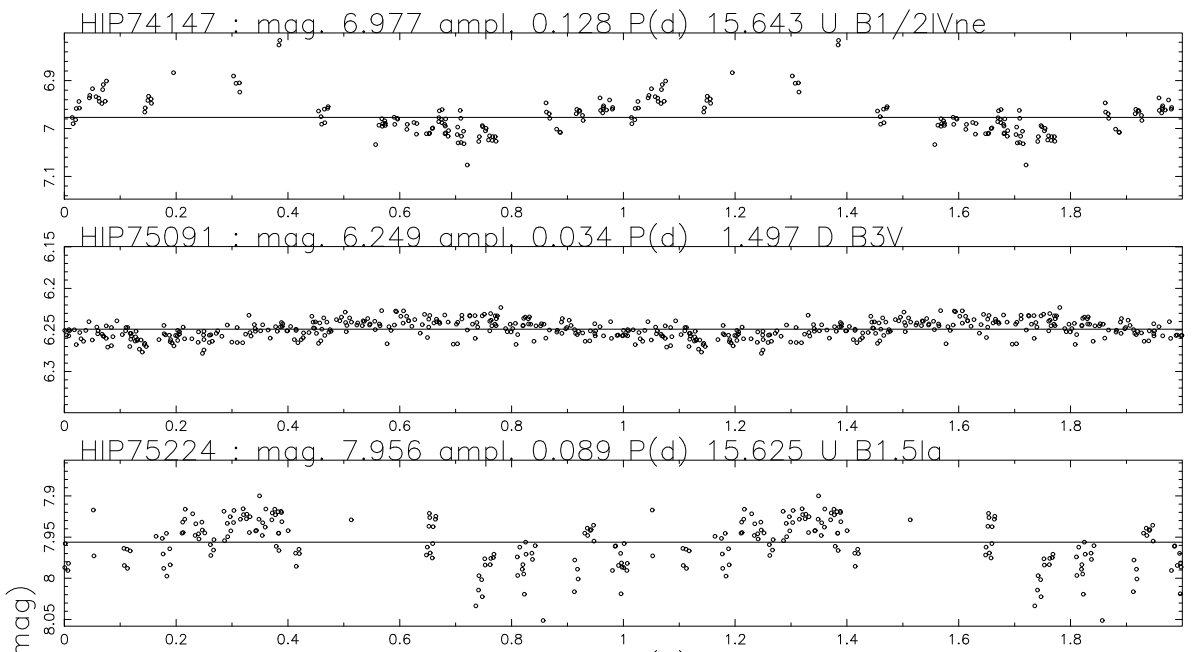

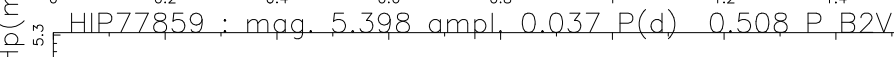
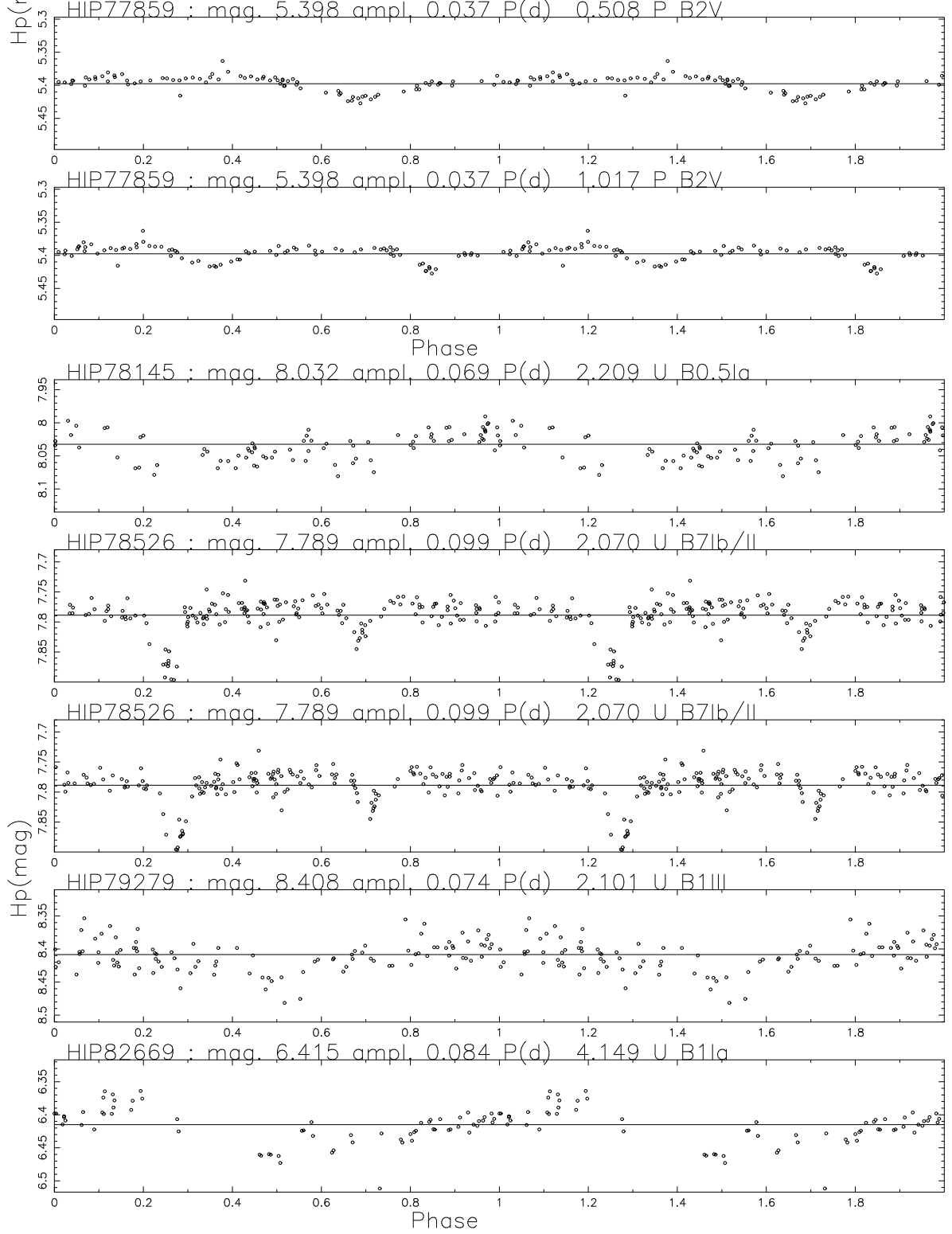

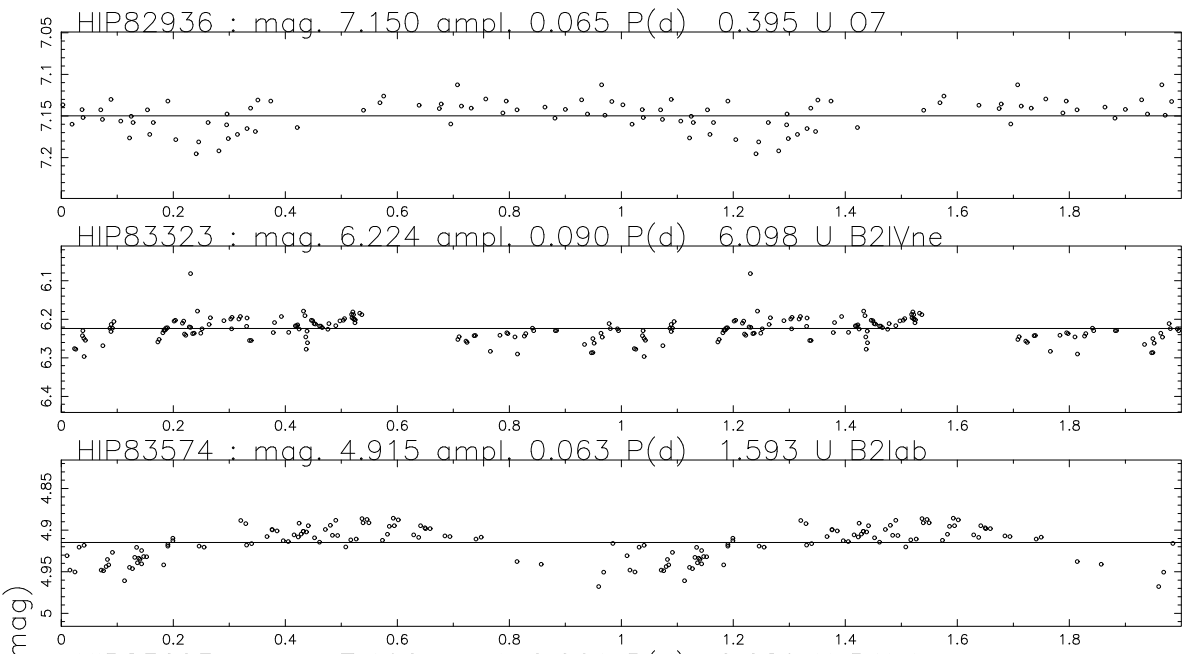

है- HIP,83603: mag. $7.196 \mathrm{ampl}, 0.061, \mathrm{P}(\mathrm{d}) \quad 0.089 \mathrm{U}^{1.4} \mathrm{~B} 1 \mathrm{lab}$ :
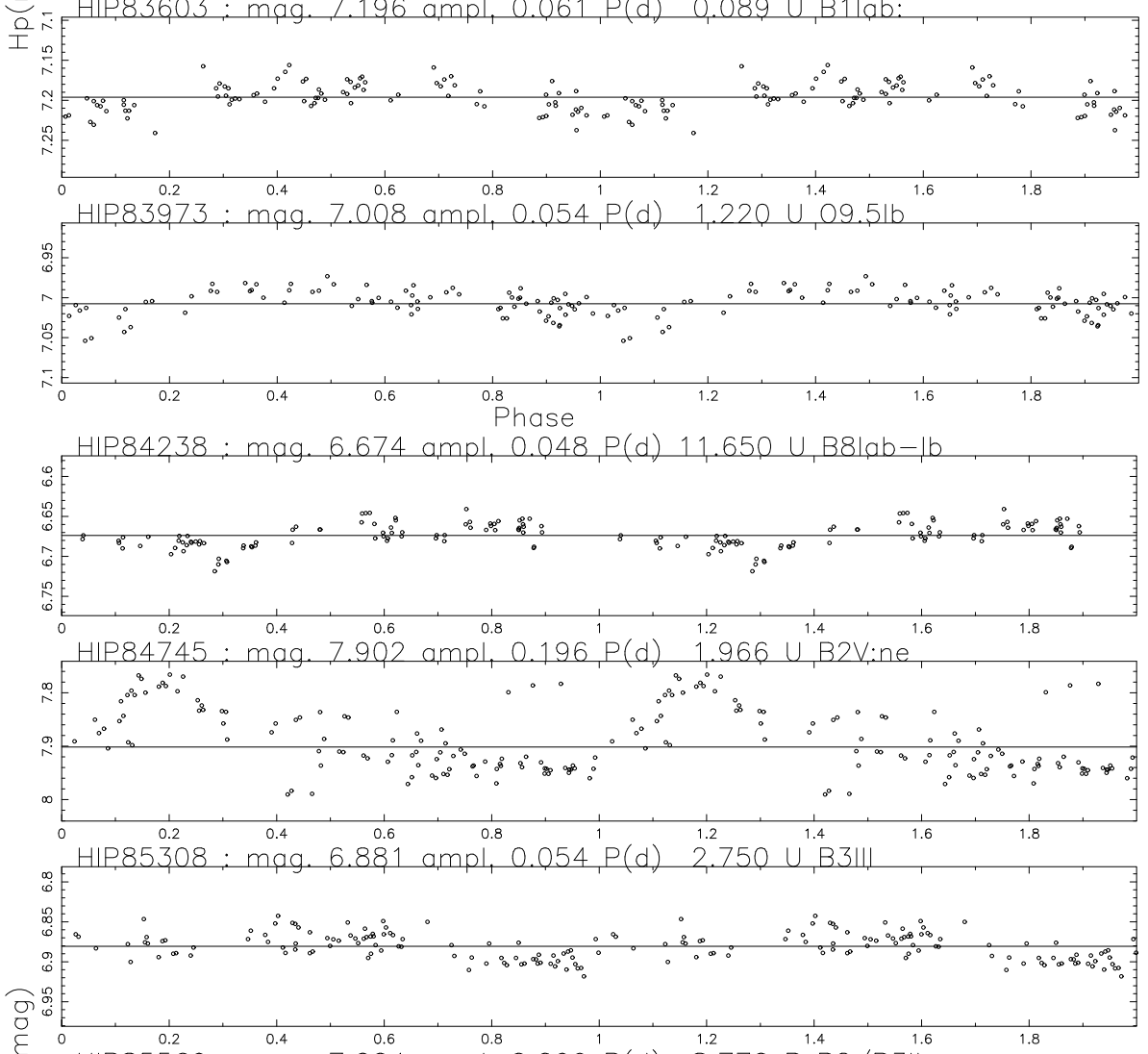

E HIP85569: mag. $7.004 \mathrm{ampl}, 0.099 \mathrm{P}(\mathrm{d}) \quad 8.770 \mathrm{P}, \mathrm{B} 2 / \mathrm{B} 3 \|_{1}$
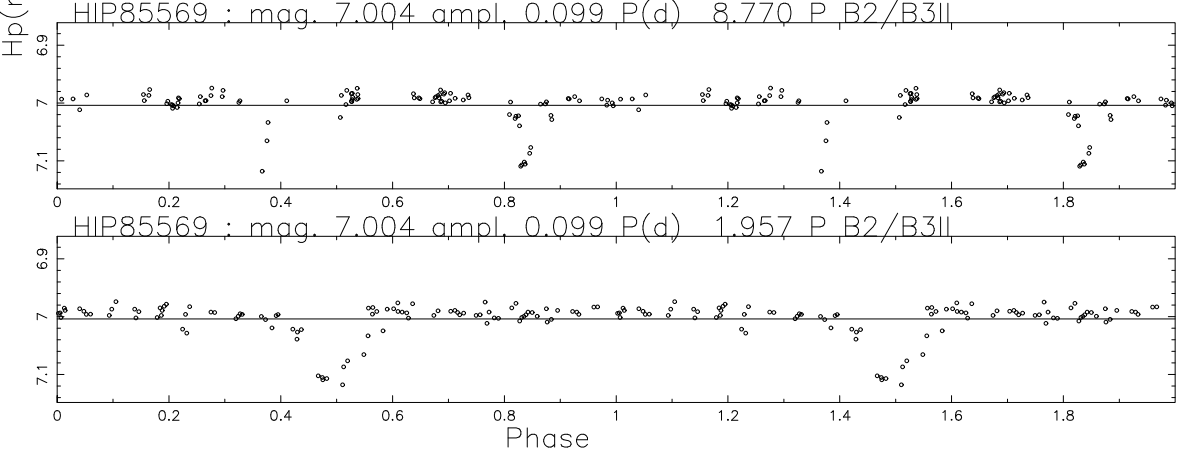

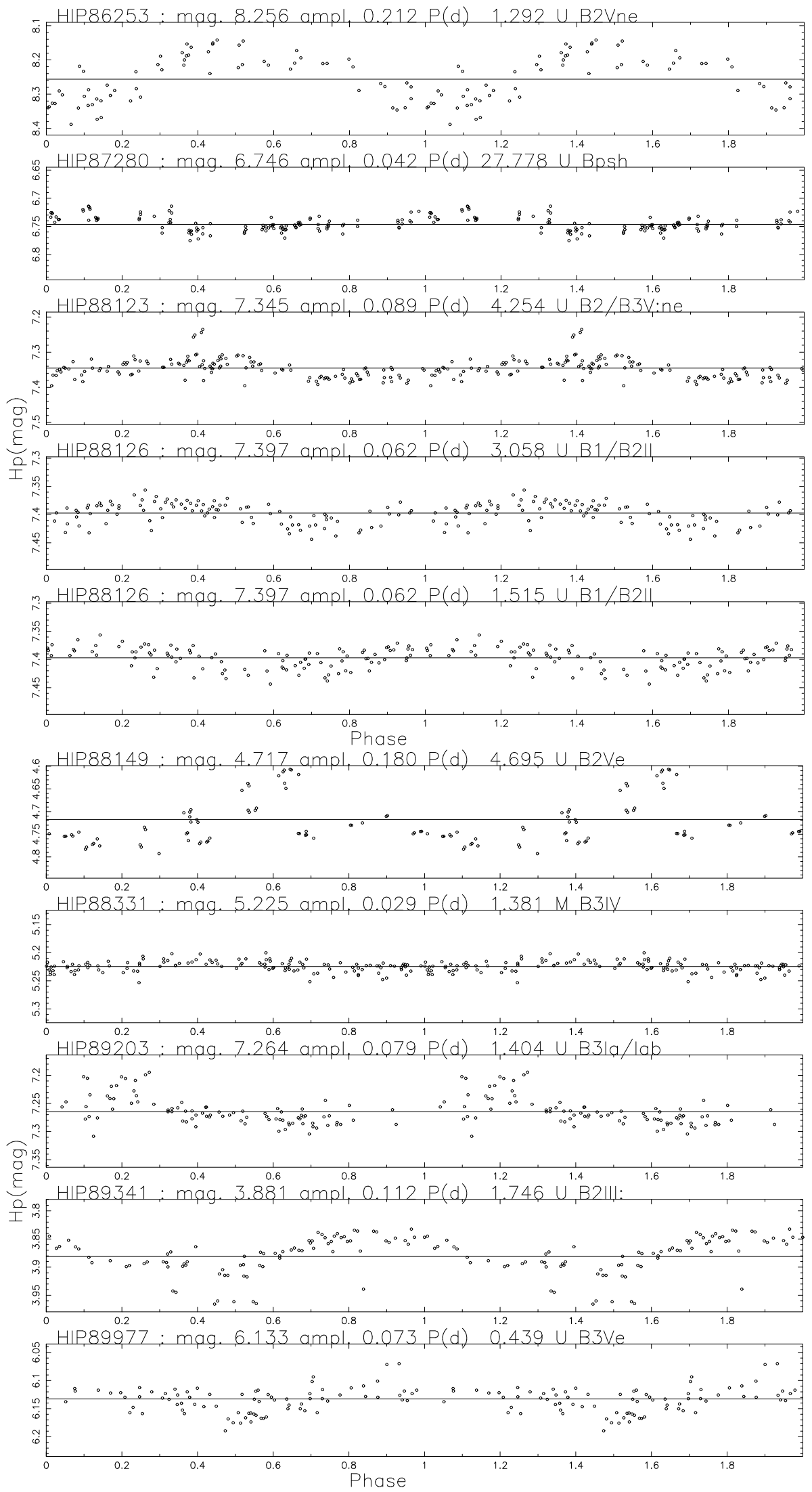

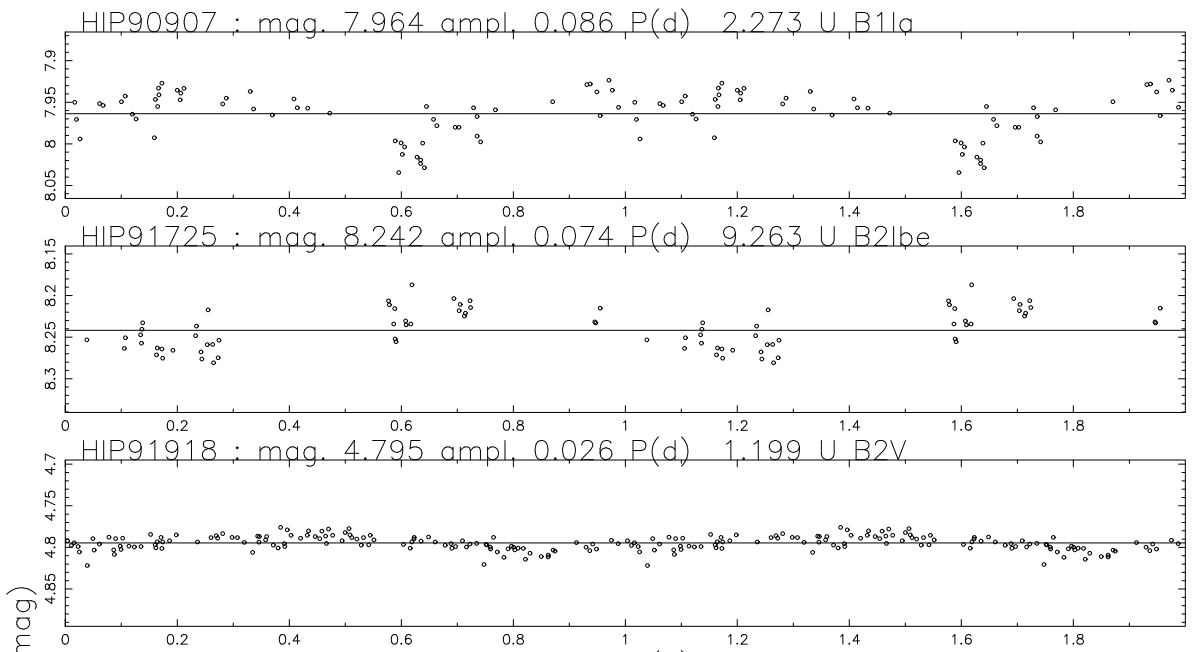

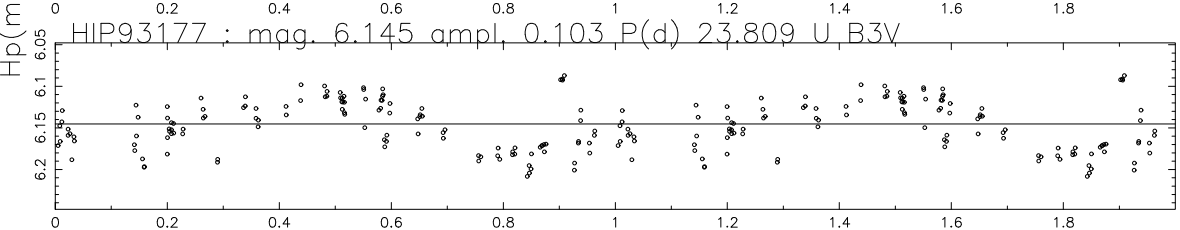

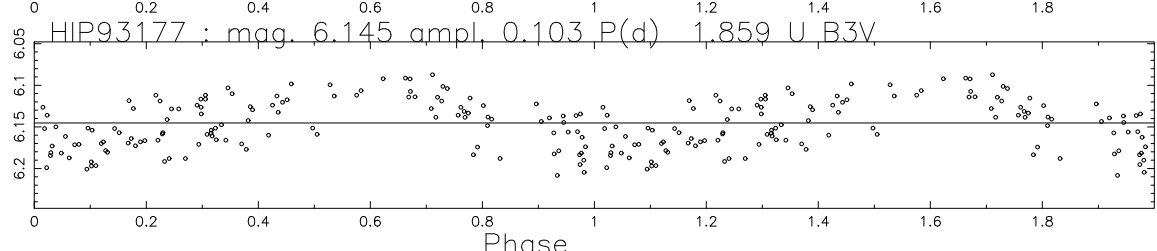

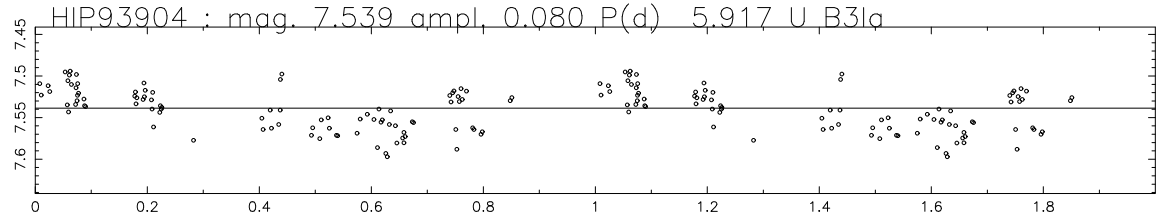

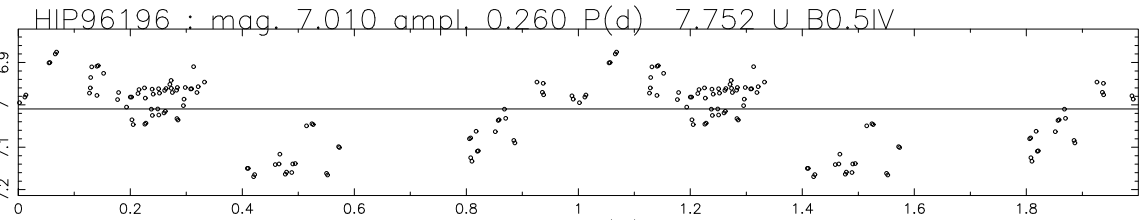

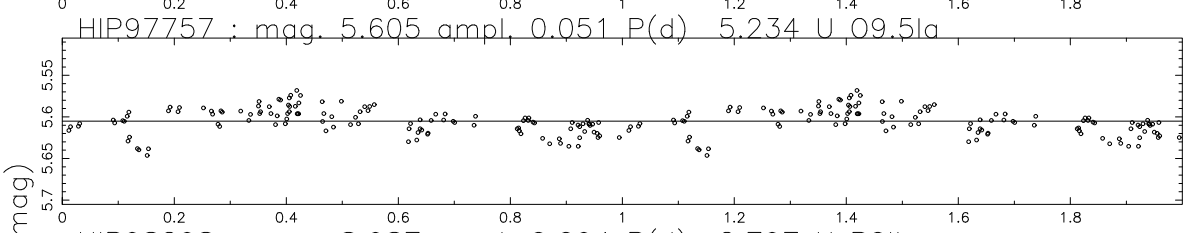

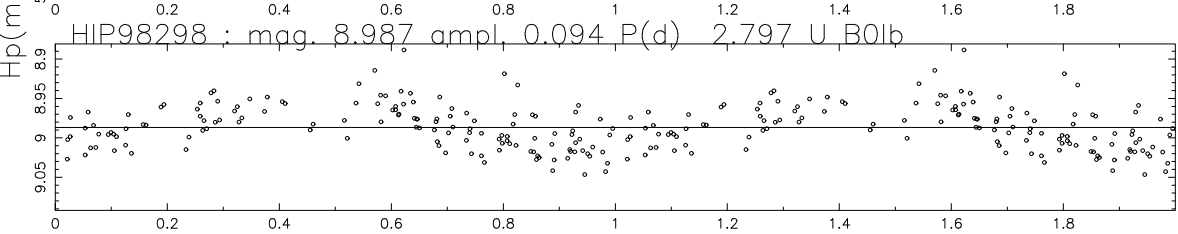

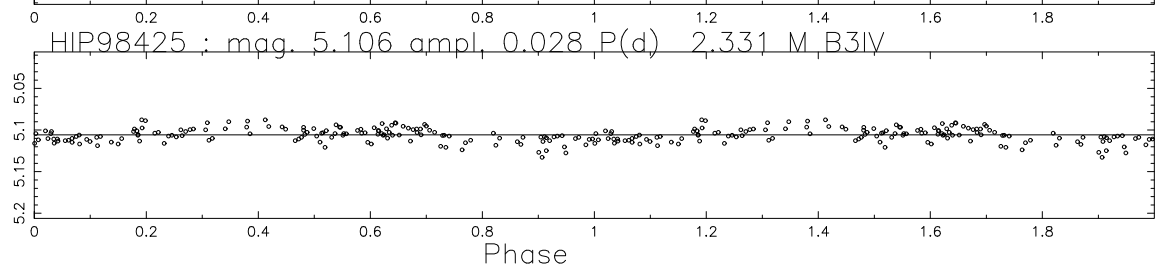



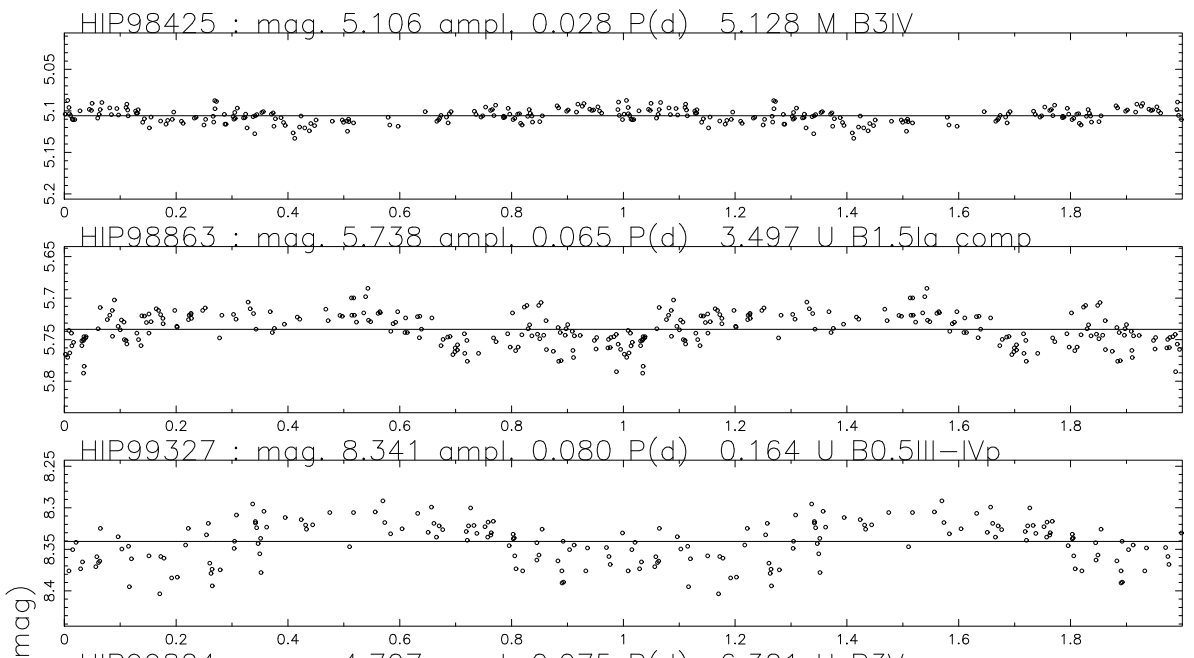

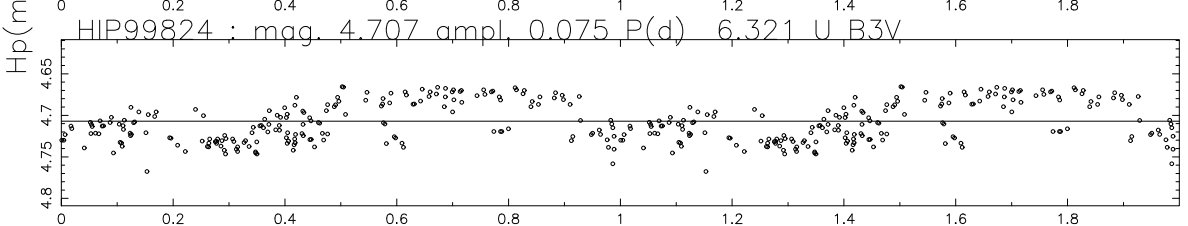
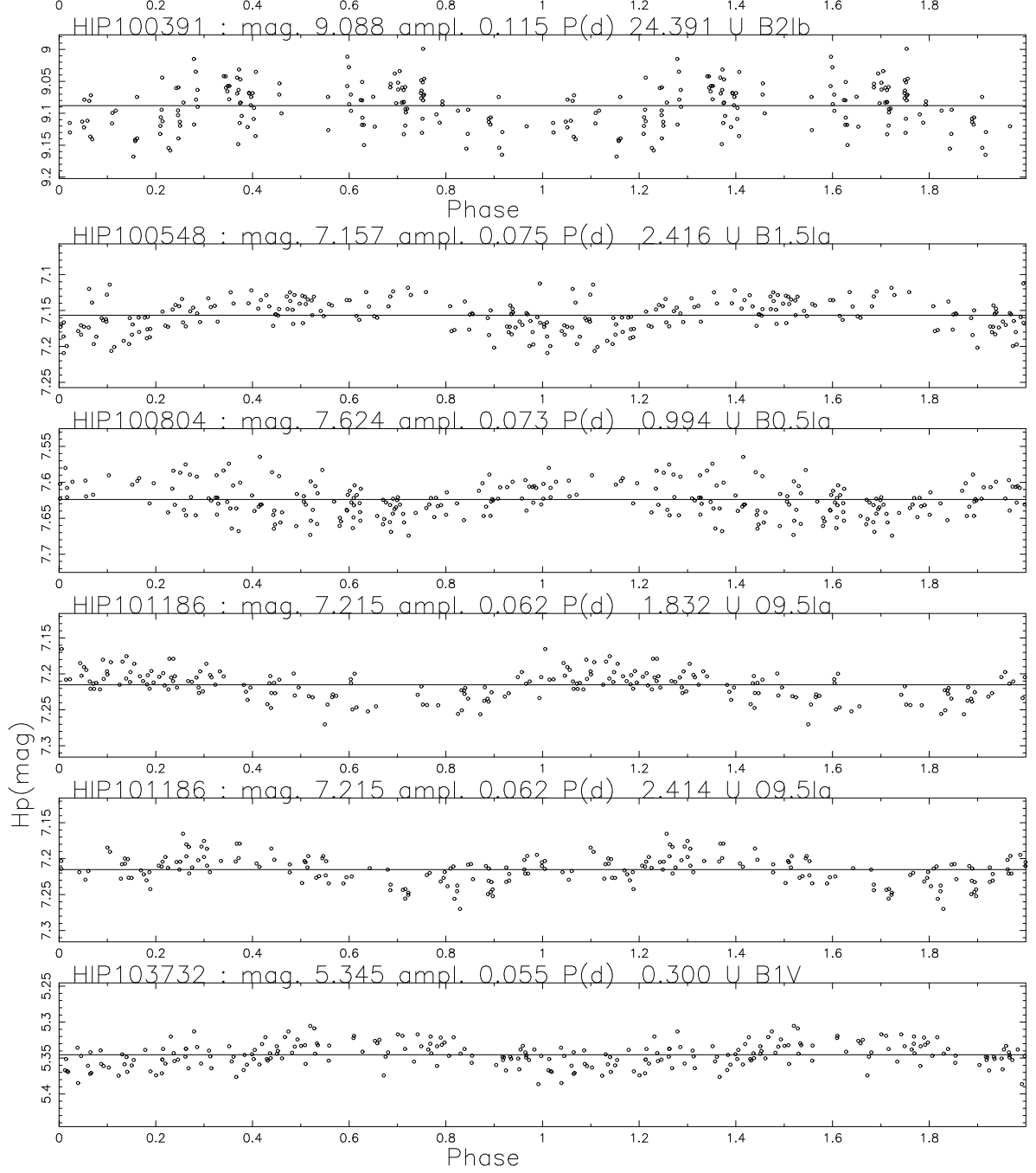

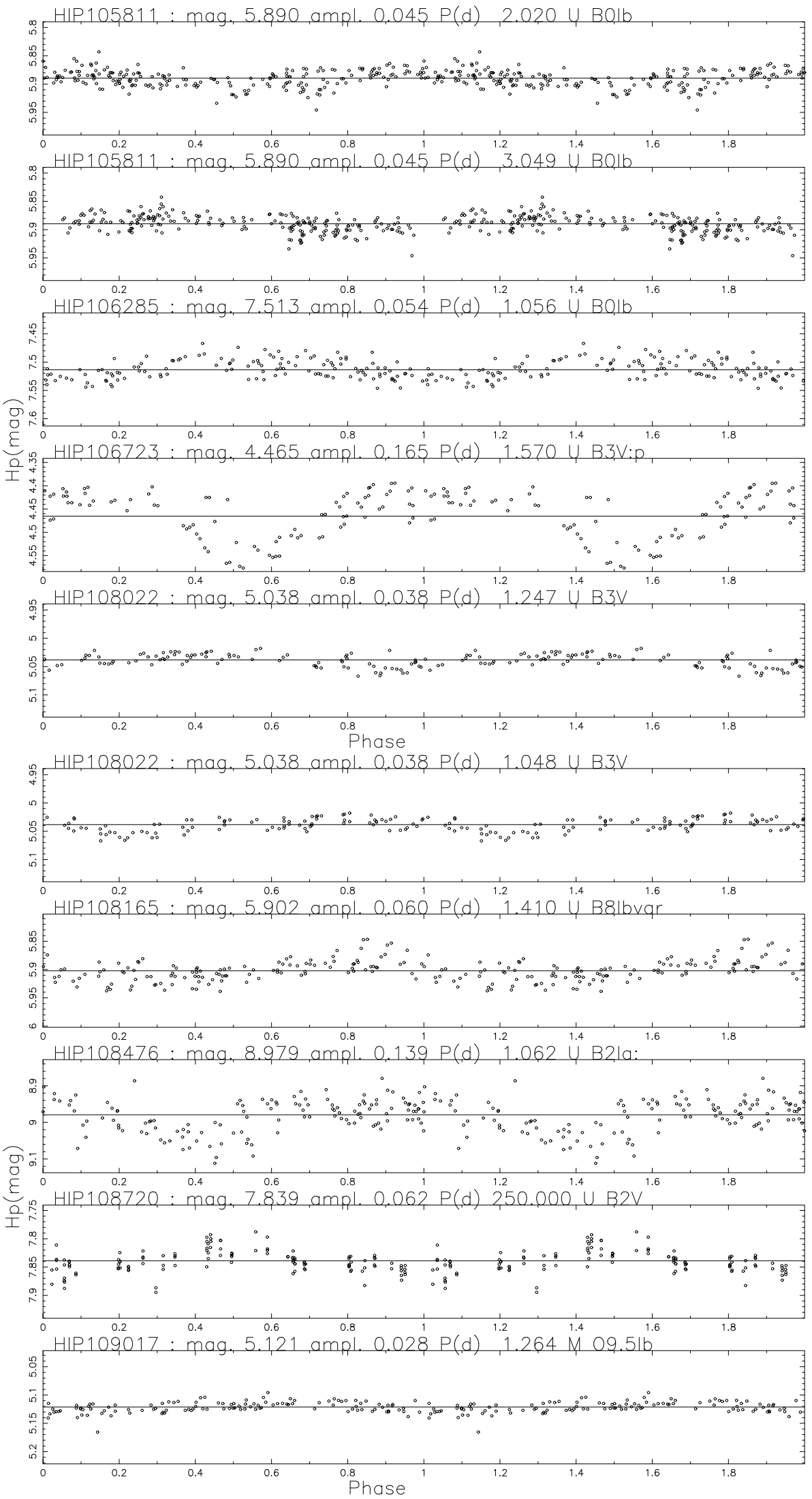

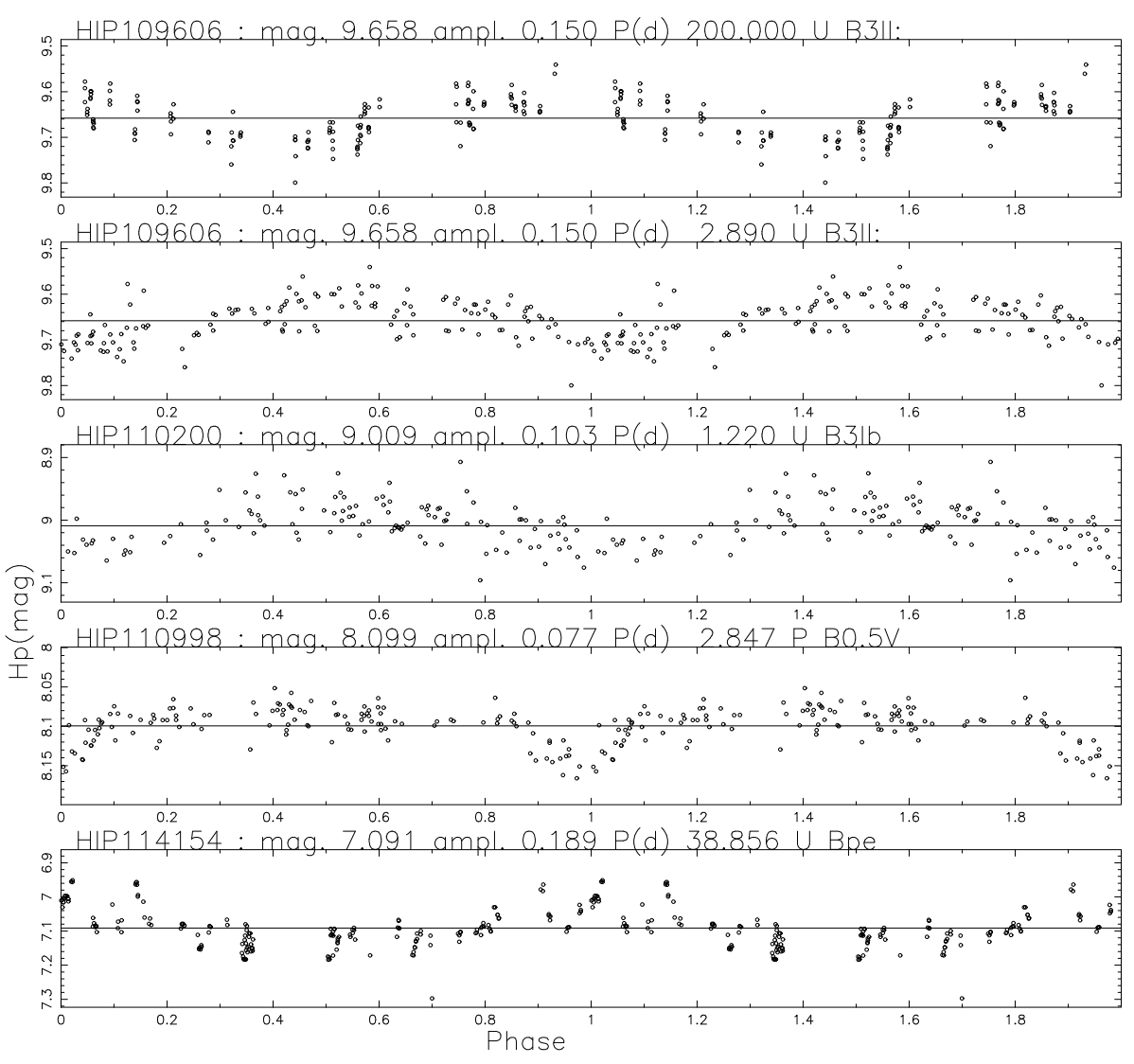


\section{B.3. Catalog of non-included periodic variable stars}
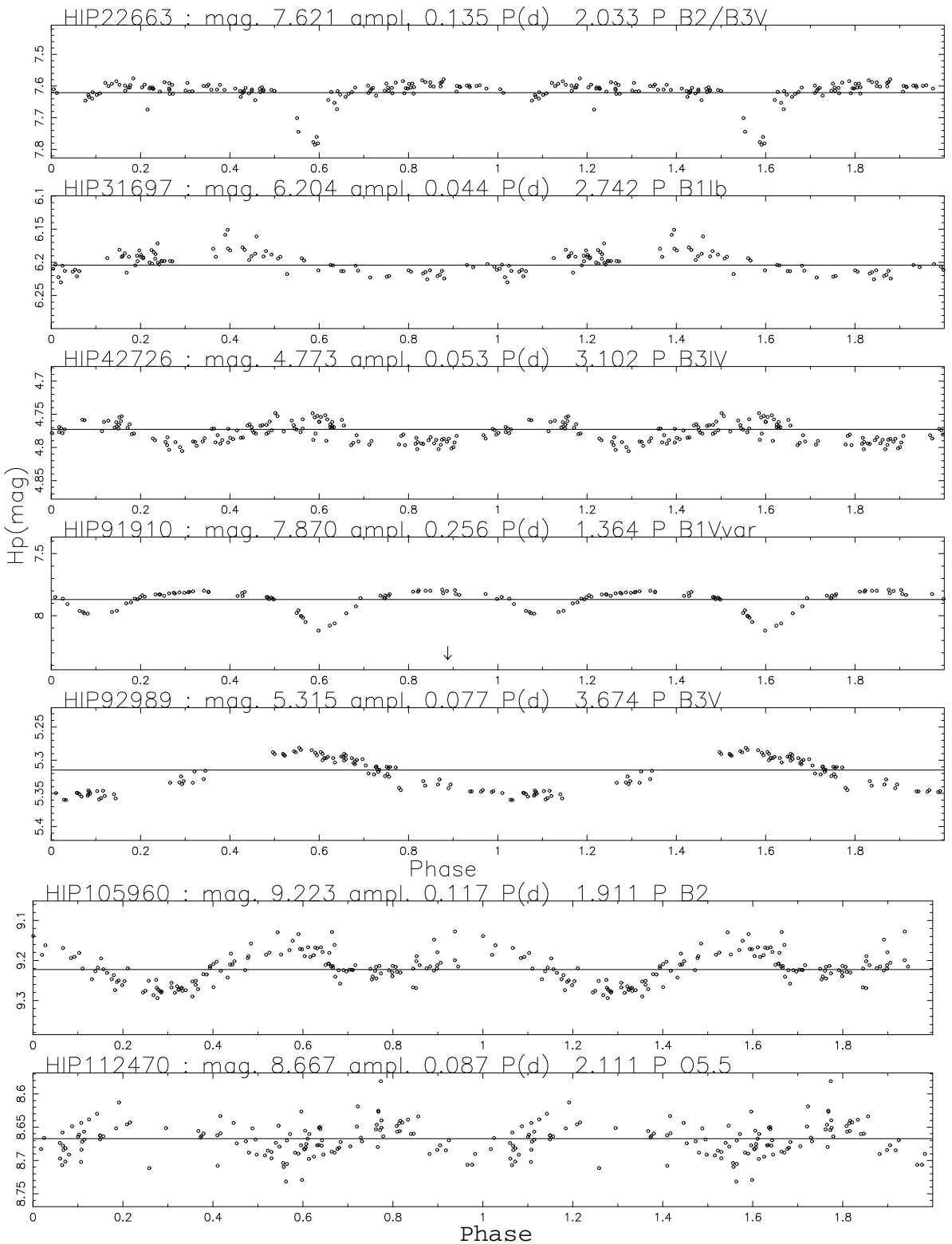

Fig. B.3. This figure plots $H p$ mag. vs. Phase. Epoch is arbitrarily JD-2 $440000=7800$, which is the beginning of the HIPPARCOS observations. The $H p$ magnitude, mean amplitude and periods are accurate to $\sim 0.1 \%$. 


\section{B.4. Catalog of periodic stars below $T_{\mathrm{h}}$}
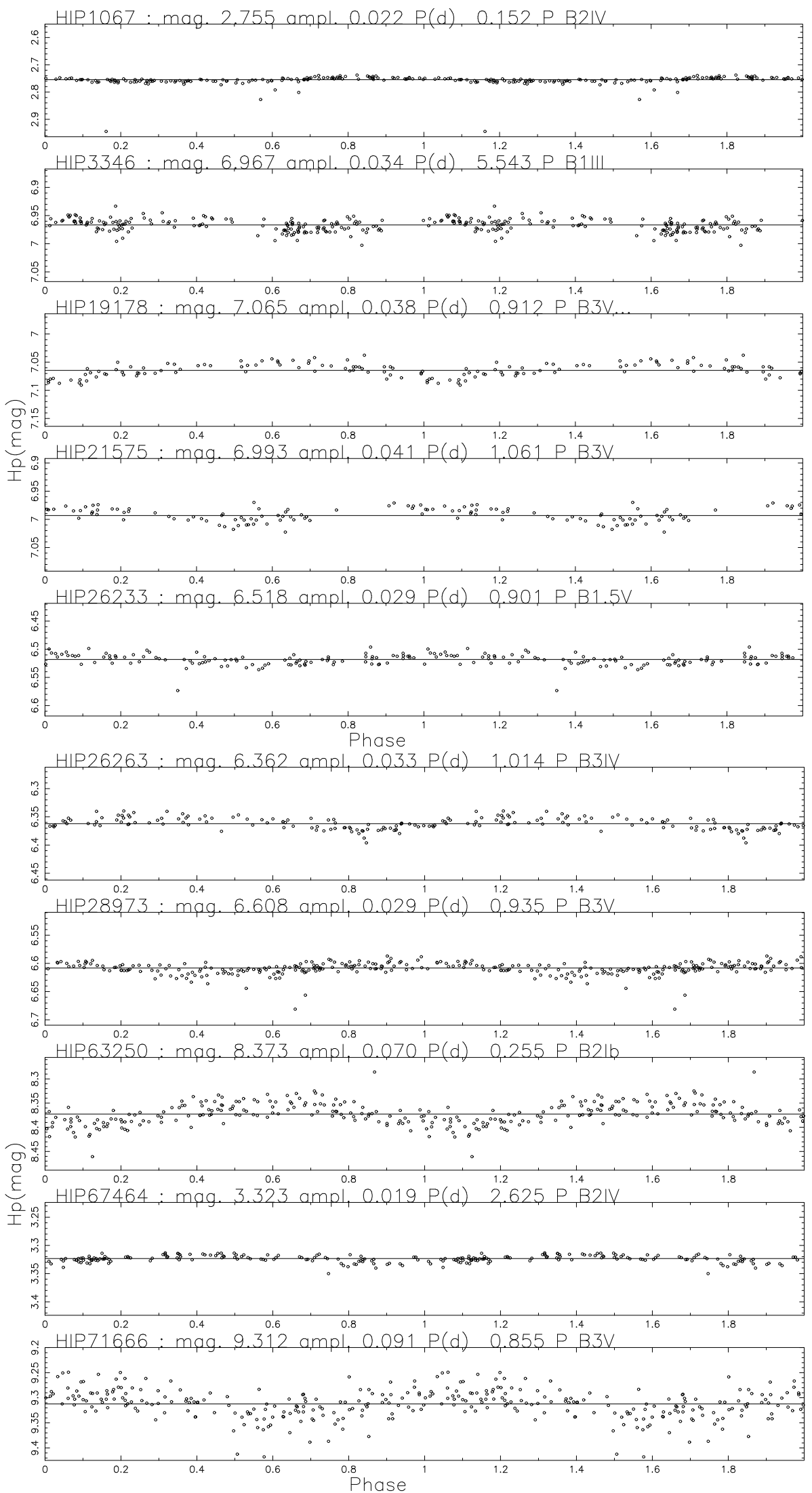

Fig. B.4. This figure plots $H p$ mag. vs. Phase. Epoch is arbitrarily JD-2 $440000=7800$, which is the beginning of the HIPPARCOS observations. The $H p$ magnitude, mean amplitude and periods are accurate to $\sim 0.1 \%$. 

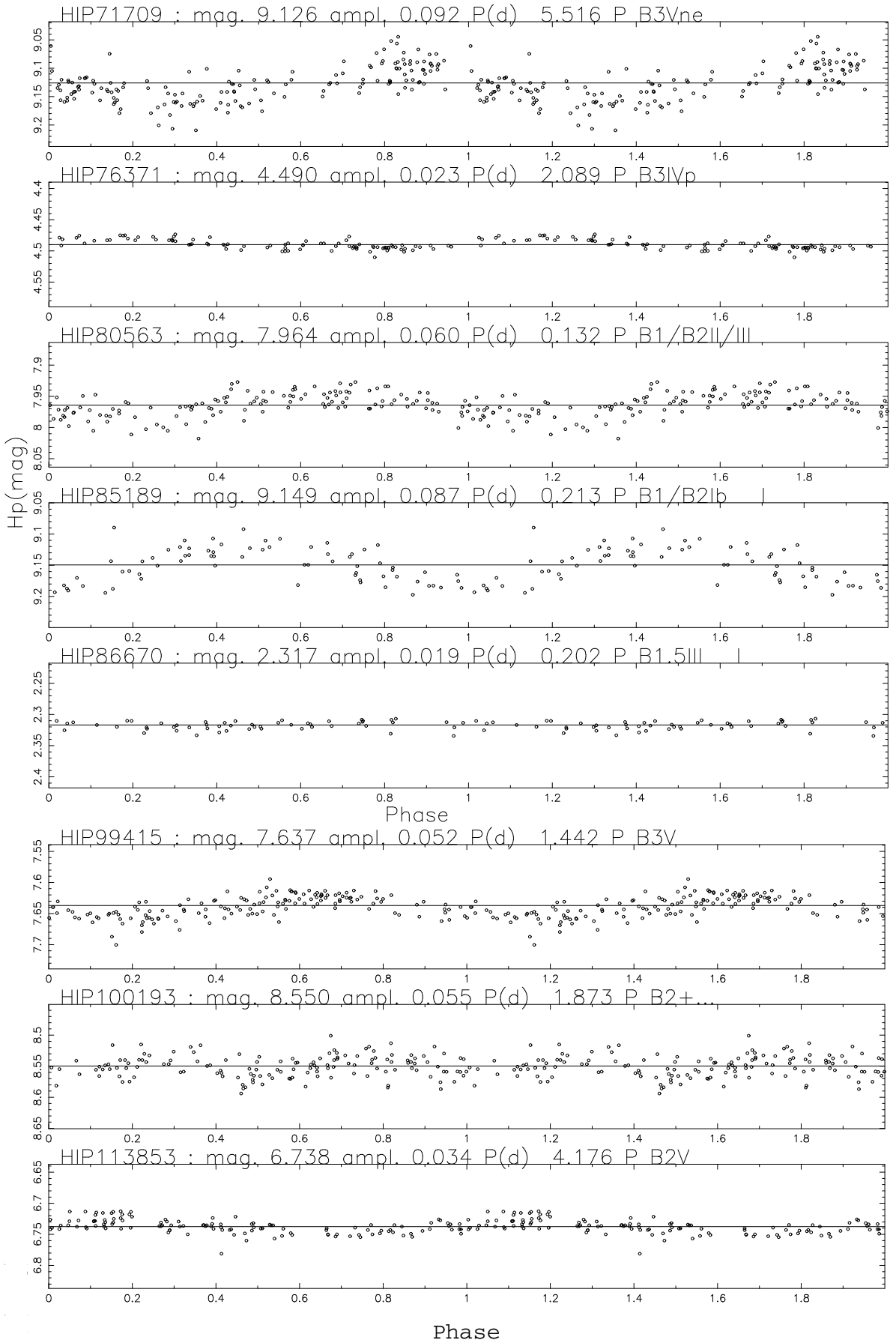